\title{
An Extensive Analysis of GNH Index
}

May 2012

Karma Ura

Sabina Alkire

Tshoki Zangmo

Karma Wangdi

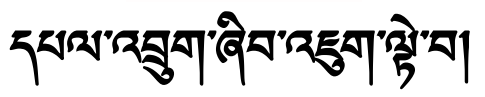

Centre for Bhutan Studies 


\title{
An extensive analysis of GNH Index
}

\author{
Copyright (C) The Centre for Bhutan Studies
}

First published 2012

ISBN 978-99936-14-67-8

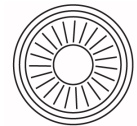 \\ The Centre for Bhutan Studies \\ Post Box No. 1111 \\ Thimphu, Bhutan \\ Phone: 975-2-321005, 321007 \\ Fax: 975-2-321001 \\ Email: cbs@druknet.bt \\ www.bhutanstudies.org.bt \\ www.grossnationalhappiness.com
}

The Centre for Bhutan Studies gratefully acknowledges the funding provided by International Development Research Centre (IDRC), Canada for data analysis and printing of the book. 


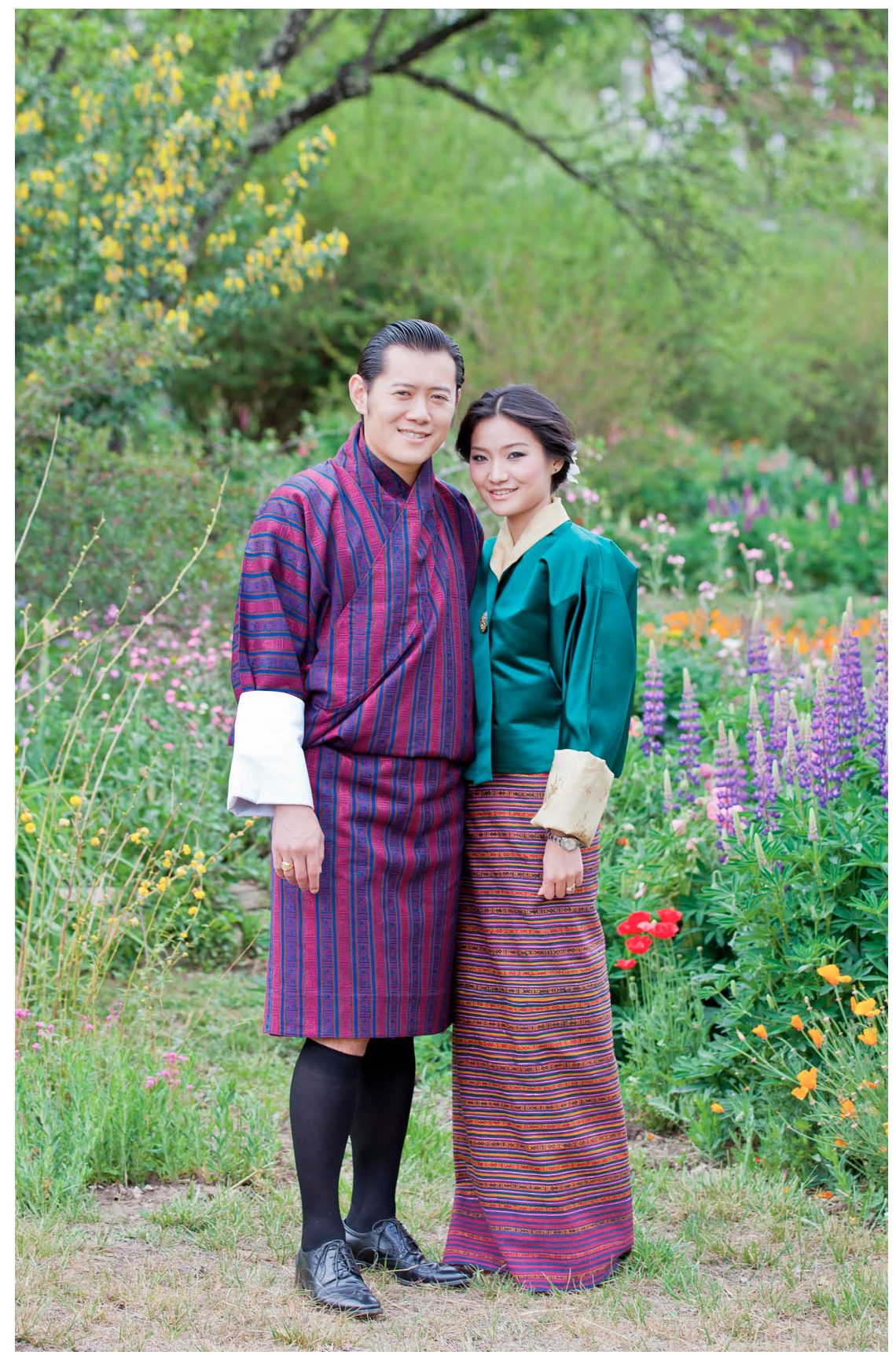

His Majesty the King Jigme Khesar Namgyel Wangchuck and Her Majesty the Queen Gyaltsuen Jetsun Pema Wangchuck 


\section{Acknowledgements}

We are grateful to Mr Dorji Penjore, Chief Research Officer at CBS for coordinating the conduct of the survey. Our sincere gratitude are owe to Kuenzang Lhadon, Sangay Chophel, Sangay Thinley, Thinley Jamtsho, and Dendup Chophel (Researchers at CBS) and the 109 enumerators, for spending long hours each day interviewing respondents. The support staffs at CBS equally deserve our appreciation for painstakingly entering the monumental amount of data collected over the course of many months. We are also grateful for the support extended by Paola Ballon Fernandez, Gisela Robles Aguilar, and Mauricio Apablaza Salinas of OPHI for assisting with data analysis.

Furthermore we remain indebted to Dzongdags, Drungpas, Gups, Tshogpas, Chuepoens and other officials of the 20 Dzongkhags for their valuable support in conducting this survey. Most importantly, we would like to offer our deep gratitude to all the respondents, without whose time and cooperation, this report can never come into being. Their time and warm hospitality coupled with the understanding of their family members to let respondents be interviewed by letting them off even from the agricultural field made this extraordinarily long interviews possible.

Finally we would like to thank United Nations Development Programme (UNDP, Bhutan), Joint Support Programme (JSP) supported by DANIDA and UNDP-UNEP, and Royal Government of Bhutan (RGoB) for financing the survey and International Development Research Centre (IDRC), Canada for financing the data analysis and printing of this book. 


\section{Contents}

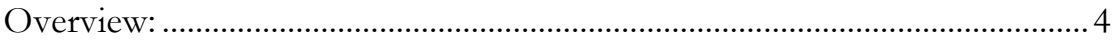

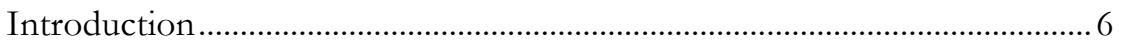

History of GNH as a Policy Priority ……………………………………...... 6

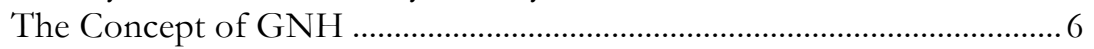

Pillars and Dimensions of GNH .................................................................. 9

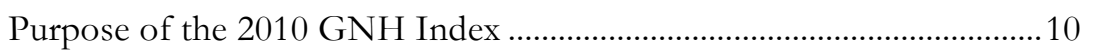

Part I: Construction of the 2010 GNH Index...............................................13

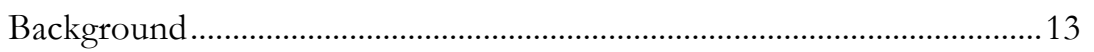

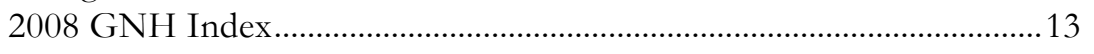

2010 Gross National Happiness Survey.................................................... 14

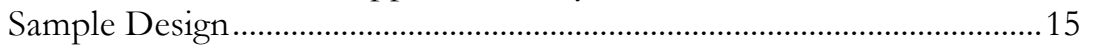

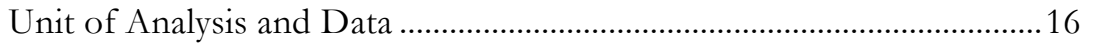

Choice of GNH indicators: 13 trials..........................................................16

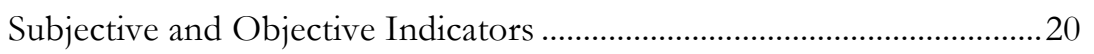

Domains and Indicators ..............................................................................22

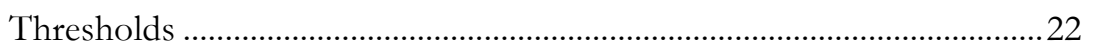

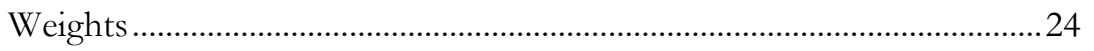

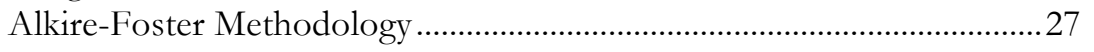

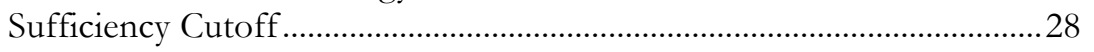

Happiness Gradient................................................................................29

Identification for the GNH Index .............................................................. 30

Sufficiency and Insufficiency among the Not-Yet-Happy .........................32

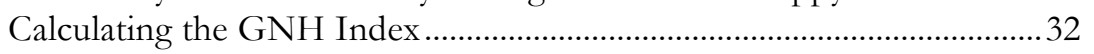

Methodology: GNH Index............................................................................33

The GNH Index: Formulae and Interpretation.............................................35

Two Analyses: Understanding Happiness and Increasing Happiness.... 37

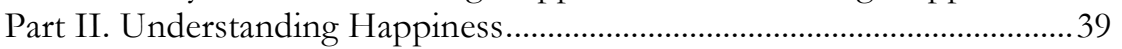

Results across the Happiness Gradient ..........................................................4 40

Domain Composition of GNH ................................................................ 42

Happiness by Dzongkhag......................................................................... 44

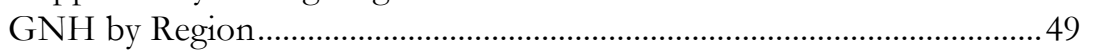

GNH by Gender...................................................................................... 49

GNH by Age Group and Marital Status ..................................................... 52

GNH by Educational Level and Occupational Status ...............................52

Part III. GNH Index and Policy: Increasing Happiness ...............................53

Achievements of Happy vs Not-yet-happy People ......................................55

Structure of the Insufficiencies among the not-yet-happy .........................56

Insufficiencies by dzongkhag and rural-urban regions ...............................58

Insufficiencies by Gender......................................................................... 59 
Insufficiencies by Age and Marital Status .....................................................59

Insufficiencies by Occupational Category …………………………………....61

The Deeply Happy ………………………………………………….....6 62

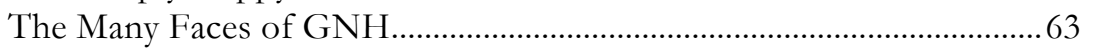

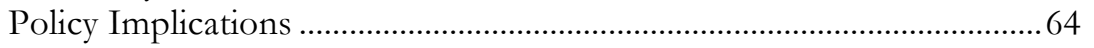

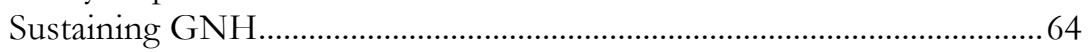

Robustness Analysis - Indicators, Cutoffs, k, Weights. ..............................65

Robustness Ia: Standard Errors ..................................................................66

Robustness Ib: Robustness to Changes in the Happiness (k) Cutoff.....67

Robustness II: Robustness of Dzongkhags' Contributions to Overall

Happiness by Cutoff and Weights ............................................................... 70

Robustness III: Robustness of Indicators' Contributions to Overall

Happiness by Cutoff and Weighting Scheme.............................................75

The Way Forward: GNH as a Policy Tool …………………………........... 82

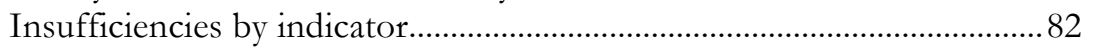

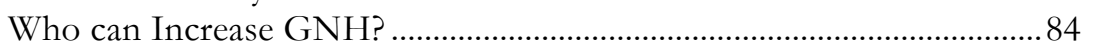

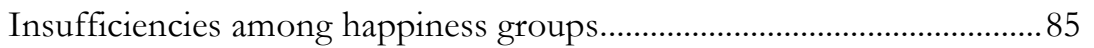

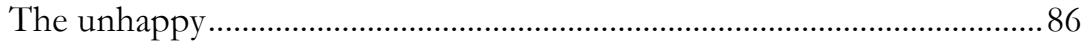

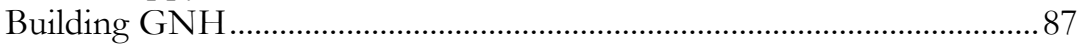

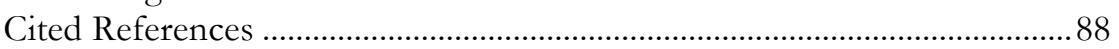

Appendix 1. Sampling and weighting ............................................................114

Generation of Sample Weights.................................................................114

Appendix 2. Subjective Questions ...................................................................116

Appendix 3. Self-Report Questions .............................................................118

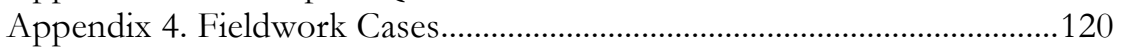

1. Psychological Wellbeing ...........................................................................123

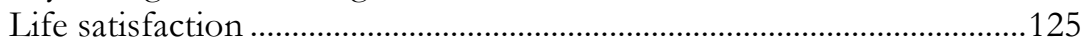

Emotional Balance (Positive and Negative Emotions)............................128

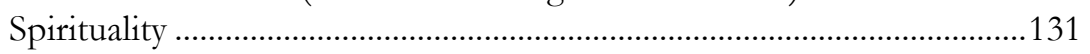

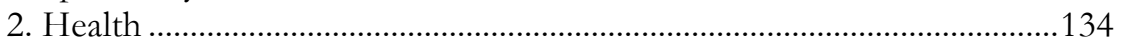

Self-reported health status .........................................................................135

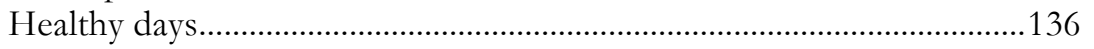

Long-term disability ................................................................................137

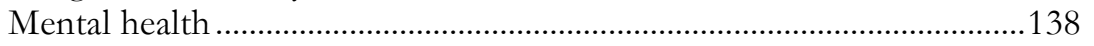

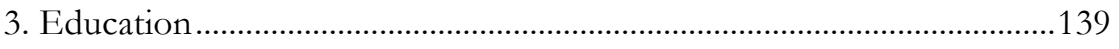

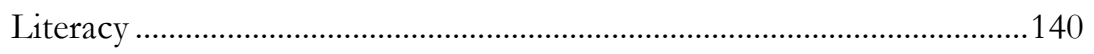

Educational qualification ........................................................................... 140

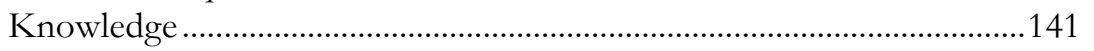

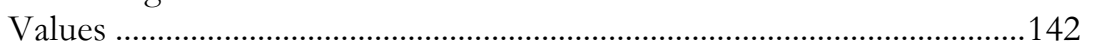

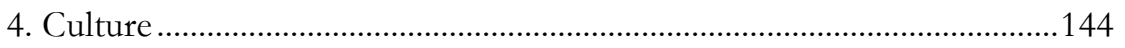

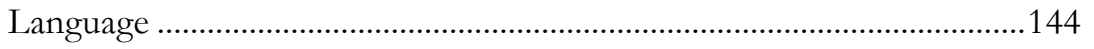

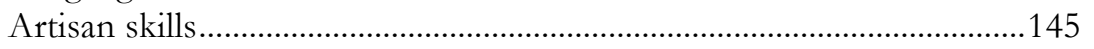

Socio-cultural participation ....................................................................146

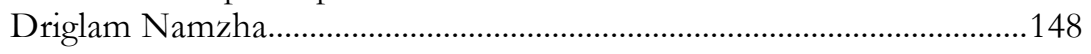




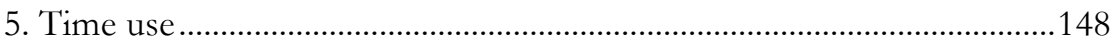

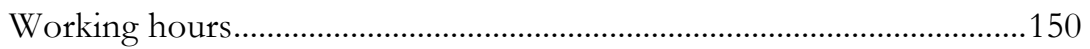

Sleeping hours ……………………………………………………....154

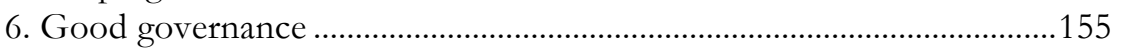

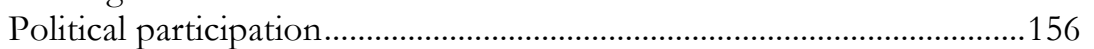

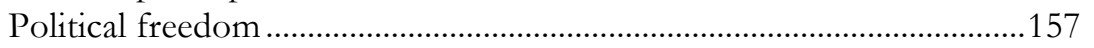

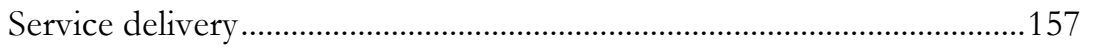

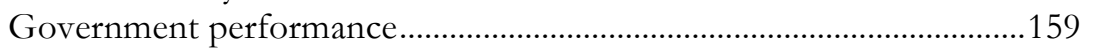

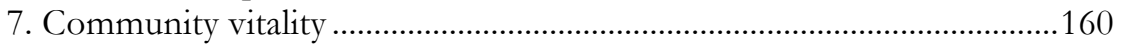

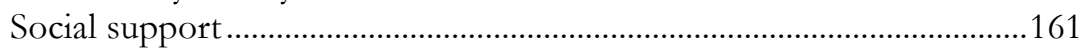

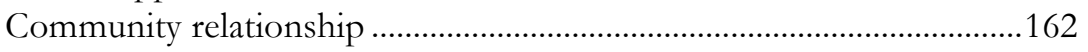

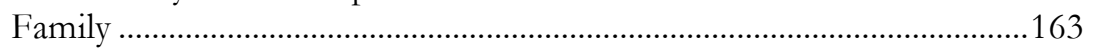

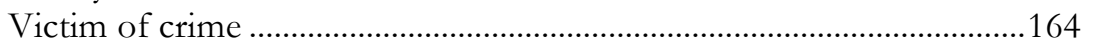

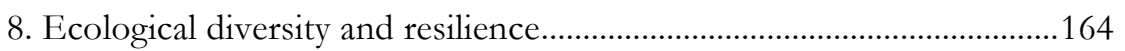

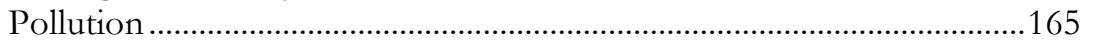

Environmental responsibility ................................................................166

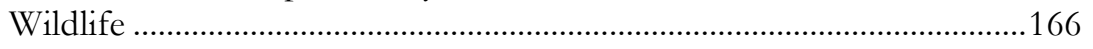

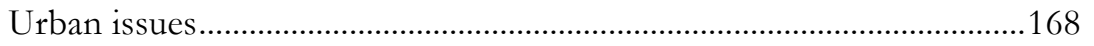

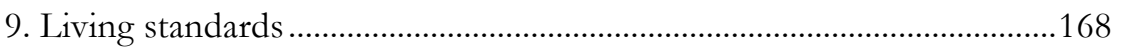

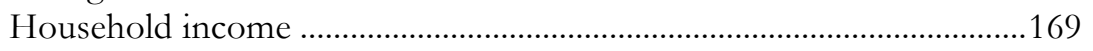

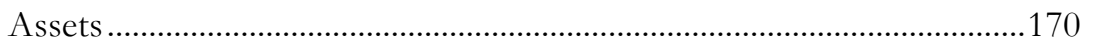

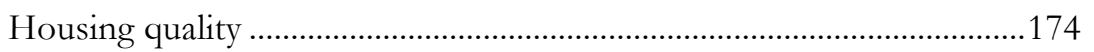

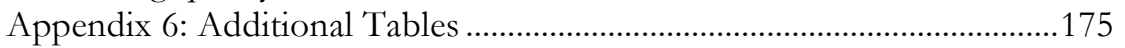




\section{Overview:}

Multidimensional measures of the quality of life and wellbeing are increasingly discussed. Many wonder whether a multidimensional measure of wellbeing might provide a policy-relevant and relatively accurate overview of such a complex phenomenon, without losing important details. This paper presents and analyses the 2010 Gross National Happiness (GNH) Index was developed at the Centre for Bhutan Studies for the Royal Government of Bhutan. The new GNH Index provides a summary statistic of the wellbeing of individuals in nine domains, which are instrumented by 33 indicators and draw on 124 variables. It is constructed using an adaptation of the Alkire-Foster methodology for poverty measurement, in which a first set of indicator cutoffs reflect sufficiency - how much is 'enough' - rather than poverty. The second (cross-indicator) cutoffs categorise the population into four levels of GNH, creating a 'happiness gradient'. The data come from a nationally representative multi-topic survey that is representative by district and region, and the GNH Index and associated statistics can be used to show the joint distribution of achievements each respondent enjoys, as well as any insufficiencies she experiences. Our assessment based on a series of robustness tests included here is that this index's methodology and results are rigorous and that they can be used to generate policy-relevant insights and analyses. As the field of multidimensional measurement of well-being is entering a period of intensive innovation, this academic study addresses some of the common issues which arise when designing multidimensional measures of wellbeing in detail. By documenting the GNH Index methodology and findings we hope to share a tool which can be adapted by others engaged in the development of measurement tools that will advance GNH.

Let us begin by sharing a few of the key findings. Overall, in 2010, $8.3 \%$ of Bhutanese people are 'deeply happy' according to GNH; $32.6 \%$ are 'extensively happy'; $48.7 \%$ are 'narrowly happy', and 10.4\% are 'unhappy'. These four groups correspond to people who have achieved sufficiency in more than $77 \%, 66-76 \%, 50-65 \%$, and less than half of the nine domains, respectively. The 2010 GNH Index uses the middle cutoff. Its value is 0.743 and shows that, overall, $40.9 \%$ of Bhutanese are identified as happy (meaning they are extensively or deeply happy), and the remaining $59.1 \%$ enjoy sufficiency in $56.6 \%$ of the domains on average. Recall that $48.7 \%$ of these are already narrowly happy, but are considered not-yet-happy for policy purposes. GNH gradients and indices are reported for each of the 20 districts by gender, by rural-urban areas, and, for illustrative purposes, by age and certain occupational categories. Standard errors are presented, 
as are robustness tests for weights and cutoffs, measured with respect to district rankings and to the composition of insufficiencies.

The analysis has two parts: first, the wellbeing of the people who have been identified as 'happy' is examined to show the indicators in which they enjoy sufficiency. Some individual examples are presented to show that the 'happiest' people are diverse with respect to age, district, occupation, gender, and sufficiency profiles.

Second, the insufficiencies among those not identified as happy (or not-yethappy) are examined. The GNH Index value can rise either by increasing the percentage of people who are happy, or the percentage in which notyet-happy people enjoy sufficiency. This analysis clarifies areas where policy interventions or actions by other institutions could increase GNH. All tables used in this report, together with the survey instrument of questions used in the index and statistical analyses, are presented in the extensive appendices.

The GNH Index, like the philosophy of GNH which motivates it, is very much a living experiment with truth. It seeks to deploy rigorous scientific tools to convey more fully the colour and texture of people's lives than does the standard welfare measure of GDP per capita, to evolve the dimensions and the methodology of UNDP's Human Development Index, and to draw on innovative work from other initiatives seeking to measure human progress on a shared planet. 


\section{Introduction}

We strive for the benefits of economic growth and modernization while ensuring that in our drive to acquire greater status and wealth we do not forget to nurture that which makes us happy to be Bhutanese. Is it our strong family structure? Our culture and traditions? Our pristine environment? Our respect for community and country? Our desire for a peaceful coexistence with other nations? If so, then the duty of our government must be to ensure that these invaluable elements contributing to the happiness and wellbeing of our people are nurtured and protected. Our government must be human.

The Madhavrao Scindia Memorial Lecture delivered by His Majesty the King, 23 December 2009

\section{History of GNH as a Policy Priority}

Since 1972, as other countries clarified and focused their economies on material expansion, the then-Kingdom of Bhutan sought, through public action, to expand the wellbeing and true happiness of its people. The goal of Gross National Happiness - or GNH - was first articulated by the Fourth King, His Majesty Jigme Singye Wangchuck. He built upon the legacy of Bhutan's government since the 1729 legal code by Zhabdrung Rimpoche, which dates from the unification of Bhutan. The legal code stated that if the government cannot create happiness (dekidk) for its people, there is no purpose for the government to exist' (Ura 2010). The Constitution of Bhutan (2008, Article 9) directs the State 'to promote those conditions that will enable the pursuit of Gross National Happiness.' After the establishment of a constitutional monarchy in 2008 and the coronation of the Fifth King, the Government of Bhutan sought to specify this objective such that policies and programmes advanced by the new democracy continue to be coherent with it. This paper presents the rationale, methodology and results of one such specification: a multidimensional index of Gross National Happiness (henceforth the GNH Index).

\section{The Concept of GNH}

In his Coronation speech, the Fifth King, His Majesty Jigme Khesar Namgyel Wangchuck, said 'I have been inspired in the way I look at things by Bhutan's development philosophy of Gross National Happiness ... to me it signifies simply 'Development with Values." GNH at its core 
comprises a set of values that promote collective happiness as the end value of any development strategy. GNH might be described as:

- Holistic: Recognizing all the aspects of people's needs, be these spiritual or material, physical or social,

- Balanced: Emphasising balanced progress towards the attributes of GNH

- Collective: Viewing happiness to be an all-encompassing collective phenomenon

- Sustainable: Pursuing wellbeing for both current and future generations

- Equitable: Achieving reasonable and equitable distributed level of wellbeing

From these terms, the complexity of the concept is clearly seen. However, the greatness of the concept lies in its simplicity in giving priority to happiness and the term 'happiness' here reflects the creation of enabling conditions where people are able to pursue wellbeing in sustainable ways (Ura, 2009). This expresses the idea that happiness should be pursued as a common public good. Therefore, progress should be viewed not only through the lens of economics but also from spiritual, social, cultural and ecological perspectives. The concept of GNH has directed the country for four decades and exists as a guiding principle in the minds of Bhutanese and also as the overarching objective in almost all official documents of our country.

While there is no single official definition of $\mathrm{GNH}$, the following description is widely used:

Gross National Happiness measures the quality of a country in more holistic way [than GNP] and believes that the beneficial development of human society takes place when material and spiritual development occur side by side to complement and reinforce each other. ${ }^{1}$

From the start it is vital to clarify that GNH in Bhutan is distinct from the Western literature on 'happiness' in two ways. First it is multidimensional - not focused only on subjective wellbeing to the exclusion of other dimensions - and second, it internalizes responsibility and other-regarding motivations explicitly. As the first Prime Minister of Bhutan to be elected under the new Constitution of Bhutan adopted in 2008 put it:

${ }^{1}$ http://www.educatingforgnh.com/ 
We have now clearly distinguished the 'happiness' ... in GNH from the fleeting, pleasurable 'feel good' moods so often associated with that term. We know that true abiding happiness cannot exist while others suffer, and comes only from serving others, living in harmony with nature, and realizing our innate wisdom and the true and brilliant nature of our own minds. ${ }^{2}$

It includes harmony with nature (again absent from some Western notions of happiness) and concern for others. The brilliant nature he alluded to consists of the various types of extraordinarily sensitive and advanced awareness with which human beings are endowed and can be realized.

In Bhutan, Gross National Happiness represents a holistic set of values and priorities that are intended to guide public policy as well as institutions and agents across society. Like other complex objectives, GNH can be advanced many ways and by different actors. Primarily, GNH can be advanced by citizens in families and community activities, culture and sport, work and prayer. It can also be advanced by institutions from businesses to the entertainment industry to monasteries to the media to NGOs. The public sector at all levels also plays a vital role in advancing $\mathrm{GNH}$ and in supporting others' work to advance it.

One of several tools for public policies to advance GNH is an index of Gross National Happiness that enables policymakers to track progress across the different aspects of GNH. Caveats are natural: an index cannot include all aspects of GNH that are relevant. Nor is it sufficient to guide policy - it must be complemented by an in-depth, narrower analysis of policies and programmes, tailored to local realities. Further, it must be advanced by a plurality of institutions. Because advancing GNH depends upon actions by civil servants, government workers, the private sector, and civil society, the objective of maximising GNH must resonate with plural groups across Bhutanese civil service and society.

So while an index alone is limited and insufficient, a robust and compelling index - rigorously formulated and clearly presented - can do what no other single tool can do, which is sketch roughly how GNH is evolving across Bhutan as a whole over time, as well as for different groups, regions and people. It can also convey how people are happier - or unhappier than previously, and thus inform practical action.

${ }^{2}$ Lyonchhen Jigmi Y. Thinley. 'Opening address on Educating for Happiness'. 2009. 
If creating such an index were easy, it would already have been done. Yet just as Bhutan's objective of GNH has often captured the imagination of groups across the globe, so too Bhutan's work to develop a multidimensional index of wellbeing resonates with a number of concurrent initiatives. So the timing is apt to push forward such investigations. At the same time, modesty is required lest the claims for the GNH Index be greater than it can bear.

The current paper introduces the 2010 Gross National Happiness Index, which has been advanced by the Centre for Bhutan Studies under the leadership of Dasho Karma Ura. It provides a thorough explanation of the methodology, drawing attention to both strengths and standing questions. The remainder of this introduction describes the four pillars and nine dimensions of GNH and the purpose of the GNH Index for public policy. Part I of the paper sets out the methodology of the index. It introduces the 2008 GNH Index, the 2010 Gross National Happiness Survey, and the Alkire-Foster methodology as adapted for the GNH Index. After highlighting how the challenges of constructing a multidimensional welfare index were addressed in the GNH Index, it sets out the indicators, thresholds and weights and their justification. Part I concludes by presenting the GNH Index formulae and interpretation, and introducing the logic for the following two sections.

Part II focuses on understanding the achievements of people who have been identified as 'happy' because they enjoy a sufficient combination of achievements across domains - in this case 66\%. This includes the groups described as 'extensively happy' and 'deeply happy'. This section describes the GNH Index and its associated variables by district, age, gender, and other classifications, and shares the composition of sufficiency among happy people. Part III focuses on increasing happiness. It focuses upon people who are not-yet-happy (that is, those who are unhappy or narrowly happy) and scrutinizes the indicators and domains in which they lack sufficiency - because addressing these will increase GNH. This analysis uses the GNH Index, but focuses on the not-yet-happy population and on insufficiency rather than sufficiency.

\section{Pillars and Dimensions of $G N H$}

The $10^{\text {th }}$ plan of Bhutan specified GNH by focusing on four pillars: 'In order to translate the multidimensional concept of GNH into core objectives ... four strategic areas were initially defined' (p.16). These areas, called the 'four pillars of GNH', are: 1. Sustainable \& equitable socio-economic development; 2. Environmental conservation; 3. The preservation and promotion of culture; and 4. Good governance. 
Subsequently, nine dimensions of GNH were identified which specify the four pillars. The nine dimensions were selected on normative grounds and map more specifically the key areas of GNH. The dimensions are: psychological wellbeing, health, education, cultural diversity and resilience, time use, good governance, community vitality, living standard, and ecological diversity and resilience. The motivation for including each dimension - or domain as they are often called - is detailed below. As is apparent, three domains - living standard, health, and education - are traditional dimensions of public policy. Ecological diversity and good governance are more novel areas but are becoming common across many countries. The prominence of psychological wellbeing (which includes yet goes beyond subjective wellbeing), time use, community vitality and cultural diversity, is distinctive and innovative.

\section{Purpose of the 2010 GNH Index}

Since the mid-2000s, steps have been taken towards calculating a GNH Index which would draw as fully as possible on the holistic and deliberate vision of development as it has evolved in Bhutan. In a 2007 Government Round Table meeting, Dasho Karma Ura proposed that a GNH Index would be used in: 1. Setting an alternative framework of development; 2. Providing indicators to sectors to guide development; 3. Allocating resources in accordance with targets and GNH screening tools; 4. Measuring people's happiness and wellbeing; 5. Measuring progress over time; and 6. Comparing progress across the country. ${ }^{3}$ These purposes each have specific implications for measurement, which are elaborated below.

1. Setting an alternative framework of development. Bhutan's GNH vision of development is distinctively holistic. The $10^{\text {th }}$ plan explicitly seeks 'to address a more meaningful purpose for development than just the mere fulfilment of material satisfaction. ${ }^{34}$ Hence the nine domains of GNH, taken together, reflect the purpose of development. If certain dimensions contract, or are being crowded out by material progress, the GNH Index must explicitly convey such information as the imbalances enter, in order to catalyse public deliberation and if relevant, action.

2. Providing indicators to sectors to guide development. Certain indicators must either monitor activities by the public sector or else change when sector priorities are realized. For example 'electricity', a component of the GNH, is a priority in the $10^{\text {th }}$ five-year plan. Insofar as the GNH indicators

\footnotetext{
3 Royal Government of Bhutan 2008a

${ }^{4}$ Royal Government of Bhutan 2008b

10
} 
monitor outputs, the GNH Index provides incentives to ministries to deliver services, because their accomplishments will visibly contribute to higher GNH the next time the index is updated. Methodologically this requires an index that can be broken down into its component indicators.

3. Allocating resources in accordance with targets and GNH screening tools. While the composition of the GNH is not a sufficient guide for policy, a clear understanding of how the achievements and shortfalls in different dimensions of GNH vary over time and space and group provides key information for policy design and subsequent resource allocation. In terms of targeting, the GNH Index can show which dzongkhags (district) are lacking in which indicators, and can also identify and target the least happy' people and describe them by age, district, gender, etc. In terms of screening tools, the GNH indicators can be used as a check list to convey in concrete terms the kinds of activities and achievements that constitute $\mathrm{GNH}$.

4. Measuring people's happiness and wellbeing. The measure and its component indicators aim to capture human wellbeing in a fuller and more profound way than traditional socio-economic measures of economic development, human development or social progress have done. This also requires the measurement methodology be understandable to the general public. Case studies can be provided of differently happy people, in order that citizens can assess whether the index broadly seems intuitive and has room for their own aspirations and values.

5. Measuring progress over time. The component indicators of the GNH are to be sensitive to changes over time. Some indicators must be directly responsive to relevant changes in policy. In this way, the composition of wellbeing, as well as its overall level, can be observed over decades. Similarly, inequalities among groups, and populations that require special attention can be identified. The GNH Survey hence must be repeated regularly, for example every two years.

6. Comparing progress across the country. The GNH Index should be able to make meaningful comparisons across the dzongkhags, which vary widely in terms of climate, culture, access to services, and livelihoods. The survey hence must be representative by dzongkhag and the methodology of measurement must be subgroup consistent and decomposable.

Taken together these six requirements have been used to specify the indicators and composition of the GNH Index. It must be policy-sensitive - changing over time in response to public action to reflect strengthening or deterioration in the social, cultural, and environmental fabric whether or 
not at present these states are the direct objective of policy. In certain sectors, the indicators must reflect public priorities. The indicators must be assumed to be relevant in future periods as well as at the present time in order to measure progress across time. And the GNH Index must be subgroup consistent hence decomposable by regions and groups. 


\section{Part I: Construction of the 2010 GNH Index}

\section{Background}

The Royal Government of Bhutan in 2005 made the decision to develop $\mathrm{GNH}$ indicators to operationalize the concept of GNH. The indicators were to serve to ascertain whether programmes and policies were consistent with the values of GNH. The government intended to create conditions for evidence-based policy and for generating innovative policy and programmes to implement GNH. From 2005 the Centre for Bhutan Studies (CBS) involved nine researchers in developing the GNH indicators. In carrying out their responsibility to develop the indicators, CBS hosted extensive consultations at various levels ranging from private meetings with government officials and civil servants to focus group discussions with Bhutanese citizens.

In order to generate the pilot survey, CBS developed a detailed pre-pilot questionnaire covering the nine key areas considered crucial for reflecting the values and principles of GNH. These key areas of GNH fall within the domains of psychological wellbeing, health, time use, education, culture, good governance, ecology, community vitality and living standards.

After the consultations with stakeholders such as the sector heads of various agencies and the general public, CBS conducted an unusually extensive pilot survey in 2006 with 350 respondents. The pilot was used to design both the survey questionnaire and also the survey administration process. As would be expected, the pilot survey provided vital insights into the relevance of questions, translation problems, comprehension issues, accuracy and non-sampling error, and comparability across different respondents. Information on the range of response choices used, completion time etc. were also explored. The pilot questionnaire took four to seven hours to complete.

The findings of the pilot survey were analysed and shared with national leaders and academics, generating further consultations and discussions at director level and secretarial level in government to revise the indicators further.

\section{GNH Index}

The Centre for Bhutan Studies carried out the First Gross National Happiness survey in December 2007. The survey questionnaire included 
over 640 indicators, including objective, self-report, subjective, and openended questions. Due to budget restrictions the survey covered 950 respondents in 12 districts: Dagana, Tsirang, Wangdue Phodrang, Samtse, Zhemgang, Pemagatshel, Samdrup Jongkhar, Tashigang, Tashiyangtse, Gasa, Haa and Thimphu. It was representative at the national level. The enumerators usually required three to four hours to complete a questionnaire. $^{5}$

Drawing on that survey, each of the nine domains was analysed. Alongside that, to give an overview of the extensive and rich detail, the first GNH Index was developed. The 2008 GNH Index adapted the methodology of multidimensional poverty measurement by Alkire and Foster (2007, 2011a). It was constructed across the nine equally weighted domains, using 72 indicators. A person was identified as happy if they had achieved sufficiency in each one of the 72 indicators. No Bhutanese had achieved such sufficiency, and so analysis focused on the achievements enjoyed. A significant practical outcome of the index was to inform the policy and project screening tools, ${ }^{6}$ and also to sensitize researchers and research users to the possibilities of the index and of the demand for communications materials and policy-relevant analyses.

\section{Gross National Happiness Survey}

The 2010 GNH survey implemented a revised questionnaire containing over 750 variables. It built on the 2007 survey and repeated many questions exactly. In addition a further literature review was carried out intensively at CBS on the nine domains of GNH. Based upon this extensive literature survey, researchers identified additional relevant survey questions that were likely to be appropriate in the Bhutanese context.

Alongside the academic work, a participatory consultation process was used to access information from Bhutanese decision-making bodies so as to develop more effective GNH indicators. A two-stage, high level set of focus group discussions were organized by CBS: one at the director level and the other at the secretary level. Decision-makers shared their perspectives and priorities regarding the ongoing problems and issues which needed to be considered in programmes and policies. The participatory meetings included discussions regarding key value judgments

\footnotetext{
5 The 2007 survey data and instrument are available at www.grossnationalhappiness.com.

${ }^{6}$ The 2008 GNH Index did not stand alone. Rather, a set of project and policy screening tools were developed to complement and specify it for different purposes. See http://www.gnhc.gov.bt/wp-content/uploads/2011/05/Policy-and-Project-ScreeningTools.pdf. 
in the index construction. These discussions enabled certain indicators to be identified as particularly valuable and given priority in the questionnaire.

After finalization of the questionnaire, CBS trained 55 enumerators for three weeks in order that the 2011 GNH survey would be completed efficiently and to a high standard. The enumerators were divided into five teams, and each team was led by a field supervisor from CBS. The survey was fielded in April to December, 2010. The extensive field time was due to funding and to the scattered nature of settlements, which made it difficult for enumerators to interview more than one person in a day. Travel to survey locations was time consuming due to the remoteness of the villages and the geographical terrain, which is only partly served by roads. The fieldwork was monitored by five coordinators and an overall supervisor. Each team was assigned a set of primary sampling units (PSUs) across the country and interviews were conducted in the household of the selected PSUs with assistance from local government leaders. On an average, interviews took three hours each. Completed questionnaires were monitored and assessed by the five team coordinators, followed by the overall supervisor and then lastly by evaluators in the data entry division. Six data entry operators, who had previously been trained on questionnaire data entry and editing, undertook data entry and cleaning using the software Epi Info.

\section{Sample Design}

The 2010 Gross National Happiness Survey was conducted nationwide with representative samples from stratum (rural and urban) as well as districts. The sampling unit is the household and respondents are older than 14 years of age.

The initially targeted sample was 8700 and covered all 20 dzongkhags and all 202 gewogs $^{7}$ in Bhutan. The sample was drawn by National Statistics Bureau (NSB) as a sub-sample to the Bhutan Multiple Indicator Survey (BMIS) 2010 survey. That sample design can be found in Appendix A of the 2010 BMIS Report. The final GNH survey contains 7142 respondents and is nationally representative, representative by rural and urban areas, and by each of the 20 districts or dzongkhags. It covers respondents aged 15 to 98 with the mean of 41 years. Forty-eight per cent of the respondents are male and $52 \%$ are female.

7 The lowest administrative unit consisting of a number of villages. 


\section{Unit of Analysis and Data}

The unit of analysis of the GNH Index is the person. Hence all indicators must be present for each respondent. Any household-level variables such as income, housing, assets, and sufficiency or insufficiency in these are ascribed to the respondent; hence it is not possible to reflect intrahousehold inequalities in the household-level variables. While in practice indicators might be separately sourced and merged, in this context all indicators were drawn from the GNH Index Survey. In the process of data analysis, a number of observations emerged that will be used to improve the GNH Survey in the next period.

\section{Choice of GNH indicators: 13 trials}

GNH aims to create a society in which the collective happiness of the people is the ultimate desired outcome. The indicators will help to determine GNH policies and track GNH progress through time. So the indicators need to reflect all the relevant aspects of life which are vital to the concept and practice of GNH. But how many should there be, and how should they be chosen? This section gives a broad overview of the various steps required to select indicators according to more empirical and statistical criteria. The consultations with policymakers and with communities, as well as normative discussions, complemented these methods.

The 2006 survey questionnaire included data on more than 1000 variables; in 200872 of these variables, covering the nine domains of $\mathrm{GNH}$, were used to construct the 2008 GNH Index. In 2010, the GNH Index includes 33 indicators for the nine domains, which have been constructed using 124 variables. The selection of the 2010 indicators was informed by participatory consultations as well as by considerable empirical as well as theoretical work. This work included the construction of a range of alternative GNH indices prior to the selection of the final index.

Variable selection and indicator construction proceeded in stages. First, the cleaned dataset was discussed with the supervisors; questions were identified that had not been well-understood or were likely to be inaccurate and were discarded. Second, variables that had low response rates were identified and discarded. Questions that referred only to a subset of respondents were also set aside. These included questions asked only of parents of young children, or only to people who smoke, drink alcohol, or take doma for example. These questions can be used for supplemental analysis. Note that some questions that referred to rural quality of life (such as wildlife damage to crops) were retained and will be 
discussed shortly. Third, some variables did not relate to the respondents' own wellbeing, but rather sought their opinion regarding trends or institutions in Bhutan (which might not serve in one's local area) - such as satisfaction with school facilities or other people's values. Such questions were discarded as the connection to an individual's own happiness might not be direct and strong. Fourth, the indicators which are appropriate for an index of joint distribution must each be an arguably good proxy for that individual's attainment in the past period. Some indicators, particularly those with short recall periods such as morbidity in the last two weeks, are designed to be accurate on average across respondents, but may not reflect individual attainments in a longer period accurately. These were not used, with the exception of the 'time use diary' as discussed below. Other variables were demographic and so collected in order to analyse the data rather than to construct the index.

Of the variables in the survey, around 175 were retained for consideration in the index itself. Each candidate variable was then further studied to identify whether it was: a) objective or subjective or self-report; b) stock or flow; or c) resource, input, output, or outcome. Also, each variable was analysed to see how it related to public action - that is, to ascertain its policy relevance. The aim, thus, was to select variables for the GNH Index which were well-defined and, when possible, were policy-relevant outcome indicators, usually objective, which would show change across time. Such an index alone could fulfil the several purposes laid out above.

Prior to as well as after variable screening, exploratory factor analysis, ${ }^{8}$ cluster analysis, ${ }^{9}$ and correlation analyses ${ }^{10}$ were applied systematically

\footnotetext{
8 Factor Analysis reduces the data by consolidating it so as to structure around the covariance structures of the variables. It tries to combine variables that are overlapping and tries to separate out those that are not. For example, running factor analysis on emotional experience variables in the psychological wellbeing domain identifies emotional variables which are redundant and so is used to select variables which are distinct and cover the range of emotional experiences felt by people. Of course factor analysis (and related techniques for ordinal variables) is entirely statistical and must be complemented by analyses of the normative importance of variables.

${ }^{9}$ Cluster analysis is another possible way of looking at the similarity between variables according to some predefined criteria. It clusters together similar variables, up to the level of aggregation. For example, it might be used to propose clusters of variables that represent the same underlying functionings. It is a kind of extension of exploratory factor analysis since it uses the statistical information contained in the entire distribution and not only the covariance or correlation matrices of the data. For instance, the variables contained under the family relationship category were clustered to obtain a family relationship index.

10 A correlation is a single number that describes the degree of relationship between two random variables. Correlation is one of the most widely used data reduction techniques and has been used to reduce the chance of double counting similar attributes. The correlation
} 
across possible variables (both across all variables and within pre-defined domains) to identify statistical relationships and enable the categorisation of variables into domains as well as the selection of an optimal number of variables. Due to this analysis (as well as to the intuitions arising from the exercise) some variable adjustments were made. For example the general health questionnaire on mental health - which in 2008 had been categorised within psychological wellbeing - was re-categorised into the health domain. Similarly, questions on spirituality (prayer, meditation, and karma) - which had been in cultural diversity - were re-categorised into psychological wellbeing.

Having roughly explored the variables and domains, an extended process of generating trial indices was started. For example, on the basis of factor analysis with the re-categorised variables alone, two GNH indices were constructed and analysed in which the variables for each domain were selected simply on the basis of statistical association. Where there was a choice between indicators, enumerators' prior analyses of data quality were used to select the more reliable indicators. The two GNH indices differed in the number of indicators; in one, having 151 variables, the emphasis was to use every indicator that contributed and in the other, to reduce the number of indicators quite sharply - in that case down to 53 variables. Naturally later, in the creation of sub-indices, these statistical exercises were repeated to inform and justify the construction of particular indicators, as will be detailed below.

Alongside the shortlisting of potential variables was the issue of when to enter each variable into the GNH Index directly, and when to aggregate variables into a 'sub-domain' or complex indicator. Four main considerations shaped the final choice. The first was accuracy. In some cases - such as positive emotions - it seemed that aggregating the variables was likely to improve their accuracy. Second was policy relevance. In the Alkire-Foster methodology, if an indicator enters the measure directly, the measure can be broken down to that indicator level to show the censored headcounts and percentage contributions. On the other hand, if the variable is pre-aggregated into a sub-domain, then while the analysis can always describe the data, there is no simple decomposition; key distinctions were thus maintained. Third, in a number of cases the

coefficient may take on any value between plus and minus one. The sign of the correlation coefficient $(+,-)$ defines the direction of the relationship, either positive or negative. A positive correlation coefficient means that as the value of one variable increases, the value of the other variable increases and as one decreases the other decreases. A negative correlation coefficient indicates that as one variable increases, the other decreases, and viceversa. 
indicators were aggregated based on conventions (GHQ index) and/or statistical analysis and validity tests such as Cronbach's Alpha. Appendix 5 details the considerations exhaustively for each indicator. Fourth was communication. Because the purpose of the index does entails its communication to policymakers and the general public, the number of indicators was considered strategically. If, for example, the GNH Index had 175 indicators, its public comprehension might be quite low. Experiences with the 2008 GNH Index suggested that slightly fewer indicators might facilitate its use.

During the process of selecting indicators and thresholds, therefore, a large set of distinct GNH indices were developed, calculated, decomposed by groups, and analysed. This labour-intensive process underlies the development of the final GNH Index. The trial indices provided insights on the GNH survey data, on sensitivity of results to the choice of indicator, on whether radically different indices created radically different results and policy messages. It also allowed researchers to test empirically different procedures for indicator selection and interpretation and to understand their strengths and weaknesses. The trial indices contained:

1) 70 variables to match the 2008 GNH Index as closely as possible

2) 53 indicators selected by factor analysis

3) 151 indicators selected by factor analysis

4) 36 objective indicators ${ }^{11}$

5) 17 objective, outcome or output indicators

6) 15 objective, outcome or output indicators

7) 29 subjective indicators

8) 29 subjective indicators with lower sufficiency cutoffs applied

9) 32 indicators selected to suit the normative purposes of the index

10) 46 indicators from 167 variables selected to suit the normative purposes of the index

11) 54 indicators selected to suit the normative purposes of the index

12) 67 indicators using more variables selected to suit the normative purposes of the index

13) 173 indicators (including objective, subjective, outcome, input, resource, capability, etc.)

\footnotetext{
11 In all cases indicators for psychological wellbeing were subjective; the others, objective.
} 
The final GNH Index draws upon the analysis of these previous sets of indices, which serve also as robustness tests on the choice of indicators for the final index.

One last consideration must be considered separately, and that is the treatment of subjective data.

\section{Subjective and Objective Indicators}

The GNH surveys include subjective and objective questions. A difficult issue in constructing the GNH Index was whether, and if so how, to combine subjective and objective data. As has been mentioned, trial indices were constructed having only 'objective' indicators, only 'subjective' indicators, and both. These categories are put in quotations because they are not neatly distinct, as others have observed (Pudney 2011). Most papers analysing the use of subjective indicators advise these to be analysed and aggregated separately from objective indicators (Diener and Suh 1997; Cummins 2000, 2003; Rojas 2011); however, there are some recent exceptions (OECD 2010). Analyses of these results informed our decision to include some subjective indicators, but, in any dimensions other than psychological wellbeing in which such appear, to give them a lighter weight.

The decision to include psychological wellbeing as a dimension in the $\mathrm{GNH}$ is integral to its very definition, and so was not problematic in our view. Sen 2009 argues that satisfaction with one's life can be seen as an important functioning alongside other functionings, and Stiglitz, Sen and Fitoussi $(2009 \mathrm{a}, \mathrm{b})$ similarly argue that subjective wellbeing is one of the dimensions of quality of life. If it is understood as an intrinsically important functioning and if the indicators are sufficiently accurate, then it seems appropriate to include - particularly given Bhutan's policy priorities. Its inclusion could introduce concerns on the trade-offs between investing in materially and socially well-off people who have psychological or emotional needs, and investing in the materially poor. But these concerns are less likely to be realized in practice because the structure of the GNH Index will evaluate GNH across all nine domains, and only consider a person as unhappy if they have not attained sufficiency in six domains. And because the indicators of psychological wellbeing include emotional balance and spirituality as well as satisfaction, it may be relatively more revealing than standard subjective wellbeing questions.

Clearly both subjective and objective assessments are important and revealing for policy when properly analysed. The genuine issue is whether to 'mix' subjective and objective indicators in the GNH Index. The 
arguments against are clear: it is not certain that trends in subjective indicators such as self-reported health status, or perceptions of government performance, will be easily interpretable over time. Trends may not be easily interpretable because the indicators may be influenced by changes in the frame of reference, which is likely to occur as roads, electricity, literacy and connectivity increase. If trends are not interpretable, this would make the trend of the GNH Index over time less useful according to its stated purposes.

The subjective indicators were used 1) when the objective indicators did not sufficiently cover important aspects of a domain; 2) when the subjective indicators arguably did address the missing aspects, and 3) when the evidence from subjective preferences tracked what one would have expected from objective preferences. For example, we do use self-reported health status, despite the controversy about this indicator (Sen 2002). However in this case it is because the only health variables in the GNH survey otherwise are the days in the last month in which the respondent was healthy (which does not necessarily reflect their health over the past year or two), as well as their disability status. The self-reported health question functions to give an overall indication of health during a longer period; further evidence of adaptive preference is not evident, in that rural selfreported health is lower than urban, older is lower than younger, and so on. To prevent possible difficulties in trends of GNH, this indicator is given $10 \%$ of the domain weight and the other three indicators are allocated $30 \%$ of the domain weight each so $90 \%$ in total.

The following subjective questions are used in the GNH Index and, with the exception of those in psychological wellbeing, were attributed only $10 \%$ of their respective domain weight each:

Psychological wellbeing (all have a subjective element) ${ }^{12}$

Satisfaction

Positive and Negative Emotions

Spirituality

Health

Self-reported health

Governance

Government performance

Fundamental rights

Ecological Diversity and Resilience

Responsibility towards the environment

Perceptions of ecological issues.

${ }^{12}$ For the exact questions please see Appendix 2 
Hence the GNH Index does mix subjective and objective indicators, having eight subjective and 25 objective indicators, but it does so after extensive consideration of how to adjust the measure accordingly. Three of the eight subjective indicators comprise the dimension of 'psychological wellbeing.' The remaining subjective indicators receive only $10 \%$ of the weight of their respective domain or dimension, so together the subjective indicators count for $11 \%$ (one domain) plus $6 \%=17 \%$ of the GNH Index weighted indicators.

\section{Domains and Indicators}

Appendix 5 exhaustively explains each of the nine domains and 33 indicators of the GNH Index 2010, including how they have been constructed as well as the cutoffs that have been set and how they are variously justified. Table 1 provides an overview of the index and shows how many sub-domains or indicators have been constructed for each domain, for a total of 33 .

Table 1: Overview of GNH domains and indicators

\begin{tabular}{|l|l|c|}
\hline & Domain & Number of Indicators \\
\hline 1 & Psychological wellbeing & 4 \\
2 & Health & 4 \\
3 & Time use & 2 \\
4 & Education & 4 \\
5 & Cultural diversity \& resilience & 4 \\
6 & Good Governance & 4 \\
7 & Community vitality & 4 \\
8 & Ecological diversity \& resilience & 4 \\
9 & Living standards & 3 \\
\hline & Total & 33 \\
\hline
\end{tabular}

\section{Thresholds}

The GNH Index uses two kinds of thresholds or cutoffs: sufficiency thresholds and one happiness threshold. Sufficiency thresholds show how much a person needs in order to enjoy sufficiency in each of the 33 cluster indicators. It asks how much is enough to be happy. Each of the 33 cluster indicators has a sufficiency threshold and each person in the survey is identified as enjoying sufficiency or not in each indicator. How are these sufficiency thresholds set?

There were different inputs to calibrate these decisions. Some use relevant and appropriate international standards e.g. for hours of work and overcrowding in a house. Some use national standards e.g. a sufficiency income is equivalent to 1.5 times the income poverty line for Bhutan. For other indicators, there was no literature or precedent in Bhutan or 
internationally to set sufficiency thresholds. For this reason, some rely on value judgements, e.g. for positive emotions. In this case, the GNH thresholds are based on normative value judgements which have been shared and discussed in consultative sessions. The final and important inputs were participatory meetings. The Centre for Bhutan Studies held consultative conversations with different institutions and leaders in government, and focus group discussions with communities in different rural areas and sought their input, checking with them about the thresholds on test or trial GNH indices while the final GNH Index was still being finalized. And their insights proved very useful but also drew attention to the fact that no one set of thresholds will be accurate across all people in Bhutan. And that is why it is very important to have a second cutoff -- a sufficient happiness threshold which allows for a lot of variation between people, based on their own personalities and aspirations as well as on their material, community and climactic circumstances. All of the indicators with their cutoffs will not be equally meaningful or relevant in the many varied contexts of Bhutan - but they need not be. The second threshold permits diversity.

In reporting the GNH, we divide the population into four sub-groups by applying three cutoffs, which refer to people who have achieved sufficiency in $50 \%, 66 \%$, and $77 \%$ of the weighted indicators. This enables us to identify the unhappy, narrowly happy, extensively happy, and deeply happy. We can analyse each of these groups' achievements separately. For each person, we have their personal profile of achievements across all 33 cluster indicators, and these profiles provide a rich basis for analyses of these four different GNH Groups - the indicators and dimensions in which they lack sufficiency, and how these change by gender, region, age, and occupation.

To calculate the GNH Index, we choose one threshold or cutoff. We could choose the lowest cutoff in which case we would find that only $10 \%$ of Bhutanese were unhappy. However this would restrict the policy focus to a small set of the population, leaving the rest unsupported. So instead, we choose the middle happiness cutoff of $66 \%$. Thus the not-yet-happy group includes both those who are unhappy and those who are narrowly happy - a total of $59.1 \%$ of people. Our analysis of how to increase GNH' focuses on increasing the sufficiency of these groups.

This second cutoff is referred to as the happiness threshold. It is set across the nine domains and the 33 cluster indicators. The question that it asks is 'how many domains or in what percentage of the indicators must a person achieve sufficiency in order to be understood as happy'? Here it is important to acknowledge that this approach is an experiment. Happiness 
is a very deeply personal experience and any measure of it is necessarily imperfect. The index is offered to the people of Bhutan for understanding, discussion and debate to see if it frames and captures their understandings and how this might change or be improved.

The happiness threshold was set based on three criteria. The first is diversity as not all of the indicators have universal applicability. It may not be necessary to have sufficiency in all of the indicators to be happy e.g. a person who is very old might not need sufficiency in education indicators in order to be happy. They might have other members of their family who can read for them or explain things that require a formal education and their wisdom and skills may suffice for their own happiness. Some people, such as atheists for example, may not participate in prayer recitation or meditation.

The second is measurement error. Responses might not be completely accurate about peoples' values in different cultures - for example, people may be hesitant to say what exactly their beliefs or practices are for fear of seeming proud or ostentatious. Because of the difficulty of allowing for these differences, (as it is done in poverty measures) it seemed reasonable not to require sufficiency in every domain.

The third and last criterion is freedom of choice. Many people are fully happy without achieving sufficiency in every single indicator. Maybe they are not healthy but they have achieved a kind of flourishing, fulfilment and richness of life that is important. Maybe they are illiterate or have material challenges but that need not necessarily be decisive for their happiness. Thus to allow some freedom of choice we have set the happiness threshold at $66 \%$.

\section{Weights}

The weights of the GNH Index are a function of two features. The first is the explicit weight on each indicator. The second is the relative frequency of sufficiency in each indicator. The choice of weights relied on disparate inputs. These included the participatory discussions with national leaders mentioned above, focus group discussions and fieldwork with local communities, considerations of indicator reliability (elaborated below), and statistical analysis. This attempt to synthesize diverse inputs regarding weights means that the final choice of weights is arbitrary, and weights are best conceptualized as a 'range'. For this reason, the robustness of the GNH Index was tested for some ranges of weights with respect to the cross-indicator composition of GNH (used for policy response) as well as 
the dzongkhag categories, and these results are presented later. This section presents the explicit weights used.

The explicit weights are straightforward. Each of the nine domains is equally weighted, for the reason given above that the nine domains were identified so as to be relatively equal in normative importance. As the indicators had been selected to reflect a diversity of instantiations of each domain, ${ }^{13}$ the default weight was equal among indicators. However in two cases these were adjusted. The first case was already explained above and relates to the subjective indicators, which were given a very light weight due to uncertainty regarding their interpretability across time.

In addition, six questions were given $20 \%$ of the domain weight of their indicator due to concerns regarding measurement error. This can be justified as follows. It is in the nature of a household survey that many questions used are 'self-report'. For example, in the living standard domain people are asked to report their income, their livestock and landholdings, their asset holdings, the number of healthy days in the last month, and so on. The measurement error in these questions is well-documented and can be high. ${ }^{14}$

The GNH Index uses six questions whose 'self-report' feature may have the potential to have a larger measurement error. For example, in education, people were asked of their values about killing, stealing, and so on, but the question could be interpreted differently in ways that would allow persons having an identical set of values to answer them differently depending upon their interpretation. The 'family' questions were asked to the respondent out of earshot of family members, but despite this practice the answers may be biased by a concern on the part of respondents about being overheard, and so on. On these particular questions, we also applied a lighter weight $(20 \%)$. These questions are presented in Appendix 3 and can be summarised as follows:

\footnotetext{
13 The key exception to this is in education in which years of schooling and literacy were both included deliberately to give a higher relative weight to years of schooling among the literate population.

14 The measurement error in living standard questions was informally evident when comparing certain chiwogs where the households that had been interviewed for the GNH Survey had also been interviewed 6 months earlier in the 2010 BMIS questionnaire; questions such as assets, number of rooms, and housing materials even varied more than would be expected during the intervening period. A systematic comparison was not possible.
} 
Education

Knowledge questions

Value questions

Community vitality

Community questions

Family questions

Cultural diversity and resilience

Speak native language

Driglam Namzha

As in the case of the selection of indicators, in the trial GNH Indices, a range of different weighting structures were implemented. Furthermore, the final GNH Index was tested for robustness to changes in weights.

Table 2: Weights on the 33 indicators

\begin{tabular}{|c|c|c|}
\hline Domain & Indicators & Weight \\
\hline \multirow{4}{*}{$\begin{array}{l}\text { Psychological } \\
\text { wellbeing }\end{array}$} & Life satisfaction & $33 \%$ \\
\hline & Positive emotions & $17 \%$ \\
\hline & Negative emotions & $17 \%$ \\
\hline & Spirituality & $33 \%$ \\
\hline \multirow{4}{*}{ Health } & Self-reported health & $10 \%$ \\
\hline & Healthy days & $30 \%$ \\
\hline & Disability & $30 \%$ \\
\hline & Mental health & $30 \%$ \\
\hline \multirow{2}{*}{ Time use } & Work & $50 \%$ \\
\hline & Sleep & $50 \%$ \\
\hline \multirow{4}{*}{ Education } & Literacy & $30 \%$ \\
\hline & Schooling & $30 \%$ \\
\hline & Knowledge & $20 \%$ \\
\hline & Value & $20 \%$ \\
\hline \multirow{4}{*}{$\begin{array}{l}\text { Cultural diversity } \\
\text { and resilience }\end{array}$} & Zorig cbusum skills (artistic skills) & $30 \%$ \\
\hline & Cultural participation & $30 \%$ \\
\hline & Speak native language & $20 \%$ \\
\hline & Driglam Namzha (the Way of Harmony) & $20 \%$ \\
\hline \multirow{4}{*}{$\begin{array}{l}\text { Good } \\
\text { governance }\end{array}$} & Political participation & $40 \%$ \\
\hline & Services & $40 \%$ \\
\hline & Governance performance & $10 \%$ \\
\hline & Fundamental rights & $10 \%$ \\
\hline \multirow{4}{*}{ Community vitality } & Donation (time \& money) & $30 \%$ \\
\hline & Safety & $30 \%$ \\
\hline & Community relationship & $20 \%$ \\
\hline & Family & $20 \%$ \\
\hline \multirow{4}{*}{$\begin{array}{l}\text { Ecological diversity } \\
\text { and resilience }\end{array}$} & Wildlife damage & $40 \%$ \\
\hline & Urban issues & $40 \%$ \\
\hline & Responsibility towards environment & $10 \%$ \\
\hline & Ecological issues & $10 \%$ \\
\hline \multirow{3}{*}{ Living standards } & Per capita income & $33 \%$ \\
\hline & Assets & $33 \%$ \\
\hline & Housing & $33 \%$ \\
\hline
\end{tabular}


Table 2 provides the weights applied for each of the 33 indicators in the GNH Index. All the weights on indicators for one domain sum to $100 \%$. As is evident, the relative weight on work and sleep is the highest of all indicators, at $50 \%$ of one domain, or $1 / 18^{\text {th }}$ of the total weight. The next four most highly weighted indices relate to political participation and services in governance, and wildlife damage and urban issues in ecological diversity. While these indicators receive a higher weight due to the presence of subjective indicators in that domain, they are also normatively justifiable. Political participation and the delivery of public services are the key aspects to governance, with the first reflecting citizen participation and the second reflecting the success of the service delivery. In ecology, wildlife damage is the overwhelming concern in rural areas, as was stressed also by communities in the participatory fieldwork component of this study. The indicator of urban environmental issues - traffic congestion, a lack of green areas, a lack of pedestrian facilities, and urban sprawl provide some insight into key issues at present, but will need to be adjusted as urbanization concerns evolve.

Having presented the indicators, domains, and weights, we now introduce the methodology by which the GNH Index was constructed, first by identifying who is happy using a happiness cutoff, and then by ascertaining the share of indicators in which not-yet-happy people enjoy sufficiency.

\section{Alkire-Foster Methodology}

The Gross National Happiness Index is constructed by building innovatively upon the simple, rigorous and decomposable methodology for measuring poverty developed by Alkire and Foster $(2007,2011)$ that can be used to measure poverty or wellbeing. It is a robust method which identifies a group - in this case those people who are not-yet-happy (vs. those who are happy) by considering the 'sufficiencies' they enjoy. It is a flexible method which has been fully tailored to the needs and context in Bhutan. This includes identifying the happiness gradient - the four population subgroups according to the percentage of weighted indicators in which they have sufficiency.

Like other measures in the Alkire-Foster family, the GNH Index is created from two numbers:

i. Headcount ratio: percentage of people who are happy

ii. Breadth: percentage of domains in which people who are notyet-happy enjoy sufficiency (this is similar to 'intensity' in poverty measures using the Alkire-Foster method) 
We first describe the approach intuitively and subsequently present more formal notation.

To construct the GNH Index using this methodology the following steps are followed:

i. Choose indicators

ii. Apply sufficiency thresholds (who has enough)?

iii. Apply weights for each indicator

iv. Apply the Happiness Gradient to identify four categories of Bhutanese

v. Select the middle cutoff as the happiness threshold and identify two groups:

1. Happy people (extensively and deeply happy)

2. Not-yet-happy people (policy priority) (unhappy and narrowly happy)

vi. Identify among the not-yet-happy people, in what percentage of domains they lack sufficiency, and in what percentage they enjoy sufficiency.

vii. Calculate the GNH Index and its associated statistics

This section presents the last four steps.

\section{Sufficiency Cutoff}

The first step is to define whether each person has attained sufficiency in each of the indicators. This is done by applying a sufficiency cutoff to each indicator. This is a novel step. In poverty measurement, a poverty cutoff is applied in order to distinguish poor from non-poor people or households. Poverty thresholds are imperfect and arbitrary, but the concept is wellunderstood. A sufficiency cutoff functions like a poverty or deprivation cutoff, but is set at a higher level. A person is identified as having a sufficient attainment if his or her achievements in that indicator meet or exceed the cutoff. Appendix 5 described the 33 indicators that have been chosen for the GNH Index, as well as the sufficiency cutoffs for each indicator.

If a person has achieved sufficiency, then their actual attainment is replaced by the value of the sufficiency cutoff. For example, if a perpetual student had been studying for 30 years and the sufficiency cutoff were 21 years, then the perpetual student would be treated as if they had 21 years of education. Achievements above the sufficiency cutoff do not further increase GNH. The level at which the sufficiency cutoff is set is a value judgment, which can be a topic for public discussion, but the fact that it may be difficult to set an exact cutoff should not obscure the reasonableness of setting some sufficiency cutoff. In the 2010 GNH Index, 
various sufficiency cutoffs were applied to different trial indices before choosing the final set; similarly, multiple cutoffs may be applied in order to detect a range of changes - for example among those exceeding the sufficiency cutoff(s) or those who have achieved less.

\section{Happiness Gradient}

Having identified whether a person has sufficiency in each of the indicators, the next question is how to identify certain people as 'happy'. A key reason to identify people as happy because they have realized sufficiency in some percentage of domains is to emphasize human diversity. A person is not required to achieve sufficiency in all indicators in order to be happy. This is a tremendously important point: some people achieve genuine flourishing while in a state of material poverty, or despite being excluded from community events, or despite ill health, or without being well educated. Indeed a person who is fully flourishing in some percentage of domains may be as happy as a person who has attained nearly all: above a certain level, more does not necessarily matter.

Just as within each dimension, we set sufficiency cutoffs to say 'that this much is enough' so too across dimensions we set a second happiness cutoff to say 'this much is enough'. It is sufficient - sufficiency in additional indicators may enhance some people's lives and will affect the gradient, but the happiness cutoff identifies people who, it is assumed, have sufficient achievements to be happy.

While in a poverty measure, only one or at most two cutoffs are used normally - one to identify the poor and occasionally a second to identify the extreme poor - in the case of the GNH measure, again because it is innovative and data rich, three cutoffs were selected and applied in order to generate four categories of people, each identified according to the percentage of domains or weighted indicators in which they had achieved sufficiency. The first cutoff identifies who is unhappy because they enjoy sufficiency in less than $50 \%$ of indicators; the second identifies the narrowly happy - those who enjoy sufficiency in half to two-thirds of indicators $(50-66 \%)$. The third identifies the moderately happy - those who enjoy sufficiency in $66-76 \%$ of indicators. The last identifies the deeply happy, who enjoy sufficiency in $77 \%$ of indicators or more. 


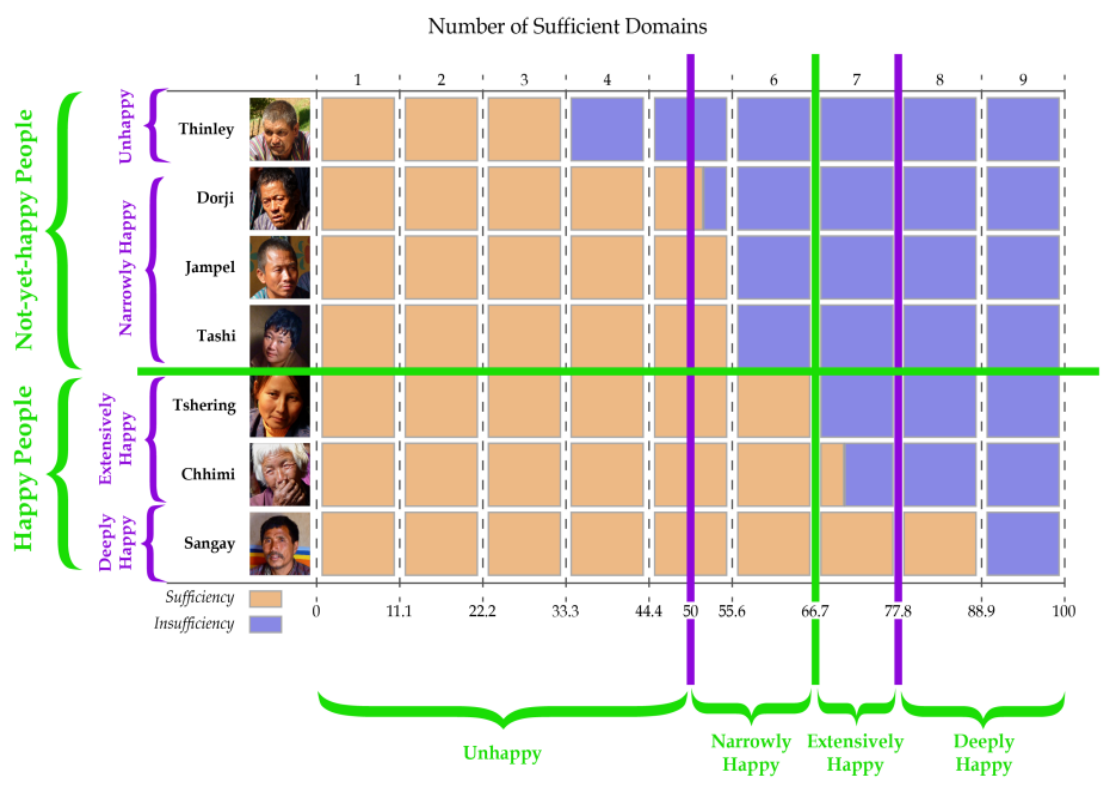

Percent of Sufficient Domains

Figure 1: Happiness gradient

As figure 1 shows, when we apply the $50 \%$ cutoff we find that only one person, Thinley, is unhappy. Looking between $50-65 \%$ we find three people are narrowly happy: Dorji, Jampel and Tashi. Two people have sufficiency in $66-76 \%$ of domains: Tshering and Chhimi. And finally, one person, Sangay, is deeply happy with achievements in over $77 \%$ of domains. We can compute the average sufficiency for each group also: for example, in the case of the narrowly happy people, the average sufficiency is $[(4.6 / 9+5 / 9+5 / 9) / 3]=54 \%$.

Yet, as a policy tool, it must be very easy to communicate the results of the GNH Index. When the GNH Index is updated, the government must be able to report whether the percentage of people who are happy has increased or decreased over time, where most change has occurred, and what dimensions and indicators increased and decreased. For this reason, one of the three cutoffs was used - the middle cutoff - for the GNH Index.

\section{Identification for the GNH Index}

So the 2010 GNH Index identifies a person as happy if he or she has attained sufficiency in $66 \%$ or more of the weighted indicators, which is equivalent to six of the nine domains. Thus the GNH identifies a person 
as not-yet-happy if he or she lacks sufficiency in more than $33 \%$ of indicators. ${ }^{15}$ Those who are extensively or deeply happy are understood to enjoy GNH; in contrast, the not-yet happy group includes those who are unhappy or narrowly happy.

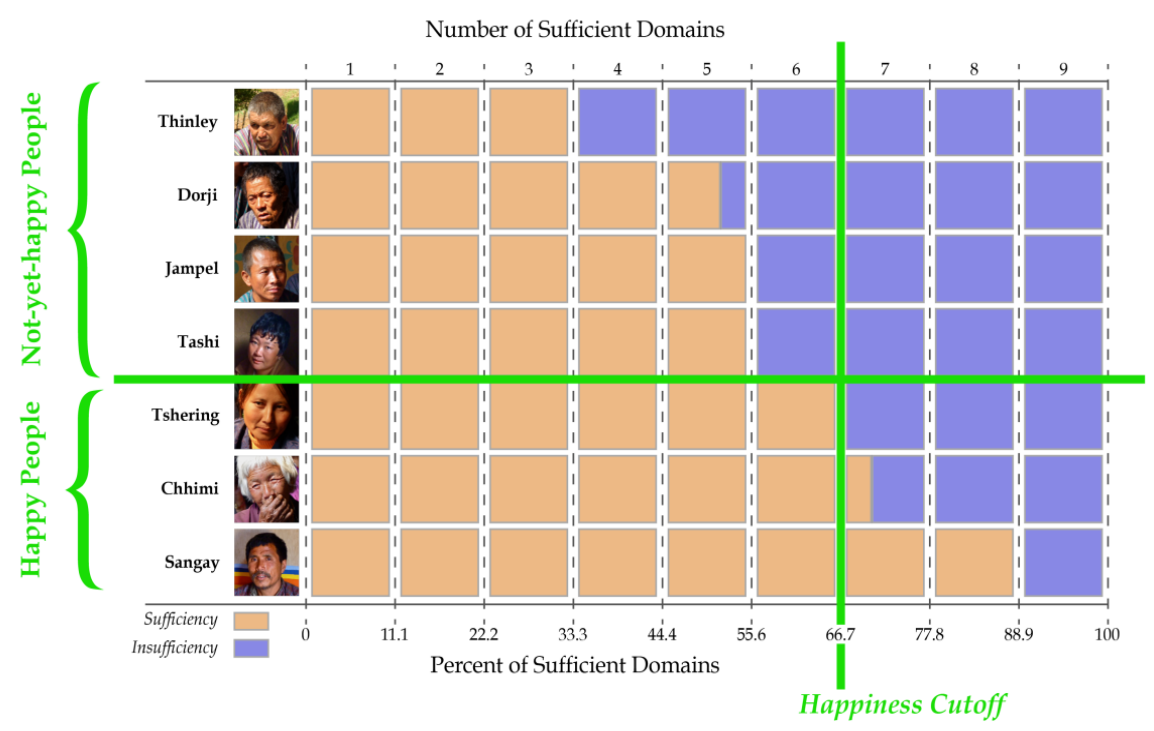

Figure 2: Identifying who is happy according to the GNH

Figure 2 uses an illustrative sample of seven people with nine domains to show how step 6 works in practice (to identify - among the not-yet-happy people - the percentage of domains in which they lack sufficiency, and in what percentage they enjoy sufficiency). ${ }^{16}$ The people at the top have sufficiency in the fewest domains, while those at the bottom have the most.

15 It would also be possible to construct a GNH Index simply to describe different sufficiency levels and compositions, but not claim any person to be happy. Such an approach could still provide examples of how different people achieve sufficiency in a different set of indicators, and so show the diversity of experiences and achievements which create GNH. Yet this approach is more complex to explain and also runs the danger of seeming to respect diversity less. That approach might also give equal policy importance to increasing the attainment of the happiest person as of the least happy person, which could be morally troubling as well as inefficient. In essence, this approach would use a 'union' identification techniques, and because the $H=100 \%=1$, all of the focus would be on the proportion of domains in which each person or representative group enjoyed sufficiency.

${ }^{16}$ Note that this is a simplification: the actual calculation uses 33 indicators and calculates an individual deprivation profile based on these rather than only nine domains, but the same principles apply. 


\section{Sufficiency and Insufficiency among the Not-Yet-Happy}

How do we move from this picture to the GNH? Here four out of seven people are not yet happy $-4 / 7=57 \%$, while 3 out of 7 people are happy $-3 / 7=43 \%$. Once we have this figure, to compute the GNH Index, we only need to know one more thing: Among the not-yet-happy people, in what percentage of domains do they enjoy sufficiency?

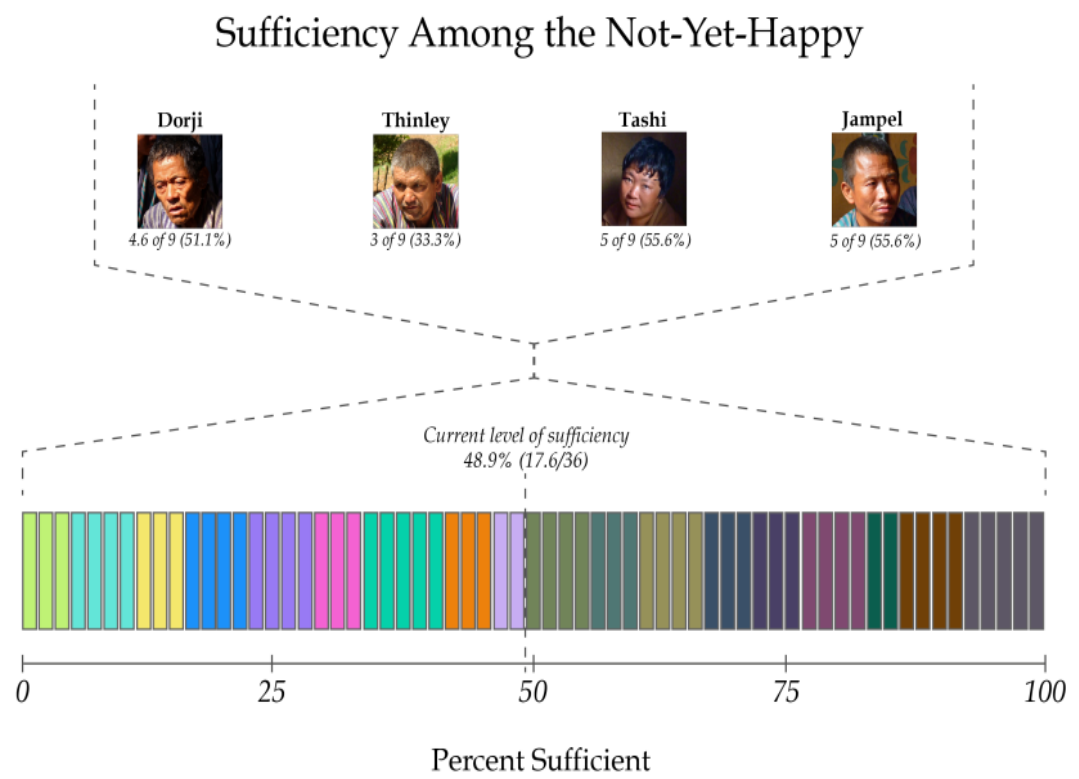

Figure 3: Calculating the percentage of domains in which not yet happy people lack sufficiency

The next step is to scrutinize the overall achievements of the not-yethappy people. We do this by taking the average among the not-yet-happy people of the proportion of weighted indicators in which they lack sufficiency. Figure 3 shows how we arrive at this figure. The not-yethappy lack sufficiency in $51.1 \%$ of domains, and enjoy it in $48.9 \%$ of domains in this example.

\section{Calculating the GNH Index}

To calculate the GNH Index, the data of the population are aggregated into a decomposable 'Adjusted Headcount $M_{0}$ ' measure that is sensitive to the 'breadth' of achievements (Alkire and Foster 2007). It is constructed by multiplying $H A$, where $H$ is the beadcount and represents the percentage of people who have not achieved sufficiency in 6 domains thus are identified 
as not-yet-happy, and $A$ is the average proportion of dimensions in which those not-yet-happy people lack sufficiency.

The Adjusted Headcount ranges in value from 0 to 1, with larger numbers signifying greater insufficiencies and less happiness. In order to create the GNH Index in which a higher number reflects greater happiness, the Adjusted Headcount is subtracted from 1 to obtain the GNH. GNH $=1$ $H A$.

The GNH Index formulae can also usually be written $\mathrm{GNH}=\mathrm{H}^{\mathrm{H}}+(\mathrm{H} \mathrm{x}$ $\left.A^{\text {Suf }}\right)$, where $\mathrm{H}^{\mathrm{H}}$ are the percentage of happy people $\left[\mathrm{H}^{\mathrm{H}}=(1-\mathrm{H})\right]$ and $\mathrm{A}^{\text {Suf }}$ is the percentage of dimensions in which the average not-yet-happy person nonetheless enjoys sufficiency $\left[A^{\text {Suf }}=1-A\right] \cdot{ }^{17}$ This way of presenting the same results focuses on happiness and sufficiency; the other focuses on the per cent of not-yet-happy and their insufficiencies; both are useful and will be drawn upon in later sections of the analysis. The value of the GNH Index is the same no matter which presentation is used.

We now present the methodology more formally.

\section{Methodology: GNH Index}

Let $M^{n, d}$ denote the set of all $n \times d$ matrices. The typical element $y \in M^{n, d}$ is the matrix of achievements of $n$ people in $d$ different dimensions. For every $i=1,2, \ldots, n$ and $j=1,2, \ldots, d$, the typical entry $y_{i j}$ of $y$ is individual $i$ s achievement in dimension $j$. The row vector $y_{i}=\left(y_{i 1}, y_{i 2}, \ldots ., y_{i d}\right)$ contains individual $i$ 's achievements in the different dimensions; the column vector $y_{\cdot_{j}}=\left(y_{1 j}, y_{2 j}, \ldots, y_{n j}\right)^{\prime}$ gives the distribution of achievements in dimension $j$ across individuals. Let $z_{j}>0$ be the sufficiency cutoff value in dimension $j$. The sum of entries in any given vector or matrix $v$ is denoted by $|v|$, while $\mu(v)$ is used to represent the mean of $v$ (or $|v|$ divided by the number of entries in $v$ ).

For any matrix $y$, it is possible to define a matrix of deprivations from sufficiency $g^{0}=\left[g_{i j}^{0}\right]$, whose typical element $g_{i j}^{0}$ is defined by $g_{i j}^{0}=1$

${ }^{17}$ This is a very simple re-arrangement as follows: $\mathrm{GNH}=1-\mathrm{HA}=1-\mathrm{HA}-\mathrm{H}+\mathrm{H}=(1-$ $\mathrm{H})+(\mathrm{H}-\mathrm{HA})=$

$(1-\mathrm{H})+(\mathrm{H})(1-\mathrm{A})=\mathrm{H}^{\mathrm{H}}+\left(\mathrm{HxA}^{\text {Suf }}\right)$, since $(1-\mathrm{H})=\mathrm{H}^{\mathrm{H}}$ and $(1-\mathrm{A})=\mathrm{A}^{\text {suf. }}$. 
when $y_{i j}<z_{j}$, and $g_{i j}^{0}=0$ when $y_{i j} \geq z_{j} .18$ That is, the $i j^{t h}$ entry of the matrix is 1 when person $i$ has not achieved sufficiency in dimension $j$, and 0 when he/she has sufficient.

For each of the $d$ dimensions we apply a weighting vector $\omega_{d}$ such that $\sum_{1}^{j} \omega_{j}=1$. The insufficiency profile of person $i$ is then generated by summing the weights of the dimensions in which person $i$ has not achieved sufficiency.

Following the methodology to identify the multidimensionally poor proposed by Alkire and Foster (2007), let $\rho_{k}$ be the identification method such that $\rho_{k}\left(y_{i}, z\right)=1$ when $c_{i} \geq k$, and $\rho_{k}\left(y_{i}, z\right)=0$ when $c_{i}<k$. That means that a person is identified as not having achieved happiness if he or she does not have sufficiency in at least $k$ dimensions. Once identification is applied, a censored matrix $g^{0}(k)$ is obtained from $g^{0}$ by replacing the $i^{\text {th }}$ row with a vector of zeros whenever $\rho_{k}\left(y_{i}, z\right)=0$. This matrix is used to generate the GNH Index and to analyse how happiness might be increased.

To construct the GNH Index, we first construct an Adjusted Headcount, given by $M_{0}=\mu\left(g^{0}(k)\right)$, which is the sum of the weighted indicators of those people who do not enjoy sufficiency in any indicator $\left(\left|g^{0}(k)\right|\right)$ divided by total the number of people $(n)$. It can also be expressed as $H A$ where $H$ is the Headcount Ratio $H=H(y ; z)$ defined by $H=q / n$, where $q$ is the number of people in set $Z_{k}$. A is the average percentage of dimensions in which people who are not yet happy experience insufficiency, and is given by $A=|c(k)| /(q)$. $M_{0}$ summarises information on the incidence of unhappiness and the average proportion of dimensions in which a not yet happy person lacks sufficiency. It satisfies dimension monotonicity and is also decomposable by population groups.

The GNH is constructed by subtracting $M_{0}$, from unity; that is, it is GNH $=1-M_{0}$.

18 Note that in some cases the sufficiency cutoffs are identified as weak rather than strong; this is explained in the domains and indicators section. 
The measure $M_{0}$, like all members of the $M_{\alpha}(y ; z)$ family, are decomposable by population subgroups. Given two distributions $x$ and $y$, corresponding to two population subgroups of size $n(x)$ and $n(y)$ correspondingly, the weighted average of sum of the subgroup poverty levels (weights being the population shares) equals the overall poverty level obtained when the two subgroups are merged:

$$
M_{0}(x, y ; z)=\frac{n(x)}{n(x, y)} M_{0}(x ; z)+\frac{n(y)}{n(x, y)} M_{0}(y ; z)
$$

Clearly, this can be extended to any number of subgroups such as dzongkhags, women and men, rural and urban, and so on.

Additionally, once the identification step has been completed, the $M_{0}$ index can be broken down into indicator. To see this, note that $M_{0}$ can be expressed in the following way: $M_{0}(y ; z)=\sum_{i=1}^{n} \mu\left(g_{*_{j}}^{0}(k)\right)$, where $g_{*_{j}}^{0}$ is the $j^{\text {th }}$ column of the censored matrix $g^{0}(k)$. Thus $\left(\mu\left(g_{*_{j}}^{0}(k)\right)\right) / M_{0}(y ; z)$ is the contribution of indicator $j$ to the overall shortfalls in GNH. Itemizing these shortfalls clearly provides information that can be useful for government policy.

\section{The GNH Index: Formulae and Interpretation}

The 2010 GNH Index value is $\mathbf{0 . 7 4 3 . ~ T h e ~ p e r c e n t a g e ~ o f ~ p e o p l e ~ w h o ~ a r e ~}$ happy is $40.9 \%$ and correspondingly, those who are not-yet-happy comprise $59.1 \%$ of the population. The intensity of sufficiency among those who are not-yet-happy is $43.4 \%$. Recall that the formulae for the GNH Index is $\mathrm{GNH}=1-M_{0}=1-(\mathrm{HxA})$. So the value is computed as follows

2010 GNH Index $=1-(0.591 \times 0.434)=0.743$.

This headline index has a direct intuition which is as follows. For the 'happy' people, we treat them as if they had achieved sufficiency in all domains - so the GNH Index among happy people is naturally $100 \%$. For the 'not-yet-happy' people, we identify the share of dimensions in which they have achieved sufficiency on average. Recall that the GNH Index formulae can also be written $\mathrm{GNH}=\mathrm{H}^{\mathrm{H}}+\left(\mathrm{H} \mathrm{x} \mathrm{A}^{\text {Suf }}\right)$ : the percentage of people who are happy plus the percentage of those who are not-yet-happy 
times the average sufficiency among the not-yet-happy. Numerically, this is:

2010 GNH Index $=40.9 \%+(59.1 \% \times 56.6 \%)=74.3 \%$.

In words, $40.9 \%$ of the people have achieved happiness, and the remaining $59.1 \%$ of people enjoy sufficiency in an average of $56.6 \%$ of the dimensions. The sum of these two figures is also $74.3 \%$.

It is easy to understand the GNH Index. It is also easy to see how it can be increased over time. If the percentage of people who are happy rises, the GNH Index will rise. For example what happens if the percentage of happy people rises to $42 \%$ and the average sufficiency of the not-yethappy is the same? It is easy to see that happiness will rise. For example, if the percentage of happy people increases to $42 \%$, this by definition means that the percentage of not-yet-happy people decreases to $58 \%$, because the number of happy and not-yet-happy people together add up to $100 \%$.

$\mathrm{GNH}=42 \%+(58 \% \times 56.6 \%)=74.8 \%$

We see that if the percentage of happy people $\mathrm{H}^{\mathrm{H}}$ rises, GNH rises.

Also, if the average sufficiency among not-yet-happy people rises, then the GNH Index will rise. For example, what happens if sufficiency rises to $60 \%$ but the percentage of happy and not-yet-happy people are still $40.9 \%$ and $59.1 \%$ respectively? The formulae is then

$\mathrm{GNH}=40.9 \%+(59.1 \% \times 60 \%)=76.4 \%$

We see that if the average sufficiency among the not-yet-happy (A ${ }^{\text {Suf }}$ ) rises, GNH rises.

In this way the GNH Index has a very simple and direct interpretation, and is sensitive to important changes in society over time.

But the real excitement of the GNH Index emerges in going inside of it, to understand its composition, and how achievements in different indicators vary between different regions and groups. The remainder of this paper analyses the GNH Index results. Part II analyses people who are happy in order to understand happiness in Bhutan at present, and Part III analyses how to increase happiness in Bhutan, by analysing people who are not-yethappy; the composition of insufficiencies, and policy implications to increase GNH. In order to present those results it is necessary to explain the methodology which underlies the analyses in the two subsequent parts of this paper. 


\section{Two Analyses: Understanding Happiness and Increasing Happiness}

Recall that the $\mathrm{M}_{0}$ methodology underlying the GNH Index can be decomposed and can generate a set of useful and consistent statistics including $\mathrm{H}$ and $\mathrm{A}$, as well as the censored headcounts, the per cent contributions of each indicator, and these figures for population subgroups. ${ }^{19}$ In a poverty measure, analysis focuses on poor people, the composition of their poverty, the per cent contribution of deprivations in different dimensions, and so on. Analogously, in Part III we analyse those who are not identified as 'happy' and the dimensions in which they lack sufficiency, using the statistics for the $\mathrm{M}_{0}$ measure, remembering that sufficiency cutoffs have been used, and that the range of domains is more extensive than usual.

Given the unique focus of the GNH Index, Part II analyses those who are happy according to the index, and the composition of their happiness. This is particularly useful in the stage of index design in order to analyse the index itself, as well as to explore the diversity in patterns of achieved happiness and adjust the indicators and cutoffs to better reflect chosen combinations of achievements. The analysis presented in Part II requires a methodological innovation which is explained below.

Recall the original $\mathrm{g}^{0}$ matrix. In order to analyse happiness we generate a corresponding $\mathrm{g}^{\mathrm{OS} \text { uf }}$ matrix, in which a person is given a value 1 if they have achieved sufficiency in that indicator - that is, if $y_{i j} \geq z_{j}-$ and 0 otherwise. The sufficiency matrix $\mathrm{g}^{0 \mathrm{Suf}}$ is the mirror of the deprivation matrix: for every 0 in the deprivation matrix there is a 1 in the sufficiency matrix, and for every 1 in the deprivation matrix there is a 0 in the sufficiency matrix. The same weighting vector is applied to the sufficiency matrix as was applied to the deprivation matrix. The weighted achievements of each person are then summarised in the $c_{i}^{S u f}$ vector, which shows the proportion of dimensions in which each person has attained sufficiency.

We then apply the corresponding identification function, which identifies a person as happy if they have achieved sufficiency in $k^{\text {suf }}$ dimensions where $k^{\text {suf }}=(1-k)$ dimensions. The identification function such that a person is identified as happy if $c_{i}^{\text {suf }}>k^{S u}$. The new identification function $\rho^{H}$ is then defined as $\rho^{H}(y ; z)=1$ if person $i$ is happy and $\rho^{H}\left(y_{i} ; z\right)=0$ if person $i$ is notyet-happy. When it is applied to the goSuf matrix, we create the censored matrix $\mathrm{g}^{0 \mathrm{Suf}}(k)$, in which the data of all people who are not-yet-happy are

19 Alkire and Foster 2007, Alkire and Foster 2011a, Alkire and Foster 2011b, Alkire and Santos 2010. 
censored - given a value of zero - and only the data of the happy people remain. We then can examine the sufficiency profiles of happy people using the same indices that we would analyse for the $\mathrm{g}^{0}(k)$ matrix. In this case $\mathrm{H}^{\mathrm{H}}=(1-\mathrm{H})$ and reflects the percentage of people who are identified as happy.

The GNH Index thus has associated with it a great richness of possible analyses, which will probably be simplified over time but are explored extensively in this first analysis. It is vital to be very alert and clear as to which analyses refer to which matrix. We have done this by creating a complete separation to avoid confusion. All of the analysis in Part II without exception is derived from the $\mathrm{g}^{\mathrm{OSuf}}(k)$ matrix, and all of the analysis of in Part III refers to the $\mathrm{g}^{0}(k)$ matrix.

The overall happiness cutoff provides a guide, a suggestion, which seems useful for the purposes to which the GNH Index will be put. At an individual level, happiness is a profoundly personal endeavor, and in practice people will continue to seek it in different ways. The measurement of GNH must continue to evolve so as to reflect the experiences of diverse citizens as fully and accurately as is required. To facilitate this interchange between the GNH Index and citizens' observations from their own lives, alongside the GNH Index it can be useful to provide profiles of happy Bhutanese who live very different lives: rural, urban; young, old; male, female; wealthy, modest; modern, traditional; Eastern, Southern; and so on. These can be used to stimulate public discussion and can feed into improvements of the GNH Index over time. 


\section{Part II. Understanding Happiness}

The GNH value is 0.743 . It shows us that $40.9 \%$ of people in Bhutan have achieved happiness, even with the structure of the GNH Index requiring a wide array of conditions to be met. Those who are not-yet-happy enjoy it in $56.6 \%$ of the domains, i.e. have sufficiency in $56.6 \%$ of the 124 weighted conditions. Happiness according to the GNH is reached when people reach sufficiency in roughly six out of the nine domains or the equivalent proportion of conditions. How can we deepen our understanding of these results?

The GNH Index provides an overall picture of how GNH is distributed in Bhutan and can be used to zoom in to look at who is happy and those that are not-yet-happy, and to zoom further to look at unhappy, narrowly happy, extensively happy, and deeply happy. The GNH can also be unpacked in different ways to tell different stories. It can be decomposed by subgroups like dzongkhags, age groups, gender, or some occupations. It can also be analysed by each dimension and indicator. All of these functions make it a useful tool for policymakers as they seek to address the question of 'how can GNH be increased?'

Overall, most Bhutanese enjoy sufficiency in value, safety, native language, family, mental health, urbanization issues, responsibility towards environment, satisfaction in life, government performance, healthy days and assets. Between $50-60 \%$ of Bhutanese enjoy sufficiency in ecological issues, negative emotions, community relationship, artisan skills and Driglam Nam₹ha. Less than half of Bhutanese enjoy sufficiency in literacy, housing, donations, work, services, schooling, cultural participation and knowledge.

Each of the GNH indices is also reported for each of the 20 districts, by gender, by rural-urban area, and, for illustrative purposes, by age and certain occupational categories. Standard errors are presented, as are robustness tests for weights and cutoffs, measured with respect to group rankings and also, for the first time, with respect to the percentage contribution of each indicator.

The 2010 GNH Index can be used to understand who is happy in Bhutan and to see the diverse profiles of happiness that different people enjoy. Based on the Alkire-Foster methodology, the GNH Index also provides an incentive to sustain GNH among the happy. In this section we first present the happiness gradient. Then we give some overall introduction to sufficiencies in Bhutan, as well as describe the domain composition of GNH. Then we decompose the GNH Index by subgroups like 
dzongkhags, age groups, gender, and some occupations. These comparisons and decompositions provide the texture and details of how people are happy.

\section{Results across the Happiness Gradient}

Recall that three cut off points have been used to identify degrees of happiness. Not all people need to be sufficient in each of 124 variables or 33 indicators to be happy. People are diverse in the ways and means they can have fulfilling life. People have freedom of choice in which ways they can make life fulfilling, so not all variables have universal applicability. For such reason, we divide the Bhutanese into four groups depending upon their degree of happiness. We use three cutoffs: $50 \%, 66 \%$, and $77 \%$. People who have achieved sufficiency in less than $50 \%$ are 'unhappy', and they comprise only $10.4 \%$ of the population. A total of $48.7 \%$ of people have sufficiency in $50-65 \%$ of domains and are called 'narrowly happy'. A group of $32.6 \%$, called 'extensively happy', have achieved sufficiency in 66$76 \%$ - in between 6 and 7 domains. And in the last group, 8.3\% of people are identified as 'deeply happy' because they enjoy sufficiency in $77 \%$ or more of weighted indicators - which is the equivalent of 7 or more of the nine domains.

Table 3 below presents the definition of each of the groups used in this analysis. It then gives the percentage of the population who belong in each category in the 2010 GNH Index results. The final column provides the average percentage of weighted indicators, or domains, in which people in each group, on average, enjoy sufficiency.

Table 3: Categories of GNH, Headcounts and Sufficiency

\begin{tabular}{|l|c|c|c|}
\hline & $\begin{array}{l}\text { Definition of } \\
\text { groups } \\
\text { Sufficiency in: }\end{array}$ & $\begin{array}{l}\text { Per cent of } \\
\text { population } \\
\text { who are: }\end{array}$ & $\begin{array}{l}\text { Average Sufficiency of } \\
\text { each person across } \\
\text { domains }\end{array}$ \\
\hline Happy & $\mathbf{6 6 \%} \% \mathbf{- 1 0 0 \%}$ & $\mathbf{4 0 . 8 \%}$ & $\mathbf{7 2 . 9 \%}$ \\
Deeply Happy & $77 \%-100 \%$ & $8.3 \%$ & $81.5 \%$ \\
Extensively Happy & $66 \%-76 \%$ & $32.6 \%$ & $70.7 \%$ \\
Not-Yet-Happy & $\mathbf{0 - 6 5 \%}$ & $\mathbf{5 9 . 1 \%}$ & $\mathbf{5 6 . 6 \%}$ \\
Narrowly Happy & $50 \%-65 \%$ & $48.7 \%$ & $59.1 \%$ \\
Unhappy & $0-49 \%$ & $10.4 \%$ & $44.7 \%$ \\
\hline
\end{tabular}

We can look across this happiness gradient by dzongkhag, gender and age, to obtain an idea of how it develops. The description below refers to table IX in Appendix 6.

The percentage of people who are 'deeply happy' is highest in Punakha $(15.7 \%)$ followed by Sarpang (15.3\%) and Paro (14.6\%); the percentage of 
deeply happy people is lowest in Tashigang (3.8\%) followed by Samdrup Jonkhar $(4.5 \%)$. On the other hand, the percentage of 'unhappy' people is highest in Samdrup Jongkhar (18.6\%) followed by Trongsa (15.6\%), and the rates of unhappiness are lowest in Paro (4.0\%) and Haa (5.5\%). Thus the Dzongkhags with highest and lowest GNH Index values similarly have consistently the highest and lowest rates of happiness and unhappiness. Interesting, the intensity - the percentage of domains in which unhappy people have sufficiency - are between $44-46 \%$ for all districts except Bumthang, in which unhappy people are mildly less unhappy, having sufficiency in $47 \%$ of the weighted indicators. Similarly, the intensity of sufficiency among the deeply happy is $80-82 \%$ for all districts except for extra-happy Dagana, with $82.7 \%$. This suggests that there is, at least at this time, less marked inequality across districts that there would be if the differences in intensity mirrored the differences in rates of unhappiness and deep happiness.

By gender, the differences are striking. $11.1 \%$ of men are deeply happy, and $37.4 \%$ of men are extensively happy, compared with only $5.4 \%$ of women who are deeply happy and $27.7 \%$ who are extensively happy. Among women, $52.5 \%$ are narrowly happy, and fully $14.3 \%$ are unhappy; in comparison, $45 \%$ of men are narrowly happy and only $6.5 \%$ are unhappy. The differences in intensity are, again, mild - which is a good thing!

By age, interestingly, the percentage of deeply happy people is relatively constant at $8-9.5 \%$ except among those aged $31-35$ and $>60$, in which only $7.1 \%$ and $3.8 \%$ respectively are deeply happy. However, a marked difference across age can be seen in unhappiness. Among those under 20, only $5.1 \%$ are unhappy, whereas for all those above 40 years it is over $11 \%$, and among those over 60 it is around $18 \%$ of people who are unhappy. This trend raises many questions. One possibility is that the younger generations are genuinely better off than their elders. This seems definitely part of the story because deprivations in education and living standards are markedly higher as the respondent age increases. If this is the case, then we will see this downward trend in GNH tapering off in the future as more Bhutanese enjoy education and higher living standards. A second possibility is that this trend reflects a need for services and support for the elderly, perhaps because the care in families is diminishing in strength. A third possibility is that the GNH Index domains like 'health' are such that, naturally, the aging process will correspond with lower sufficiency - and indeed health insufficiencies are highest among the elderly. However again on the positive side, deprivations in community vitality, in culture, and in psychological well-being are lower as people age 
- which might suggest a different worrying trend, namely an increase in insufficiencies in the very domains that make the GNH index innovative.

\section{Domain Composition of GNH}

The remainder of this section analyses the GNH index itself in different ways. Table 4 presents the domain composition of the GNH. All nine dimensions contribute to GNH meaning that happy people live relatively balanced lives without any dimension being unimportant. Among the nine dimensions good health $(14 \%)$, community $(12 \%)$, ecology $(12 \%)$, and psychological wellbeing $(12 \%)$ contribute the most to the GNH of happy people in 2010. Happy Bhutanese did not necessarily have high education $(9 \%)$. Nor did they score highly in good governance $(9 \%)$.

Table 4: Understanding bappiness - contributions

\begin{tabular}{ll} 
& $\begin{array}{l}\text { Percentage contribution of sufficiency } \\
\text { of each domain to overall happiness }\end{array}$ \\
\hline Psychological wellbeing & $11.97 \%$ \\
\hline Health & $14.07 \%$ \\
\hline Time Use & $10.45 \%$ \\
\hline Education & $9.06 \%$ \\
\hline Cultural diversity and resilience & $9.91 \%$ \\
\hline Good governance & $9.32 \%$ \\
\hline Community vitality & $11.83 \%$ \\
\hline Ecological diversity and resilience & $12.11 \%$ \\
\hline Living standards & $11.27 \%$ \\
\hline Total & $\mathbf{1 0 0 \%}$ \\
\hline
\end{tabular}

Although health and community vitality contribute equally to overall happiness, the sufficiency, and happiness structures with respect to the indicators composing these two domains differ. Figure 4 presents the percentage of people enjoying sufficiency, and figure 5 presents the percentage of people who are happy and enjoy sufficiency in each of the indicators. With respect to health we see that $89 \%$ of Bhutanese either do not suffer from long-term disability or have a disability but are not restricted in performing their daily activities, $86 \%$ of Bhutanese have normal mental wellbeing, $76 \%$ of Bhutanese have sufficient number of healthy days, and $74 \%$ of people in Bhutan have rated their health as either 'good' or 'very good'. Of the Bhutanese who achieved sufficiency levels in disability, 39\% are happy (fulfil the happiness threshold); of the those who have normal mental wellbeing, $39 \%$ are happy; of those who have achieved sufficient levels of healthy days, $36 \%$ are happy; and of the Bhutanese who have sufficiency in self-reported health status, $35 \%$ are happy. As none of these are $41 \%$, we also see that some people who do not have good health indicators are nonetheless happy. 
Compared to health, in community vitality the proportion of people enjoying indicator-sufficiency is higher. In this case $96 \%$ of Bhutanese have never been victims of crime, $93 \%$ of Bhutanese report good family relationships, $76 \%$ of Bhutanese report good community relationships, and $46 \%$ of Bhutanese provide donations at the sufficiency level (time and money). However, compared to health, the percentages of people who enjoy sufficiency and are happy are lower for each of the indicators composing community vitality. Thus, $39 \%$ of Bhutanese who report good family relationships are happy, $27 \%$ of Bhutanese who have good community relationships are happy, and $21 \%$ of Bhutanese who have sufficiency in donations (time and money) are classified happy. Hence 'happy' people in a number of cases nonetheless may not have sufficiency in community vitality indicators, particularly donations.

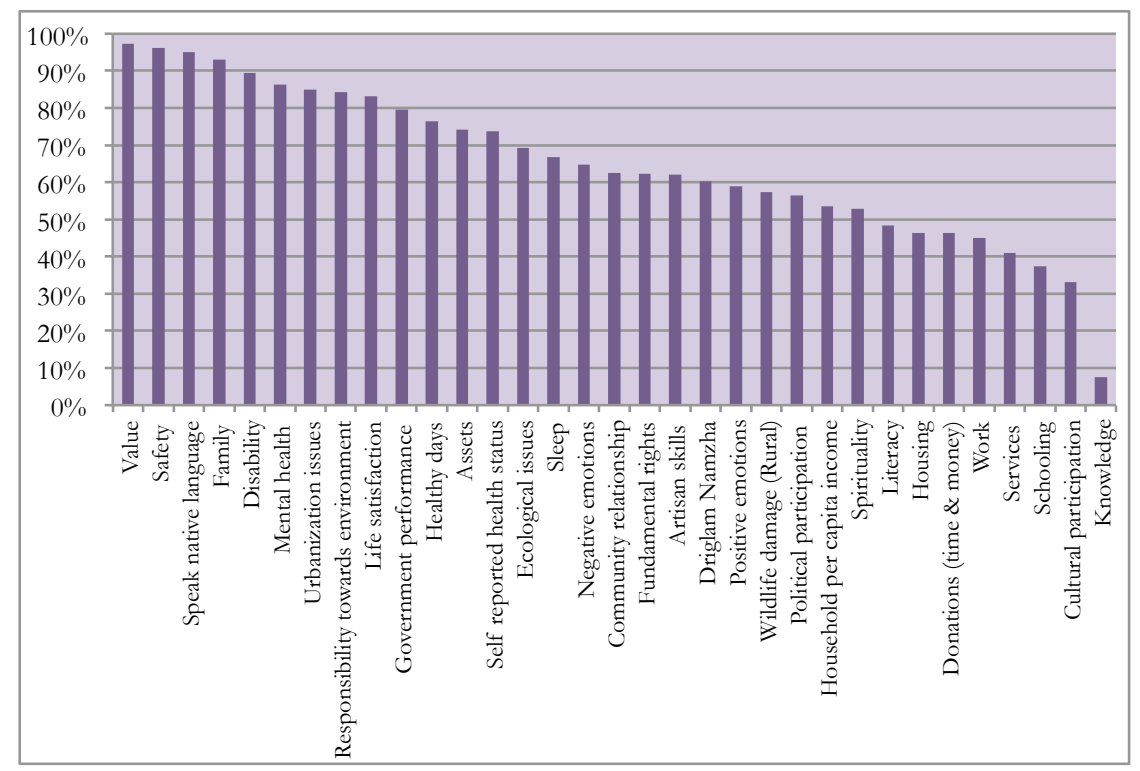

Figure 4: Percentage of people enjoying sufficiency

Overall in terms of indicators happy Bhutanese still often lack sufficiency in knowledge, participation in festivals, donations, having more than six years of schooling, enjoying government services, participating politically, and belief in the practice of Driglam Namzha (Figure 5). However they enjoy highest sufficiency in value, safety, native language, family, mental health, among others. 


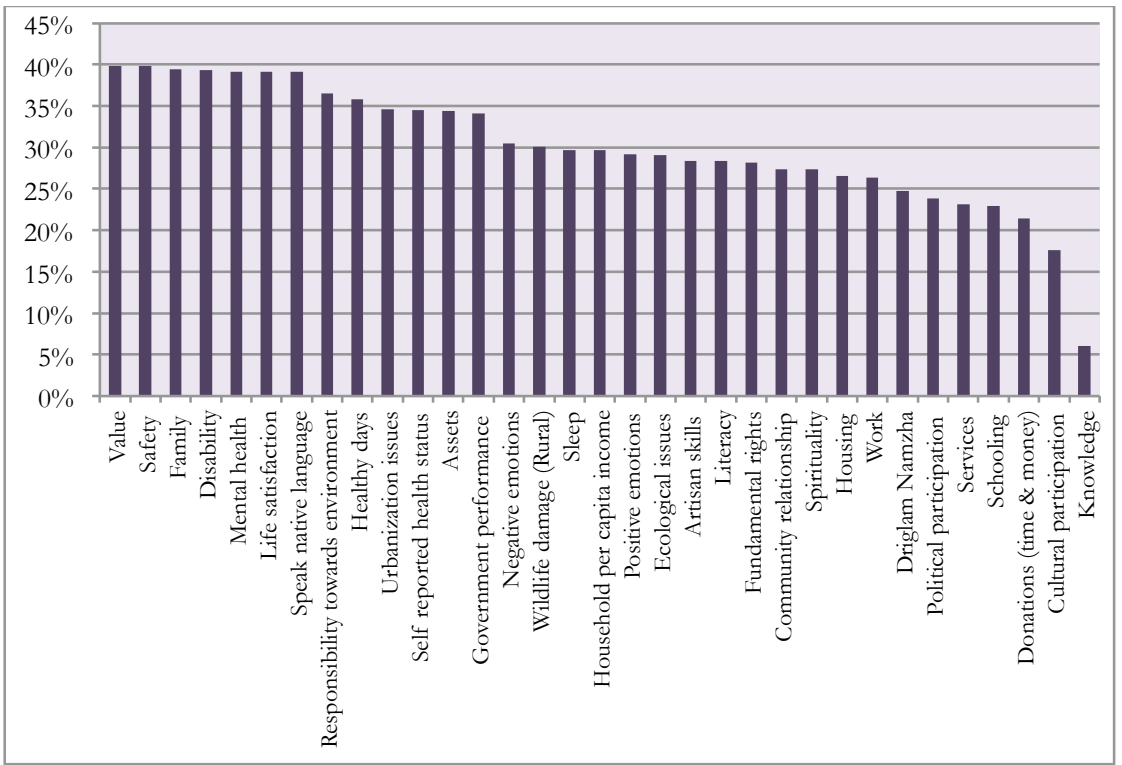

Figure 5: Percentage of people who are happy and have sufficiency

\section{Happiness by Drongkhag}

Figure 6 presents the GNH Index by dzongkhag. The districts are classified into three categories of happiness: low, medium, and high. Low levels correspond to districts with a GNH Index value between 0.655 and 0.706, and comprise Trongsa, Lhuntse, Tashiyangtse, and Samdrup Jongkhar. In contrast, Samtse, Chhukha, Wangdue Phodrang, Bumthang, Zhemgang, Mongar, Tashigang, and Pemagatshel, are districts belonging to the medium category of happiness. Their GNH values range between 0.707 and 0.756. Finally, districts from the west -- Haa, Paro, Thimphu, Punakha, Gasa- and from the south --Dagana, Tsirang, Sarpang--are classified in the high category of happiness and show values of GNH between 0.757 and 0.807 . This GNH classification is also reflected in the percentage of happy people by district. Thus, districts with low values of GNH are home to $24 \%$ to $34 \%$ of happy people. Those with medium values group have 35 to $44 \%$ of happy people. Lastly, districts with high values of GNH comprise 45 to $54 \%$ of happy people. 


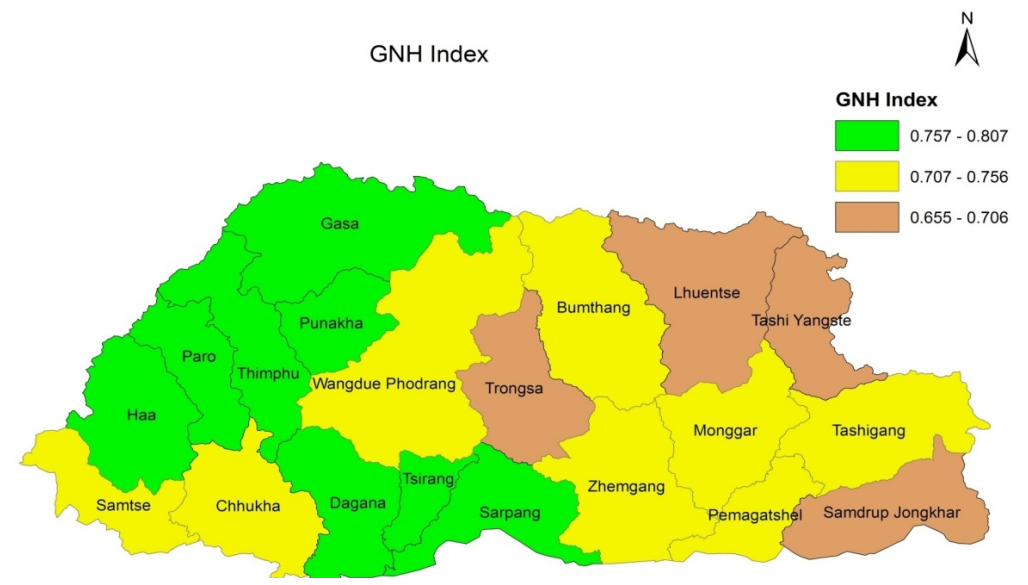

Figure 6: GNH Index by dzongkhag

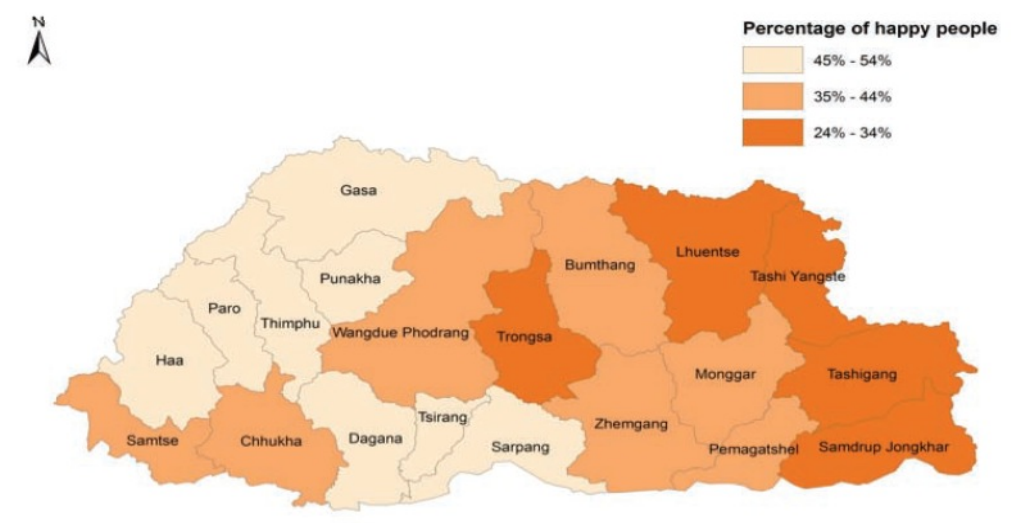

Figure 7: GNH Headcount by dzongkhag

Table 5 reports the values of the GNH Index by district. As is shown, all are above 0.655. Within categories however there is some variability. To get a clear idea of the variation of happiness within districts belonging to a given category, we report their confidence intervals in Figure 8. We see that Gasa, Haa, Tsirang and Paro are the districts with the largest confidence intervals. These four districts belong to the high category of happiness, indicating that high values of happiness should be interpreted with caution, as these are more dispersed. Within the medium category the values of the GNH Index by district exhibit more or less the same variation, with confidence intervals more or less of the same size. In the low category group Samdrup Jongkhar is the district with the tightest confidence interval among the low group, and also among all districts. 
Table 5: GNH values and categories by drongkhag

\begin{tabular}{llllll}
\multicolumn{2}{l}{$\begin{array}{l}\text { High category } \\
\text { District }\end{array}$} & GNH value & Dedium category & \multicolumn{3}{l}{ Low Category } \\
\hline Paro & 0.807 & Zhemgang & 0.753 & Lhuntse & 0.698 \\
Sarpang & 0.795 & Chhukha & 0.752 & Tashiyangtse & 0.698 \\
Dagana & 0.783 & Wangdue Phodrang & 0.737 & Trongsa & 0.684 \\
Haa & 0.775 & Samtse & 0.736 & Samdrup & 0.655 \\
Thimphu & 0.773 & Bumthang & 0.734 & & \\
Gasa & 0.771 & Mongar & 0.732 & & \\
Tsirang & 0.770 & Pemagatshel & 0.712 & & \\
Punakha & 0.770 & Tashigang & 0.708 & & \\
\hline
\end{tabular}

Confidence intervals of district level GNH indices

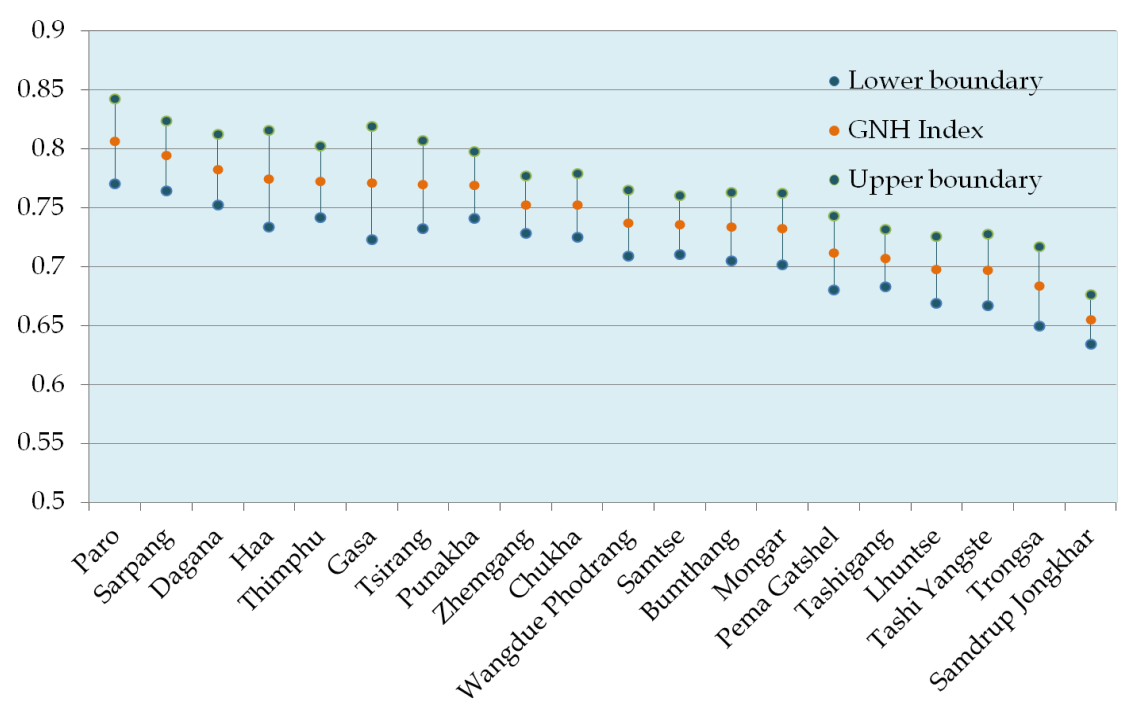

Figure 8: Confidence intervals of district level GNH indices

When compared with per capita income, GNH ranks districts differently than does per capita income (Figure 9). Thimphu (the capital) is not ranked highest in GNH terms, yet it has the highest per capita income of any dzongkhag in Bhutan, while Dagana and Zhemgang do much better in GNH than the income criterion. 
GNH Index $\quad-P$ Per capita income (BLSSR, Monthly Nu 2007)

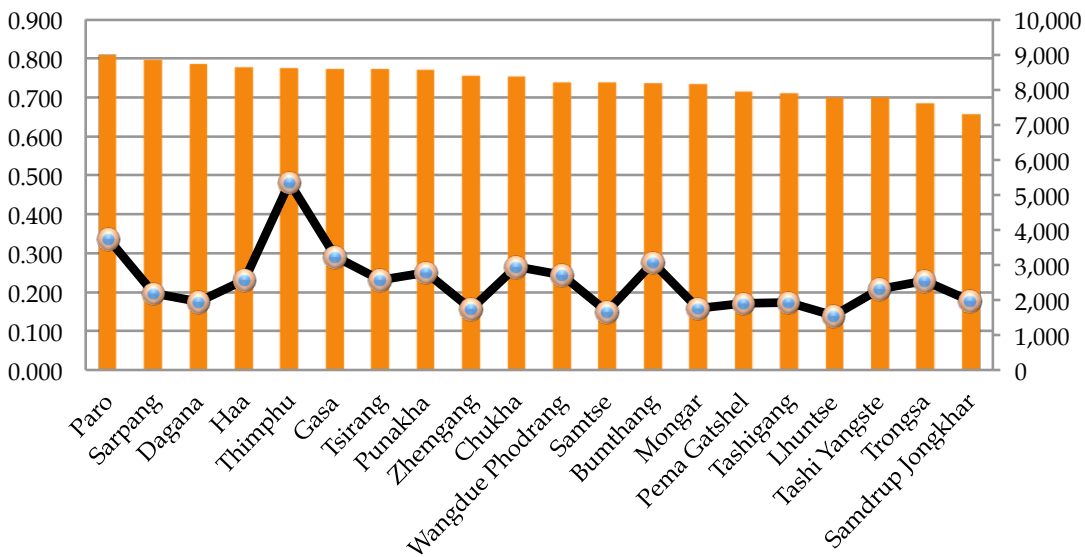

Figure 9: GNH Index and per capita income by dzongkhag

In terms of the domain contribution to the GNH by district, table 6 indicates that the composition of happiness changes a little across dzongkhags. Thimphu does better in terms of education and living standards, but worse in community vitality. Thimphu and Chhukha are also home to the highest number of happy people - and the highest number of not-yet-happy people (they are the biggest two dzongkhags in terms of population) in absolute terms. 
Karma Ura, Sabina Alkire, Tshoki Zangmo \& Karma Wangdi

Table 6: How the nine domains contribute to happiness by drongkhag

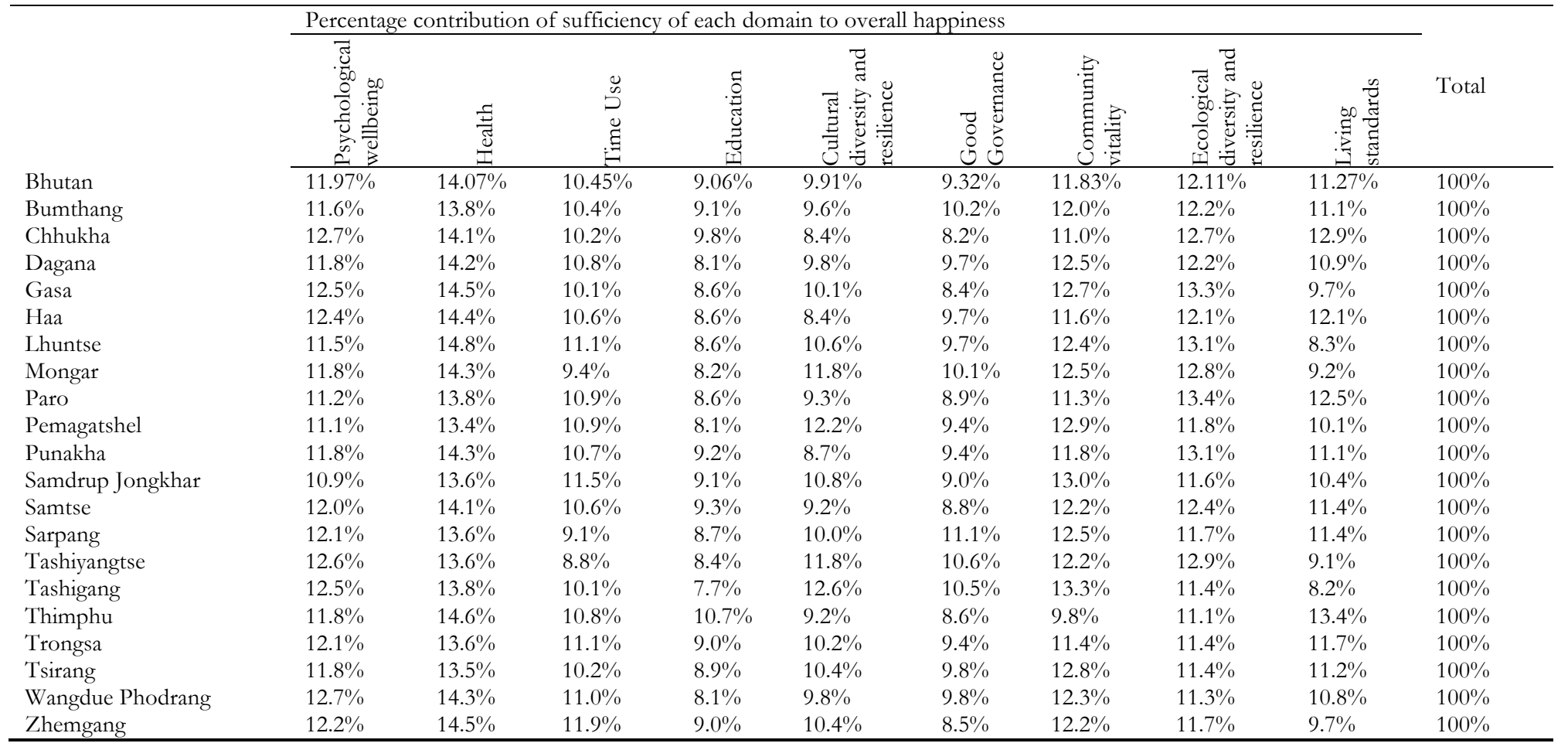




\section{GNH by Region}

Table I in Appendix 6 displays the GNH values by region. In general rural people are less happy than urban people but it is rather balanced. Fifty per cent of urban dwellers are happy by the GNH Index, whereas only $37 \%$ are in rural areas. Compared to national values the GNH Index in the urban sector is $7.1 \%$ higher, while the rural index is $1.5 \%$ lower. The contribution of domains to happiness also differs by region. Figure 10 presents the spider diagram associated with these contributions by region. In rural areas, community vitality, cultural diversity and good governance contribute more to happiness. In contrast, living standards, education and health contribute more to happiness in urban areas. Urban people experience insufficiency in governance, time use and culture, while in rural areas insufficiency is worst in education and living standards.

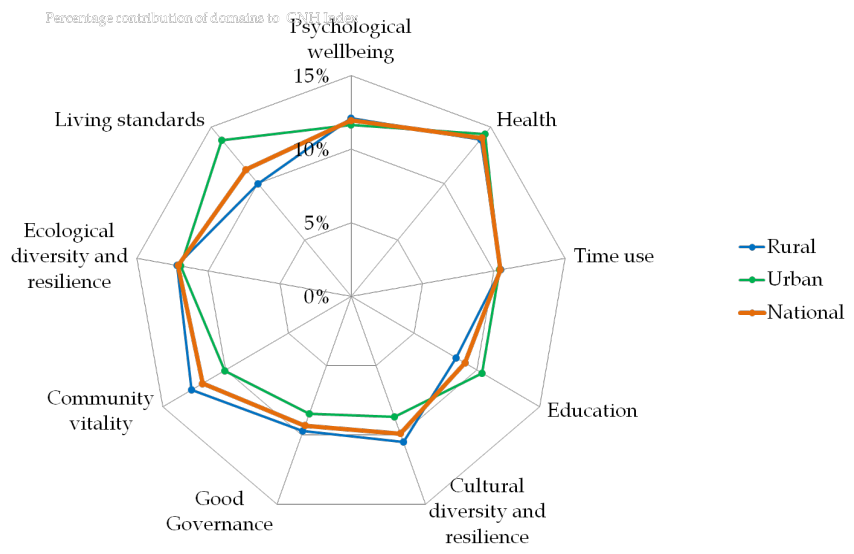

Figure 10: Contribution of domains to happiness by region

\section{GNH by Gender}

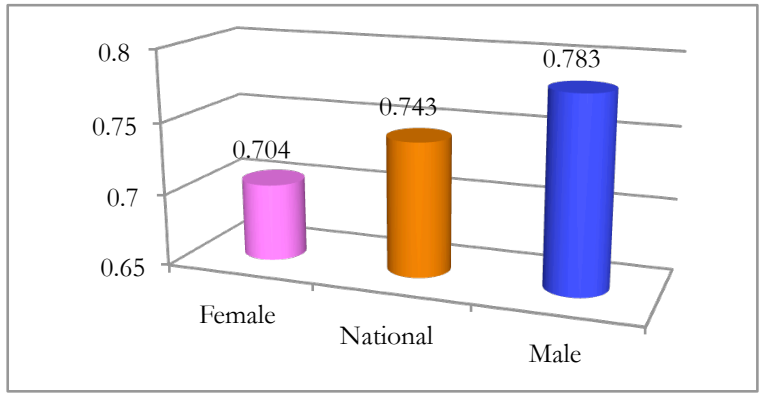

Figure 11: GNH Index by gender 


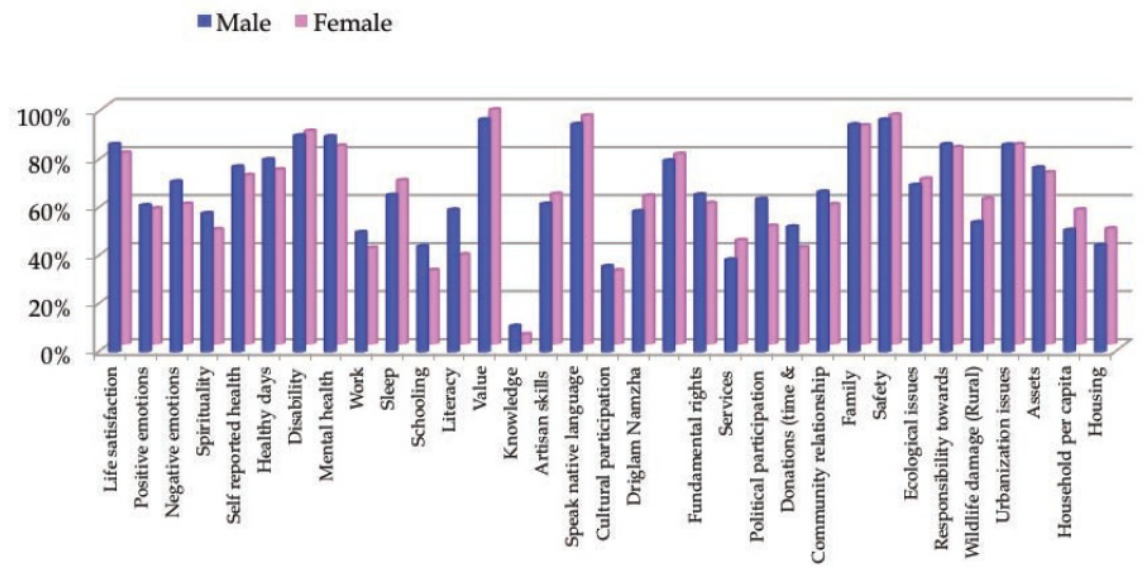

Figure 12: Percentage of Bhutanese having sufficiency in each indicator by gender

By gender we can see that men are happier than women. As table 7 shows, forty-nine per cent of men are happy, while only one-third of women are happy, a result which is both striking and statistically significant. Domain contributions show an equivalent contribution of health, time use, governance and culture, for men and women. Women do better in living standards and ecology with contributions of $10 \%$ of these two domains. Men do better in education and community vitality. 
An Extensive Analysis of GNH Index

Table 7: GNH indices and percentage of happy people by socio-demographic groups

\begin{tabular}{|c|c|c|c|c|c|c|c|c|c|c|}
\hline \multirow[b]{2}{*}{ Indicator } & \multirow[b]{2}{*}{ National } & \multicolumn{2}{|c|}{ Region } & \multicolumn{2}{|c|}{ Gender } & \multicolumn{5}{|c|}{ Marital status } \\
\hline & & Rural & Urban & Male & Female & Widowed & Separated & Divorced & Married & $\begin{array}{l}\text { Never } \\
\text { married }\end{array}$ \\
\hline GNH & 0.743 & 0.726 & 0.790 & 0.783 & 0.704 & 0.625 & 0.661 & 0.721 & 0.747 & 0.791 \\
\hline $\begin{array}{l}\text { Percentage } \\
\text { of happy people }\end{array}$ & $41 \%$ & $37 \%$ & $50 \%$ & $49 \%$ & $33 \%$ & $19 \%$ & $27 \%$ & $36 \%$ & $42 \%$ & $50 \%$ \\
\hline
\end{tabular}




\section{GNH by Age Group and Marital Status}

Happiness by age group shows a decreasing trend. The highest values of GNH correspond to people aged 22 to 29 years old. The lowest are for people aged 85 years old or more (Figure 13).

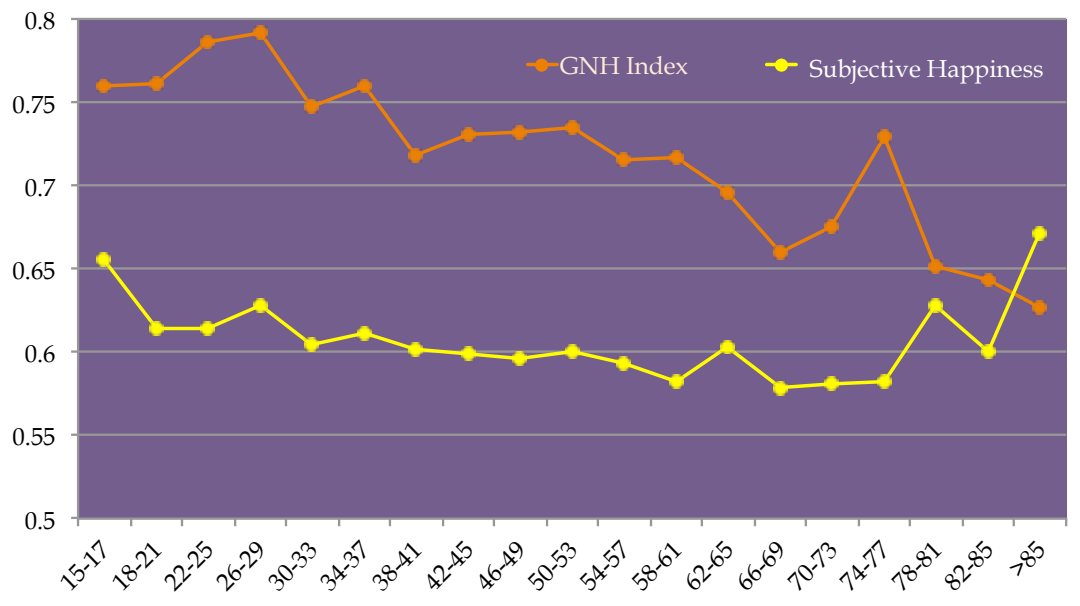

Figure 13: GNH values by age group

Table 7 also reports the GNH indices by marital status. Across the different marital status categories we see an increasing trend of both GNH Index and percentage of happy people, from left to right, this is from widowed to never married categories. Thus when compared to the national index and national percentage of happy people, only married and never married groups are above the national statistics. Widowed, separated and divorced groups are below the national benchmark.

\section{GNH by Educational Level and Occupational Status}

Figures 14 and 15, plot the values of the GNH Index by educational group and by occupational status. Note that the sample is not representative by either of these categories due to very small sample sizes in higher education and in some occupational categories such as national work force and monk/nun. So these results can only be considered as illustrative. We see that happiness is lower among those having no formal education. We also see that happiness does vary by occupation, with civil servants having higher GNH than farmers as a whole, for example. 


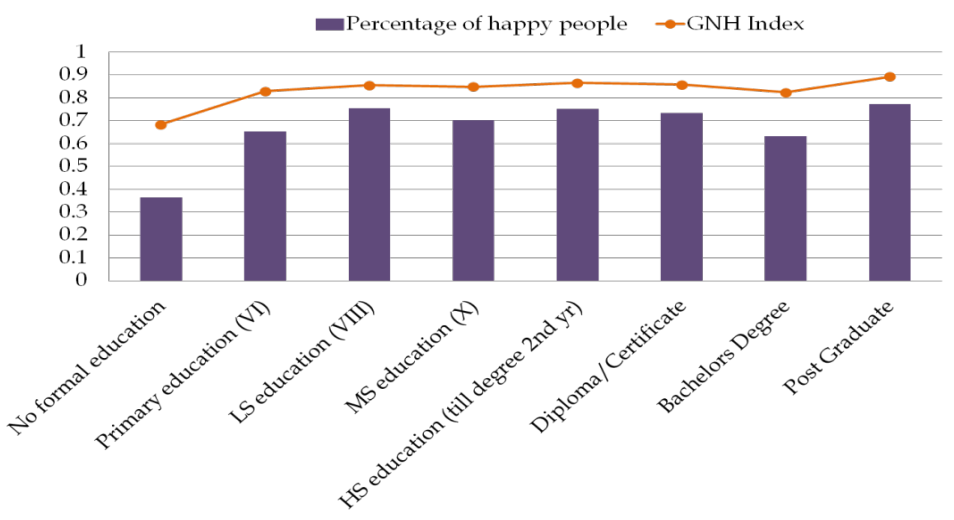

Figure 14: GNH Index and percentage of happy people by education level

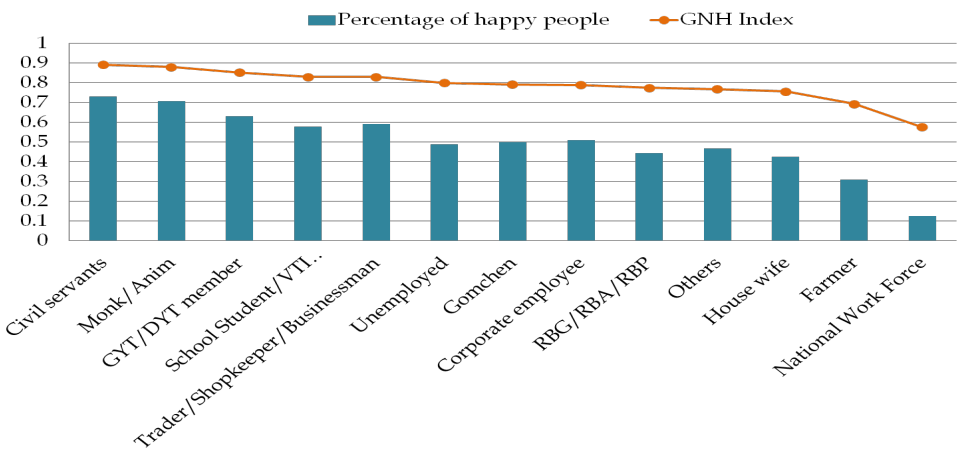

Figure 15: GNH Index and percentage of bappy people by occupational status

\section{Part III. GNH Index and Policy: Increasing Happiness}

The GNH Index is formulated to provide an incentive to increase happiness. Its aim is not only to assess the status of happy people in a society. It is also concerned with the status of not-yet-happy people. This concern for unhappiness is in line with the nation's vision of Bhutan. His Majesty Jigme Khesar Namgyel Wangchuk, $5^{\text {th }}$ King of Bhutan, clearly says that: 'the nation's Vision can only be fulfilled if the scope of our dreams and aspirations are matched by the reality of our commitment to nurturing our future citizens.'

Increasing happiness is a policy concern that involves civil servants, business leaders, and all citizens of Bhutan. The GNH Index can help them address it in practical ways. To increase happiness one needs to 
identify people who are not yet happy. Once this segment of the population is identified, one needs to know the domains in which they lack sufficiency. This two-step identification procedure provides the basis for analysis that is of direct relevance for policy.

As mentioned in the results section, the structure of the GNH Index allows us to analyse those who are not identified as happy and the dimensions or domains in which they lack sufficiency. We reflect these using the statistics for an Alkire-Foster poverty measure, remembering that the sufficiency cutoffs are set at higher levels than poverty lines and the range of domains is more extensive than might be common in poverty measures.

Table 8 presents the values of the national headcount ratio, and national breadth measure for not-yet-happy people using a threshold of $66 \%$. According to the GNH Index, $59 \%$ of Bhutanese do not fulfil the threshold of being happy in six or more than six domains; they are identified as being not-yet-happy. Of the Bhutanese who are not-yethappy, on average they have insufficiency in $43 \%$ of the domains (roughly equal to four domains).

Table 8: GNH not-yet-happy people and other measures

\begin{tabular}{|c|c|c|c|}
\hline $\begin{array}{l}2007 \text { Income } \\
\text { Poverty }\end{array}$ & $\begin{array}{l}2010 \text { Multi-dimensional } \\
\text { Poverty }\end{array}$ & \multicolumn{2}{|c|}{$\begin{array}{l}\text { GNH: 'Not -yet-happy’ } \\
\text { people }\end{array}$} \\
\hline National Monetary & National MPI & National & National \\
\hline Head Count Ratio & Head Count Ratio & Head Count Ratio & Breadth \\
\hline $23 \%$ & $26 \%$ & $59 \%$ & $43 \%$ \\
\hline
\end{tabular}

Table 8 presents the values of the national headcount ratios using the 2007 national monetary poverty line and the 2010 multidimensional poverty index. Note that the two poverty measures are measuring different underlying phenomena from GNH. Naturally happiness or well-being is a more demanding goal as well as a more well-rounded goal than poverty reduction. So it is to be expected that the figures of not-yet-happy people will be larger than the poverty headcounts. The national income poverty headcount ratio obtained with the 2007 poverty line of per capita consumption identifies $23 \%$ of the Bhutanese population as income poor. When complemented with non-income measures, the 2010 National Multidimensional Poverty Index (MPI) indicates that $26 \%$ of the Bhutanese are multidimensionally poor. The non-income domains considered in the MPI comprise health and education, while the income domain is measured by living standards instead of per capita consumption. The GNH value for not-yet-happy people extends the three-dimensional approach of the MPI to a nine-dimensional perspective, by adding psychological wellbeing, time use, cultural diversity, good governance, 
community vitality, and ecological diversity to the analysis. And it replaces the very basic cutoffs used in a poverty measure with cutoffs that reflect 'sufficiency.' Thus all of these measures are needed for public policy.

The poverty measures focus government attention on those who are suffering the most in material terms hence whose needs in some sense have a kind of priority. The GNH measure provides the overall goal for society, instead of GDP per capita. It includes all citizens, young and old, rich and poor, in key domains of their flourishing. While one will expect material poverty rates to decline - as indeed they have thus far across Bhutan - the GNH index will enable policy makers to see whether that decline in material poverty is being accompanied by a decline in other social and environmental insufficiencies - or whether perhaps cultural, social, and psychological insufficiencies may increase as material deprivation decreases. Thimphu is an interesting example of a dzongkhag in which the material achievements are very high indeed, but the corresponding social and cultural achievements are lower. Thus Thimphu's GNH value is lower than one would expect by considering either its poverty rates or its average income levels.

Clearly, happiness is deeply personal. Some of these people may regard themselves as fully flourishing. That is why we need to discuss GNH widely in Bhutan. Towards this goal, this section will first compare the insufficiencies between happy and not-yet-happy people. Then, we will analyse the structure of the insufficiencies among the not-yet-happy by domain and see which domains and indicators should be targeted in priority for increasing happiness. Finally, we will look at the insufficiencies by dzongkhag, by region, by gender, by age group, by marital status, and by occupational group of the not-yet-happy people. This analysis will provide information for policy recommendations towards increasing happiness and reducing insufficiencies.

\section{Achievements of Happy vs Not-yet-happy People}

Figure 16 below compares the achievements of happy versus unhappy people. There is considerable variation in the achievements between both groups. Across all $33 \mathrm{GNH}$ indicators we see that there is no indicator in which happy people (orange bars) have more insufficiency than not-yethappy (blue bars). But some are relatively close - such as knowledge or community. In other indicators such as services, housing, services, spirituality, and life satisfaction, happy people's achievements are, on average, markedly different from those of not-yet-happy people. 
\% insufficiency amongst the not-yet-happy \% insufficiency amongst the happy

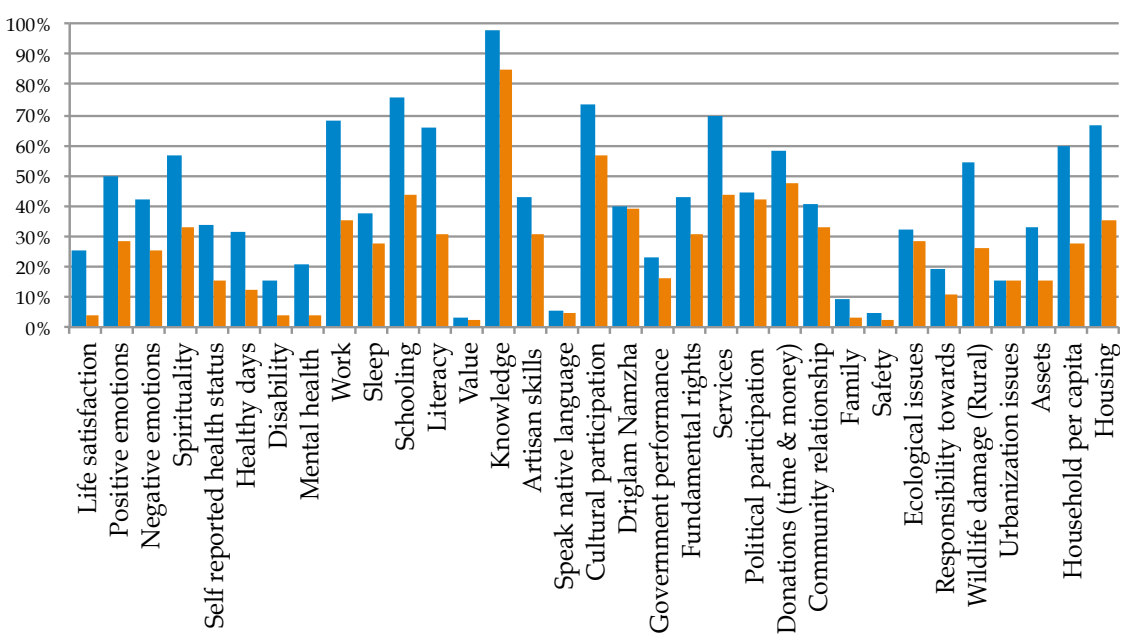

Figure 16: Comparing the percentage of people who are insufficient among the happy and the not-yet-happy

Domain indicators are from left to right: psychological wellbeing: 1-4; health: 5-8; time use: 9-10; education: 11-14; culture: 15-18; governance: 19-22; community: 23-26; ecology: 27-30; living standard: 31-33.

By domain, the not-yet-happy group always has higher insufficiency with respect to psychological wellbeing (all indicators), health (all indicators), and time use, although the groups are closest with respect to sleep. In education, culture, and governance, the groups are least different in value, language, Driglam Namzha, and political participation. Both have highest deprivations in education. In community, ecology, and living standard, the strong differences are in wildlife damage and in living standard. Happy and not-yet-happy people's insufficiencies in community and ecology are otherwise rather close and in urbanization, almost equal.

\section{Structure of the Insufficiencies among the not-yet-happy}

Table 9 presents the contribution of each of the 33 indicators and each of the nine domains composing GNH to insufficiencies among the not-yethappy, in decreasing order. This decomposition is very useful for identifying those domains that contribute the most to the insufficiency status of the not-yet-happy people. From table 9 we see that health is the lowest contributor to unhappiness $(6.1 \%)$ followed by community vitality $(7.4 \%)$. Contrastingly, education is the highest contributor to unhappiness $(15.6 \%)$. In between the maximum and minimum contributors we find 
living standard, time use and good governance contributing almost equally to unhappiness $(13.5 \%)$, followed by cultural diversity and psychological wellbeing with $11 \%$, and ecological diversity with $8.3 \%$. By indicator we see that work is the one that contributes the most to unhappiness $(8.7 \%)$, whereas value $(0.2 \%)$ is the one that contributes the least. The indicators of the time spent in work and sleep, recall, have the highest weight which is why achievements in these indicators substantially affect the GNH value.

Table 9: Contribution of indicators to unhappiness (in \%)

\begin{tabular}{llll} 
Education & $\mathbf{1 5 . 4}$ & Psychological wellbeing & $\mathbf{1 1 . 0}$ \\
Knowledge & 6.2 & Spirituality & 4.9 \\
Schooling & 4.8 & Life satisfaction & 2.2 \\
Literacy & 4.2 & Positive emotions & 2.1 \\
Value & 0.2 & Negative emotions & 1.8 \\
\hline Living standard & $\mathbf{1 3 . 6}$ & Ecological diversity & $\mathbf{8 . 3}$ \\
Housing & 5.7 & Wildlife damage & 5.5 \\
Household per capita income & 5.1 & Urbanization issues & 1.5 \\
Assets & 2.8 & Ecological issues & 0.8 \\
& & Responsibility towards environment & 0.5 \\
\hline Time use & $\mathbf{1 3 . 5}$ & Community vitality & $\mathbf{7 . 4}$ \\
Work & 8.7 & Donations & 4.5 \\
Sleep & 4.8 & Community relationship & 2.1 \\
& & Family & 0.4 \\
& & Safety & 0.4 \\
\hline Good governance & $\mathbf{1 3 . 5}$ & Health & $\mathbf{6 . 1}$ \\
Services & 7.2 & Healthy days & 2.4 \\
Political participation & 4.6 & Mental health & 1.6 \\
Fundamental rights & 1.1 & Disability & 1.2 \\
Gov. performance & 0.6 & Self-reported health status & 0.9 \\
\hline Cultural diversity & $\mathbf{1 1 . 3}$ & & \\
Cultural participation & 5.6 & & \\
Artisan skills & 3.3 & & \\
Driglam Namq $h a$ & 2.1 & & \\
Speak native language & 0.3 & & \\
\hline & & & \\
\hline
\end{tabular}

Figure 17 presents the total percentage of people who lack sufficiency in each of the 33 indicators composing the GNH. More than $50 \%$ of Bhutanese are insufficient in three of the four indicators of education; more than $40 \%$ are insufficient in two of the four indicators of good governance. This result is in line with the structure of indicator and domain contributions to unhappiness. The insufficiency in education is explained by lower levels of schooling, with $65 \%$ of the population having no formal education; literacy, with only $46 \%$ of the population being literate; and knowledge, with $37 \%$ of the population having very poor knowledge of local legends, 32\% having very poor understanding of traditional songs, and $11 \%$ having very poor understanding of local 
tshechus. Knowledge on transmission of HIV-AIDS is also quite poor with about $25 \%$ of people with knowledge of it, and $43 \%$ having some understanding. In terms of good governance, the services indicator has the strongest insufficiency, which is explained by low quality water and waste disposal services. Only $25 \%$ of the population have piped-in dwelling source of water, and $55 \%$ of them burn waste disposal. Around $37 \%$ need to walk more than 60 minutes to reach the nearest health centre, and $28 \%$ have no electricity.

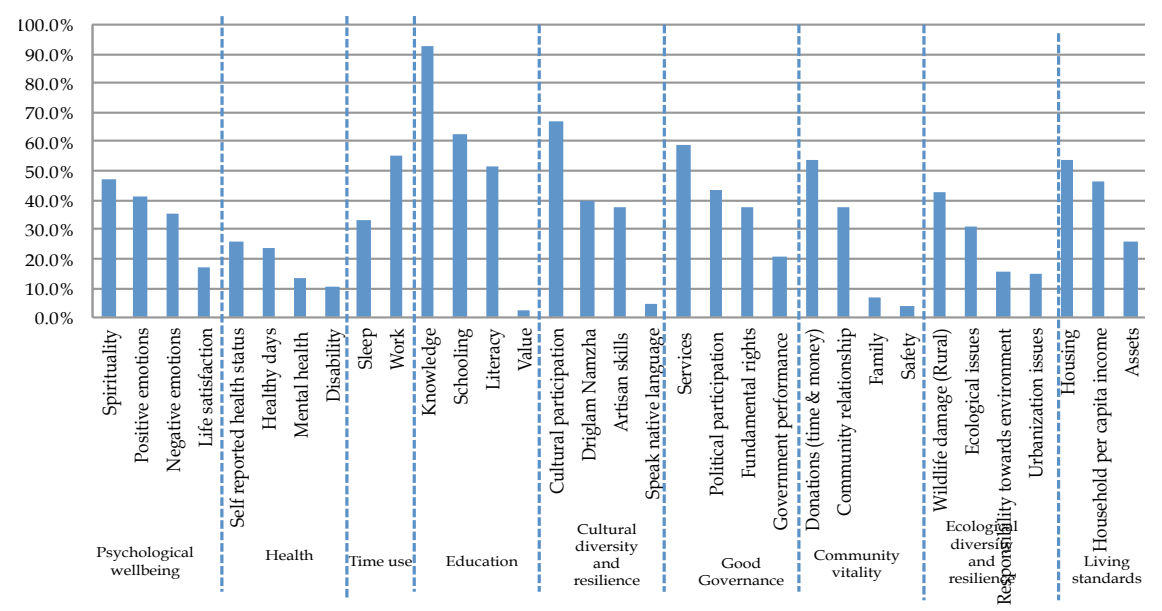

Figure 17: Total percentage of people who lack sufficiency

\section{Insufficiencies by dzongkhag and rural-urban regions}

Figure 18 presents the percentage of not-yet-happy people by dzongkhag. The unhappiness map differentiates three intervals of unhappiness that we could interpret as low (46.3-56.3\%), medium (56.31-66.25\%) and severe unhappiness (66.26-76.2\%). Thimphu and Chhukha are home to the highest number of not-yet-happy people. However these are also the districts that house the highest number of happy people. The reason for this is their population size with Thimphu having 50,000, and Chhukha 45,000 of happy Bhutanese.

By region we find more unhappy people in rural areas (63\%) compared to urban ones $(50 \%)$. However in rural areas, not-yet-happy people's average 'shortfall' (43\%) is only a little greater than the urban shortfall (41\%). This suggests less disparity in unhappiness by region than one finds in poverty measures for example. Table V-A in Appendix 6 presents the contribution of domains to unhappiness by region. Urban areas have highest insufficiency in governance, time use, and culture; whereas rural areas have highest insufficiency in education and living standards. 


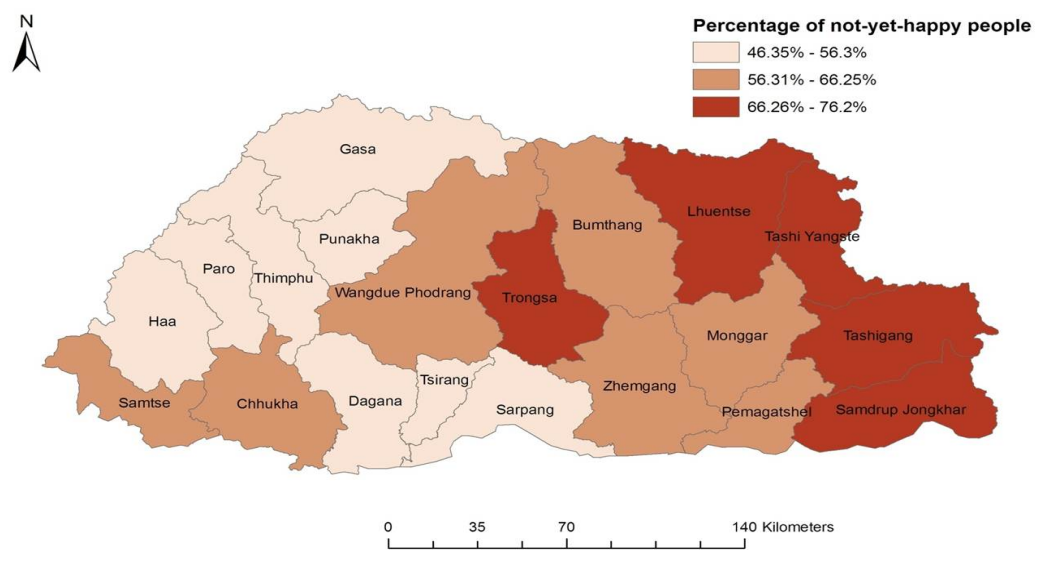

Figure 18: Percentage of not-yet-bappy people by drongkhag

\section{Insufficiencies by Gender}

Insufficiencies by gender indicate that $67 \%$ of women are not-yet-happy, and about $51 \%$ of men are not-yet-happy. Not-yet-happy people's average shortfall (lack of sufficiency) is almost the same for men (42\%) and women $(44 \%)$. The contribution to unhappiness in men and women by the respective domains is similar with education having the greatest contribution and health the smallest (Table 10).

\section{Insufficiencies by Age and Marital Status}

Happiness, as measured by GNH, varies across age groups. Young people are relatively happier than the old, although the relationship is not a perfect linear (as shown in figure 13). With respect to domain contributions young people are better educated, healthier, and have relatively good living standards. Older people do better in culture, governance, community, and psychological wellbeing. A somewhat similar trend is also observed in case of the subjective happiness. The 'happiness' question - which is not included in the GNH index - asks people to say, on a scale of 0 to 10, whether they consider themselves: 0 (Not a very happy person) -10 (Very happy person). While the percentage of those who consider their happiness to be between $7-10$ is $37 \%$, which is quite similar to the $40.8 \%$ of Bhutanese who are 'happy' by the GNH Index, the indices reflect different underlying phenomenon. Interestingly, $20.4 \%$ of Bhutanese who are extensively or deeply happy (so identified as happy by the GNH Index) report that their happiness is 7-10 on the subjective scale, but the rest - which are also $20.4 \%$ - report that their happiness is less than 7. 


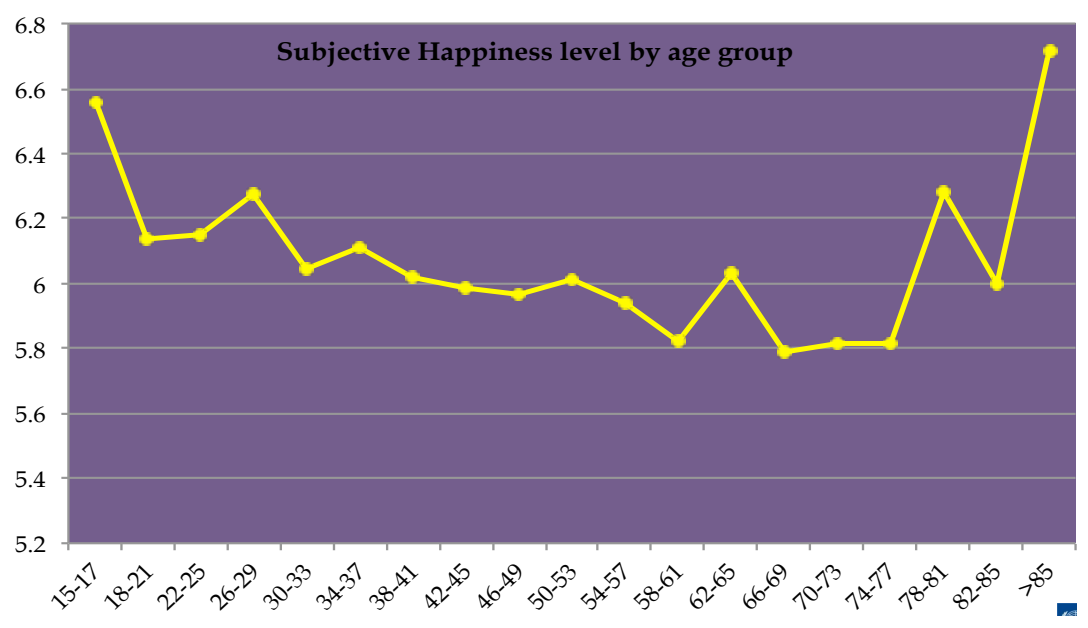

Figure 19: Subjective happiness by age group

Within psychological wellbeing, the percentage of not-yet-happy people in spirituality decreases with age. Around $40 \%$ of young people aged 20 years old or less have insufficiency in spirituality, this percentage decreases to $35 \%$ for the group of people aged between 35 to 40 years old, and to $27 \%$ for the age group of 65 years old or more. This may mean that spirituality is declining in the current generation, or it may mean that people intensify their spiritual pursuits later in life. Negative and positive emotions exhibit a less clear trend. Negative emotions decrease between the 25 or less and 35 to 40 groups of age; are stable between the group of 45 and 55 years old, and exhibit a peak for age groups 36 to 40 and 61 to 65 years old. Positive emotions are more stable between the 31 to 50 years old groups. Within health the percentage of not-yet-happy people increase with age for all indicators.

Table 10: Contribution of domains to unbappiness (in \%): by groups

\begin{tabular}{|c|c|c|c|c|c|c|c|c|c|c|}
\hline Domain & Rura & I Nationa & 1 Urban & Mal & Female & Widowed & Separate & d Divorce & d Married & $\begin{array}{l}\text { Never } \\
\text { married }\end{array}$ \\
\hline Education & 16 & 15 & 12 & $\overline{15}$ & 16 & $\overline{18}$ & 16 & 16 & 16 & 11 \\
\hline Living standards & 15 & 14 & 7 & 14 & 13 & 15 & 14 & 14 & 14 & 11 \\
\hline Time use & 13 & 14 & 15 & 14 & 13 & 11 & 11 & 13 & 14 & 12 \\
\hline Good governance & 13 & 13 & 16 & 13 & 13 & 13 & 13 & 14 & 13 & 15 \\
\hline $\begin{array}{l}\text { Psychological } \\
\text { wellbeing }\end{array}$ & 11 & 11 & 12 & 10 & 11 & 10 & 10 & 11 & 11 & 12 \\
\hline Cultural diversity & 11 & 11 & 14 & 12 & 11 & 9 & 11 & 9 & 11 & 15 \\
\hline $\begin{array}{l}\text { Ecological } \\
\text { diversity }\end{array}$ & 8 & 8 & 8 & 9 & 8 & 8 & 9 & 8 & 8 & 9 \\
\hline Health & 6 & 6 & 5 & 5 & 6 & 9 & 9 & 7 & 6 & 5 \\
\hline $\begin{array}{l}\text { Community } \\
\text { vitality }\end{array}$ & 6 & 7 & 11 & 7 & 8 & 6 & 8 & 8 & 7 & 9 \\
\hline
\end{tabular}


The percentage of unhappy people also differs by marital status. Fifty per cent of never married people are not-yet-happy, compared to $81 \%$ of widowed ones. Married, divorced and separated people have also varying percentages between $58 \%, 64 \%$ and $73 \%$, respectively. However, their average insufficiency is stable, around $40 \%$ for all marital groups. There is not a big contrast between married, divorced, separated, and widowed in what concerns insufficiencies by indicator. Widowed and Divorced enjoy a little less culture. Never married show a different profile of deprivations (table 10).

\section{Insufficiencies by Occupational Category}

As mentioned above, the analysis of insufficiencies by occupational group should be takes as illustrative only. The Bhutanese survey used for GNH computation is not representative by occupational group, and some of the occupational categories are very small, and further research should be required to verify their accuracy.

Figure 20 presents the percentage of not-yet-happy people by occupational group, as well as the average insufficiency amongst the not-yet-happy people. The highest percentage of unhappy people corresponds to the national work force occupational category ( $88 \%$ ). This is followed by $70 \%$ of farmers and $58 \%$ of house wives. The least unhappy categories are that of civil servants, with $27 \%$ of people, and monks with $29 \%$ of people. Contrastingly, the category profile of the average insufficiency is relatively equal. Civil servants have the lowest severity (0.4), while housewives have the most (nearly 0.5). The sample is not fully representative and these are not robust rankings. The national workforce is clearly and strongly the unhappiest group - they are often poorly paid, migrants doing manual labour such as taking care of roads. Clearly, it is the worst group followed by farmers, the biggest group in the survey. 


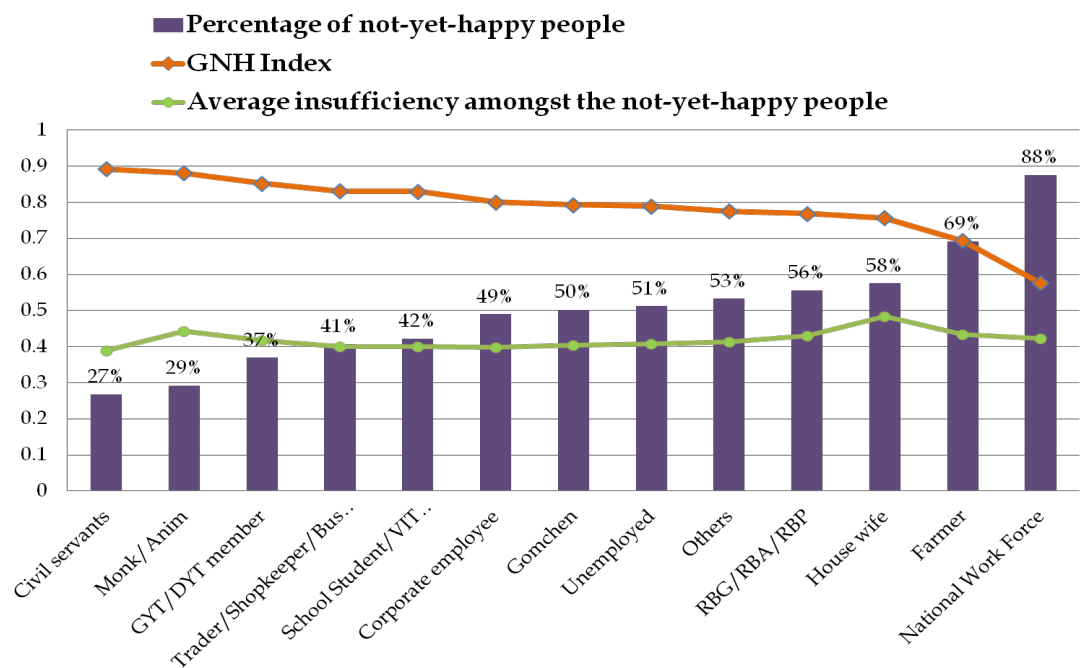

Figure 20: Head count ratio and severity by occupational group

\section{The Deeply Happy}

Any analysis of the happy people would be incomplete without a brief exploration of the subset of happy people who are identified as deeply happy. These comprise $8.3 \%$ of the population. Two-thirds of these are male, and one-third are female. Sixty-nine per cent of the deeply happy people live in rural areas and $31 \%$ in urban areas. The ages are spread from less than 20 years old to more than 65 , with $59 \%$ of the deeply happy people being less than or equal to 40 years old. Deeply happy people live in every single district of Bhutan, with the highest numbers living in Thimphu, Samtse and Chhukha. Still, only 12\% of Bhutan's deeply happy people live in Thimphu. Eighty-four per cent of the deeply happy people are married and twelve per cent are never married; the rest are divorced, separated or widowed. Twenty-six per cent of deeply happy people have no formal education; $28 \%$ have completed primary school; and some deeply happy people pertain to the remaining categories of education. Finally, deeply happy people pertain to every occupational category except the national workforce. The highest share of deeply happy people are farmers $-34 \%$ - followed by civil servants $(18 \%)$. This small snapshot of happiness across Bhutan shows that it is accessible to people of different ages, occupational categories, regions, and educational backgrounds. The fact that two-thirds of deeply happy people are men is of clear policy interest. 
Deeply happy people, on average, enjoy sufficiency in $81.5 \%$ of the domains. However it can be interesting, still, to look at the domains in which even they lack sufficiency. Interestingly, there are some insufficiencies in each domain, although these are very low in health. Overall, deeply happy people have the lowest deprivations among the four gradient groups in health, living standards, time use, and psychological wellbeing. They have the *highest* relative (not absolute) contributions from deprivations in governance and culture.

\section{The Many Faces of GNH}

The GNH Index, like the philosophy of GNH which motivates it, is very much a living experiment, seeking to convey more fully the colour and texture of people's lives than does the standard welfare measure of GNP per capita. It reflects the fact that happiness is a deeply personal matter and people will rarely agree on a set definition. Indeed, happiness has many faces, as the GNH survey shows. Here are the stories of just some happy people whose experiences of GNH were captured in the 2010 survey and who were identified as happy by the GNH Index.

These profiles help to enrich our understanding of happiness according to GNH and show that different groups - literate or illiterate, urban or rural, young or old, monk, farmer, or corporate worker - can all be happy according to these models.

One such happy person in the GNH survey was a married corporate employee aged 35 living in urban Chhukha. He has completed 10th class and has achieved sufficiency in nearly all indicators. He was a bit sleep deprived and did not feel a deep sense of belonging to his community, but was overall very satisfied with his life. When asked what contributed most to happiness he said: to be healthy, to meet basic needs, to have peace in the family, and to be religious.

Another happy person whose experiences were captured in the GNH survey was a married woman farmer aged 44 living in rural Tongsa. She was illiterate and was deprived due to wildlife damage to her crops, and thought she never felt forgiveness among the positive emotions - yet was happy. She mused that she felt happy when she was able to do her household work, when she was harvesting potatoes, and as she wove.

Another happy person in the GNH survey was a widowed gomchen aged 70 living in rural Thimphu. He had no formal education and was deprived in education, housing, sleep and did not participate politically. He observed 
that getting good agricultural products from the land contributes to happiness.

Another happy person as defined by the GNH Index is an unmarried young woman aged 26 living in urban Tashigang. She completed a bachelor's degree and is a civil servant living alone. She scores highly across domains, although she misses a sense of belonging. When asked what contributes to her happiness she replied: love, family, friends, education, and enough money.

\section{Policy Implications}

The preceding analysis suggests that to increase happiness, Bhutan needs a joint effort of its government, its community and its citizens (individuals or households). In terms of the indicators composing the $\mathrm{GNH}$, the different groups focus on an overlapping set of indicators. To give an illustrative list, the community effort must focus on donations, safety, community relationship, cultural participation, wildlife damage, and Driglam Namæha. The government policies must address political participation, services, government performance, fundamental rights, literacy, schooling, knowledge, value, Zoring Chusum skills, urban issues, ecological issues, healthy days, disability, and mental health. But equally importantly, people and households must themselves work to address life satisfaction, positive and negative emotions, self-reported health, mental health, work, sleep, responsibility towards environment, native languages, assets, housing, family, and household per capita income. This triangulation between efforts indicates that individuals are linked to each other, that communities affect one another, and so does government.

GNH is created when different groups work to do what they do best. The government and private sector should provide meaningful work, services and products. Community, civil society, and religious groups should cultivate meaningful relationships and collective action. Individuals and households should shape their own happiness by caring about sharing, relationships, and authentic self-direction and teaching their children to do likewise.

\section{Sustaining GNH}

The GNH is constructed of 33 indicators covering the nine elements of the GNH. To be fully happy, six or more domains should be fulfilled for every person. From a policy angle this means that all government projects and policies are to work together to maximize GNH in Bhutan. 
- A school advances education. But it can also help children fill other bowls. It can teach values and so fill the community 'bowl' and teach skills so as to improve the income component of the living standards bowl in the future.

- A hospital advances health. But it can also help patients to learn to meditate and thus help fill people with culture and enhance psychological wellbeing; it can have green trees and so fill ecology.

- A road contributes to living standards. But maybe the community should also talk about how they will respond with wisdom to the influences that the road will bring, so that they can keep their bowls of community vitality and culture full.

- Business managers may consider how they can not only advance living standards but also how they can offer their employees' family life, psychological wellbeing, care for ecology, and embody good governance.

Thus the key priorities are:

- Intentionally support existing GNH achievements that are valued from erosion due to cultural change.

- Incorporate GNH Index questions into more regular surveys to ensure timely detection of erosion.

- Prepare materials for different ages, region, and occupational groups of Bhutanese on how to increase GNH for oneself, with examples.

\section{Robustness Analysis - Indicators, Cutoffs, $k$, Weights.}

As the GNH Index is a new tool, we subject it to the usual robustness tests for the Alkire-Foster class of measures. These comprise the robustness of the GNH index to changes in cutoffs (k) and weights. We also developed additional robustness tests for the composition of insufficiencies. ${ }^{20}$ This is because the policy response to the GNH will be

20 This section draws upon and implements, for the first time, the new tests described in Alkire and Ballon (2012). 
determined by the composition of insufficiencies among not-yet-happy people. Thus, we wanted to ensure that the GNH Index is also robust to changes in weights and in the overall happiness cutoff. In this case, we analyse the robustness of the contributions of dzongkhags and indicators to the GNH Index for different $k$ values and weighting schemes.

The robustness analysis presented in this section is structured in three parts. In part one, we first assess the variability of the GNH Index nationally and across dzongkhags by looking at the standard errors. This analysis is needed in order to infer the precision of the GNH indices. This is followed by the sensitivity analysis of dzongkhags' ranks to changes in cutoffs $(k)$. We report the percentage of pairwise comparisons that remain robust to variations in $k$, as well as two statistics of rank correlations. The second and third parts of the robustness section present the sensitivity analysis of dzongkhags' and indicators' contributions to overall GNH, respectively. We test for robustness to cutoffs $(k)$ and weights sequentially. For this purpose we consider three alternative weighting schemes. We group the nine domains of happiness into three subgroups and allocate more weight to one subgroup at a time. The analysis in these two sections uses non-parametric tests for rank data. That is, we test the robustness of these two decompositions of overall happiness in terms of the variability of the rank distributions created by each weighting scheme, and for a range of happiness cutoffs.

\section{Robustness Ia: Standard Errors}

The first necessary calculation is that of standard errors both of the GNH Index overall and of the indices for respective dzongkhags. This is done following the standard method for Alkire-Foster measures (Yalonetzky 2010). As can be seen in Figure 8, the standard errors are relatively high. This is due in part to relatively small sample sizes at the dzongkhag level. What is also immediately apparent is that there is relatively little inequality across the dzongkhag GNH Index values. In other words, we see a surprising degree of equality across the GNH Index values. They range from just over 0.65 to just over 0.80 in value, and 17 of the 20 dzongkhags are between 0.7 and 0.8 in value. While this lack of inequality across dzongkhags is fundamentally good news, the closeness of their values combined with the magnitude of standard errors does affect subsequent robustness tests as we shall see, because the strict 'ranking' of districts is not the appropriate comparison upon which to anchor assessments of robustness. 


\section{Robustness Ib: Robustness to Changes in the Happiness (k) Cutoff}

The happiness cutoff is set such that a person who enjoys sufficiency in $66 \%$ of dimensions is considered to be happy. Correspondingly, we can see that if a person has insufficiencies in $34 \%$ or more of the dimensions, she is not considered to be happy. A first question is to what extent the selection of the $k$ cutoff - at $34 \%$ of insufficiencies (which is equivalent to $66 \%$ of sufficiencies) - affects the subsequent analysis. This is a particularly important question in the case of the GNH Index, because it is the first time that such a happiness indicator has been constructed. Hence we need to test the robustness of relevant comparisons for a range of plausible $k$ values. We do so for the value of $\mathrm{M}_{0}$. Because $\mathrm{GNH}=1-\mathrm{M}_{0}$ there will be a direct linkage between the robustness of $M_{0}$ and of the GNH Index. In what follows we refer to the $k$ cutoff as being $(100 \%-$ happiness cutoff). That is, if the happiness cutoff is $66 \%$, the $k$ cutoff is $34 \%$.

The range of plausible $k$ values clearly extends below $34 \%$. For example, in 2008 the value of $k$ was set at the value of the least-weighted indicator to create a 'union' approach in which a person who had insufficiency in any indicator, even $1 \%$ of the domains, was identified as not happy. It was not clear whether the plausible range of $k$ values extends above $34 \%$. If a person who had achieved sufficiency in five out of the nine dimensions was considered to be happy, this was widely regarded to be considered too un-demanding. For this reason we consider the plausible range of $k$ values to be between $1 \%$ and $34 \%$. To be precise, we chose four values of $k$ which have intuitive meaning and compare across them. We chose $k=$ $(34,23,12$, and 1). We also report the figures for $k=45$, but, as mentioned above, this is for academic interest only, as this higher value of $k$ would not be appropriate in practice. Intuitively, we are considering robustness across situations in which someone has achieved sufficiency in six domains $(k=34)$, seven domains $(k=23)$, eight domains $(k=12)$, or nine domains $(k=1)$, to be happy. And we also report the results for five domains $(k=45)$.

To test the robustness of the GNH Index to changes in $k$ we first consider pairwise comparisons across dzongkhags' GNH ranks, despite their very similar GNH values. The comparison of the ordinal position of districts will give insights about the change in GNH district indices in response to different cutoffs. We additionally obtain the rank correlations for the precise rankings of districts. These correlations provide an intuitive understanding of the relationship across rank distributions. Table 11 
presents the Spearman $R$ and Kendall tau $\mathrm{b}^{21}$ rank correlation coefficients for the selected $k$ values. The Spearman rank correlations are always 0.97 and above for the aforementioned values of $k$ and fall to 0.92 if we consider $k=45$. The Kendall tau $\mathrm{b}$ rank correlations are 0.87 and higher, and fall to 0.79 for $k=45$. Table 12 reports the percentage of pairwise comparisons that are robust to $k$ - those that do not vary with changes in $k$. There is, as expected, a great deal of noise in the precise rankings: only $55 \%$ of pairwise comparisons are robust ( $45 \%$ if $k=45$ ); however, given the positive situation of equality combined with the small sample size and thus large standard errors on these variables, comparisons of pairwise rankings do not seem appropriate, so we move on to consider grouped rankings.

To avoid the false precision of pairwise comparisons we group the districts into four categories by their ranked GNH $\left(1-\mathrm{M}_{0}\right)$ values, with the first one below the $25^{\text {th }}$ percentile, the second below the median, the third below the $75^{\text {th }}$ percentile and the last up to 100 (table 13a). As is evident from table $13 \mathrm{~b}$ below, between $k=1$ and $k=34$, only two districts switch categories: Punakha and Thimphu, both of which switch between the top and second categories.22 We can conclude that the grouping of dzongkhags into four categories is robust to changes in the happiness cutoff (table 13b).

Table 11: Rank Correlations

\begin{tabular}{|c|c|c|c|c|}
\hline \multicolumn{5}{|c|}{ Spearman $R$ rank correlations for $\mathbf{M}_{0}$} \\
\hline k value & $\begin{array}{c}k=1 \\
2\end{array}$ & $\begin{array}{c}k=2 \\
3\end{array}$ & $\begin{array}{c}k=3 \\
4\end{array}$ & $\begin{array}{c}k=4 \\
5\end{array}$ \\
\hline$k=1$ & 0.995 & 0.985 & 0.982 & $\begin{array}{r}0 . \\
949\end{array}$ \\
\hline$k=12$ & & 0.982 & 0.976 & 0.958 \\
\hline$k=23$ & & & 0.968 & 0.916 \\
\hline$k=34$ & & & & 0.95 \\
\hline
\end{tabular}

\begin{tabular}{lcccc}
\multicolumn{5}{c}{ Kendall $\operatorname{tau}$ b rank correlations for $\mathbf{M}_{0}$} \\
$k$ value & $k=1$ & $k=2$ & $k=3$ & $k=4$ \\
& 2 & 3 & 4 & 5 \\
\hline$k=1$ & \multirow{2}{*}{0.968} & 0.926 & 0.926 & 0.863 \\
$k=12$ & & 0.916 & 0.895 & 0.874 \\
$k=23$ & & & 0.874 & 0.789 \\
$k=34$ & & & & 0.874 \\
\hline
\end{tabular}

\footnotetext{
${ }^{21}$ Although Kendall tau and Spearman R are comparable in terms of their statistical power, they usually differ in magnitude. More importantly their interpretations are also different. Spearman $R$ can be thought of as the regular Pearson product-moment correlation coefficient as computed from ranks. Kendall tau rather represents a probability. Specifically, it is the difference between the probability that the GNH Index of the districts leads to the same order for two different $k$ values versus the probability that the GNH district indices are in different orders for the same two $k$ values. Three different variants of tau are computed $(a, b$, and $c)$, these differ with regard as to how tied ranks are handled. In most cases these values will be fairly similar (Kendall, 1948, 1975; Everitt, 1977; Siegel and Castellan, 1988).

22 If we also include $k=45$, then four additional districts switch categories: Mongar, Pemagatshel, Tashigang and Zhemgang
}

68 
Table 12: Percentage of pairwise comparisons that do not vary to changes in $k$

\begin{tabular}{lrrrr}
\multicolumn{1}{c}{$k$ value } & $k=12$ & $k=23$ & $k=34$ & $k=45$ \\
\hline$k=1$ & $70 \%$ & $55 \%$ & $50 \%$ & $40 \%$ \\
$k=12$ & & $50 \%$ & $50 \%$ & $40 \%$ \\
$k=23$ & & & $55 \%$ & $40 \%$ \\
$k=34$ & & & & $45 \%$ \\
\hline
\end{tabular}

Table 13a: Categories of GNH

\begin{tabular}{llll}
\hline Top GNH & Upper GNH & Lower GNH & Lowest GNH \\
\hline Dagana & Chhukha & Bumthang & Lhuntse \\
Haa & Gasa & Mongar & Samdrup Jongkhar \\
Paro & Punakha & Pemagatshel & Tashiyangtse \\
Sarpang & Tsirang & Samtse & Tashigang \\
Thimphu & Zhemgang & Wangdue Phodrang & Trongsa \\
\hline
\end{tabular}

Table 13b: Robustness to ke by category

\begin{tabular}{lrrrr}
\multicolumn{1}{c}{ District } & $\mathrm{k}=1$ & $\mathrm{k}=12$ & $\mathrm{k}=23$ & $\mathrm{k}=34$ \\
\hline Bumthang & 2 & 2 & 2 & 2 \\
Chhukha & 1 & 1 & 1 & 1 \\
Dagana & 0 & 0 & 0 & 0 \\
Gasa & 0 & 0 & 1 & 1 \\
Haa & 0 & 0 & 0 & 0 \\
Lhuntse & 3 & 3 & 3 & 3 \\
Mongar & 2 & 2 & 2 & 2 \\
Paro & 0 & 0 & 0 & 0 \\
Pemagatshel & 2 & 2 & 2 & 2 \\
Punakha & 1 & 1 & 0 & 1 \\
Samdrup Jongkhar & 3 & 3 & 3 & 3 \\
Samtse & 2 & 2 & 2 & 2 \\
Sarpang & 0 & 0 & 0 & 0 \\
Tashiyangtse & 3 & 3 & 3 & 3 \\
Tashigang & 3 & 3 & 3 & 3 \\
Thimphu & 1 & 1 & 1 & 0 \\
Trongsa & 3 & 3 & 3 & 3 \\
Tsirang & 1 & 1 & 1 & 1 \\
Wangdue Phodrang & 2 & 2 & 2 & 2 \\
Zhemgang & 1 & 1 & 1 & 1 \\
\hline Categores are: & & & &
\end{tabular}

Categories are:

0 - less than $25^{\text {th }}$ percentile

1 - 25th percentile to median

2 - Median to 75 th percentile

3 - above 75 th percentile 


\section{Robustness II: Robustness of Drongkhags' Contributions to Overall Happiness by Cutoff and Weights}

To understand the changes in dzongkhags' contributions to overall happiness we recall that $\mathrm{M}_{0}$ depends on the matrix of indicator achievements of the population $(X)$, the set of weights $(w)$, and the overall cutoff $(k)$ (which is $100 \%$ minus the happiness cutoff). ${ }^{23}$ As was explained in the methodological section, $\mathrm{M}_{0}$ can be decomposed by subgroup or dzongkhag ( () , this is:

$$
M_{0}(X, w, k)=\sum_{l=1}^{m} \frac{n_{l}}{n} M_{0}\left(X_{l}, w, k\right), l=1, \ldots, m
$$

where: $M_{0}\left(X_{l}, w, k\right)$ is the (l) dzongkhag's $\mathrm{M}_{0}$ index, and $\frac{n_{l}}{n}$ is the Dzongkhag's population share.

Thus to test for robustness one can consider the following two alternatives. We can test for the sensitivity of the GNH or $M_{0}$ values to changes in the weights allocated to each indicator for a given cutoff value. Alternatively, we can test for robustness of GNH to changes in the cutoff value for a given weighting scheme. In the first part of this robustness section we have already tested the robustness of the GNH indices to different $k$ values given a weighting structure where dimensional weights receive 33\% each (see below). In this second part of the robustness section we combine these two alternatives. Before presenting the results we formalise the changes in dzongkhags' contributions to overall happiness.

A change in the weighting scheme from $w$ to $w_{a}$ given the cutoff $(k)$ could be formalised as follows:

$$
\Delta M_{0}\left(w, w_{a} \mid X, k\right)=M_{0}\left(w_{a} \mid X, k\right)-M_{0}(w \mid X, k)
$$

where: $\Delta M_{0}\left(w, w_{a} \mid X, k\right)$ denotes the change in $M_{0}$.

As $M_{0}$ could be decomposed by subgroup (dzongkhag), the change in $M_{0}$ leads to:

$$
\Delta M_{0}\left(w, w_{a} \mid X, k\right)=\sum_{l=1}^{m} \frac{n_{l}}{n} \Delta M_{0}\left(w, w_{a} \mid X_{l}, k\right), l=1, \ldots, m
$$

${ }^{23} M_{0}$ is also a function of the vector of sufficiency cut-offs $₹$.

70 
Similarly, a change in the cutoff from $k$ to $k_{a}$ for a given weighting scheme (w) could be formalised as follows:

$$
\Delta M_{0}\left(k, k_{a} \mid X, w\right)=\sum_{l=1}^{m} \frac{n_{l}}{n} \Delta M_{0}\left(k, k_{a} \mid X_{l}, w\right), l=1, \ldots, m
$$

Thus the change in $M_{0}$ equals the sum of the weighted subgroup changes in $M_{0}$ (either with respect to the weight or the cutoff), where subgroup weights are given by the population shares. This provides an intuitive interpretation of the change in $M_{0}$ resulting from a change in the weighting scheme or the cutoff, as resulting from the change in the dzongkhags' contributions to overall GNH or $M_{0}$.

To explore the robustness of dzongkhags' contributions to overall happiness we look at the changes in GNH categories (table 13a) which occur as a result of changes in the indicators' weights. We consider a baseline scenario and three alternative weighting schemes. For this purpose, we group the nine domains of happiness into three subgroups. The first subgroup includes psychological wellbeing, cultural diversity and resilience, and community vitality. The second includes time use, good governance, ecological diversity and resilience. The third group includes living standards, health, and education. Our baseline scenario takes the weights that have been presented in the preceding sections - that is, a value of $33 \%$ to each subgroup. The alternative weighting structures assign half of the weight to one subgroup and a quarter of the weight to the other two subgroups (table 14). This unequal dimensional weighting scheme keeps the indicators' weights within each domain unchanged. The first weighting scheme (Case 1) assigns half of the overall weight to the third subgroup of dimensions comprising living standards, health and education. The second and third subgroups receive a quarter of the overall weight, that is, $25 \%$ each. Case 2 redistributes the weights and assigns $50 \%$ of the weight to time use, good governance, ecological diversity and resilience. The first and third subgroups receive $25 \%$ each. Lastly, Case 3 allocates $50 \%$ of the overall weight to the first subgroup of domains and leaves the remaining two quarters to subgroups 2 and 3 .

Table 15 reports the changes in $\mathrm{M}_{0}$ and, by implication, $\mathrm{GNH}$ by categories, and for different $k$ values. We observe that the more robust scenario is given by Case 2 (compared to the baseline). A shift from the baseline weighting scheme of $33 \%$ to a dimensional group weighting of $25-50-25 \%$ leads to very few changes in districts' rank categories. More than half of the districts (11 out of 20) do not change their category for any $k$ value. In two out of five of the $k$ values used for testing robustness 
Chhukha, Gasa, and Thimphu gain one rank category, while Punakha loses one rank category in the new ranking given by Case2. ${ }^{24}$ Also for Case 2 Pemagatshel gains one positional category for $k=23$ and $k=34$, and loses one positional category for $k=45$.

Case 1 versus baseline provides an intermediate scenario in terms of variability of the district rankings by category according to $M_{0}$. Allocating greater weight to time use, good governance, and ecological diversity and resilience leads to more frequent shifts in district ranks by category. With Case 1 ten districts do not change rank categories. Trongsa shifts one category for all $k$ values. In four out of the five $k$ values considered, Pemagatshel and Zhemgang gain one category, shifting towards a higher rank category, while Samtse loses one category thus is positioned in a lower rank category.

Table 14: Alternative weighting schemes

\begin{tabular}{|c|c|c|c|c|c|}
\hline \multicolumn{2}{|c|}{ Domain - Group } & Baseline & $\begin{array}{c}\text { Case 1: } \\
25-25- \\
50\end{array}$ & $\begin{array}{l}\text { Case 2: } \\
25-50- \\
25\end{array}$ & $\begin{array}{l}\text { Case 3: } \\
50-25- \\
25\end{array}$ \\
\hline Group 1 & $\begin{array}{l}\text { Psychological } \\
\text { wellbeing } \\
\text { Cultural diversity } \\
\text { \& resilience } \\
\text { Community } \\
\text { vitality }\end{array}$ & $33 \%$ & $25 \%$ & $25 \%$ & $50 \%$ \\
\hline Group 2 & $\begin{array}{l}\text { Time use } \\
\text { Good governance } \\
\text { Ecological } \\
\text { diversity \& } \\
\text { resilience }\end{array}$ & $33 \%$ & $25 \%$ & $50 \%$ & $25 \%$ \\
\hline Group 3 & $\begin{array}{l}\text { Living Standard } \\
\text { Health } \\
\text { Education }\end{array}$ & $33 \%$ & $50 \%$ & $25 \%$ & $25 \%$ \\
\hline
\end{tabular}

Case 3 versus baseline turns out to be the most volatile scenario when it comes to district rankings by category (table 15). Assigning greater weight to psychological wellbeing, cultural diversity and community vitality shows much more sensitivity in category rankings of Thimphu, Tashigang, and Chhukha. Thimphu shifts up to three rank categories with $k=34$. More precisely, from being below the $25^{\text {th }}$ percentile, when weights are of $33 \%$, Thimphu ranks above the $75^{\text {th }}$ percentile when greater weight is given to domains of Group 1. Put simply, Thimphu's GNH Index is much lower if

\footnotetext{
24 A positive difference indicates that district loses a position, shifting to a low rank category. A negative difference thus indicates a gain in the district's rank category.
} 
these categories are given a higher weighting. For $k=45$, Thimphu shifts two rank categories upwards and one category upwards for all remaining $k$ values. Tashigang shifts two positions downwards for $k=1,12,23$, and 34 . From being in the $75^{\text {th }}$ percentile or above (in baseline), this district ranks in the $25^{\text {th }}$ percentile according to Case 3 . For $k=45$ Tashigang loses one rank category. Chhukha gains two rank categories for $k$ values above 23, and one category for $k$ values below 23 . Tashiyangtse and Tsirang shift positions for all $k$ values, while Wangdue Phodrang and Bumthang change positions in four out of five $k$ values.

To deepen our analysis of the robustness of dzongkhags' contributions to overall happiness, we examine whether the weighting structure has an effect on the dzongkhags' contributions to overall happiness. For this purpose we use three non-parametric tests that are applied for testing differences among distributions. Our null hypothesis states that, for a given cutoff, the distributions of the dzongkhags' contributions to overall happiness do not differ across weighting schemes. Table 16 reports the Friedman test and the Kendall's W test by $k$ value. ${ }^{25}$ For all cutoffs both tests are not significant at the $5 \%$ level, as shown by the $\chi 2$ statistic and the associated $p$-value which is greater than $5 \%$. Based on these results we can conclude that the dzongkhags' contributions to overall happiness do not (statistically) differ with changes in the weighting structure for all $k$ values considered. This indicates that the dzongkhags' contributions to overall happiness are statistically robust to changes in weights.

\footnotetext{
25 The Friedman test is applicable to problems with repeated-measures designs, in our case the dzongkhags' contributions to overall happiness by (repeated) weighting scheme. The Kendall's coefficient of concordance (W) is a measure of the strength of the relationship among distributions. The coefficient of concordance ranges from 0 to 1 , with higher values denoting a stronger difference across distributions.
} 
Karma Ura, Sabina Alkire, Tshoki Zangmo \& Karma Wangdi

Table 15: Changes in dzongkhag GNH categories for different weights and $k$ values

\begin{tabular}{|c|c|c|c|c|c|c|c|c|c|c|c|c|c|c|c|}
\hline \multirow[b]{2}{*}{ District } & \multicolumn{5}{|c|}{ Baseline - Case 1} & \multicolumn{5}{|c|}{ Baseline - Case 2} & \multicolumn{5}{|c|}{ Baseline - Case 3} \\
\hline & $\mathrm{k}=1$ & $\mathrm{k}=12$ & $\mathrm{k}=23$ & $\mathrm{k}=34$ & $\mathrm{k}=45$ & $\mathrm{k}=1$ & $\mathrm{k}=12$ & $\mathrm{k}=23$ & $\mathrm{k}=34$ & $\mathrm{k}=45$ & $\mathrm{k}=1$ & $\mathrm{k}=12$ & $\mathrm{k}=23$ & $\mathrm{k}=34$ & $\mathrm{k}=45$ \\
\hline Bumthang & 0 & 0 & 0 & 1 & 0 & 0 & 0 & 1 & 0 & -1 & -1 & -1 & 0 & -1 & -1 \\
\hline Chhukha & 0 & 0 & 0 & 1 & 0 & 0 & 0 & -1 & -1 & 0 & -1 & -1 & -2 & -2 & -2 \\
\hline Dagana & 0 & 0 & 0 & -1 & 0 & 0 & 0 & 0 & 0 & 0 & 0 & 0 & 0 & -1 & 0 \\
\hline Haa & 0 & 0 & 0 & 0 & 0 & 0 & 0 & 0 & 0 & 0 & -1 & -1 & -1 & -1 & 0 \\
\hline Lhuntse & 0 & 0 & 0 & 0 & 0 & 0 & 0 & 1 & 0 & 0 & 0 & 0 & 0 & 1 & 1 \\
\hline Mongar & 0 & 0 & 0 & 0 & -1 & 0 & 0 & 0 & 0 & -1 & 1 & 1 & 1 & 1 & 0 \\
\hline Paro & 0 & 0 & 0 & 0 & 0 & 0 & 0 & 0 & 0 & 0 & 0 & 0 & 0 & 0 & 0 \\
\hline Samdrup Jongkhar & 0 & 0 & 0 & 0 & 0 & 0 & 0 & 0 & 0 & 0 & 0 & 0 & 0 & 0 & 0 \\
\hline Samtse & 1 & 1 & 1 & 0 & 1 & 0 & 0 & 0 & 1 & 0 & 0 & 0 & 0 & 0 & 0 \\
\hline Sarpang & 0 & 0 & 0 & 0 & 0 & 0 & 0 & 0 & 0 & 0 & 0 & 0 & 0 & 0 & 0 \\
\hline Tashiyangtse & 0 & 0 & 0 & 0 & 0 & 0 & 0 & 0 & 0 & 0 & 1 & 1 & 1 & 1 & 2 \\
\hline Tashigang & 0 & 0 & 0 & 0 & -1 & 0 & 0 & 0 & 1 & 0 & 2 & 2 & 2 & 2 & 1 \\
\hline Thimphu & 1 & 1 & 1 & 0 & 0 & 0 & 0 & 0 & -1 & -1 & -1 & -1 & -1 & -3 & -2 \\
\hline
\end{tabular}

Note: A positive difference indicates that a district loses a position, shifting to a lower rank category. A negative difference indicates a gain in the district's rank. 


\section{Robustness III: Robustness of Indicators' Contributions to Overall Happiness by Cutoff and Weighting Scheme}

The above tests for robustness focused on the ranking of dzongkhags initially by using pairwise comparisons and then by the four 'groups'. However, to increase GNH, policymakers will want to analyse the profile of insufficiencies in each dzongkhag and use these to guide investments and interventions. Because of their policy relevance, it is vital to know whether the composition of insufficiencies is also highly sensitive to the weights used. Naturally, there will be some sensitivity - after all, the weights directly affect the composition profiles of each dzongkhag. But in this section we ask whether the policy responses would vary fundamentally across the same weighting structure as used in the previous sections.

To test for robustness of indicators' contribution to the GNH index by $k$ value and weighting scheme we recall that $M_{0}$ can be broken down by indicator, after identification, as follows:

$$
M_{0}(X, w, k, z)=\sum_{j=1}^{d} w_{j} h_{j}(X, w, k, z)
$$

where: $h_{j}(X, w, k, z)$ and $w_{j}$ are, respectively, the censored headcount and weight attached to the $j$-th indicator.

Developing the above expression we obtain:

$$
M_{0}(X, w, k, z)=\frac{1}{n} \sum_{i=1}^{n}\left[\sum_{j=1}^{d} w_{j} I\left[x_{i j}<z_{j}\right]\right] I\left[c_{i}(w) \geq k\right]
$$

where: $I[$.$] is the indicator function that takes the value of one if the$ condition is satisfied. In our case, $I\left[x_{i j}<z_{j}\right]$ will take the value of one for all those individuals $(i)$ who are below the insufficiency cutoff $z$ in the $j$-th indicator $\left(z_{j}\right)$; and $I\left[c_{i} \geq k\right]$ will take the value of one for all those individuals who are identified as not yet happy. We should note that $c_{i}$ depends on the set of weights $c_{i}(w)$.

Thus the preceding formula clearly also shows the possible sources of change in $M_{0}$. This is either a change in the insufficiency cutoff $z$, or in the set of weights $w$, or in the happiness cutoff $k$. As noted it also shows that changes in the set of weights $w$ will lead to joint changes in the weights themselves and in the censored deprivation scores $c_{i}$ at the same time. 
Our robustness analysis of indicators' contributions to overall GNH does not consider changes in the insufficiency cutoffs $z$. We focus on changes in either the set of weights $w$ or in the cutoff $k$ that is associated with the happiness cutoff.

Additionally the percentage contribution of each indicator (j) to overall happiness $\left(C_{j}\right)$ is:

$$
C_{j}(X, w, k, z)=\frac{w_{j} h_{j}(X, w, k, z)}{\sum_{j=1}^{d} w_{j} h_{j}(X, w, k, z)}=\frac{w_{j} h_{j}(X, w, k, z)}{M_{0}}
$$

which gives an appealing interpretation of $M_{0}$ as the weighted sum of the censored headcounts.

Taking these elements into account, a change in the weighting scheme from $w$ to $w_{a}$ given the cutoff $(k)$ could be formalised as follows:

$$
\Delta M_{0}\left(w, w_{a} \mid X, k, z\right)=M_{0}\left(w_{a} \mid X, k, z\right)-M_{0}(w \mid X, k, z)
$$

which in terms of the indicator breakdown leads to:

$$
\begin{aligned}
\Delta M_{0}\left(w, w_{a} \mid X, k, z\right) & \\
& =\frac{1}{n} \sum_{i=1}^{n} \sum_{j=1}^{d} I\left[x_{i j}<z_{j}\right] \quad[\underbrace{w_{a, j} I\left[c_{i}\left(w_{a}\right) \geq k\right]}_{f\left(w_{a}\right)} \\
& -\underbrace{w_{j} I\left[c_{i}(w) \geq k\right]}_{f(w)}]
\end{aligned}
$$

The second right-hand-side expression in brackets allow us to visualise the interdependent effect of the set of weights and the censored insufficiency scores resulting from changes in the set of weights. Hence, when we test for robustness in the indicators' contributions to overall happiness, due to a change in the weighting scheme from $w$ to $w_{a}$, the change in $M_{0}$ is given by the joint change in the weight (from $w$ to $w_{a}$ ) and in the censored headcount.

A change in the cutoff from $k$ to $k_{a}$ for a given weighting scheme ( $\left.w\right)$ does not show such interdependence and could be simply expressed as a weighted change (due to $k$ ) of the censored headcounts as follows: 


$$
\Delta M_{0}\left(k, k_{a} \mid X, w, z\right)=\Delta\left[\sum_{j=1}^{d} w_{j} h_{j}\left(k, k_{a} \mid X, w, z\right)\right]
$$

Table 16: Dzongkhags' contribution to $M_{0}$ by weighting scheme and $k$ value

\begin{tabular}{|c|c|c|c|c|c|c|}
\hline \multicolumn{7}{|c|}{ Non parametric tests } \\
\hline \multicolumn{2}{|c|}{ Test Statistics } & $k=1$ & $\mathrm{k}=12$ & $\mathrm{k}=23$ & $\mathrm{k}=34$ & $\mathrm{k}=45$ \\
\hline Friedman Test & Chi-Square & 4.560 & 5.400 & 5.220 & 5.580 & 1.500 \\
\hline $\begin{array}{l}\text { Kendall's coeff. } \\
\text { Concordance }\end{array}$ & W & .076 & .090 & .087 & .093 & .025 \\
\hline Degrees of freedom & & 3 & 3 & 3 & 3 & 3 \\
\hline Asymp. Significance & & 207 & .145 & .156 & .134 & .682 \\
\hline
\end{tabular}

Table 17 presents the indicators' contribution to $M_{0}$ by weighting scheme when $k=34$. The sixth column of this table reports the average contribution of each indicator across the four weighting sets. For example, we see that the average contribution of service is $7.29 \% .^{26}$ The seventh column of table 7 shows the variance of the contribution of each indicator also across the four possible set of weights. These two statistics provide initial insights about the sensitivity of the contributions to changes in weights. However, as the contributions of the indicators vary in magnitude, in table 18 we compare the coefficient of variation of each indicator's contribution across the four sets of weights, and for the different $k$ values. The coefficient of variation is a normalised measure of dispersion that allow us to compare the varying indicators' contributions more easily. From this table we observe that across $k$ values the coefficient of variation is more or less stable.

Table 17: Indicators' contributions to $M_{0}$ by weighting scheme for $k=34$

\begin{tabular}{lcccccc} 
Indicator & Baseline & $\begin{array}{c}\text { Case 1: } \\
\text { 25-25- }\end{array}$ & $\begin{array}{c}\mathbf{2 :} \\
\mathbf{2 5 - 5 0 -}\end{array}$ & $\begin{array}{c}\text { Case } \\
\mathbf{5 0 - 2 5 -}\end{array}$ & Mean & Variance \\
& & $\mathbf{5 0}$ & $\mathbf{2 5}$ & $\mathbf{2 5}$ & & \\
\hline Life satisfaction & 2.26 & 1.58 & 1.54 & 3.62 & 2.25 & 0.94 \\
Positive emotions & 2.01 & 1.37 & 1.40 & 3.23 & 2.00 & 0.76 \\
Negative emotions & 1.82 & 1.24 & 1.28 & 2.91 & 1.81 & 0.61 \\
Spirituality & 4.78 & 3.27 & 3.34 & 7.78 & 4.79 & 4.44 \\
Self rep. health & 0.87 & 1.26 & 0.61 & 0.66 & 0.85 & 0.09 \\
Healthy days & 2.42 & 3.62 & 1.68 & 1.80 & 2.38 & 0.79 \\
Disability & 1.17 & 1.78 & 0.80 & 0.87 & 1.15 & 0.20 \\
Mental health & 1.60 & 2.37 & 1.09 & 1.24 & 1.57 & 0.33 \\
& & & & & &
\end{tabular}

26 This is: $(7.37+5.32+11.10+5.37) / 4$. 


\begin{tabular}{|c|c|c|c|c|c|c|}
\hline Work & 8.79 & 6.13 & 13.66 & 6.32 & 8.72 & 12.29 \\
\hline Sleep & 4.55 & 3.01 & 7.37 & 3.28 & 4.55 & 3.98 \\
\hline Schooling & 5.02 & 9.49 & 4.38 & 4.37 & 5.82 & 6.09 \\
\hline Literacy & 4.46 & 8.46 & 3.83 & 3.91 & 5.16 & 4.91 \\
\hline Value & 0.17 & 0.16 & 0.09 & 0.11 & 0.13 & 0.00 \\
\hline Knowledge & 6.24 & 7.26 & 3.65 & 3.74 & 5.22 & 3.28 \\
\hline Artisan skills & 3.44 & 2.36 & 2.44 & 5.58 & 3.45 & 2.25 \\
\hline \multicolumn{7}{|l|}{ Speak native } \\
\hline language & 0.24 & 0.15 & 0.17 & 0.42 & 0.24 & 0.01 \\
\hline \multicolumn{7}{|l|}{ Cultural } \\
\hline participation & 5.60 & 3.85 & 3.99 & 9.03 & 5.62 & 5.80 \\
\hline Driglam Namzba & 2.06 & 1.36 & 1.50 & 3.36 & 2.07 & 0.83 \\
\hline \multicolumn{7}{|l|}{ Government } \\
\hline performance & 0.61 & 0.41 & 0.91 & 0.48 & 0.60 & 0.05 \\
\hline Fundamental rights & 1.07 & 0.76 & 1.60 & 0.81 & 1.06 & 0.15 \\
\hline Service & 7.37 & 5.32 & 11.10 & 5.37 & 7.29 & 7.37 \\
\hline \multicolumn{7}{|l|}{ Political } \\
\hline participation & 4.03 & 2.67 & 6.16 & 3.29 & 4.04 & 2.31 \\
\hline Donations & 4.31 & 2.94 & 3.10 & 7.11 & 4.36 & 3.72 \\
\hline \multicolumn{7}{|l|}{ Community } \\
\hline relationship & 1.91 & 1.22 & 1.36 & 3.13 & 1.90 & 0.76 \\
\hline Family & 0.53 & 0.36 & 0.36 & 0.85 & 0.53 & 0.05 \\
\hline Safety & 0.34 & 0.22 & 0.23 & 0.56 & 0.34 & 0.03 \\
\hline Ecological issues & 0.79 & 0.55 & 1.19 & 0.59 & 0.78 & 0.09 \\
\hline Resp. environment & 0.52 & 0.36 & 0.75 & 0.39 & 0.51 & 0.03 \\
\hline Wildlife damage & 5.98 & 4.42 & 8.90 & 4.24 & 5.88 & 4.67 \\
\hline Urbanization issues & 1.09 & 0.64 & 1.70 & 0.85 & 1.07 & 0.21 \\
\hline Assets & 2.86 & 4.33 & 1.97 & 2.10 & 2.81 & 1.18 \\
\hline \multicolumn{7}{|l|}{ Household p.c } \\
\hline income & 5.09 & 7.80 & 3.59 & 3.66 & 5.04 & 3.88 \\
\hline \multirow[t]{2}{*}{ Housing } & 6.02 & 9.29 & 4.26 & 4.34 & 5.98 & 5.53 \\
\hline & 100.0 & 100.0 & 100.0 & 100.0 & 100.0 & \\
\hline
\end{tabular}

In order to concretise our assessment of the robustness of the indicators' contributions to overall GNH (or $M_{0}$ ) we conduct a Friedman test to evaluate the differences of these contributions across weights (table 19). The test is significant at 5\% level as all p-values are below the critical threshold, which shows that the composition of insufficiencies is robust to changes in weights. The Kendall's coefficient of concordance is greater than 0.10 which indicates that there is only a weak difference among the four sets of weights. To grasp which weighting structure or structures cause the composition to diverge the most, we conduct a Wilcoxon test for each pair of weighting structures. Table 20 reports the comparison of ranks for each pair of sets of weights. For example, when we compare the baseline set of weights with those of Case 1 we observe that for $k=34$ there are 23 negative ranks and 10 positive ranks. A negative rank indicates that the contribution of the indicator is ranked higher (is cardinally bigger) with the set of weights of the baseline scenario, compared to Case 1. A positive rank indicates the opposite. This information is used in the computation of the Wilcoxon Test reported in table 21. The Wilcoxon test 
statistics indicate that the null hypothesis of equality of the distribution of the indicators' contributions across pair of weighting schemes cannot be rejected at 5\% level, for all $k$ values. Therefore we can conclude that the indicators' contributions to $M_{0}$ are robust across the four sets of weights and the chosen $k$ values.

Table 18: Indicators' contribution to $M_{0}$ by weighting scheme and $k$ value

\section{Coefficient of variation}

\begin{tabular}{|c|c|c|c|c|c|}
\hline Indicator & $k=1$ & $k=12$ & $k=23$ & $k=34$ & $k=45$ \\
\hline Life satisfaction & 0.38 & 0.37 & 0.38 & 0.43 & 0.49 \\
\hline Positive emotions & 0.38 & 0.38 & 0.39 & 0.43 & 0.46 \\
\hline Negative emotions & 0.38 & 0.38 & 0.39 & 0.43 & 0.45 \\
\hline Spirituality & 0.37 & 0.37 & 0.39 & 0.44 & 0.47 \\
\hline Self rep. health & 0.34 & 0.34 & 0.34 & 0.35 & 0.37 \\
\hline Healthy days & 0.34 & 0.34 & 0.35 & 0.37 & 0.39 \\
\hline Disability & 0.34 & 0.34 & 0.35 & 0.39 & 0.42 \\
\hline Mental health & 0.34 & 0.34 & 0.35 & 0.36 & 0.39 \\
\hline Work & 0.34 & 0.34 & 0.36 & 0.40 & 0.44 \\
\hline Sleep & 0.34 & 0.34 & 0.37 & 0.44 & 0.54 \\
\hline Schooling & 0.36 & 0.36 & 0.39 & 0.42 & 0.43 \\
\hline Literacy & 0.36 & 0.36 & 0.39 & 0.43 & 0.44 \\
\hline Value & 0.35 & 0.35 & 0.31 & 0.28 & 0.23 \\
\hline Knowledge & 0.35 & 0.35 & 0.35 & 0.35 & 0.35 \\
\hline Artisan skills & 0.37 & 0.37 & 0.39 & 0.43 & 0.45 \\
\hline Speak native language & 0.38 & 0.38 & 0.38 & 0.50 & 0.64 \\
\hline Cultural participation & 0.37 & 0.38 & 0.39 & 0.43 & 0.43 \\
\hline Driglam Namzba & 0.37 & 0.37 & 0.39 & 0.44 & 0.48 \\
\hline Government performance & 0.34 & 0.34 & 0.35 & 0.37 & 0.37 \\
\hline Fundamental rights & 0.34 & 0.34 & 0.34 & 0.36 & 0.39 \\
\hline Service & 0.34 & 0.34 & 0.35 & 0.37 & 0.39 \\
\hline Political participation & 0.34 & 0.34 & 0.36 & 0.38 & 0.43 \\
\hline Donations & 0.37 & 0.37 & 0.40 & 0.44 & 0.47 \\
\hline Community relationship & 0.37 & 0.37 & 0.39 & 0.46 & 0.50 \\
\hline Family & 0.37 & 0.38 & 0.39 & 0.44 & 0.53 \\
\hline Safety & 0.37 & 0.37 & 0.38 & 0.47 & 0.54 \\
\hline Ecological issues & 0.34 & 0.34 & 0.36 & 0.38 & 0.43 \\
\hline Resp. environment & 0.34 & 0.35 & 0.34 & 0.35 & 0.38 \\
\hline Wildlife damage & 0.34 & 0.34 & 0.34 & 0.37 & 0.39 \\
\hline Urbanization issues & 0.33 & 0.33 & 0.35 & 0.43 & 0.53 \\
\hline Assets & 0.34 & 0.34 & 0.35 & 0.39 & 0.42 \\
\hline Household p.c income & 0.33 & 0.34 & 0.35 & 0.39 & 0.42 \\
\hline Housing & 0.34 & 0.34 & 0.35 & 0.39 & 0.41 \\
\hline
\end{tabular}

Table 19: Indicators' contribution to $M_{0}$ by weighting scheme and $k$ value

\begin{tabular}{lc}
\multicolumn{2}{c}{ Test Statistics } \\
\hline Friedman Test & Chi-Square \\
Kendall's coeff. & $\mathrm{W}$ \\
Concordance & \\
Degrees of freedom & \\
Asymp. Significance & \\
\hline
\end{tabular}

\begin{tabular}{rrrrr}
\multicolumn{5}{c}{ Non-parametric tests } \\
$\mathbf{k}=\mathbf{1}$ & $\mathbf{k}=\mathbf{1 2}$ & $\mathbf{k}=\mathbf{2 3}$ & $\mathbf{k}=\mathbf{3 4}$ & $\mathbf{k}=\mathbf{4 5}$ \\
\hline 11.109 & 11.436 & 11.291 & 10.731 & 10.055 \\
.112 & .116 & .114 & .108 & .102 \\
& & & & \\
3 & 3 & 3 & 3 & 3 \\
.011 & .010 & .010 & .013 & .018 \\
\hline
\end{tabular}


Table 20: Indicators' contribution to $M_{0}$ by weighting scheme and $k$ value

\section{Weighting}

schemes

Case 1 -

Baseline

Case 2 -

Baseline

Baseline

Case1

Case 3 -

Case 1

Case 3 -

Case 2

\begin{tabular}{l} 
Cases \\
\hline Negative \\
Ranks (a) \\
Positive \\
Ranks (b) \\
Ties (c) \\
Negative \\
Ranks (a) \\
Positive \\
Ranks (b) \\
Ties (c) \\
Negative \\
Ranks (a) \\
Positive \\
Ranks (b) \\
Ties (c) \\
Negative \\
Ranks (d) \\
Positive \\
Ranks (e ) \\
Ties (f) \\
Negative \\
Ranks (d) \\
Positive \\
Ranks (e ) \\
Ties (f) \\
Negative \\
Ranks (d) \\
Positive \\
Ranks (e ) \\
Ties (f) \\
Rat \\
Tan
\end{tabular}

a. Case $j<$ Baseline, $j=1,2,3$

b. Case $\mathrm{j}>$ Baseline, $\mathrm{j}=1,2,3$

c. Case $\mathrm{j}=$ Baseline, $\mathrm{j}=1,2,3$

d. Case $\mathrm{j}+1<$ Case $\mathbf{j}, \quad \mathrm{j}=1,2,3$

e. Case $\mathrm{j}+1>$ Case $\mathrm{j}, \mathrm{j}=1,2,3$

f. Case $j+1=$ Case $j, j=1,2,3$

\section{Comparison of Ranks}

\begin{tabular}{|c|c|c|c|c|}
\hline$k=1$ & $k=12$ & $k=23$ & $k=34$ & $\mathrm{k}=45$ \\
\hline 22 & 22 & 22 & 23 & 23 \\
\hline 11 & 11 & 11 & 10 & 10 \\
\hline 0 & 0 & 0 & 0 & 0 \\
\hline 23 & 23 & 23 & 23 & 23 \\
\hline 10 & 10 & 10 & 10 & 10 \\
\hline 0 & 0 & 0 & 0 & 0 \\
\hline 21 & 21 & 21 & 21 & 21 \\
\hline 12 & 12 & 12 & 12 & 12 \\
\hline 0 & 0 & 0 & 0 & 0 \\
\hline 19 & 20 & 14 & 11 & 14 \\
\hline 14 & 13 & 19 & 22 & 19 \\
\hline 0 & 0 & 0 & 0 & 0 \\
\hline 12 & 12 & 11 & 11 & 11 \\
\hline 21 & 21 & 22 & 17 & 22 \\
\hline 0 & 0 & 0 & 5 & 0 \\
\hline 10 & 10 & 10 & 11 & 14 \\
\hline 23 & 23 & 23 & 21 & 19 \\
\hline 0 & 0 & 0 & 1 & 0 \\
\hline
\end{tabular}


Table 21: Indicators' contributions to $M_{0}$ by weighting scheme and $k$ value Wilcoxon test

\begin{tabular}{cccccc} 
Weighting & \multicolumn{7}{c}{ Wilcoxon Signed Ranks Test $-Z$} & \\
schemes & $\mathbf{k}=\mathbf{1}$ & $\mathbf{k}=\mathbf{1 2}$ & $\mathbf{k}=\mathbf{2 3}$ & $\mathbf{k}=\mathbf{3 4}$ & $\mathbf{k}=\mathbf{4 5}$ \\
\hline Case 1 - Baseline & -0.884 & $-.884^{\mathrm{b}}$ & $-.920^{\mathrm{b}}$ & -1.099 & $-.867^{\mathrm{b}}$ \\
& .376 & .376 & .357 & .272 & .386 \\
Case 2 - Baseline & -1.295 & $-1.295^{\mathrm{b}}$ & $-1.313^{\mathrm{b}}$ & -1.42 & $-1.420^{\mathrm{b}}$ \\
& .195 & .195 & .189 & .155 & .155 \\
Case 3 -Baseline & -0.777 & $-.777^{\mathrm{b}}$ & $-.706^{\mathrm{b}}$ & -0.813 & $-.420^{\mathrm{b}}$ \\
& .437 & .437 & .480 & .416 & .675 \\
Case 2 - Case1 & -0.706 & $-.706^{\mathrm{b}}$ & $-.295^{\mathrm{c}}$ & -0.688 & $-.384^{\mathrm{c}}$ \\
& .480 & .480 & .768 & .491 & .701 \\
Case 3 - Case 1 & -0.563 & $-.563^{\mathrm{c}}$ & $-.688^{\mathrm{c}}$ & -0.273 & $-.581 \mathrm{c}$ \\
& .574 & .574 & .492 & .785 & .561 \\
Case 3 - Case 2 & -0.867 & $-.867 \mathrm{c}$ & $-.867^{\mathrm{c}}$ & -0.636 & $-.384^{\mathrm{c}}$ \\
& .386 & .386 & .386 & .525 & .701 \\
\hline
\end{tabular}




\section{The Way Forward: GNH as a Policy Tool}

Aside from deepening our understanding of happiness, the GNH Index is formulated to provide an incentive to increase happiness. Civil servants, business leaders, and citizens of Bhutan may ask, 'how can I help to increase GNH?' The GNH Index can help them answer this question in practical ways. It also enables the government and others to track changes over time. In general, there are two mechanisms by which public policy action can be directed so as to increase GNH: it can either increase percentage of people who are happy or increase the percentage of domains in which not-yet-happy people enjoy sufficiency.

\section{Insufficiencies by indicator}

To improve GNH we can look at people who are not yet happy and look at the areas where they lack sufficiency $-59 \%$ of Bhutanese are not-yethappy and they are deprived in roughly four domains each. The not-yethappy people are more deprived in all 33 indicators than the happy people (figure 21). The biggest deprivations are in education, living standards and time use. Among the not-yet-happy, women are unhappier than men.

Rural people are less happy than urban people although their intensities are similar. But the composition of insufficiencies vary. The urban groups have bigger insufficiencies in governance, time and culture, and in rural areas the biggest problems are education and living standards. The difference here is thus in terms of the more material domains versus those that are about community, culture and spirituality. In Thimphu, the capital, for example, the biggest deprivations are in community vitality.

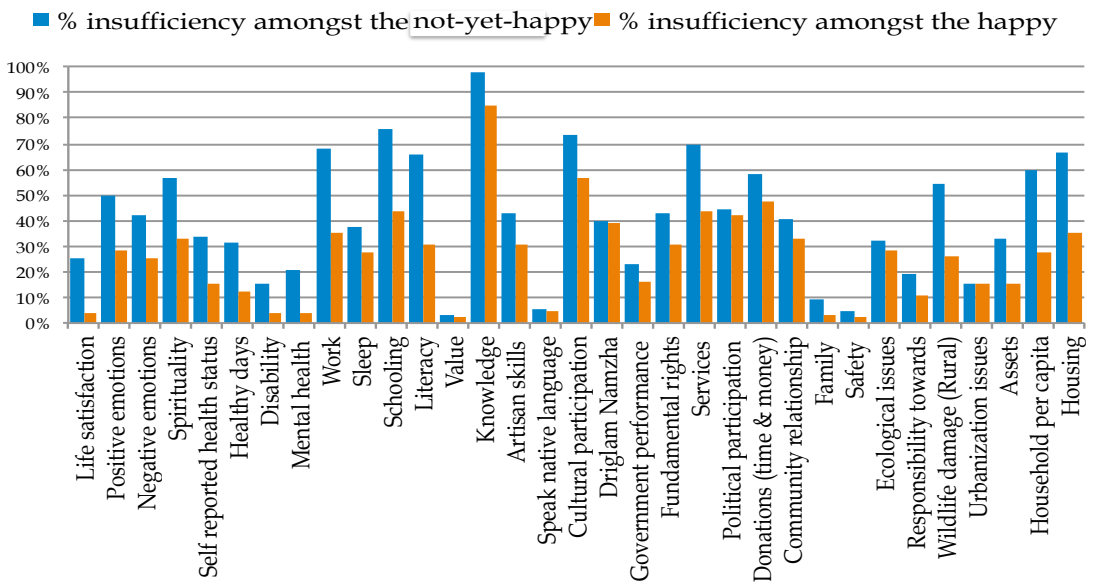

Figure 21: Proportion of people with insufficiencies in each indicator by happiness 
Across all indicators we see that there is no indicator in which orange bars are higher than blue - none in which happy people have more insufficiency than not-yet-happy. Looking at psychological wellbeing, health, and time use, we see that not-yet-happy people always have higher insufficiency. In education, culture, and governance, the groups are least different in value, language, Driglam Namzha, and political participation. Both have the highest deprivations in education. In community, ecology, and living standard, the strong differences are in wildlife damage and in living standard indicators. Happy and not-yet-happy people's insufficiencies in community and ecology are otherwise rather close and in urbanization, almost equal.

Percentage contribution to unhappiness (in \%)

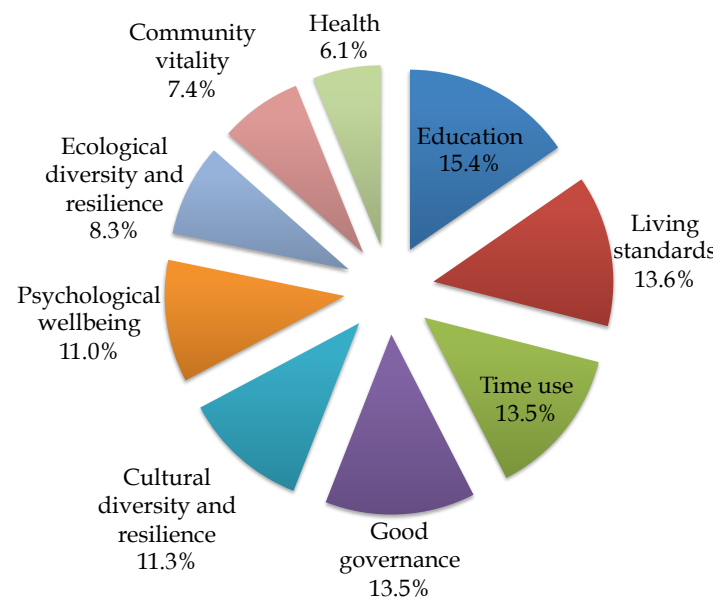

Figure 22: Contribution to unbappiness

Health is the lowest contributor to unhappiness followed by community vitality. Education is the highest contributor to unhappiness. We can also break apart each domain to see where the biggest sources of unhappiness are coming from among the indicators.

Figure 23 illustrates this for the education domain. The highest insufficiency is in the knowledge indicator. Bhutanese experience low levels of knowledge in cultural and historical aspects of the country and in health and politics. 


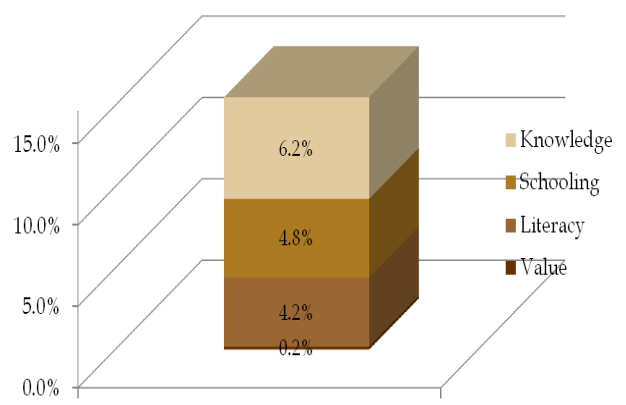

Figure 23: Contribution of Education indicators to unhappiness

\section{Who can Increase GNH?}

Increasing happiness is not only the business of government. The GNH requires civil servants, business leaders, and average citizens to ask how they can increase GNH. So in that sense, the GNH index could be offered as a public good that will provide information to the many different institutions that are seeking to improve GNH. His Majesty the King Jigme Khesar Namgyel Wangchuck clearly states that:

Our nation's vision can only be fulfilled if the scope of our dreams and aspirations are matched by the reality of our commitment to nurturing our future citizens.

The people who are not-yet-happy are an important policy priority and thus it is important to look at the areas in which they enjoy sufficiency and the areas in which they still lack sufficiency. Government, monasteries, communities and individuals and households efforts can contribute to increasing $\mathrm{GNH}$. 


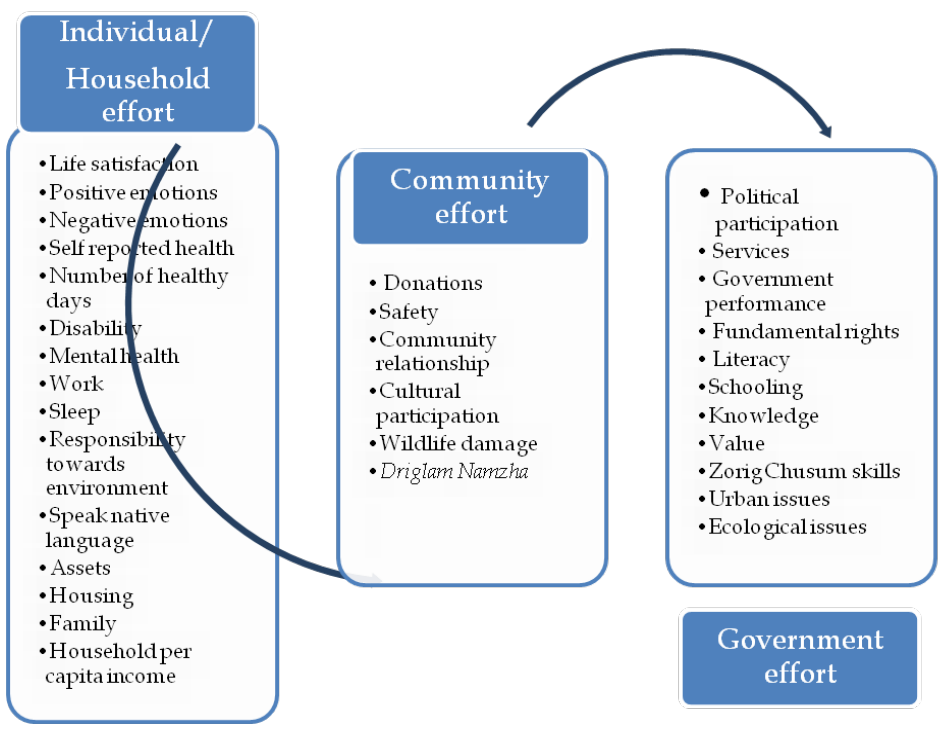

Figure 24: Overlapping responsibilities for increasing happiness

While responsibility for some indicators is shared across government, community and households, there is a lot of overlap between the areas of actions.

\section{Insufficiencies among bappiness groups}

Figure 25 shows the per cent contribution of each domain to the insufficiency of the four population groups that we identified. As can be seen, clearly the average insufficiency is lowest, as we would expect, among the deeply happy group. We can also see that the absolute contribution of each indicator is the lowest in the deeply happy group. The biggest contributions to insufficiency among the unhappy are living standards, education, and psychological wellbeing - a combination of traditional and innovative measures of wellbeing. Time pressures and a lack of governance including access to services are also very high. Deprivations in community and ecology contribute relatively less to insufficiencies of those who are not-yet-happy. 


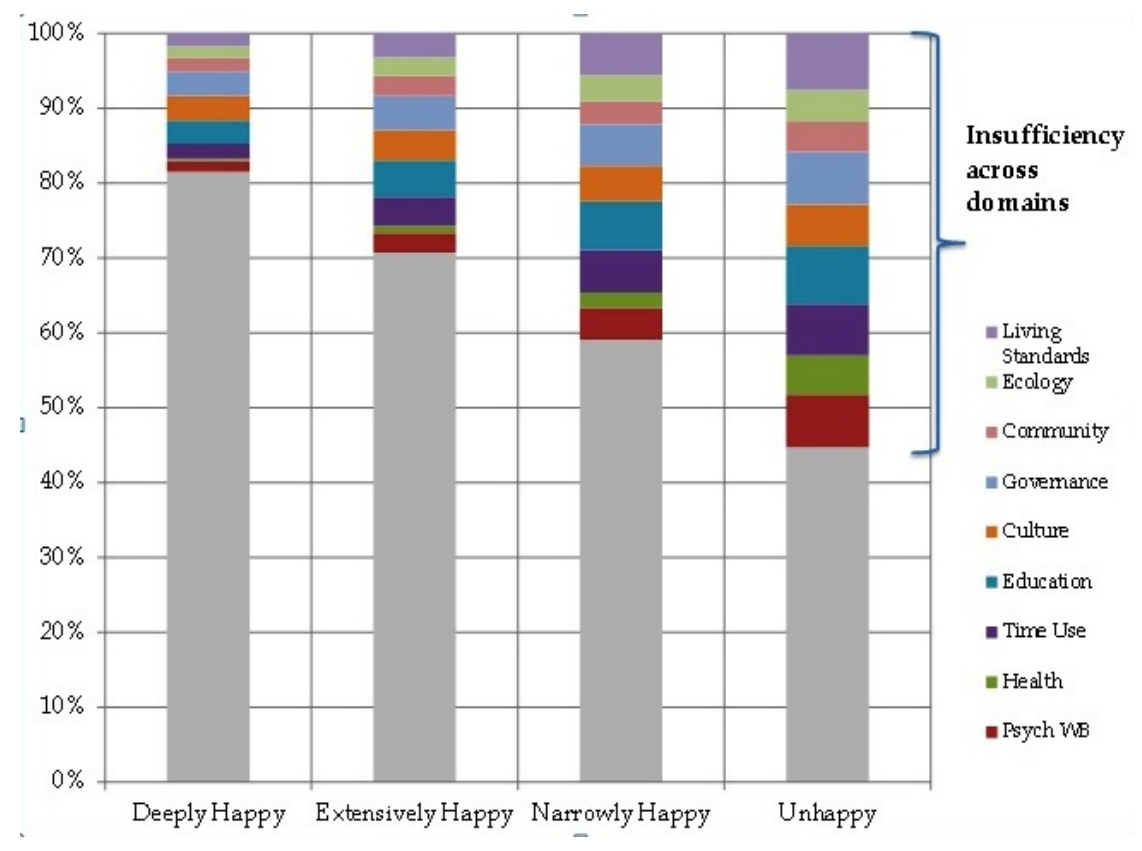

Figure 25: Insufficiencies across domains by happiness groups

\section{The unbappy}

Those who achieve sufficiency in less than half of domains are considered unhappy. In 2010, 10.4\% of Bhutanese were unhappy. Who are these people? Sixty-nine per cent of the unhappy people are women and $31 \%$ are men. Eighty-four per cent of unhappy people live in rural areas. Although the unhappy come from every age cohort, $57 \%$ of the unhappy are over 40 years old. Samtse, Tashigang, and Chhukha are home to the most unhappy people, followed by Thimphu and Samdrup Jonkhar but there are some in each district nationally. And $76 \%$ of unhappy people are married. While $90 \%$ of unhappy people have no formal education, others are found in every other educational category except that there are zero unhappy people who have completed a diploma or post-graduate studies. Seventy-nine per cent of unhappy people are farmers, but unhappy people are drawn from all occupations except that there are zero unhappy people among the monks, anim, Gewog Yargye Tshogchung (GYT) and Drongkhag Yargye Tshogchung (DYT).

Across domains, the unhappy people show markedly higher contributions to their deprivations from living standards, health deprivations, and psychological ill-being. This profile of unhappiness, when contrasted with the profile of deeply happy people, is quite striking in showing that no 
single category finds happiness unattainable. In the same way, very few categories leave one immune from unhappiness, with the possible exception of post-graduate education and the monastic or spiritually committed life. ${ }^{27}$

\section{Building GNH}

The GNH has been presented to provincial district-level leaders to allow them to review their policies against the district-level results and see how they could alter policies according to the results. The wider goal is to promote a public dialogue around the index so people can share their own understandings and appreciate how they could increase their own GNH. Policy and programme screening tools have already been in use based on the 2008 index and all agencies whether public or private are encouraged to think holistically.

${ }^{27}$ Recall that sample sizes are such that the decompositions by occupational group and higher education cannot be taken to be representative but are shared for illustrative purposes only. 


\section{Cited References}

Afsa, Cédric; Blanchet, Didier; Marcus, Vincent; Pionnier, Pierre-Alain; Rioux, Laurence; Mira d'Ercole, Marco; Ranuzzi, Giulia; Schreyer, Paul. (2008). 'Survey of Existing Approaches to Measuring SocioEconomic Progress', Paris: Commission on the Measurement of Economic Performance and social Progress (April) [available at http://www.stiglitz-senfitoussi.fr/documents/Survey_of_Existing_Approaches_to_Measu ring_Socio-Economic_Progress.pdf accessed on 14 September 2011].

Ahlbrandt, Roger S. and Cunningham, James V. (1979). A New Public Policy for Neighborhood Preservation. New York: Praeger.

Albritton, Robert B. and Bureekul, Thawilwadee. (2009). 'Are Democracy and "Good Governance" Always Compatible? Competing Values in the Thai Political Arena', Santiago: International Political Science Association - Asian Barometer Project Office, (Working Paper Series: No. 47) [available at http://www.asianbarometer.org/newenglish/publications/workin gpapers/no.47.pdf accessed on 10 October 2011].

Alkire, Sabina. (2002a). 'Dimensions of Human Development'. World Development, 30(2), 181-205.

Alkire, Sabina. (2002b). Valuing freedoms. Sen's Capability Approach and Poverty Reduction. New York, Oxford: Oxford University Press.

Alkire, Sabina. (2008). 'Choosing Dimensions: The Capability Approach and Multidimensional Poverty’. In N. Kakwani \& J. Silber (Eds.), The Many Dimensions of Poverty (pp. 89-119). New York: Palgrave Macmillan.

Alkire, Sabina. (2009). The Capability Approach to the Quality of Life. Background Paper for the Sarkozy Commission, www.stiglitz-senfitoussi.fr.

Alkire, Sabina and Ballon, Paola, 'OPHI Technical Note on Robustness to Compositions of Poverty using the AF Measure'. Research in progress.

Alkire, Sabina, and Foster, James. (2007). 'Counting and Multidimensional Poverty Measures’. OPHI working paper series, 7.

Alkire, Sabina, and Foster, James. (2011a). 'Counting and Multidimensional Poverty Measurement'. Journal of Public Economics, 95(7-8), 476-487. 
Alkire, Sabina, and Foster, James. (2011b). 'Understandings and Misunderstandings of Multidimensional Poverty Measurement'. Journal of Economic Inequality, 9(2), 289-314.

Alkire, Sabina, and Santos, Maria Emma. (2010). 'Acute Multidimensional Poverty: A New Index for Developing Countries’. OPHI working paper series, 38 .

Andrews, Rhys, Boyne, George, and Walker, Richard. (2006). 'Workforce Diversity in the Public Sector: An Evaluation of the Performance of English Local Authorities', Policy and Politics 34(2), 287-306.

Aris, Michael and Hutt, Michael. (1994) Bhutan: Aspects of Culture and Development. Gartmor: Kiscadale.

Arthaud-Day, M. L., Rode, C., Mooney, H., and Near, J. (2005). 'The Subjective Wellbeing Construct: A Test of its Convergent, Discriminant and Factorial Validity', Social Indicators Research, 74, 445-476.

Bachrach, K. and Zautra, A. J. (1985). 'Coping with a Community Stressor: The threat of a hazardous waste facility', Journal of Health and Social Behavior, 26.

Baker, Michael; Stabile, Mark and Deri, Catherine. (2003). 'What do Selfreported, Objective Measures of Health Measure?', Journal of Human Resources, 39(4),1067-1093.

Baker, D.W., Williams M.V., Parker, R.M., Gazmararian J.A. \& Nurss, J. (1999). 'Development of a brief test to measure functional health literacy.' Patient Education \& Counseling 38, 33-42.

Bardasi, Elena and Wodon, Quentin. (2009). 'Working Long Hours and Having No Choice. Time Poverty in Guinea', Washington, D. C.: The World Bank.

Basu, Kaushik. (2003). Prelude to Political Economy : a Study of the Social and Political Foundations of Economics. New York: Oxford University Press.

Beach, Betty A. (1987). 'Time Use in Rural Home-Working Families', Family Relations, 36(4), 412-416.

Belcher, Anne R., Dettmore, Diane and Holzemer, Stephen Paul. (1989). 'Spirituality and Sense of Well-Being in Persons With AIDS', Holistic Nursing Practice, 3(4), 16-25.

Bergner, M.; Bobbit, R. A.; Carter, W. B. and Gilson, B. S. (1981). 'The Sickness Impact Profile: development and final revision of a health status measure', Medical Care, 19, 787-805. 
Blanchflower, David G. and Oswald, Andrew J. (2011). 'International Happiness', National Bureau of Economic Research (NBER Working Paper Series, 16668). [available at http://www.nber.org/papers/w16668 accessed on 9 September 2011].

Bohen, Halcyone and Viveros-Long, Anamaria. (1981). Balancing Jobs and Family Life: Do Flexible Work Schedules Help? Philadelphia, PA.: Temple University Press.

Boyne, George A. and Law, Jennifer (1991). 'Accountability and Local Authority Annual Reports: The Case of Welsh District Councils', Financial Accountability and Management, 7, 179-194.

Bradburn, Norman M. (1969). The Structure of Psychological Wellbeing. Chicago: Aldine Publishing.

Brazier, J. E.; Jones, N. and Kind, P. (1993). 'Testing the Validity of the Euroqol and Comparing it with the SF-36 Health Questionnaire', Quality of Life Research, 2, 169-180.

Brighouse, Harry, \& Robeyns, Ingrid. (2010). Measuring Justice : Primary Goods and Capabilities. Cambridge; New York: Cambridge University Press.

Bruni, Luigino , Comim, Flavio , \& Pugno, Maurizio. (2008). Capabilities and Happiness. New York: Oxford University Press.

Bruni, Luigino and Porta, Pier Luigi (Eds.) (2007). Handbook on the Economics of Happiness. Cheltenham: Edward Elgar.

Burchardt, Tania. (2008). 'Time and Income Poverty', London: London School of Economics, November (Centre for the Analysis of Social Exclusion Report, 57).

Campbell, David, E. and Putnam, Robert, G . (2012). American Grace: How Religion Divides and Unites Us. New York: Simon \& Schuster.

Canadian International Development Agency (CIDA) (1996). 'Policy for CIDA on Human Rights, Democratization and Good Governance', Quebec: CIDA, December. [available at http://acdicida.gc.ca/INET/IMAGES.NSF/vLUImages/HRDG2/\$file/HR DG-Policy-nophoto-e.pdf accessed on 15 November 2011].

Canadian International Development Agency (CIDA) (1999). 'CIDA's Policy on Gender Equality', Quebec: CIDA [available at http://www.sice.oas.org/Genderandtrade/CIDA_GENDERE_Policies.pdf accessed on 9 November 2011]. 
Case, Karl E., Quigley, John M. and Shiller, Robert J. (2005). 'Comparing Wealth Effects: The Stock Market versus the Housing Market', Advances in Macroeconomics, 5:1, Article 1.

Centers for Disease Control and Prevention (CDC) (2000). 'Measuring Healthy Days. Population Assessment of Health-Related Quality of Life', Atlanta: CDC, November.

Chavis, David M. (1983). 'Sense of Community in the Urban Environment: Benefits for Human and Neighborhood Development', doctoral dissertation, Nashville, TN.: Vanderbilt University.

Choden, D., and Namgay, K. (1996). 'Report on the Findings and Recommendations of the Wild Boar Survey: Project for Assessment of Crop Damage by the Wild Boar'. Thimphu: National Plant Protection Centre-Ministry of Agriculture-Royal Government of Bhutan.

Chophel, Sangay. (2010). 'Cultural Diversity and Resilience', Thimphu: The Centre for Bhutan Studies [available at http://ns6.asphostserver.com/surveyReports/culture/culture.pdf accessed on 9 November 2011].

Chouguley, Ulrike, Naylor, Richard and Rosemberg-Montes, Cristina. (2011). 'Edinburgh Festivals Impact Study. Final Report', London: BOP Consulting, May.

Chowdhury, N. and Skarstedt, C.E. (2005). 'The Principle of Good Governance', Oxford: Centre for International Sustainable Development Law (CISDL), March.

Clark, Andrew E. and Senik, Claudia. (2010). 'Who Compare to Whom? The Anatomy of Income Comparisons in Europe', The Economic Journal, 120 (May), 573-594.

Commission of the European Communities. (1992). 'Eurobarometer 38', Brussels: Commission of the European Communities [available at http://ec.europa.eu/public_opinion/archives/eb/eb38/eb38_en.p df accessed on 15 November 2011].

Constitution of Bhutan. (2008). The Constitution of the Kingdom of Bhutan, Thimphu: Constitution of Bhutan [available at http://www.constitution.bt/TsaThrim\%20Eng\%20\%28A5\%29.p df accessed on 15 November 2011].

Cosmides, Leda and Tooby, John. (2000). 'The Cognitive Neuroscience of Social Reasoning'. In Gazzaniga, M. S. (Ed.), The New Cognitive 
Neurosciences, Second Edition. Cambridge, MA: MIT Press, 12591270.

Cox, D., Frere, M., West, S. and Wiseman, J. (2010). 'The Development and Use of Community Wellbeing Indicators: Learning from Community Indicators Victoria', Australian Journal of Social Issues, 45:1, 71-88.

Cummins, R.A. (2000). 'Objective and subjective quality of life: An interactive model', Social Indicators Research, 52, 55-72.

Cummins, R.A. (2003). 'Normative Life Satisfaction: Measurement Issues and a Homeostatic Model'. Social Indicators Research, 64, 225-256.

Cummins, R. A., et al. (2008). 'The Wellbeing of Australians - Who Makes the Decisions, Health/Wealth Control, Financial Advice, and Handedness. Part A: The Report', Victoria: Australian Unity Wellbeing Index (Report 22.0).

Davidson, Richard J. (2004). 'Well-being and Affective Style: Neural Substrates and Biobehavioural Correlates', Philosophical Transactions of the Royal Society B: Biological Sciences, 359, 1395-1411.

Davidson, Richard J., Kabat-Zinn, Jon, Schumacher, Jessica, Rosenkrantz, Melissa, Muller, Daniel, Santorelli, Saki F., Urbanowski, Ferris, Harrington, Anne, Bonus, Katherine and Sheridan, John F. (2003). 'Alterations in brain and immune function produced by mindfulness meditation', Psychosomatic Medicine, 65, 564-570.

Davidson, William B. and Cotter, Patrick R. (1986). 'Measurement of Sense of Community within the Sphere of City', Journal of Applied Social Psychology, 16(7), 608-619.

de Bruin, A.F., de Witte, L.P., Stevens, F. Diederiks, J.P. (1992). 'Sickness Impact Profile: the state of the art of a generic functional status measure.' Soc. Sci. Med. 35, 1003-1014.

De Jesus, Maria, Puleo, Elaine, Shelton Rachel C. and Emmons Karen (2010). 'Associations Between Perceived Social Environment and Neighborhood Safety: Health Implications', Health and Place, 16(5), 1007-1013.

Deaton, Angus. (2007). 'Income, Aging, Health and Wellbeing Around The World: Evidence from the Gallup World Poll', Cambridge, Mass: National Bureau of Economic Research, August (NBER Working Paper Series, No. 13317).

Deaton, Angus. (2010). 'Price Indexes, Inequality, and the Measurement of World Poverty', American Economic Review, 100(1), 5-34. 
Delaney, Liam and Keaney, Emily. (2006). 'Cultural Participation, Social Capital and Civil Renewal in the United Kingdom: Statistical Evidence from National and International Survey Data', London: Institute for Public Policy Research (IPPR), March [available at http://www.ippr.org/uploadedFiles/research/projects/Democrac y $/$ cultural $\% 20$ participation $\% 20$ social $\% 20$ capital $\% 20$ etc.pdf accessed on 24 September 2011].

Department for Environment, Food and Rural Affairs (DEFRA). (2011). 'Life Satisfaction and Other Measures of Wellbeing in England, 2007 - 2011. From the Survey of Public Attitudes and Behaviour Towards the Environment', London: DEFRA (April) [available at http://www.defra.gov.uk/statistics/files/Statistical-Release-13April-2011-wellbeing.pdf accessed on 10 November 2011].

Dessallien, Renata. (2005). Democracy, Good Governance and Happiness. Some Views from the Kingdom of Bhutan. Thimphu: The Centre for Bhutan Studies (Monograph 19).

DeVellis, Robert F. (1991). Scale Development: Theory and Applications. Newbury Park CA: Sage Publications.

Dex, Shirley, Clark, Andrew, and Taylor, Mark. (1995). 'Household Labour Supply', Colchester: University of Essex (Department of Employment Research Series, 43).

Diener, Ed. (1984). ‘Subjective Well-being', Psychological Bulletin, 95(3), 542 575.

Diener, Ed. (2000). 'Subjective Wellbeing: The Science of Happiness and a Proposal for a National Index', American Psychologist, 55(1), 34-43.

Diener, Ed. (2006). 'Understanding Scores on the Satisfaction with Life Scale'. In Diener, Ed, Emmons, Robert A., Larsen, Randy J. and Griffin, Sharon (Eds.), Satisfaction With Life Scale Public Domain, 13 February [available at http://s.psych.uiuc.edu/ ediener/Documents/Understanding $\% 2$ 0SWLS\%20Scores.pdf accessed on 15 September 2011].

Diener, Ed and Diener, Carol. (1996). 'Most People are Happy'. Psychological Science, 7(3), 181-185.

Diener, Ed and Emmons, Robert A. (1984). 'The Independence of Positive and Negative Affect', Journal of Personality and Social Psychology, 47, 1105-1117.

Diener, Ed and Iran-Nejad, A. (1986). 'The Relationship in Experience between Various Types of Affect', Journal of Personality and Social Psychology, 50, 1031-1038. 
Diener, Ed and Pavot, William. (1993). 'Review of the Satisfaction with Life Scale', Psychological Assessment, 5, 164-172.

Diener, Ed. and Seligma, Martin. (2006). 'Measure for Measure: The Case for a National Wellbeing Index', Science and Spirit, March-April, 3637.

Diener, Ed, Emmons, Robert A., Larsem, Randy J. and Griffin Sharon. (1985). 'The Satisfaction with Life Scale', Journal of Personality Assessment, 49(1), 71-75.

Diener, Ed, Helliwell, John F., Kahneman, Daniel (2010). International Differences in Wellbeing, New York: Oxford University Press.

Diener, Ed, Suh, Eunkook and Oishi, Shigehiro (1997). 'Recent Findings on Subjective Well-being', Indian Journal of Clinical Psychology, 24, 2541.

Dipietro, William R. and Anoruo, Emmanuel. (2006). 'GDP per capita and its Challengers as Measures of Happiness', International Journal of Social Economics, 33(10), 698-709.

Diprose, R. (2007). Physical Safety and Security: A Proposal for Internationally Comparable Indicators of Violence. Oxford Development Studies, 35(4), 431-458.

Dixon, Huw David (Ed.) (2000). Controversies in Macroeconomics Growth, Trade and Policy. Oxford: Blackwell.

Dolan, Paul, Peasgood, Tessa, White, Mathew (2008). 'Do We Really Know What Makes us Happy? A Review of the Economic Literature on the Factors Associated with Subjective Wellbeing', Journal of Economic Psychology, 29, 94-122.

Doolittle, R. J. and MacDonald D. (1978). 'Communication and a Sense of Community in a Metropolitan Neighborhood: A Factor Analytic Examination', Communication Quarterly, 26, 2-7.

Doran, S. M., Van Dongen, Hans P. and Dinges, David F. (2001). 'Sustained Attention Performance During Sleep Deprivation: Evidence of State Instability', Archives of Italian Biology: Neuroscience, 139:3, 253-267.

Drèze, Jean, \& Sen, Amartya K. (2002). India: Development and Participation. New Delhi: Oxford University Press.

Duckett, S. and Swerissen, H. (1996). 'Specific Purpose Programs in Human Services and Health: Moving from an Input to an Output and Outcome Focus', Australian Journal of Public Administration, 55, $7-17$. 
Easterlin, Richard A. (2003). 'Explaining Happiness', Proceedings of the National Academy of Sciences, 100(19), 11176-11183.

Economic Commission for Latin America and the Caribbean (ECLAC) (2000). 'Role of Environmental Awareness in Achieving Sustainable Development', Santiago: ECLAC, 23 November [available at http://www.eclac.org/publicaciones/xml/4/8824/lcr1961i.pdf accessed on 10 October 2011].

Egloff, Boris. (1998). 'The Independence of Positive and Negative Affect Depends on the Affect Measure', Personality and Individual Differences, 25(6), 1101-1109.

Ekman, Paul, (1992), 'Are there basic emotions?', Psychological Review, 99(3), $550-3$.

Ekman, Paul, Davidson, Richard J., Ricard, Matthieu and Wallace, B. Alan. (2005). 'Buddhist and Psychological Perspectives on Emotions and Well-Being', American Psychological Society, 14:2, 59-63.

Elvik, R. (1995). 'The Validity of Using Health State Indexes in Measuring the Consequences of Traffic Injury for Public Health', Social Science \& Medicine, 40(10), 1385-98.

Environics (1995). 'The Environmental Monitor: Global Public Opinion on the Environment', brochure.

European Coordination Office (1992). 'Europeans and the Environment. Survey Conducted in the Context of the Eurobarometer 37.0', Brussels: European Coordination Office, August.

Eurostat (2009).Harmonised European Time use Surveys. 2008 Guidelines. Luxembourg: European Communities, (Eurostat methodologies and working papers).

Evans, A. Steven. (2006). 'Preserving the Consciousness of a Nation: Promoting "Gross National Happiness" in Bhutan Through Her Rich Oral Traditions', Journal of Bhutan Studies, 15, Winter.

Everitt, B. (1977). The Analysis of Contingency Tables. London: Chapman and Hall.

Fafchamps, Marcel, \& Shilpi, Forhad Jahan. (2008). Subjective Wellbeing, Isolation and Relative Consumption. Journal of Development Economics, 86, 43-60.

Fagan, Jeffrey and Davies, Garth. (2007). 'The Political Economy of the Crime Decline in New York City', Presented at the Annual 
Meeting of the American Association for Advancement of Science, San Francisco, February.

Feeney, J. A. (1995). 'Adult Attachment and Emotional Control', Personal Relationships, 2, 143-159.

Fiedler, Klaus and Bless, Herbert. (2001). 'The Formation of Beliefs in the Interface of Affective and Cognitive Processes'. In Frijda, Nico, Manstead, Antony and Bem, Sacha (Eds.), The influence of Emotions on Beliefs, New York: Cambridge University Press.

Field, John. (2003). Social Capital. New York: Routledge.

Filmer, Deon and Pritchett, Lant. (2001). 'Estimating Wealth Effects Without Expenditure Data-Or Tears: An Application To Educational Enrollments In States of India', Demography, 38(1), 115-132.

Fischer, Justina AV. (2009). 'Subjective Wellbeing as Welfare Measure: Concepts and Methodology', Paris: OECD, (July) [available at http://mpra.ub.unimuenchen.de/16619/1/MPRA_paper_16619.pdf accessed on 9 September 2011].

Fleming, Robin and Spellerberg, Anne. (1999). Using Time Use Data. A History of Time Use Surveys and Uses of Time Use Data. Wellington: Statistics New Zealand TeTariTatau.

Francis, Paul. (2002). 'Social Capital at the World Bank Strategic and Operational Implications of the Concept', Washington, D. C.: The World Bank, March.

Fredrickson, Barbara L. (2000). 'Cultivating positive emotions to optimize health and well-being', Prevention and Treatment, 3(1).

Frey, Bruno S. and Stutzer, Alois. (2007). 'Should National Happiness Be Maximized?', University of Zurich, March (Working Paper Series, No. 306).

Friedman, Danny. (2010). 'Social Impact of Poor Housing', London: ECOTEC, March.

Fujiwara, Takeo and Kawachi, Ichiro. (2008). 'Social Capital and Health. A Study of Adult Twins in the U.S.', American Journal of Preventive Medicine, 35(2), 139-144.

Galay, Karma (2009). Time Use and Happiness. Thimphu: The Centre for Bhutanese Studies. 
Gallup (2011). 'Environment', Washington, D. C.: Gallup [available at http://www.gallup.com/poll/1615/environment.aspx accessed on 13 November 2011].

Goldberg, D. P and Blackwell, B. (1970). 'Psychiatric Illness in General Practice', British Medical Journal, 2, 438-443.

Gordon, D. (2006). 'The Concept and Measurement of Poverty'. In Pantazis, C., Gordon, D. and R. Levitas (Eds.), Poverty and Social Exclusion in Britain: The Millennium Survey, Bristol: The Policy Press, 29-69.

Graham, Carol. (2005). 'The Economics of Happiness. Insights on Globalization from a Novel Approach', World Economics, 6(3), 4165.

Graham, Carol. (2010). Happiness Around the World: The Paradox of Happy Peasants and Miserable Millionaires. New York Oxford University Press.

Granger, C. V., Cotter, A. C., Hamilton, B. B. and Fiedler, R. C. (1993). 'Functional Assessment Scales: A Study of Persons after Stroke', Archives of Physical Medicine and Rehabilitation, 74(2), 133-138.

Green, Donald Philip, Goldman, Susan L. and Salovey, Peter (1993). 'Measurement Error Masks Bipolarity in Affect Ratings', Journal of Personality and Social Psychology, 64, 1029-1041.

Haque, Umair. (2011). Betterness: Economics for Humans. Boston: Harvard Business Review Press. [available at: http://hbr.org/product/betterness-economics-forhumans/an/11135-PDF-ENG?Ntt=betterness accessed 2 May 2012].

Harvey, Carol, Pantelis, Christos, Taylor, Jason, McCabe, Patrick, Lefevre, Karen, Campbell, Patrick and Hirsch, Steven R. (1996). 'The Camden Schizophrenia Surveys: II. High Prevalence of Schizophrenia in an Inner London Borough and its Relationship of Sociodemographic Factors', British Journal of Psychiatry, 168, 418426.

Heberlein, Thomas A. (2005). 'Environmental Attitudes', Abhandlungen, 81(2), 241-270 [available at http://www.drs.wisc.edu/documents/articles/heberlein/Environ mentalAttitudes.pdf accessed on 24 September 2011].

Hedley, T. P. (1998). 'Measuring Public Sector Effectiveness Using Private Sector Methods', Public Productivity and Management Review, 21, 251258. 
Helliwell, J. F., and Wang, S. (2011). 'Trust and Wellbeing', International Journal of Wellbeing, 1(1), 42-78.

Helliwell, John, Layard, Richard, Sachs, Jeffrey (Eds.) (2012). World Happiness Report. New York: Earth Institute, Columbia University. [available at: http://www.earth.columbia.edu/sitefiles/file/Sachs\%20Writing/ 2012/World\%20Happiness\%20Report.pdf accessed 2 May 2012].

Hennessy, Catherine Hagan, Moriarty, David G., Zack, Matthew M., Scherr, Paul A. and Brackbill, Robert. (1994). 'Measuring Healthrelated Quality of Life for Public Health Surveillance', Public Health Reports, 109, 665-672.

Hillyard, P., Kelly, G., McLaughlin, E., Patsios, D. and Tomlinson, M. (2003), Bare Necessities: Poverty and Social Exclusion in Northern Ireland - Key Findings, Belfast: Democratic Dialogue.

Hötte, Michiel and Bereznuk Sergei (2001). 'Compensation for Livestock Kills by Tigers and Leopards in Russia', Carnivore Damage Prevention News, 3, 6-7.

Huppert, Felicia A. (2005). 'Positive Emotions and Cognition: Developmental, Neuroscience and Health Perspectives'. Hearts and minds: Affective Influences on Social Cognition and Behavior. New York: Psychology Press.

Ibrahim, S., \& Alkire, S. (2007). 'Agency and Empowerment: A Proposal for Internationally Comparable Indicators'. Oxford Development Studies, 35(4), 379-403.

Iizuka, Michiko. (2000). 'Role of Environmental Awareness in Achieving Sustainable Development'. Santiago: Economic Commission for Latin America and the Caribbean [available at http://www.eclac.org/publicaciones/xml/4/8824/lcr1961i.pdf accessed on 14 November 2011].

International Labour Organisation (ILO). (1919). Convention Limiting the Hours of Work in Industrial Undertakings to Eight in the Day and Fortyeight in the Week, Washington: ILO.

Inter-university Consortium for Political and Social Research (ICPSR). (1996). 'International Social Survey Program: Environment, 1993'. Ann Arbor: ICPSR.

Ironmonger, D. ( 1999). 'An Overview of Time Use Surveys'. Paper presented at UNESCAP Time Use Seminar Ahmedabad, India, 710 December 1999. Available at <http: //www.economics.unimelb.edu.au/ Household / Papers/1999/ AhmedabadPaper2.pdf $>$ [Accessed on 19.1.2012] 
Itay, Anat (2009). 'Israel's Progress Index: A Multi-Layered Model for Measuring Progress and Quality Of Life' presented in The 3rd OECD World Forum on 'Statistics, Knowledge and Policy' Charting Progress, Building Visions, Improving Life, Busan 27-30 October [Available at: http://www.oecd.org/dataoecd/53/20/44096120.pdf, accessed on 6 September 2011].

Jabeen, Nasira (2007). 'Good or Good Enough Governance in South Asia: Constraints and Possibilities', Address at Utrecht University, April 2.

Johns, Helen and Ormerod, Paul. (2007). Happiness, Economics and Public Policy. London: The Institute of Economic Affairs.

Johns, Helen and Ormerod, Paul. (2008). 'The Unhappy Thing about Happiness Economics', Real-world Economics Review, 46, 139-146 [Available at http://www.paecon.net/PAEReview/issue46/JohnsOrmerod46.p df accessed on 9 September 2011].

Johns, Helen and Ormerod, Paul. (2009). 'Reply to Dan Turton'. Realworld Economics Review, 49, 91-93. Available at [http://www.paecon.net/PAEReview/issue49/Johns49.pdf accessed on 9 September 2011].

Jylhä, Marttila. (2009). 'What is Self-rated Health and Why Does it Predict Mortality? Towards a Unified Conceptual Model', Social Science and Medicine; 69(3), 307-16.

Kahneman, D. and Krueger, A. B. (2006). 'Developments in the Measurement of Subjective Wellbeing', Journal of Economic Perspectives, 20(1), 3-24.

Kahneman, Daniel and Deaton, Angus. (2010). 'High Income Improves Evaluation of Life but Not Emotional Wellbeing', Proceedings of the National Academy of Sciences of the United States of America, September [available at www.pnas.org/cgi/doi/10.1073/pnas.1011492107 accessed on 9 September 2011].

Kahneman, Daniel, Krueger, Alan B., Schkade, David, Schwarz, Norbert and Stone, Arthur A. (2006). 'Would You Be Happier If You Were Richer? A Focusing Illusion', Brussels: The Centre for European Policy Studies, May (CEPS Working Paper No. 125) [Available at: http://www.princeton.edu/ceps/workingpapers/125krueger.pdf accessed on 9 November 2011]. 
Kahneman, Daniel, Krueger, Alan B., Schkade, David, Schwarz, Norbert and Stone, Arthur. (2004). 'The Day Reconstruction Method (DRM): Instrument Documentation' [Available at www.krueger.princeton.edu/drm_documentation_july_2004.pdf accessed on 15 September 2011].

Kahneman, David, Fredrickson, Barbara L., Schreiber, Charles. A. and Redelmeier, Donald A. (1993). 'When More Pain is Preferred to Less: Adding a Better End', Psychological Science, 4, 401-405.

Kalmijn, W. M.; Arends, L. R. and Veenhoven, R. (2011). 'Happiness Scale Interval Study. Methodological Considerations', Social Indicators Research, 102, 497-515.

Kaplan, R. M. and Bush, J. W. (1982). 'Health-related Quality of Life Measurement for Evaluation Research and Policy Analysis', Health Psychology, 1, 61-80.

Kaufmann, Daniel, Kraay, Aart and Mastruzzi, Massimo. (2005). 'Governance matters IV: governance indicators for 1996-2004', Washington, D. C.: The World Bank (Policy Research Working Paper Series 3630).

Kendall, M. (1948). Rank Correlation Methods. London: Charles Griffin \& Company.

Kendall, M. (1975). Multivariate Analysis. London: Charles Griffin \& Company.

Kenny, Anthony, and Kenny, Charles. (2006). Life, Liberty and the Pursuit of Utility: Happiness in Philosophical and Economic Thought. Exeter: Imprint Academic.

Kinga, Sonam. (2001). 'The Attributes and Values of Folk and Popular Songs', Journal of Bhutan Studies, 3, 132-170.

Kleinman, Arthur (1995). Writing at the Margin: Discourse Between Anthropology and Medicine. Berkeley: University of California Press.

Kleitman, Nathaniel. (1963). Sleep and Wakefulness. Chicago: Chicago University Press, $2^{\text {nd }}$ edition.

Larsen, Jeff T., McGraw, A. Peter and Cacioppo, John T. (2001). 'Can People Happy and Sad at the Same Time?' Journal of Personality and Social Psychology, 81, 684-696.

Layard, Richard. (2005). Happiness: Lessons from a New Science. London: Penguin. 
Leahy, Robert L. (1997). 'Introduction: Fundamentals of Cognitive Therapy'. In Leahy, Robert L. (Ed.), From Practicing Cognitive Therapy: A Guide to Interventions, Northvale: Jason Aronson, 1-11.

Lee, Sangheon, McCann, Deirdre and Messenger, Jon C. (2007).Working Time Around the World. Trends in working Hours, Laws and Policies in a Global Comparative Perspective. New York: Routledge - International Labour Organization.

Legatum Institute (2010). 'Methodology. The Legatum Prosperity Index', Dubai: The Legatum Institute.

Lepper, Jonathan and McAndrew, Siobhan (2008). 'Developments in the Economics of Wellbeing', London: HM Treasury, November (Treasury Economic Working Paper, No.4).

Levine, Edward L. and Xu, Xian. (2005). 'Development and Validation of the State-Trait Emotion Measure (STEM)'. Paper presented at the 20th Annual Conference of the Society for Industrial and Organizational Psychology, Los Angeles, April.

Lupton, Ruth and Power, Anne. (2005). 'Disadvantaged by Where you live? New Labour and Neighbourhood Renewal'. In Hills, John and Stewart, Kitty (Eds), A More Equal Society? New Labour, Poverty, Inequality and Exclusion. Bristol: The Policy Press.

Mahoney F. I. and Barthel D. (1965). 'Functional evaluation: the Barthel Index', Maryland State Medical Journal, 14, 56-61.

McBride, Michael (2010). 'Money, Happiness, and Aspirations: An Experimental Study', Journal of Economic Behaviorand Organization, 74, 262-276.

McDowell I. and Newell, C. (1996). Measuring Health: A Guide to Rating Scales and Questionnaires, New York: Oxford University Press, Second edition.

McGillivray, Mark and Clarke, Matthew (Eds.) (2006). Understanding Human Well-being. New York: United Nations University Press.

McHorney, Colleen A. (1999). 'Health Status Assessment Methods for Adults Past Accomplishments and Future Challenges', Annual Review of Public Health, 20, 309-335.

McHorney, Colleen A., Ware, J. E. and Raczek, A. E. (1993). 'The MOS 36-Item Short-Form Health Survey (SF-36): II. Psychometric and Clinical Tests of Validity in Measuring Physical and Mental Health Constructs', Medical Care, 31, 247-263. 
McMillan, David. (1976). 'Sense of Community: An Attempt at Definition'. Unpublished manuscript. Nashville, TN.: George Peabody College for Teachers, Vanderbilt University.

Michalos, Alex, Sharpe, Andrew, Arsenault, Jean-François, Muhajarine, Nazeem, Labonté, Ronald, et al. (2010). 'An Approach to the Canadian Index of Wellbeing. Methodology Report', Waterloo: The Canadian Index of Wellbeing Network - University of Waterloo.

Monroe, S., Jabine, T., Willis, G., Martin, E. and Tucker, Clyde. (Eds.) (1999). 'A New Agenda for Interdisciplinary Survey Research Methods Centers for Disease Control and Prevention', Hyattsville: Centers for Disease Control and Prevention - U. S. National Centre for Health Statistics, January 1999.

Montgomery, Mark R., Gragnolati, Michele, Burke, Kathleen A., and Paredes, Edmundo. (2000). 'Measuring Living Standards With Proxy Variables', Demography, 37(2), 155-74.

Morris, Saul S., Carletto, Calogero, Hoddinott, John and Christiaensen, Luc J. M. (2000). 'Validity of Rapid Estimates of Household Wealth and Income for Health Surveys in Rural Africa', Journal of Epidemiology and Community Health, 54, 381-387.

National Institute of Statistics and Economic Studies (Institut National de la Statistique et des Études Économiques: INSEE). (2011). Two years after the Stiglitz-Sen-Fitoussi report: What well-being and sustainability measures? Paris: INSEE. [Available at: http://www.insee.fr/en/publications-etservices/default.asp?page $=$ dossiers_web/stiglitz/stiglitz_intervent ion.htm accessed 2 May 2012].

Nesse, Randolph M. (2004). 'Natural Selection and the Elusiveness of Happiness', Philosophical Transactions of the Royal Society B: Biological Sciences, 359, 1333-1347.

Nunnally, Jum C. (1978). Psychometric Theory. New York: McGraw-Hill, 2nd edition.

OECD. (1995). 'Toward a Framework for Re-evaluating the Policy Implications of Unpaid Work', Paris: Working Party on the Role of Women in the Economy 20th Meeting, October.

OECD (2009). 'Society at a Glance. OECD Social Indicators. Special Focus: Measuring Leisure in OECD Countries'. Paris: OECD.

OECD (2010). Eurostat: Feasibility study for Subjective Wellbeing indicators. Task 4: Critical review. [Available at http://epp.eurostat.ec.europa.eu/portal/page/portal/gdp_and_be 
yond/documents/

Feasibility_study_Wellbeing_Indicators.pdf accessed on 9

September 2011].

OECD (2011). 'Key Findings on Chapter 1: Unpaid Work' in OECD, Society at a Glance 2011 - OECD Social Indicators, 12 April [Available at www.oecd.org/els/social/indicators/SAG accessed on 22 September 2011].

OECD. (2008). Handbook on Constructing Composite Indicators: Methodology and User Guide. Paris: OECD

OECD. (2011a). Your Better Life Index. [Available at: http://oecdbetterlifeindex.org/ accessed 2 May 2012].

OECD. (2011b). How's life? Measuring well-being. Paris: OECD. [available at http://www.oecd.org/document/10/0,3746,en_2649_201185_487 91306_1_1_1_1,00.html accessed 2 May 2012].

Office for National Statistics (2011). 'Measuring Wellbeing. Measuring What Matters? National Statistician's Reflections on the National Debate on Measuring National Wellbeing', London: ONS (July).

Oswald, Andrew (2010). 'Emotional Prosperity, not GDP', The Economist, April.

Paloutzian, Raymond F. and Ellison, Craig W. (1982). Manual for the Spiritual Well-being Scale. Nyack, NY: Life Advance, Inc.

Parke, Ross D. and O'Neil, Robin L. (1999). 'Neighborhoods of southern California children and families', Future of Children, 9(2), 58-63.

Pavot, William and Diener, Ed. (1993). 'Review of the Satisfaction with Life Scale', Psychological Assessment, 5(2), 164-172.

Penjore, Dorji, and Rapten, Phuntsho. (2008). 'Political Pursuit of Gross National Happiness', Bangkok: International Conference on 'Buddhism in the Age of Consumerism', Mahidol University (1-3 December).

Pentland, Wendy E. and McColl, Mary Ann (1999). 'Application of Time Use Research to the Study of Life with a Disability'. In Pentland, Wendy E., Harvey, Andrew S., Lawton, M. Powell, and McCoil, Mary Ann (Eds.), Time Use Research in the Social Sciences, New York: Kiuwer Academic / Plenum Publishers, 169-188.

Prakke, Diederik (2005). 'The Buddhist Truth of Happiness, Spirituality and Development. The Case of Governance in Bhutan', Journal of Bhutanese Studies, 12, 119-165. 
Pudney, Stephen. (2011). 'Perception and Retrospection: The Dynamic Consistency of Responses to Survey questions on

Wellbeing', Journal of Public Economics, 95(3-4), 300-310.

Putnam, Robert D. (1993). Making Democracy Work. Civic Traditions in Modern Italy. Princeton: Princeton University Press.

Putnam, Robert D. (2000). Bowling Alone: the Collapse and Revival of American Community, New York: Simon and Schuster.

Qi, Yaqiang, Hu, Peifeng and Mason, William M. (2009). 'A Comparative Analysis of Self-Rated General Health in Three Developing Countries'. Paper presented at the Annual Meeting of the Population Association of America, Detroit 29 April-2 May.

Rahman, Omar, Menken, Jane and Kuhn, Randall. (2004). 'The Impact of Family Members on Self-Reported Health of Elderly Men and Women in Rural Bangladesh', Ageing and Society, 24, 903-920.

Rapten, Phuntsho (2010). Good Governance and Gross National Happiness. Thimphu: The Centre for Bhutan Studies [available at http://www.grossnationalhappiness.com/surveyReports/goodGo vernance/Good_Governance_final.pdf accessed on 14 September 2011].

Reed, Pamela. (1987). 'Spirituality and Well-Being in Terminally Ill Hospitalized Adults', Research in Nursing and Health, 10, 335-344.

Renn, Daniela, Pfaffenberger, Nicole, Platter, Marion, Mitmansgruber, Horst, et al. (2009). 'International Wellbeing Index: The Austrian Version', Social Indicators Research, 90, 243-256.

Riger, S. and Lavrakas, P. (1981). 'Community Ties: Patterns of Attachment and Social Interaction in Urban Neighbourhoods', American Journal of Community Psychology, 9, 55-66.

Rinzin, Chhewang, Vermeulen, Walter J. V. and Glasbergen, Pieter. (2007). 'Public Perceptions of Bhutan's Approach to Sustainable Development in Practice', Sustainable Development, 15, 52-68.

Robinson, John and Godbey, Geoffrey. (1997). Time For Life. The Surprising Ways Americans Use Their Time. University Park, PA: The Pennsylvania State University Press.

Rojas, Mariano. (2011). 'The "Measurement of Economic Performance and Social Progress" Report and Quality of Life: Moving Forward.' Social Indicators Research. 102(1), 169-180.

Rondinella, Tommaso, Segrez, Elisabetta, Mascherinix, Massimiliano. (2011). 'Wellbeing in Italian Regions. Measures, Civil Society 
consultation and Evidence', Social Indicators Research, 102(1), 4769.

Rosenberg, Erika L. and Ekman, Paul. (1994). 'Coherence between Expressive and Experiential System of Emotions', Cognition and Emotion, 8, 201-229.

Royal Government of Bhutan (1999). Bhutan 2020: A Vision for Peace, Prosperity and Happiness. Thimphu: The Planning Commission [available at http://unpan1.un.org/intradoc/groups/public/documents/apcity /unpan005249.pdf accessed on 15 November 2011].

Royal Government of Bhutan (2000). Bhutan National Human Development Report 2000. Thimphu: The Planning Commission Secretariat [available at http://unpan1.un.org/intradoc/groups/public/documents/apcity /unpan005248.pdf accessed 15 November 2011].

Royal Government of Bhutan (2007). Poverty Analysis Report 2007. Thimphu: National Statistics Bureau [Available at http://www.undp.org.bt/assets/files/publication/PAR_2007.pdf accessed on 15 November 2011].

Royal_Government_of_Bhutan (2008a). Bhutan Tenth Round Table Meeting Report In G. N. H. Commission (Ed.) (February 17-18): Thimphu.

Royal_Government_of_Bhutan (2008b). Tenth Five Year Plan [2008-2013] (Vol. 1). Thimphu: Gross National Happiness Commission.

Royal Government of Bhutan (2009). Tenth Five-Year Plan. 2008-2013. Thimphu: Gross National Happiness Commission [Available at http://www.unpei.org/PDF/BhutanTenthFiveYearPlan_GrossNationalHappinessCommission.pdf accessed on 15 November 2011].

Royal Government of Bhutan. (2011b). Eleventh Roundtable Meeting Turning Vision Into Reality: The Development Challenges Facing Bhutan. Thimphu: Gross National Happiness Commission (2011a). Bhutan National Human Development Report 2011: Sustaining Progress: Rising to the Climate Challenge. Thimphu: Gross National Happiness Commission.

Russell, James A. and Carroll, James M. (1999). 'On the Bipolarity of Positive and Negative Affect', Psychological Bulletin, 125, 3-30. 
Ryan, Richard M., and Deci, Edward L. (2001). 'On Happiness and Human Potentials: A Review of Research on Hedonic and Eudaimonic Well-being', Annual Review of Psychology, 52, 141-166.

Saamah, Abdallah, Sam Thompson, Juliet Michaelson, Nic Marks and Nicola Steuer (2009). Happy Planet Index 2.0. London: The New Economic Foundation.

Sachs, Jeffrey. (2011). The Price of Civilization: Reawakening American Virtue and Prosperity. New York: Random House.

Samman, E. (2007). Psychological and Subjective Well-being: A Proposal for Internationally Comparable Indicators. Oxford Development Studies, 35(4), 459-486.

Schimmel, Jiirg. (2009). 'Development as Happiness: The Subjective Perception of Happiness and UNDP's Analysis of Poverty, Wealth and Development', Journal of Happiness Studies, 10, 93-111.

Schwartz, Shalom H. (1992). 'Universals in the Content and Structure of Values: Theoretical Advances and Empirical Tests in 20 Countries', Advances in Experimental Social Psychology, 25, 1-65.

Schwartz, Shalom H. (2002). 'Value Priorities and Behaviour: Applying a Theory of Integrated Value Systems', Psicodebate: Psicologia, Cultura y Sociedad, 2, 119-144.

Schwarz, Norbert (2007). 'Retrospective and Concurrent Self-Reports: The Rationale for Real-Time Data Capture'. In Stone, A., Shiffman, S. S., Atienza, A., and Nebeling, L. (Eds.), The science of Real-Time Data Capture: Self-Reports in Health Research. New York: Oxford University Press, pp. 11-26.

Schwarz, Norbert (2011). 'Why Researchers Should Think 'Real-Time': A Cognitive Rationale'. Preliminary draft for M. R. Mehl and T. S. Conner (Eds.), Handbook of Research Methods for Studying Daily Life, New York: Guilford.

Sen Gupta, B. (2009). Bhutan: Towards a Grass-Root Participatory Polity. New Delhi: The Center for Studies in Global Change, 1999.

Sen, Amartya K. (1993). Capability and Well-being. In M. Nussbaum \& A. Sen (Eds.), The Quality of Life. Oxford: Clarendon Press.

Sen, Amartya K. (1999). Development as Freedom (1st. ed.). New York: Knopf.

Sen, Amartya K. (2000). 'The living Standard', In T. Cowen (Ed.), Economic Welfare (Vol. 3, pp. 110-126). Cheltenham, UK and Northampton, MA: Elgar. 
Sen, Amartya K. (2002). 'Health: Perception versus Observation. Selfreported Morbidity has Severe Limitations and Can be Extremely Misleading', British Medical Journal, 324, 860-861.

Sen, Amartya K. (2003). 'Human Security Now', SGI Quarterly, 2-5

Sen, Amartya K. (2004). Elements of a Theory of Human Rights. Philosophy and Public Affairs, 32(4), 315-356.

Sen, Amartya K. (2006a,). 'Democracy Isn't “Western”', The W all Street Journal. March 24.

Sen, Amartya K. (2006b). Identity and Violence: The Illusion of Destiny (1st ed.). London: Penguin Books.

Sen, Amartya. (2009). The Idea of Justice. London: Penguin.

Siegel, S. and Castellan Jr, N. J. (1988.) Nonparametric Statistics for the Behavioral Sciences. 2nd edition. London: McGraw-Hill.

Singleton, Jerome F. and Harvey, Andrew (1995). 'Stage of Lifecycle and Time Spent in Activities', Journal of Occupational Science, 2(1), 3-12.

Smith, Melinda, Robinson, Lawrence, and Segal, Robert. (2011). 'How Much Sleep Do You Need?, Helpguide.org, October [Available at http://helpguide.org/life/sleeping.htm accessed on 10 November 2011].

Sorber, A. (1993). 'Performance Measurement in the Central Government Departments of the Netherlands', Public Productivity and Management Review,17(1), 59-68.

Sorokin, Pitirim and Berger, Clarence (1939). Time-budgets of Human Behavior. Cambridge, Mass.: Harvard University Press.

Sousa, L., Lyubomirsky, S. (2001). 'Life satisfaction'. In J. Worell (Ed.), Encyclopedia of Women and Gender: Sex Similarities and Differences and the Impact of Society on Gender. Volume 2, San Diego, CA: Academic Press, 667-676.

Staines, Graham L. and Pleck, Joseph H. (1983). The impact of work schedules on the family. Ann Arbor: University of Michigan-Institute for Social Research.

Stark, Oded. (1995). Altruism and Beyond: An Economic Analysis of Transfers and Exchanges within Families and Groups. Cambridge: Cambridge University Press.

Stein, R. E., \& Jessop, D. J. (1990). 'Functional Status II(R): A Measure of Child Health Status', Medical Care, 28, 1041. 
Steinmann, Ralph M. (2010). 'Spirituality - The Fourth Dimension of Health. An Evidence-Based Definition'. Presentation in the 20th IUHPE World Conference on Health Promoting Theory: Missing Elements and New Approaches, 11-15 July.

Stiglitz, Joseph, Sen, Amartya K, Fitoussi, Jean-Paul (2009a). Report by the Commission on the Measurement of Economic Performance and Social Progress. Paris: Commission on the Measurement of Economic Performance and Social Progress (14 September) [Available at http://www.stiglitz-sen-fitoussi.fr/documents/rapport_anglais.pdf accessed on 14 September 2011].

Stiglitz, Joseph, Sen, Amartya K, Fitoussi, Jean-Paul. (2009b). The Measurement of Economic Performance and Social Progress Revisited. Reflections and Overview. Paris: Commission on the Measurement of Economic Performance and Social Progress (16 September) [Available at http://www.stiglitz-senfitoussi.fr/documents/overview-eng.pdf accessed on 14 September 2011].

Stock, William A. and Okun, Morris A. (1982). 'The Construct Validity of Life Satisfaction among the Elderly', Journal of Gerontology, 37(5), $625-27$.

Stone, Melissa M. and Cutcher-Gershenfeld, Susan (2001). 'Challenges of Measuring Performance in Nonprofit Organizations'. In Flynn, Patrice and Hodgkinson, Virginia A. (Eds.), Measuring the Impact of the Non-profit Sector. New York: Kluwer Academic, 33-54.

Szalai, Sandor. (1972). The Use of Time: Daily Activities of Urban and Suburban Populations in Twelve Countries. The Hague: Mouton.

Tara, Green, Two Meditation Practices. [Available at www.losangsamten.com/sadhana/green_tara_2.pdf accessed on 15 September 2011].

The EuroQol Group (1990). 'EuroQol--A New Facility for the Measurement of Health-related Quality of Life', Health Policy, 16(3), 199-208.

The United Nations Environment Programme and Louis Harris and Associates (1989). Harris 1989 Environmental Survey in Four Continents. Study no. 884002. New York: Louis Harris and Associates [Available at http://hdl.handle.net/1902.29/H-884002 accessed on 15 November 2011].

The World Bank (1992). Governance and Development, Washington, DC: World Bank. 
Thinley, Lyonpo Jigmi Y. (1998). 'Values and Development: Gross National Happiness', Seoul: The Millennium Meeting for Asia and the Pacific, 30 October-1 November.

Thirgood, Simon J. and Redpath, S. M. (2008). 'Hen Harriers and Red Grouse: Science, Politics and Human-Wildlife Conflict', Journal of Applied Ecology, 45, 1550-1554.

Tomkins, Silvan S. (1981). 'The Quest for Primary Motives: Biography and Autobiography of an Idea', Journal of Personality and Social Psycbology, 41(2), 306-329.

Tooby, John and Cosmides, Leda. (2000). 'Toward Mapping the Evolved Functional Organization of Mind and Brain'. In Gazzaniga, Michael S. (Ed.), The New Cognitive Neurosciences, Second Edition. Cambridge, MA: MIT Press, 1167-1178.

Torenvlied, René and Akkerman, Agnes. (2009a). 'Organizational Environment: Effects of Network Ambition on Agency Performance'. Paper prepared for presentation at the 10th Public Management Research Conference (PMRC), Columbus, Ohio, October 1-3.

Torenvlied, René and Akkerman, Agnes. (2009b). 'Subjective and Objective Agency Performance: A Multilevel—Multistage Approach'. Paper prepared for presentation at the 10th Public Management Research Conference (PMRC), Columbus, Ohio, October 1-3.

Turton, Dan. (2009). 'The Real Dirt on Happiness Economics: A Reply to :The Unhappy Thing about Happiness economics"', Real-world Economics Review, 49, March, 83-90[Available at http://www.paecon.net/PAEReview/issue49/Turton49.pdf accessed on 9 September 2011].

U. S. Department of Health and Human Services. (2000). Healthy People 2010, Washington, DC: U.S. Government Printing Office.

UNDP. (2010). Human Development Report 2010: The true Wealth of Nations: Pathways to Human Development. New York.

UNDP Bhutan (2008). 'Bhutan's Progress: Midway to the Millennium Development Goals', Thimphu: UNDP. [Available at www.undp.org.bt/assets/files/publication/MDG_Midway.pdf accessed on 10 October 2011].

United States Bureau of Labor Statistics (BLS) (2010). American Time Use Survey (ATUS) - 2009 Results. [Available at 
http://www.bls.gov/news.release/archives/atus_06222010.pdf accessed on 22 September 2011].

United States Government Accountability Office (2011). 'Key Indicator Systems. Experiences of Other National and Subnational Systems Offer Insights for the United States', GAO- Report to Congressional Addressees. (GAO 11-396) Washington, D. C.: GAO.

Ura, Karma, and Zangmo, Tshoki. (2008). 'An Approach to the Indicators of GNH'. Document presented at the Regional Conference on 'Revitalizing Primary Health Care' in Jakarta. World Health Organization, 6-8 August. [Available at http://www.searo.who.int/LinkFiles/Conference_Panel-B3.pdf accessed on 9 November 2011].

Ura, Karma. (2009). 'Live Interview with Dasho Karma Ura by the Bhutan Broadcasting Service (BBS) Radio Programme', Thimphu: BBS, 14 September.

Ura, Karma. (2009). A Proposal for GNH V alue Education. Thimphu, Bhutan.

Ura, Karma. (2011a). Explanation of the GNH Index. Thimphu: The Centre for Bhutan Studies [Available at http://www.grossnationalhappiness.com/gnhIndex/intruduction GNH.aspx accessed on 9 September 2011].

Ura, Karma. (2011b). The Bhutanese Development Story. Thimphu: The Centre for Bhutan Studies [Available at www.bhutanstudies.org.bt/pubFiles/mono-1en-bt-dev-stry.pdf accessed on 14 September 2011].

Van de Walle, Steven and Bouckaert, Geert. (2003). 'Public service performance and trust in government: the problem of causality', International Journal of Public Administration, 29(8,9), 891-913.

Van Eerden, Mennobart R. (1990). 'The Solution of Goose Damage Problems in the Netherlands, with special Reference to Compensation Schemes', Ibis, 132, 253-261.

Van Hoorn, Andre (2007). 'A Short Introduction to Subjective Well-Being: Its Measurement, Correlates And Policy Uses'. Paper prepared for the international conference 'Is happiness Measurable and What do Those Measures Mean for Policy?' Rome.

Van Praag, Bernard M. S. (2007). 'Perspectives from the Happiness Literature and the Role of New Instruments for Policy Analysis', CESifo Economic Studies, 53(1), 42-68. 
Varni, J. W., Seid, M., Kurtin, P. S. (1999). 'Pediatric Health-related Quality of Life Measurement Technology: A Guide for Health care Decision Makers', Journal of Clinical Outcomes Management, 6, 33-40.

Veenhoven, Ruut. (1991). 'Is Happiness Relative?', Social Indicators Research, $24,1-34$.

Veenhoven, Ruut. (2004). 'Happiness as an Aim in Public Policy. The Greatest Happiness Principle'. In Linley, Alex and Joseph, Stephen (Eds.), Positive Psychology in Practice, Chapter 39.

Veenhoven, Ruut. (2007). 'Quality-of-Life Research'. In Bryant, C.D. and Peck, D.L., 21st Century Sociology, A Reference Handbook. Volume 2, Thousand Oaks: Sage, 54-62.

Wandersmand, A. and Giamartino, G. A. (1980). 'Community and Individual Difference Characteristics as Influences on Initial Participation', American Journal of Community Psychology, 8, 217-228.

Wang, Sonam W., Curtis, Paul D. and Lassoie, James P. (2006). 'Farmer Perceptions of Crop Damage by Wildlife in Jigme Singye Wangchuck National Park', Wildlife Society Bulletin, 34(2), 359-365.

Wangchuk, Dorji (2008). 'Country Report: Bhutan'. Document prepared for the 'Training Course for Safeguarding of Intangible Cultural Heritage', Tokyo: Asia/Pacific Cultural Centre for UNESCO, 1117 December [Available at http://www.accu.or.jp/ich/en/training/country_report_pdf/08_0 $9 /$ country_report_bhutan_03.pdf accessed on 24 September 2011].

Wangdi, Karma (2009). Education Indicators. Thimphu: The Centre for Bhutan Studies.

Wangdi, Karma (2009). Health Indicators. Thimphu: The Centre for Bhutan Studies.

Wangyal, Tenzin (2001). 'Enormous Compassion. An interview with Tenzin Wangyal Rinpoche', Voice of Clear Light, Shipman VA: Ligmincha Institute September 18 [Available at http://www.snowlionpub.com/pages/wangyalteaching1.html accessed on 14 November 2011].

Ware, J. E. (1995). 'The Status of Health Assessment 1994', Annual Review of Public Health, 16, 327-54.

Ware, J.E. and Sherbourne, C.D. (1992). 'The MOS 36-item Short-form Health Survey (SF-36)', Medical Care, 30(6), 473-483. 
Watson, David and Tellegen, Auke. (1999). 'Issues in the Dimensional Structure of Affect: Effects of Descriptors, Measurement Error, and Response Formats: Comment on Russell and Carroll (1999)', Psychological Bulletin, 125, 601-610.

Watson, David, Clark, Lee A., and Tellegen, Auke. (1988). 'Development and Validation of Brief measures of Positive and Negative Affect: The PANAS Scales', Journal of Personality and Social Psychology, 47, 1063-1070.

Watson, David; Wiese, D.; Vaidya, J., and Tellegen, Auke. (1999). 'The Two general Activation Systems of Affect: Structural Findings, Evolutionary Considerations, and Psychobiological evidence', Journal of Personality and Social Psychology, 76, 820-838.

Weitz-Shapiro, Rebecca and Winters, Matthew S. (2008). 'Political Participation and Quality of Life'. Working Paper, 638.Washington, D. C.: Inter-American Development Bank.

White, Michael J. and Hunter, Lori M. (2005). 'Public Perception of Environmental Issues in a Developing Setting'. Providence: Brown University, 17 May [Available at http://www.pstc.brown.edu/ghana/Papers/EnvPerc_White_Hun ter.pdf accessed on 24 September 2011].

Wilkinson, Diana. (1999). 'Poor Housing and Ill Health. A Summary of Research Evidence'. Edinburgh: The Scottish Office - Central Research Unit [available at http://scotland.gov.uk/Resource/Doc/156479/0042008.pdf accessed on 10 October 2011].

Wiseman, John, and Brasher, Kathleen (2008). 'Community Wellbeing in an Unwell World: Trends, Challenges and Possibilities', Journal of Public Health Policy, 29, 353-366.

World Health Organization (2010) 'International Workshop on Housing, Health and Climate Change: Developing Guidance for Health Protection in the Built Environment Mitigation and Adaptation Responses'. Geneva: WHO, 13-15 October.

Yalonetzky, G. (2010). 'A note on the Standard Errors of the Members of the Alkire-Foster Family and its Components'. Mimeo.

Yoshida, Rie and Nakano, Sachiko (2007). 'Changes and Trends in Media Use: From the Results of the 2005 Japanese Time Use Survey', NHK Broadcasting Studies, 5, 117-142 [Available at http://www.nhk.or.jp/bunken/english/reports/pdf/0607_no5_07.pdf accessed on 10 November 2011]. 
Zavaleta, D. (2007). 'The Ability to Go about Without Shame: A Proposal for International Comparable Indicators on Shame and Humiliation', Oxford Development Studies, 35(4), 405-430.

Zeppa, Jaime. (2006). 'Surviving Happiness. Can happiness be a Gross National Product? The Myth and Reality of "Progress" in Bhutan', Ascent Magazine, 32, Winter.

Zurick, David (2006). 'Gross National Happiness and Environmental Status in Bhutan', Geographical Review, 96, 4, 657-681. 


\section{Appendix 1. Sampling and weighting}

\section{Generation of Sample Weights}

As the GNH survey was not self-weighting, the final survey results were determined based on a multistage probability weighting method. Weighting for household surveys involved three operations-calculation of selection probabilities, adjustments for non-response, and calculation of the base or design weights.

\section{Calculation of selection probabilities; probability proportionate to estimated size}

The major component of the weight is the reciprocal of the sampling fraction employed in selecting the number of sample households in that particular sampling stratum (h) and Primary Selection Unit (PSU):

$$
W_{h i}=\frac{1}{f_{h i}}
$$

The term $f_{b i}$, the sampling fraction for the $i^{\text {th }}$ sample PSU in the $b^{\text {th }}$ stratum is the product of probabilities of selection at every stage in each sampling stratum:

$$
f_{h i}=p_{1 h i} \times p_{2 h i}
$$

where $p_{s b i}$ is the probability of selection of the sampling unit at stage $s$ for the $i^{\text {th }}$ sample PSU in the $b^{\text {th }}$ sampling stratum. The following steps for the calculation of the selection probabilities were used:

1. The probability of selection in each PSU within the stratum $=$ (Number of PSUs in each stratum) * (Number of households in each PSU in PHC / Number of households in each dzongkhag in PHC)

2. Probability of selection of each household $=$ Number of households selected in each PSU/Number of households listed

Since the estimated number of households in each enumeration area (PSU/cluster) in the sampling frame used for the first stage selection differed from the updated number of households in the enumeration area from the listing, individual sampling fractions for households in each sample enumeration area (cluster) were calculated. The sampling fractions for households in each enumeration area (cluster) therefore included the 
first stage probability of selection of the enumeration area in that particular sampling stratum and the second stage probability of selection of a household in the sample enumeration area (cluster).

\section{Adjustment of sample weights for non-response}

A second component in the calculation of sample weights takes into account the level of non-response for the household interviews. It is rarely the case that all of the information desired is obtained from all sampled units in surveys. For instance, some households may provide no data at all. If there are any systematic differences between the respondents and nonrespondents, then estimates based solely on the respondents will be biased. The adjustment for household non-response is equal to the inverse value of:

$\mathrm{RR}_{\mathrm{h}}=$ Number of interviewed households in stratum $\mathrm{h} / \mathrm{Number}$ of occupied households listed in stratum $\mathrm{h}$

After the completion of fieldwork, response rates were calculated for each sampling stratum. These were used to adjust the sample weights calculated for each cluster. However, analysis of variance ANOVA confirmed that the response rates do not differ significantly between strata, but they differ significantly between dzongkhags, so the weighted response rates of dzongkhags was calculated.

The design weights for the households were calculated by multiplying the above factors for each enumeration area. These weights were then standardised (or normalised), one purpose of which is to make the weighted sum of the interviewed sample units equal the total sample size at the national level. Normalisation is performed by dividing the aforementioned design weights by the average design weight at the national level. The average design weight is calculated as the sum of the design weights divided by the unweighted total.

\section{Adjustment for individual weights}

In order to extrapolate the weighting towards individuals rather than households, an additional adjustment is made. Sample weights were appended to all data sets and analyses were performed by weighting each household or individual, depending upon the purpose of the analysis. 
Karma Ura, Sabina Alkire, Tshoki Zangmo \& Karma Wangdi

\section{Appendix 2. Subjective Questions}

\begin{tabular}{|c|c|c|c|c|c|}
\hline Domain & Indicators & Variable(s) & Survey Question & Response range & Sufficiency \\
\hline \multirow{18}{*}{$\begin{array}{l}\text { Psychologic } \\
\text { al wellbeing }\end{array}$} & \multirow{4}{*}{$\begin{array}{l}\text { Life } \\
\text { satisfaction }\end{array}$} & Health & How satisfied are you with your health? & \multirow{4}{*}{$\begin{array}{l}5 \text { (Low-worst)-25 (High } \\
\text { satisfaction) }\end{array}$} & \multirow{4}{*}{$20-25$} \\
\hline & & Standard of living & ...with your standard of living? & & \\
\hline & & Occupation & ...with your major occupation? & & \\
\hline & & Family relationship & ...with your family relationship? & & \\
\hline & \multirow{5}{*}{$\begin{array}{l}\text { Positive } \\
\text { emotions }\end{array}$} & Calmness & \multirow{5}{*}{$\begin{array}{l}\text { During the past few weeks, how often do } \\
\text { you experience__(Emotion)_? }\end{array}$} & \multirow{5}{*}{$\begin{array}{l}5 \text { (Low )-20 (High positive } \\
\text { emotion score) }\end{array}$} & \multirow{5}{*}{$\begin{array}{l}\text { 15-20 (Positive } \\
\text { emotion score) }\end{array}$} \\
\hline & & Compassion & & & \\
\hline & & Forgiveness & & & \\
\hline & & Contentment & & & \\
\hline & & Generosity & & & \\
\hline & \multirow{5}{*}{$\begin{array}{l}\text { Negative } \\
\text { emotions }\end{array}$} & Selfishness & \multirow{5}{*}{$\begin{array}{l}\text { During the past few weeks, how often do } \\
\text { you experience __(Emotion)_? }\end{array}$} & \multirow{5}{*}{$\begin{array}{l}5 \text { (Low)-20 (High negative } \\
\text { emotion score) }\end{array}$} & \multirow{5}{*}{$\begin{array}{l}\text { 15-20 (Negative } \\
\text { emotion score) }\end{array}$} \\
\hline & & Jealousy & & & \\
\hline & & Fear & & & \\
\hline & & Worry & & & \\
\hline & & Anger & & & \\
\hline & \multirow{4}{*}{ Spirituality } & Spirituality & How spiritual do you consider yourself? & 1 (Not at all) -4 (Very spiritual) & 4 (Very spiritual) \\
\hline & & Karma & $\begin{array}{l}\text { Do you consider Karma in the course of } \\
\text { your daily life? }\end{array}$ & 1 (Not at all)-4 (Always) & 4 (Always) \\
\hline & & Prayer recitation & How often do you recite prayers? & 1 (Not at all)- 4 (Regularly) & 4 (Regularly) \\
\hline & & Meditation & How often do you meditate? & 1 (Not at all) - 4 (Regularly) & $\begin{array}{l}3 \text { (Occasionally) or } \\
4 \text { (Regularly) }\end{array}$ \\
\hline Health & $\begin{array}{l}\text { Self-reported } \\
\text { health }\end{array}$ & Self-reported health status & $\begin{array}{l}\text { In general, would you say your health } \\
\text { is... }\end{array}$ & 1 (Very poor) -5 (Excellent) & $\begin{array}{l}4 \text { (Good) or } 5 \\
\text { (Excellent) }\end{array}$ \\
\hline \multirow{2}{*}{$\begin{array}{l}\text { Good } \\
\text { governance }\end{array}$} & \multirow{2}{*}{$\begin{array}{l}\text { Governance } \\
\text { performance }\end{array}$} & Creating jobs & $\begin{array}{l}\text { Rate the performance of government in } \\
\text { creating jobs? }\end{array}$ & \multirow{2}{*}{$\begin{array}{l}7 \text { (Low institutional } \\
\text { performance score) } \\
-35 \text { (High institutional } \\
\text { performance) }\end{array}$} & \multirow{2}{*}{$\begin{array}{l}28-25 \\
\text { (Institutional } \\
\text { performance score }\end{array}$} \\
\hline & & $\begin{array}{l}\text { Reducing gap between rich and } \\
\text { poor }\end{array}$ & in reducing gap between rich and poor? & & \\
\hline
\end{tabular}


An Extensive Analysis of GNH Index

\begin{tabular}{|c|c|c|c|c|c|}
\hline & & Fighting corruption & in fighting corruption? & & \\
\hline & & $\begin{array}{l}\text { Preserving culture and } \\
\text { traditions }\end{array}$ & in preserving culture and traditions? & & \\
\hline & & Protecting environment & in protecting environment? & & \\
\hline & & Providing educational needs & in providing educational needs? & & \\
\hline & & Improving health services & in improving health services? & & \\
\hline & \multirow{7}{*}{$\begin{array}{l}\text { Fundamental } \\
\text { rights }\end{array}$} & Freedom of speech & $\begin{array}{l}\text { Do you feel that you have a right to the } \\
\text { freedom of speech and opinion? }\end{array}$ & \multirow{7}{*}{1 (No)-2 (Yes) } & \multirow{7}{*}{2 (Yes) } \\
\hline & & Vote & ...have a right to vote? & & \\
\hline & & Join political party & $\begin{array}{l}\text {...have a right to join political party of } \\
\text { your choice? }\end{array}$ & & \\
\hline & & Form tshogpa & ...have a right to form tshogpa? & & \\
\hline & & $\begin{array}{l}\text { Equal access to join public } \\
\text { service }\end{array}$ & $\begin{array}{l}\text {..have a right to equal access and } \\
\text { opportunity to join public service? }\end{array}$ & & \\
\hline & & To equal pay for equal work & $\begin{array}{l}\text {...have a right to equal pay for work of } \\
\text { equal value? }\end{array}$ & & \\
\hline & & Free from discrimination & $\begin{array}{l}\text {..have a right to the freedom from } \\
\text { discrimination? }\end{array}$ & & \\
\hline \multirow{9}{*}{$\begin{array}{l}\text { Ecological } \\
\text { diversity } \\
\text { and } \\
\text { resilience }\end{array}$} & $\begin{array}{l}\text { Responsibility } \\
\text { towards } \\
\text { environment }\end{array}$ & $\begin{array}{l}\text { Feelings of responsibility } \\
\text { towards environment }\end{array}$ & $\begin{array}{l}\text { Do you feel responsible for conserving } \\
\text { the natural environment? }\end{array}$ & $\begin{array}{l}1 \text { (Not at all responsible)-4 } \\
\text { (Highly responsible) }\end{array}$ & $\begin{array}{l}4 \text { (Highly } \\
\text { responsible) }\end{array}$ \\
\hline & \multirow{8}{*}{$\begin{array}{l}\text { Ecological } \\
\text { issues }\end{array}$} & Pollution of rivers and streams & \multirow{8}{*}{$\begin{array}{l}\text { Is _ } \quad \text { (potential issue }) \quad \text { an } \\
\text { environmental issue of concern in your } \\
\text { community? }\end{array}$} & \multirow{8}{*}{$\begin{array}{l}1 \text { (Major concern) }-4 \text { (Not a } \\
\text { concern) }\end{array}$} & \multirow{8}{*}{$\begin{array}{l}4 \text { (Not a concern) } \\
\text { or } 3 \text { (Minor } \\
\text { concern) or } 2 \\
\text { (Some concern) in } \\
\text { at least } 6 \text { ecological } \\
\text { issues }\end{array}$} \\
\hline & & Air pollution & & & \\
\hline & & Noise pollution & & & \\
\hline & & Absence of waste disposal sites & & & \\
\hline & & Littering & & & \\
\hline & & Landslides & & & \\
\hline & & Soil erosion & & & \\
\hline & & Floods & & & \\
\hline
\end{tabular}


Karma Ura, Sabina Alkire, Tshoki Zangmo \& Karma Wangdi

\section{Appendix 3. Self-Report Questions}

\begin{tabular}{|c|c|c|c|c|c|}
\hline Domain & Indicators & Variable(s) & Survey Question & Response range & Sufficiency \\
\hline \multirow{3}{*}{$\begin{array}{l}\text { Psychological } \\
\text { wellbeing }\end{array}$} & \multirow{3}{*}{ Spirituality } & Karma & $\begin{array}{l}\text { Do you consider Karma in the course of } \\
\text { your daily life? }\end{array}$ & $\begin{array}{l}1 \text { (Not at all })-4 \\
\text { (Always) }\end{array}$ & 4 (Always) \\
\hline & & Prayer recitation & How often do you recite prayers? & $\begin{array}{l}1 \text { (Not at all) }-4 \\
\text { (Regularly) }\end{array}$ & 4 (Regularly) \\
\hline & & Meditation & How often do you meditate? & $\begin{array}{l}1 \text { (Not at all) }-4 \\
\text { (Regularly) }\end{array}$ & $\begin{array}{l}3 \text { (Occasionally) or } 4 \\
\text { (Regularly) }\end{array}$ \\
\hline \multirow{10}{*}{ Education } & \multirow{5}{*}{ Knowledge } & $\begin{array}{l}\text { Local legend and folk } \\
\text { stories }\end{array}$ & $\begin{array}{l}\text { How would you rate your knowledge and } \\
\text { understanding of local legend and folk } \\
\text { stories? }\end{array}$ & \multirow{5}{*}{$\begin{array}{l}5 \text { (Low knowledge } \\
\text { score)-25 (High } \\
\text { knowledge score) }\end{array}$} & \multirow{5}{*}{$\begin{array}{l}\text { 19-25 (Knowledge } \\
\text { score) }\end{array}$} \\
\hline & & Local tshechus & $\begin{array}{l}\text { How would you rate your knowledge and } \\
\text { understanding of local tshechus? }\end{array}$ & & \\
\hline & & Traditional songs & $\begin{array}{l}\text { How would you rate your knowledge and } \\
\text { understanding of traditional songs? }\end{array}$ & & \\
\hline & & The Constitution & $\begin{array}{l}\text { How would you rate your knowledge and } \\
\text { understanding of the Constitution? }\end{array}$ & & \\
\hline & & $\begin{array}{l}\mathrm{HIV} / \mathrm{AIDS} \\
\text { transmission }\end{array}$ & $\begin{array}{l}\text { How would you rate your knowledge on } \\
\text { how HIV/AIDS is transmitted? }\end{array}$ & & \\
\hline & \multirow{5}{*}{ Value } & Killing & Is killing justifiable? & \multirow{5}{*}{$\begin{array}{l}5 \text { (Low value score) }-15 \\
\text { (High value score) }\end{array}$} & \multirow{5}{*}{ 14-15 (Value score) } \\
\hline & & Stealing & Is stealing justifiable? & & \\
\hline & & Lying & Is lying justifiable? & & \\
\hline & & Disharmony & $\begin{array}{l}\text { Is creating harmony in human relations } \\
\text { justifiable? }\end{array}$ & & \\
\hline & & Sexual misconduct & Is sexual misconduct justifiable? & & \\
\hline \multirow{2}{*}{$\begin{array}{l}\text { Community } \\
\text { vitality }\end{array}$} & \multirow{2}{*}{$\begin{array}{l}\text { Community } \\
\text { relationship }\end{array}$} & Sense of belonging & $\begin{array}{l}\text { How would you describe your sense of } \\
\text { belonging to your local community? }\end{array}$ & $\begin{array}{l}1 \text { (Weak)-3 (Very } \\
\text { strong) }\end{array}$ & 3 (Very strong) \\
\hline & & Trust in neighbors & How much do you trust your neighbors? & $\begin{array}{l}1 \text { (Trust none of } \\
\text { them) }-4 \text { (Trust most }\end{array}$ & $\begin{array}{l}4 \text { (Trust most of } \\
\text { them) }\end{array}$ \\
\hline
\end{tabular}


An Extensive Analysis of GNH Index

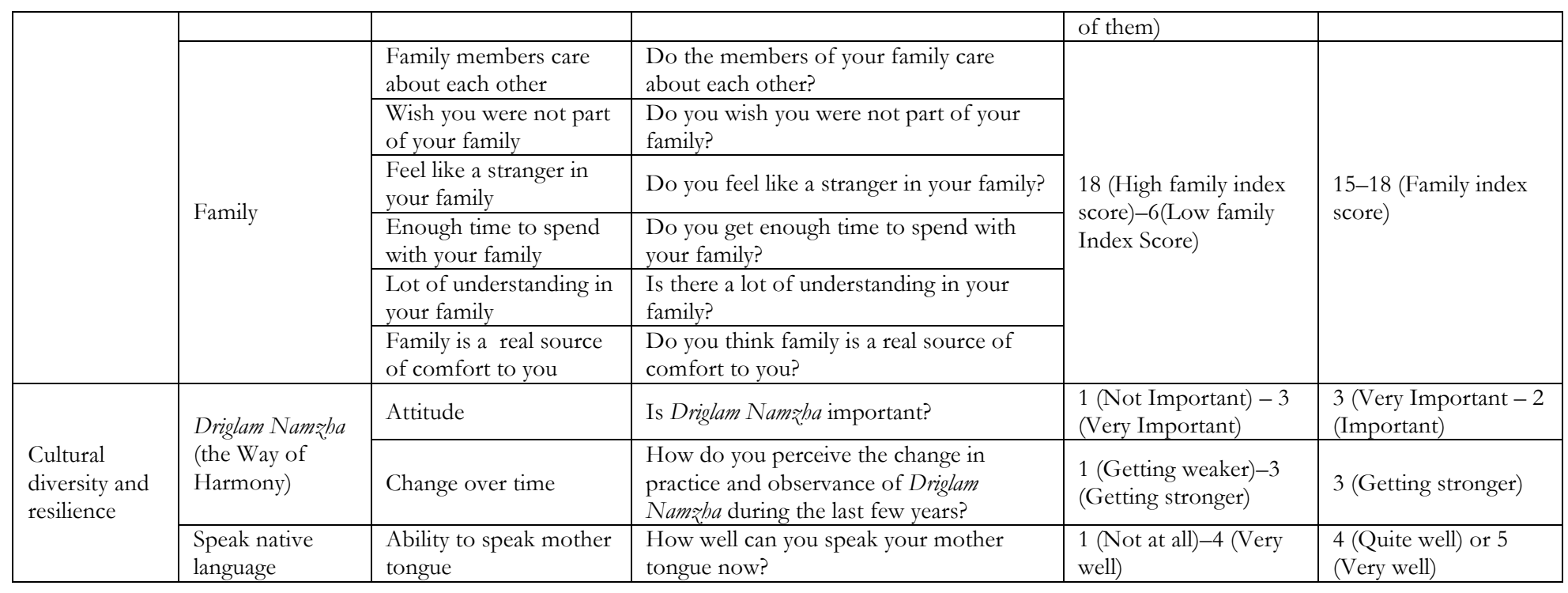




\section{Appendix 4. Fieldwork Cases}

Two focus group discussions were held as case studies in order to obtain a better understanding of people's perceptions of Gross National Happiness and its components. There were two central purposes of the focus group discussions. The first was to explore the main factors that participants believed were responsible for a decline or improvement in people's wellbeing in their village. The second was to discuss thresholds or cutoffs for some of the indicators. These exercises were undertaken as a means to develop more effective GNH indicators. It also allowed us to gather direct insights on their conceptual value judgments. The focus group discussions were carried out in two villages: Samdrupgang village in Rubesa gewog of Wangdue Phodrang and the other in Laptsakha village in the Talo gewog of Punakha. There were about 10 to 16 participants in each group with an almost equal number of men and women. On average there were more elderly participants and fewer young people.

The sessions started with a casual discussion of some of the major changes happening in their communities. This was done to explore the extent to which various interventions contributed to improvements in people's wellbeing. Discussions of the causes of decline and improvement provided an initial picture of the pressures and opportunities facing people. Then the participants were asked to reflect on the most important factor influencing their happiness or wellbeing and to justify those factors. Respondents were asked what images come to mind when the phrase 'wellbeing' is mentioned and why those associations are made. Based on the respondents' spontaneous suggestions and reactions, a definitive list of factors was agreed upon within each group. This process allowed an initial open brainstorming discussion to take place followed by a consensusfinding exercise where the most important causes of decline or improvement of one's wellbeing were chosen by the group.

Every household has its conception of 'good life,' and it is related to specific aspects of the lives of each of the household members. For some participants it can also be reduced down to an individual conception of wellbeing. So, depending on the diverse needs of the people, different factors played dominant role. Generally, there were about six factors that were included in the groups' definitive lists - health, relationship, spiritualty (contentment), financial security, education and job satisfaction. With spirituality, participants took special note of the value of contentment. Similarly, the economic aspect was perceived more along the lines of financial security. 
Next, the nine domains of GNH were introduced to the group and a series of ranking or prioritization exercises were undertaken to gain some insight into how easy it was for participants to differentiate between the domains. The exercise required all the domains to be put in order of their relative importance.

In both the focus groups, health and education were seen as the most important. Living standards ranked second.

\begin{tabular}{|l|l|l|l|}
\hline Group & \multicolumn{1}{|c|}{$\mathbf{1}$} & \multicolumn{1}{|c|}{$\mathbf{2}$} & \multicolumn{1}{c|}{$\mathbf{3}$} \\
\hline Rubesa & $\begin{array}{l}\text { Health, Education, } \\
\text { Psychological } \\
\text { wellbeing }\end{array}$ & $\begin{array}{l}\text { Living standards, } \\
\text { Good governance, } \\
\text { Culture }\end{array}$ & $\begin{array}{l}\text { Community vitality, } \\
\text { Ecological diversity and } \\
\text { resilience }\end{array}$ \\
\hline Laptsakha & $\begin{array}{l}\text { Health, Education, } \\
\text { Psychological } \\
\text { wellbeing }\end{array}$ & $\begin{array}{l}\text { Living standards, } \\
\text { Culture, Time use, } \\
\text { Community vitality }\end{array}$ & $\begin{array}{l}\text { Good governance, } \\
\text { Ecological diversity and } \\
\text { resilience }\end{array}$ \\
\hline
\end{tabular}

The present situation of participants seems to have a lot of influence on the way different domains were ranked. If participants were experiencing problems within one of the domains, that domain was given a higher priority. For example, some participants who were coping with relationship issues, prioritized family while elderly participants stressed spirituality and contentment. Participants with money problems talked more about money and were more inclined to put living standards in first place. The same happened with health, although people without health problems also considered it a top issue.

Lastly, participants were asked about their perspectives on the thresholds of some of the indicators. For example: How much should one donate in a year? How much sleep is necessary? How many years of education are sufficient? How much time should be spent working?

Although participants pointed out the difficulty in setting a sensitive threshold with respect to the diverse backgrounds of individuals, various suggestions and comments were made based on their experiences of what would be enough. In terms of sleeping hours, the consensus was 6 to 7 hours while the threshold for years of education was thought to be at least 10 standard. With indicators like donations, land size or community participation, participants emphasised that these indicators depended entirely on the economic and social conditions of households and therefore must be considered on a case-by-case basis. Nevertheless, some of the participants also stated that for an average household size of five, about five acres would be sufficient. Likewise, for donations about $5 \%$ to $10 \%$ of the income would be affordable. This discussion of thresholds, rather the brainstorming session, provided us with perspectives and 
priorities, thus enabling us to identify certain issues to be considered when selecting thresholds.

In conclusion, the focus group discussions provided the team with information about people's perceptions of wellbeing and its determinants. It drew attention to their interconnectedness. The discussions also seem to suggest that setting thresholds would be more of an educated guess than a standard method and therefore require constant review and adaption in the years to come. 


\section{Appendix 5. The 33 GNH Indicators: Their Construction and Justification}

In order to measure the nine domains of GNH, 33 indicators have been selected according to five different criteria. First of all the indicators have to reflect the normative values of GNH which have been articulated in official documents such as the National Development Plan and in statements by His Majesty the King, the Prime Minister and other ministers. They also reflect the normative values which are embedded in the culture and traditions of Bhutan. The second criterion for the indicators relates to their statistical properties: each indicator was analysed extensively to ensure robustness. Third, the indicators were chosen such that they would accurately reflect how happiness is increasing or evolving in different regions over time and among different groups accurately. Fourth the indicators had to be relevant for public action - although government policy is by no means the only way of increasing GNH. Many domains of $\mathrm{GNH}$ can be facilitated by appropriate government policies and by government policies that create incentives for business, NGOs and citizens to support GNH in its many dimensions. And lastly, the indicators have to be understandable as far as possible by citizens. They have to reflect and relate to people's own experiences in their own lives, so that the GNH Index would not only be a policy tool but would also be something that people could use to imagine the many different ways of being happy in the Bhutanese context.

There are four indicators in every domain, except time use, which has two (sleep and work), and living standards, which has three. This appendix provides a detailed explanation of the indicators that have been included in the index, with particular emphasis on their construction, their justification, and their statistical properties.

\section{Psychological Wellbeing}

Psychological wellbeing is an intrinsically valuable and desired state of being. (Diener et al. 1997) categorise indicators of psychological wellbeing according to reflective or affective elements. Reflective indicators provide an appraisal of how satisfied people are in various aspects of their lives while the affective indicators provide a hedonic evaluation guided by emotions and feelings such as the frequency with which people experience various moods in reaction to their lives. 
The Sarkozy Report ${ }^{28}$ (Stiglitz, Sen and Fitoussi, 2009a, p. 44) emphasises the importance of using diverse wellbeing indicators. It states: '...different aspects (cognitive evaluations of one's life, happiness, satisfaction, positive emotions such as joy and pride, and negative emotions such as pain and worry)... should be measured separately to derive a more comprehensive appreciation of people's lives.' Many other studies support the use of multiple subjective measures of wellbeing (McGillivray and Clarke 2006; van Hoorn 2007; Samman 2007).

Following this, the GNH Index uses indicators of satisfaction and of emotional wellbeing. An additional aspect of spirituality has also been included in the domain. From a GNH perspective, spirituality is fundamental for one's wellbeing and happiness, and so continuous efforts must be made by individuals and societies to develop their full spiritual potential. Spirituality also represents a vital part of identity since it is deeply rooted in Bhutan's tradition and culture. Therefore, psychological wellbeing has been expressed in terms of four major components: life satisfaction, positive emotions, negative emotions and spirituality.

The four indicators use subjective and self-report data to proxy a person's psychological wellbeing. Like all subjective indicators, they require care in interpretation. This is particularly the case for the GNH Index, because each response is interpreted as reflecting a particular individual's general psychological wellbeing state for the survey year. Yet a person's particular responses may be an imperfect proxy of their psychological wellbeing. For example, the respondents will have distinct personality types and different reference groups in mind for their responses. There may be mood and framing effects, as well as influences from the dynamics with the particular enumerator. There may also be a hesitation to answer certain questions accurately: to a deeply spiritual person it might seem presumptuous or culturally inappropriate to claim that they consider themselves 'highly spiritual'. All of these will create some well-known distortions. In this domain, we have tried to mute at least some distortions by creating valid indices using multiple questions for three of the indicators. The results by groups replicate well-known findings in the literature - for example, showing that employment and relationships are key for psychological

\footnotetext{
28 The report narrates an extensive review of the composition of subjective wellbeing into two major components: first, the evaluation of a person's life as a whole or of various domains and second, the measurement of the actual feelings. Both the components are reflected in the psychological wellbeing domain of GNH and were computed separately. The report states, 'that these measures provide information about the determinants of quality of life at the level of each person. These determinants include both features of the environment where people live and their individual conditions, and they vary depending on the aspect considered.' Further, it highlights that these subjective measures provide information beyond what is being given by income.
} 
wellbeing. ${ }^{29}$ Further, all indices have been constructed after performing certain validity tests such as principal component analysis (PCA) and factor analysis (FA).

The text below explains each of the components of the psychological wellbeing domain. It has three indicators composed of four sub-indices, as positive and negative emotions are entered separately.

\section{Life satisfaction}

Many have advocated that quality of life be measured using indicators that reflect evaluative assessments of satisfaction with life overall (Kahneman and Krueger 2006; Stiglitz, Sen and Fitoussi 2009b; Deaton 2010) rather than hedonic measures of fleeting moments of joy or passing moods. Veenhoven (1991) has argued that making an overall judgment about one's life implies a cognitive, intellectual activity and requires the assessment of past experiences and estimation of future experiences. It was found that a global measure of life satisfaction should provide reliable information in many policy contexts (DEFRA 2011). ${ }^{30}$ Therefore, in the case of the GNH survey, an overview question was implemented, which is how satisfied are you with the quality of your life?'

Enumerators observed, however, that Bhutanese respondents had distinct reference points in mind whilst responding, suggesting that at the individual level the responses were not necessarily reflective of overall subjective wellbeing. Pragmatic inferences about the intended meaning of a question are at the heart of many context effects in survey measurement (Schwartz 1992). Although single-item scales may be tested to ascertain whether they have adequate convergent validity (that is, scales correlate well with other similar measures), only multiple scales allow for assessment of internal consistency, as well as for the identification of errors associated with wording (translation). Diener (1984) found that multi-item scales have demonstrated greater reliability and validity than single-item scales. Stock

\footnotetext{
${ }^{29}$ Linear regression with some of the indicators of the psychological wellbeing domain was carried out with demographic characters such as gender, occupational level, region, age, family relationships etc. The life satisfaction indicator shows a significant association with family relationship. The higher the life satisfaction score, the higher the family relationship score (coef $=.366, \mathrm{P}>0.000$, after observing demographic characters as constant). Unemployed people show a lower life satisfaction score as compared to civil servants (coeff $=-.88, \mathrm{P}=0.001)$. Positive emotions indicators also showed similar results with respect to relationship and occupational status.

${ }^{30}$ Life satisfaction is used as a headline measure for wellbeing in the Sustainable Development Indicator developed by DEFRA www.defra.gov.uk/sustainable/government/progress/national/68a.htm
} 
and Okun (1982) presented substantial evidence for the construct validity of life satisfaction. Further, it has been observed that a single-item question is often susceptible to bias because it restricts responses to a specific reference point of view while the multi-item scale uses a wider range of information with more specificity.

For these reasons, a multi-item life satisfaction indicator was developed to test the overall life satisfaction of an individual. The survey included questions on satisfaction with life in particular domains. It was important to keep the satisfaction questions as simple and as interpretable as possible due to illiteracy issues in the country. These questions had been pretested and respondents rarely failed to respond to such questions indicating low reporting bias; this was corroborated by enumerator observation that the questions were comprehensible and easy to answer.

The satisfaction indicator combines individuals' subjective assessments of their contentment levels with respect to health, occupation, family, standard of living and work-life balance. ${ }^{31}$ The respondents were asked to say how satisfied or dissatisfied they were in these five areas on a fivepoint Likert scale $(1=$ very dissatisfied, $5=$ very satisfied $)$.

The validity of the aggregate indicator was tested using PCA. The number of factors to be extracted was determined by an inspection of the scree plot of eigenvalues. Using this criterion, the items appear to hold in a unified factor (factor loadings above .47) showing that there is coherence to life satisfaction. ${ }^{32}$ Reliability analysis showed satisfactory results (Cronbach's alpha of 0.7). ${ }^{33}$ These results indicate that the five-item satisfaction questionnaire could be collapsed into a single uni-dimensional indicator. In this case, the variables were aggregated using equal weights because each domain seemed relatively equal in importance across different phases of the life cycle. The life satisfaction indicator score

\footnotetext{
31 A five item Likert scale was used rather than the single item question on life satisfaction because dissatisfaction in life is usually due to dissatisfaction in any of multiple areas of life. One of these areas can pull down the satisfaction level (Diener, 2006).

32 Similar analysis is presented Review of the Satisfaction with Life Scale by Pavot and Diener (1993).

${ }^{33}$ For a standard of reliability, DeVellis (1991) stated 'below 0.60, unacceptable: between 0.60 and 0.65 , undesirable; between, 0.65 and 0.70 minimally acceptable; between 0.70 and 0.80 , respectable; between 0.80 and 0.90 , very good; much above 0.90 , one should consider shortening the scale' (p. 85). The reliability coefficient for evaluation uses would be at least 0.70 and preferably 0.80 for groups and at least 0.90 for individual decision making (Nunnally, 1978). Clearly, there are varying thresholds for the test of reliability. In the 2010 GNH Index we have considered $\geq 0.8$ to be good, $0.7-0.79$ to be satisfactory, $0.6-.69$ to be acceptable and 0.5-0.49 to be inadequate.
} 
ranged from 5 (low satisfaction) to 25 (high satisfaction). The mean score was $20(\mathrm{SD}=2.5)$. A positive correlation was observed with the happiness question $(\mathrm{r}=.39)$ and the overall satisfaction with life score $(\mathrm{r}=.42)$, and a negative correlation with the 12 -item General Health Questionnaire (GHQ-12) which detects minor psychological distress $(r=-.44.){ }^{34}$ These associations further confirm, to a limited extent, the construct validity of the life satisfaction indicator.

The sufficiency threshold for the life satisfaction score is set at 19. It implies that a person should rate themselves as either 'satisfied' or 'very satisfied' in at least four and 'neither satisfied nor dissatisfied' in the other, or 'very satisfied' in four and 'dissatisfied' in one. Clearly, there can be other combinations, but the idea is that people should have a score of 19 or above to be categorised as being in a happy condition. Note that the threshold provides room for dissatisfaction to occur in at least one component, implying the importance of the overall score rather than individual scores. Usually, respondents report similar satisfaction across components, for instance if someone has reported satisfaction in two components then it is more likely that they report the same in the other three as well. For instance, $58 \%$ of the respondents have rated 'satisfied' or 'very satisfied' in all five components.

Other options using a relative threshold of sufficiency such as the mean and median have been explored empirically and indeed in this particular case both take the value of 20. However normatively, a GNH view (like that of Layard and others) encourages people to assess their happiness in an absolute sense and discourages the use of relative or positional references for happiness, as the comparative approach can never generate very widespread happiness. Thus, the threshold of 19 has been applied.

When the sufficiency threshold of 19 is applied, about $83 \%$ of respondents are classified as happy. However because of the response scale and low levels of inequality, the percentage is very sensitive to changes in the sufficiency threshold. Threshold values of 20 and 18 classified $72 \%$ and $89.6 \%$ of respondents as happy in life satisfaction respectively. Setting a threshold at 20 is perhaps too severe since there is no freedom for a lower rating at all while 18 perhaps ensures too much flexibility by allowing dissatisfaction in two components. So, the

\footnotetext{
34 A polychoric correlation between the life satisfaction indicator, happiness level (0-10 point scale) and life quality (1-5 point scale) was observed. Life satisfaction showed a positive correlation with life quality $(\mathrm{r}=.42)$ and happiness level $(\mathrm{r}=0.4)$. A Spearman correlation between life satisfaction and the GHQ-12 indicator of mental health resulted in significant negative correlation $(\mathrm{r}=1.44)$
} 
sufficiency threshold of 19 was chosen and $83 \%$ were sufficient in the life satisfaction indicator.

\section{Emotional Balance (Positive and Negative Emotions)}

Emotions strongly influence people's thoughts and actions and thereby deeply influence one's wellbeing. ${ }^{35}$ It is vital for us to understand and distinguish the type of emotional experiences that are beneficial for one's wellbeing from the ones which are harmful. It can also be useful to recognize that a radical transformation of emotions can occur by mindfulness, introspection and a healthy external environment.

In general, psychologists usually do not distinguish between beneficial and harmful emotions. Those who take an evolutionary view of emotions have proposed that emotions were adaptive over the history of the species and remain adaptive today (Ekman 1992; Cosmides and Tooby 2000). Even those who categorise emotions into positive and negative do not propose that all negative emotions are harmful to the individual or to others (Watson, Clark and Tellegen 1988). Most understand all emotions to be an aid for survival and necessary for the full range of human experience. In contrast, from the Buddhist viewpoint prevalent in Bhutan, it is crucial to develop positive emotions while reducing the force of negative emotions, in order to increase one's happiness and wellbeing. The GNH Index thus reflects this position.

There are many studies that stress the benefits of positive emotions. Positive emotions are often associated with situations that present opportunities rather than threats, and associated with a strategy of approaching rather than avoiding problems (Fredrickson 2000; Nesse 2004). Studies also suggest that positive and negative states selectively trigger different information processing units in human brain. Positive affect uses internalised strategies invoking knowledge structures known as assimilative thinking, while negative emotions encourage a focus on external, environmental information which Fiedler and Bless (2001) called accommodative thinking. Some studies confirm the beneficial effect of positive emotions on health. It has been proposed that one of the factors affecting the relationship between positive emotions and health is the functioning of the immune system (Davidson et al. 2003).

In measurement aspects, though reliability has been tested, emotional reporting is often biased towards the most recent or most intense period of experience (Kahneman, Fredrickson, Schreiber and Redelmeier 1993;

${ }^{35}$ See for example, Ekman et al. (2005) 
Rosenberg and Ekman 1994). A common measure of emotional experience is the ten-item mood scale that comprises the PANAS (Positive Affect and Negative Affect Schedule) developed by Watson, Clark and Tellegen (1988). PANAS demonstrates that stability is more precise over one to two month time period than when used with short-term instructions. STEM (State Trait Emotion Measure) is another recently constructed scale composed of five positive and five negative emotions (Levine and Xu 2005).

Although a range of indicators exist to assess emotions, many indicators were observed to be difficult to implement in the Bhutanese context due to difficulties in translation. We understand that there are significant differences in the emotional development in people depending on the socio-cultural circumstances, and there are societies in which certain kinds of emotions are more publicly expressed than others Therefore, for Bhutan a list of emotional experiences was drawn up based on the dominant social ethos while also keeping in mind the degree of expressiveness in the culture. Ten self-reported emotion items were selected. Positive emotions, or non-disturbing emotions, such as compassion, generosity, forgiveness, contentment and calmness were included while selfishness, jealousy, anger, fear and worry were used to represent negative emotions. In the Buddhist perspective, the negative emotions may be more accurately called disturbing emotions during which people cannot experience with much clarity and that might lead often to the formation of poor intentions. For both sets of emotions the respondents were asked to rate the extent to which they have experienced them during the past few weeks with reference to a four-point scale. ${ }^{36}$ The scale ranges are: 1 'never', 2 'rarely', 3 'sometimes', and 4 'often'.

Next, we created an indicator related to the emotions. We separated this into indices of positive and negative emotions, following the literature which suggests that positive and negative emotions are not polar opposites and so should be considered separately (Kahneman and Krueger). ${ }^{37}$ In the

\footnotetext{
36 A number of different time frames have been used in various studies (Green, Goldman and Salovey 1993; Watson, Clark and Tellegen 1988 ; Watson and Tellegen 1999).The use of a 'few weeks' reference period is not ideal; ideally we would have information on average emotional experiences throughout the past year. But this may be too difficult to recall accurately. The GNH emotional indices will be partly inaccurate as a reflection of annual emotional states for at the individual level because 'the past few weeks' will not have been representative for all respondents. However they were the best that could be constructed from the available data.

37 A model has been developed by J.A.Russell and J.M. Carroll (1999) that defines happiness and sadness as polar opposites. On the other hand, there are studies that disagree
} 
initial phase of scale construction using PCA, both negative and positive emotions were included to test the latent constructs. Initial factor analysis generated three factors; one positive emotion component (five items) and two negative emotion components (two items and three items). The items when tested for internal consistency showed a satisfactory Cronbach's alpha score (positive emotions alpha score $=.74$ ). There is a significant difference between the frequencies of positive emotions felt by people who have prayed more or less. Likewise, respondents scoring high on consideration of karma have a higher frequency of positive emotions. ${ }^{38}$ So, the positive emotion indicator does seem to be accurate in terms of its intended measurement. The two negative emotion components had Cronbach's alpha scores of .66 and .78. The mean score for the positive emotion was $14.87(\mathrm{SD}=3)$. The mean score for two item negative emotions score was $7.4(\mathrm{SD}=1.1)$ and three item negative emotion score was $8.6(\mathrm{SD}=2.4)$. The negative emotion score has a positive correlation with GHQ-12 $(r=.4, r=.13){ }^{39}$ The correlation between negative emotions and positive emotions is low, which follows experiences in other locations. ${ }^{40}$

The positive emotion indicator score runs from 5 to 20 . A score of 20 reflects a very high positive emotional experience while 5 indicates a very low incidence of positive emotion. Ideally, from a GNH perspective, the threshold might be set at 20 since the goal is to develop a society where there is an expression of high positive emotions. ${ }^{41}$ But due to the possible variations in the responses either influenced by typical circumstances or just random responding (Larsen et al. 2001), it seemed more reasonable to set a lower threshold of 15, which would allow for response errors. It must be noted for future surveys, that there was a lack of distinct clarity between

over this relationship and propose that the positive and negative can co-occur at some levels (Diener and Iran-Nejad 1986, Watson et al 1999).

38 This has been assessed using a one-way ANOVA test. With respect to prayer recitation, respondents who had higher frequencies also reported a higher prevalence of positive emotions $(\mathrm{P}<0.05)$. Likewise, consideration of karma showed a significant difference in means of people who had higher and lower positive emotions $(\mathrm{P}<0.05)$.

39 A Spearman correlation resulted in a positive correlation between GHQ-12 and negative emotion scores $(r=.4$ and $r=.13)$.

40 This low correlation is supported by studies that show positive and negative emotions to be independent, and the conclusion was that emotional experience could be conceptualised as two components (Tomkins 1981; Bradburn 1969). Nevertheless, there are issues of low reliability, item sampling, type of response format and time frame covered suggesting that the scale itself might account for the observed independence of the components (Watson et al 1988; Diener and Emmons 1984; Egloff 1998).

41 When the threshold is set high at 20 in which all emotions have to be rated as 'often', almost everyone is identified as having insufficient psychological wellbeing ( $92 \%$ of respondents). 
response ranges. Setting a sufficiency threshold of 15 would mean that the frequency of feelings of positive emotions has to be either 'often' or 'sometimes' in at least four emotions. ${ }^{42}$ The threshold identifies $58.8 \%$ as being happy in positive emotions.

The negative emotion indicator scores also runs from 5 to 20 (from low to high incidence of positive or negative emotions). The negative emotion indicator consists of two components of sub-indices. The emotions included are selfishness and jealousy in one sub-index and anger, fear and worry in the other sub-index. As mentioned before, if one takes one perspective - namely that negative emotions are to be gradually overcome - ultimately it would be best to have a society with a low frequency of negative emotions. However the aim would clearly not be to repress negative emotions, so some negative emotions will be experienced. In general, the scores of 'often' are low. Anger has been reported as 'often' only by $5 \%$ of the respondents, $6 \%$ report they have been often worried and only $1 \%$ have rated that they 'often' experience jealousy and selfishness. However, within the range of 'sometimes' anger is felt by $43.4 \%$, fear is felt by $30 \%$ and worry by $35 \%$, while sensitive emotions like selfishness and jealousy still have low ratings, $4 \%$ and $5 \%$ respectively. These results suggest that either the respondents truly have low frequencies of negative emotions overall, or the conceptual difference between 'often' and 'sometimes' might not be very clear, or that selfcentred emotions, which are much maligned in Bhutanese society, such as selfishness and jealousy, might be under-reported in face-to-face interviews.

If a threshold of 15 is set, which means a respondent might report 'often' in any one of the negative emotions and report the rest as 'never', 'rarely' or 'sometimes', $91.4 \%$ are happy. On the other hand, if a threshold of 10 is applied - implying that all ratings would be 'never' or 'rarely' - then $46.5 \%$ are happy. In order to allow a rating of 'sometimes' in at least two of the emotions, a sufficiency threshold of 12 has been considered for negative emotions, with about $64.6 \%$ of the respondents identified as happy.

\section{Spirituality}

While spirituality is a concept globally acknowledged, there is no consensus on how to define or measure it. Spirituality can encompass belief in spiritual values like compassion, peace, and a sense of purpose and connectedness. Acts of compassion, altruism and selflessness are often characteristics associated with spirituality. In Bhutan, addressing the

42 The mean is $14.9(\mathrm{SD}=3.03)$ and median score is 15 as well. 
spiritual dimension of a person's life has been a traditional way of bringing the person's wellbeing to the forefront (Ura 2010a). Bhutan is a spiritual nation and the influence of spirituality is highly visible in the everyday lives of the population, in spiritual gatherings, and in the numerous spiritual landmarks such as sacred temples and monasteries, prayer flags and prayer wheels. These provide a platform for people to develop spiritual maturity. In the context of GNH, spirituality is intrinsic to development, since, in essence GNH is based on balancing material wants with spiritual needs. So for meaningful development to occur it is of the utmost importance that societies have some measures for inner spiritual growth along with peaceful environment that allow spiritual nourishment. If material growth undermines the spiritual framework of society and its values of compassion and integrity, then development has not occurred.

There have been numerous attempts to measure spirituality. For example Paloutzian and Ellison (1982) designed a 20-item, self-administered scale to measure spiritual wellbeing in both its religious (RWB) and existential (EWB) senses. The Spiritual Perspective Scale (Reed 1987 and Belcher et al. 1989) attempted to measure the extent to which spirituality pervades people's lives and their engagement level with spiritual activities. Shalom Schwartz (1992) sought to identify cross-culturally valid indicators of transcendence but could not. ${ }^{43}$ But, for a society like Bhutan spirituality indicators need to be culturally adapted and easy to understand. So, in order to make a wider range of responses possible, simplified spirituality indicators were developed.

The spirituality indicator is based on four questions. A self-reported spirituality level describes the person's judgement of his or her own position on the spirituality continuum. The question of the consideration of karma asked people to what extent they take into account their own volitional impulses and actions as having moral consequences in future just as they did on the present. Measures of social engagements are dealt in both community vitality and time use domains. Here, indicators of sacred activities were limited to praying and meditation as two separate events although these activities are not mutually exclusive.

Note that the indicators allow for considerable variation in specific religious beliefs and habits. While Bhutan's culture is pervaded by Buddhism and the majority of Bhutanese self-identify as Buddhist, there is a significant Hindu population, as well other religious minorities including

43 Shalom Schwartz $(1992,2002)$ used his Schwartz Value Inventory (SVI) with a wide survey of over 60,000 people to identify common values that acted as 'guiding principles for one's life'. He identified ten 'value types' that gather multiple values into a single category. 
agnostic or atheist groups. All four questions are relevant to Hindu as well as Buddhists practitioners. The questions, however, might not adequately reflect the 'spirituality' of the newer cohort of agnostics or atheists who might experience transcendence through music, art or nature, but might not self-identify as spiritual. Also, a deeply spiritual person might be reluctant to classify themselves as 'very spiritual' - even though $50.4 \%$ of the sample did so.

All the four indicators run on a four-point scale of 'regularly' to 'not at all' except for the spirituality level which ranges from 'very spiritual' to 'not at all'. In terms of its empirical validity, a single factor consisting of three indicators of spirituality was extracted with loadings $>.34$. The meditation practice was observed to have a very low loading. The reliability of the overall indicator also decreased when meditation was included (Cronbach's alpha $=0.5)$. Without the indicator, it was .53 which indicates the spirituality indicator is better off without the meditation indicator. This is most probably due to its skewed distribution. About $80 \%$ never meditate and only $5 \%$ meditate regularly. ${ }^{44}$ Despite this, the indicator of meditation was included because of the importance of assessing trends over time. The government has recently initiated a school-based meditation curriculum, and meditation is attracting fast-growing interest among lay Bhutanese because of its ability to provide balance, positive emotions and mental clarity. The indicator had significant and expected correlations with other measures related to subjective wellbeing such as positive emotion score. ${ }^{45}$

The indicator sums the scores across the four questions. Scores range from 4 to 16 with 16 indicating a greater degree of spirituality. The threshold has been set at 12 which implies that at least three of the four indicators must be rated 'regularly' or 'occasionally' for individuals to be defined as happy. We understand that one of the key concerns in GNH is to maintain a vibrant spiritual culture and to track deteriorations of spirituality. Setting the highest possible threshold would enable assessments of both spiritual growth and deterioration over time. But due to the possible inaccuracies in responses mentioned above, the threshold was set at 12 instead. With a sufficiency threshold at 12,53\% are identified as having sufficient achievements in spirituality.

\footnotetext{
44 In Bhutan meditation is practiced mainly by the monks and nuns, and the GNH survey does incidentally include 25 monks or nuns but is not representative of monks and nuns (who make up about $3 \%$ of the population of Bhutan) because they are largely institutionalised, living in monasteries and what are called 'nunneries'.

45 Spearman correlation between positive emotion score and spirituality score indicated positive correlation $(\mathrm{r}=.3, \mathrm{P}<0.000)$
} 


\section{Health}

Health can be described as simply an absence of illness. However in Bhutan, health has always been associated with both physical health and mental health, a view reflected in the famous saying of 'luslu natsha med, semslus dugsngal med' [No illness in body and no stress in mind] (Wangdi 2010). Health is outcome of relational balance between mind and body, and between persons and the environment. Typically, an individual is said to be well only if heart-pain is absent from the body and sorrow is absent from the mind. This understanding conforms to the WHO's definition of health as 'a state of complete physical, mental and social wellbeing and not merely the absence of disease or infirmity'. While physical and mental health is important, a holistic approach towards health would focus on social circumstances, emotional states and spiritual aspects. Through a GNH lens, a combination of all would provide an individual with an ability to meet life's opportunities and challenges and maintain a level of functioning that has a positive influence on wellbeing (Ura 2008). Empirically, factor analysis revealed that mental health - which was categorised in the domain of psychological wellbeing in 2008 - loaded onto the factor having the other health variables. Hence it has been moved to the domain of health.

The social and material conditions for creating good health such as clean air or water or nurturing family relationships or community relationships have been incorporated into other domains. Similarly, emotional balance and spirituality have also been included in the psychological wellbeing domain. As a result, the health indicators in this domain describe only the physical and mental aspects of health performance. 46

Four indicators were chosen, including the number of healthy days in a month, self-reported health status, activity limitation and mental health. The first two indicators were developed by the US Centers for Disease Control and Prevention (CDC) to measure health-related quality of life (Hennessy et al. 1994). ${ }^{47}$ These assess an individual's physical health through four questions: 1) self-reported health status, 2) the number of

\footnotetext{
46 A plethora of health status measures have been proposed in the literature (Mahoney and Barthel 1965; Granger et al 1993; Bergner et al 1981; De Bruin et al 1992; Ware and Sherbourne 1992; McHorney et al. 1993; Kaplan and Bush 1982; EuroQoL Group 1990; Brazier et al. 1993; Elvik 1995; Feeney et al. 1995; Stein and Jessop 1990; McDowell and Newell, 1996; Ware 1995; McHorney 1999; Varni et al. 1999; CDC 2000). However, the choice of measure clearly depends on the validity and also the reliability of the measure with respect to the local and cultural context of the country.

47 The technical report titled Measuring Healthy Days published in year 2000 by the CDC provides a detailed description and validation of the set of measures for tracking the health of the population and health-related quality of life (HRQOL) in societies.
} 
days in which physical health was not good in the past month, 3) the number of days in which mental health was not good in the past month, and 4) the number of recent activity limitations due to poor physical or mental health (CDC 2000). However, in Bhutan the GNH survey dropped the fourth indicator and instead implemented the long-term disability indicator developed by the US Department of Health and Human Services in 2000. So, all in all there are four indicators of health: self-rated health status, healthy days, long-term disability and the mental health measure through the 12-item GHQ.

\section{Self-reported bealth status}

Self-rated health status has been one of the most frequently used health indicators in sociological health research (Jylhä 2009). Some studies using self-reported health portray it as a powerful predictor of subsequent mortality, even after controlling for individual characteristics, socioeconomic status, health behaviours and objective measures of health (Bake et al. 1999). Questions persist about how accurately this simple selfreported indicator proxies objective health and nutrition states, and the extent to which it is affected by 'adaptive preferences'. Easterlin (2003) points out that if self-reported health was affected by adaptation, then the life course trends in self-reported health should be flat and would also be flat if persons implicitly evaluate their health only by comparison with others of their age. He also found that adverse health changes have some negative effect on happiness and that there is a less than complete adaptation to deteriorating health (Easterlin 2003). Against that, Nobel Prize Laureate Amartya Sen documented how socially disadvantaged women in Bihar failed to perceive and report the presence of illness or health deficits, whereas highly educated women in Kerala had lower selfreported health despite having longer life expectancy and lower morbidity (Sen 2002). Others have confirmed his finding that people's self-reported health assessments are dependent on their aspirations and frame of reference. For example, Carol Graham (2010) shows how self-reported health levels in Kenya and the USA are roughly the same. Thus, selfreported health status may be an imperfect or misleading proxy of objective health status. Furthermore, trends in self-reported health may be difficult to interpret, because they can change either as a result of objective health status changes or because the frame of reference changes. It has also been argued that people's health perceptions are limited to their own knowledge and therefore are quite often inadequately informed (Kleinman 1995). Moreover, the claim that self-reported health is a valid and comparable proxy of overall health status is usually made for developed societies (Rahman, Menken and Kuhn 2004). 
Due to these issues, it would be preferable to use anthropometric and other more objective indicators of health. However the GNH survey did not provide these. Hence we subjected the self-reported health status to a number of exploratory tests. ${ }^{48}$ The analysis showed some demographic effects on self-reported health status, but they were by no means as strong as expected. It is noteworthy that we did not find higher self-reported rates of health in remote, rural and uneducated communities, suggesting that adaptive preferences may not distort self-reported health values in Bhutan as we would have predicted. The self-reported health indicator is used here as a proxy measure and to complement other health indicators (healthy days and disability) and is consequently given only one-tenth of the total weight for health, and only one-third as much weight as any of the other three indicators. For convergent validity, self-rated health status showed a significant positive correlation with health satisfaction level $(\mathrm{r}=.84$, $\mathrm{P}<0.001)$.

The ratings range on a five-point scale from having 'excellent' health to 'poor' health. For a person to be sufficient in self-reported health status, he or she must have a rating of 'excellent' or 'very good'. A large majority $(73.8 \%)$ have met the sufficiency condition in self-reported health.

\section{Healthy days}

This indicator reports the number of 'healthy days' a respondent enjoyed within the last month. Questions regarding the number of physically and mentally unhealthy days per month have been part of the CDC's core Behavioral Risk Factor Surveillance Survey (BRFSS) questionnaire since 1993 (CDC 2000). The total number of unhealthy days which restricted the respondent from performing daily activities is then subtracted from 30 days to get the number of healthy days (Hennessy et al. 1994). This indicator has been validated in a number of studies. However the reference period is only 30 days, whereas the GNH Index is intended to

\footnotetext{
${ }^{48}$ In the case of Bhutan, a multinomial logistic regression was carried out for people having good health status and people with poor health status were compared to it. The dependent variable used is health status (dichotomized) and independent variables used were age, gender, marital status, education level, literacy level, occupational status, region, residential district, household size, electricity and some health components such as distance to health care centers. The number of healthy days and long-term disability were also controlled for. After controlling for the all independent variables, females have a higher risk of reporting lower self-reported health status $(\mathrm{RRR}=1.83, \mathrm{P}<0.000)$ as compared to males. People who are farther from the health care centres $(R R R=1, P<0.05)$, have lower health days $(\mathrm{RRR}=.94, \mathrm{P}<0.000)$ and those who are disabled $(\mathrm{RRR}=.13, \mathrm{P}<0.000)$ have a higher probability of a lower self-reported health status. Similarly, the older population seems to have a lower self-reported health status and some districts seem to have significantly lower ratings of self-reported health status when compared to Thimphu.
} 
capture health status across a longer period of time. The GNH survey does not provide a follow-up question to ascertain whether the past month was average or exceptional in terms of health performance. For that reason, we supplemented healthy days in the past month with the more general self-reported health variable.

The mean number of healthy days for Bhutan is 26 days $(\mathrm{SD}=7.7)$ and the median is 30 days. There is no disagreement that health is an important component of wellbeing, so one possible threshold would be the highest possible value of 30 days. This would identify $34 \%$ of people as being deprived. However health achievements vary, occasional illness is normal, and healthy days are also associated with age (one-way ANOVA, $\mathrm{P}<0.000) .{ }^{49}$ As expected, the elderly enjoy fewer healthy days. So, to allow for normal illness and for elderly respondents, the threshold has been set at 26 days and $76.2 \%$ meet the sufficiency threshold.

\section{Long-term disability}

A person might be characterised as having a long-term disability if he or she has an activity limitation, uses assistance or perceives him- or herself as having a disability, including a mental disability (Wangdi 2009). If this concept is followed, one method used to measure long-term disability would be to examine an individual's ability to perform functional activities of daily living without any restriction (U.S. Department of Health and Human Services 2000). This is the approach followed here. Note that this question focuses on a pragmatic concept, which recognises that disability can occur from birth or be caused by accidents or poor health. Whatever its cause, often the capability of disabled people to function normally is limited by social and environmental barriers. Alternatively, if communities and governments provide sufficient opportunities and support, disabled persons can also enjoy wellbeing.

Participants were asked whether they had any longstanding illness that had lasted over six months. If the answer was 'yes', they were then asked, using a five-point scale, whether the disability restricted their daily activities. The scale ranged from 'never' to 'all the time'. A person was identified as

\footnotetext{
${ }^{49}$ A linear regression model was developed based on healthy days as the dependent variable and demographic features such as gender, age, marital status, educational level, literacy level, occupational status, household size, region and district as independent variables. Additionally, the disability variable and distance from the nearest health care centre were also used as independent variables. Only the coefficients of gender, age, disability and distance from the nearest health care centre were significant. Males enjoyed more healthy days than females, and younger people had more healthy days than the elderly. As expected, disability and distance from a health centre affected healthy days.
} 
having achieved sufficiency if they reported either no long-term disability or if they had reported that it did not limit their ability to perform daily activities. Thirteen per cent of the respondents reported some long-term disability, and about $20 \%$ amongst these disabled were restricted all the time from performing daily activities, and $29 \%$ were restricted often.

The threshold is set such that those individuals who are disabled but are 'rarely' or 'never' restricted from doing their daily chores are classified as sufficient. Conversely, individuals with a disability whose daily activities are restricted 'sometimes' are classified as deprived. With this threshold, about $89.5 \%$ achieve sufficiency.

\section{Mental bealth}

This indicator uses a version of the General Health Questionnaire (specifically GHQ-12) developed by Goldberg. It consists of 12 questions that provide a possible indication of depression and anxiety, as well as confidence and concentration levels. It is calculated and interpreted using the Likert scale with lowest score at 0 and highest possible score at 36. Each item has a four-point scale, but there are two types of scales depending on the structure of statements. The possible responses for some questions range from 'not at all' to 'much more than usual' and some from 'more than usual' to 'much less than usual'. The respondents who reported 'don't know' are categorised under a neutral answer such as 'same as usual'. Note that the percentage of 'don't knows' is less than $0.31 \%$ of respondents for any given question, so there are only very minor changes in the final results.

The General Health Questionnaire has been used extensively since the 1970s in different settings and different cultures, and it is a well-known instrument for measuring minor psychological distress. ${ }^{50}$ The questionnaire was originally developed as a 60 -item instrument, but a range of shortened versions are available including the GHQ-30, the GHQ-28, the GHQ-20, and the GHQ-12. The scale asks whether the respondent has experienced a particular symptom or behaviour in the past four weeks. Once again, a longer recall period would be desirable but was not available. For Bhutan the GHQ-12 was used.

Employing the recommended method of scoring (ranging from 0 to 36), the mean GHQ score was $9.8(\mathrm{SD}=5.82)$. A lower score between the ranges of 0 to 15 indicates normal mental wellbeing, a score between 16 and 20 indicates some mental distress and a high score of 21 to 36

\footnotetext{
${ }^{50}$ See for example, Goldberg and Blackwell (1970).
} 
indicates severe mental distress. A high reliability was observed with Cronbach's alpha coefficient of 0.87 . Convergent validity indicated a significant negative correlation between the GHQ-12 and life satisfaction score as expected $(\mathrm{r}=-0.44, \mathrm{P}<0.001)$. The principal component analysis with oblique rotation showed that the GHQ-12 was a measure of psychological morbidity with two factors and factor one alone explains $62 \%$ of the variance. The threshold was set at normal wellbeing (15) and $85.8 \%$ achieve sufficiency.

\section{Education}

GNH highlights the importance of a holistic educational approach that ensures Bhutanese citizens gain a deep foundation in traditional knowledge, common values and skills. In addition to studying reading, writing, maths, science and technology, students are also encouraged to engage in creative learning and expression. A holistic education extends beyond a conventional formal education framework to reflect and respond more directly to the task of creating good human beings. It is important for Bhutan that an education indicator includes the cultivation and transmission of values (Ura 2009).

To understand education in Bhutan, it is necessary to note that a Westernstyle curriculum began in Bhutan in the 1950s. Before that, monastic education was the only formal education available. ${ }^{51}$ While the Western form of education established an instrumental approach that remains a key vehicle for productivity, employment and higher earnings in Bhutan, it is equally important to recognise and promote the consideration of ethical values as the basis of a good educational practice. The link is also of instrumental interest: studies also show strong associations between the likelihood of criminality and educational attainment (Lupton and Power 2005; Fagan and Davies 2007; Friedman 2010). The approach known as 'values education' has been recently introduced across Bhutan (Ura 2009). This is further emphasised in Bhutan's constitution which states that the country '....shall endeavour to provide education for the purpose of improving and increasing knowledge, values and skills of the entire population with education being directed towards the full development of the human personality.' So, it is important for Bhutan that an education indicator includes the cultivation and transmission of values (Wangyal 2001). Also, although school education occupies some space in the process of imparting knowledge, there are phenomena outside of schools that play equally important roles such as communities and families (Ura and Zangmo 2008).

51 http://www.education.gov.bt/Edn\%20System/Education\%20System.html 
In an attempt to reflect the holistic aspects of education, four indicators literacy, educational qualifications, knowledge and values - were considered.

\section{Literacy}

To reflect the learning that those who do not have primary schooling have achieved, as well as to re-affirm the learning of those who do, we begin by measuring literacy. A person is said to be literate if he or she is able to read and write in any one language, English or Dzongkha or Nepali. Including literacy makes the overall education measure more accurate. For example, in the $2010 \mathrm{GNH}$ survey, $1.9 \%$ of respondents, mostly farmers, had achieved six years of schooling but were not literate, suggesting some quality issues in rural schools. Also, $13.6 \%$ of Bhutanese are literate although they have not had six years of schooling - either because they are self-taught, or because they had some schooling but did not complete primary school, or because they attended a non-formal education programme (a number of these have been offered free by the government). Most Bhutanese who have achieved six years of schooling are also literate, and this measure therefore recognises their educational achievements. In literacy, $48.6 \%$ have attained sufficiency. Schooling on a universally accessible basis grew from the 1970s onwards. The backlog of older generations who did not go to school is revealed by the low literacy rate.

\section{Educational qualification}

The education system in Bhutan has two major components: formal education and non-secular institutions such as monastic schools. Nonformal education (NFE) was also started in the 1980s to provide functional literacy to the target groups such as the elderly. This educational indicator includes formal schooling, education imparted by monastic schools and NFE.

Where should the threshold be set? On the one hand, the Constitution of Bhutan (2008) states that, 'The State shall provide free education to all children of school going age up to tenth standard ...' This might suggest that from 2008, the sufficiency cutoff for education should be ten years. However, note that ten years of education in Bhutan is not compulsory so that would seem too stringent. The Tenth Plan of Bhutan states that 'The national goal is to achieve near $100 \%$ enrolment at primary education.' Primary schooling lasts six years for Bhutanese, hence six years is the minimum legal requirement. One might think that an even lower threshold might apply for older persons who did not have the opportunity to attend 
school as children, when the national goal was lower. ${ }^{52}$ However, if learning has intrinsic value, then it can add value for someone of every age. Further, Bhutan has adult educational programmes and programmes for disabled people. Also, the society in general stresses the value of equality among persons, so having different thresholds for youth would not enable the GNH to depict inequalities across generations. For these reasons, the threshold for education was set such that persons have insufficient education if they have not completed six years of schooling from any source, including government, non-formal, or monastic schools. With this threshold, only $37.3 \%$ have attained six years of schooling, again due to the fact that schooling and non-formal education began relatively recently in Bhutan.

\section{Knowledge}

The knowledge questions attempt to capture learning which could have occurred either inside or outside formal institutions. Family and community play equally important roles in creating a learning environment, and the media contributes as well. To validate such knowledge, five knowledge variables were chosen based on their importance and their applicability to all sections of society in the country. They are: knowledge of local legends and folk stories, knowledge of local festivals (tshechus), knowledge of traditional songs, knowledge of HIVAIDS transmission, and knowledge of the Constitution. Bhutan 2020 (Royal Government of Bhutan 1999) states the importance of educating people in the rich folklore, myths, and legends that transmit values and act as foundation for inculcating '.....an awareness and appreciation of the continuing and contemporary relevance of our culture and heritage to development of the individual, their families, their communities and the nation.' Further, knowledge of local legends and folk stories has a spiritual significance, as well as acting as a medium of entertainment and celebration (Kinga 2001).

The recent Constitution (2008) which transformed Bhutan from a monarchy to a constitutional monarchy provides the legal framework for the government, designates the powers and duties of the branches of government or governmental agencies, and establishes the relationship between the people and the government. Moreover, it enunciates the basic rights and obligations of citizens and encourages their political participation - which is vital for the success of democracy. For these

\footnotetext{
${ }^{52}$ For example in the $5^{\text {th }}$ five-year plan (1981-1986) primary school lasted five years only, whereas the $2^{\text {nd }}$ five-year plan (1966-71) did not emphasise providing universal education "at this juncture."
} 
reasons and others, it is crucial for people to have at least some knowledge of the Constitution. Knowledge currently appears to be deficient, with only $1.6 \%$ claiming 'very good' knowledge.

Since the human immunodeficiency virus (HIV) was first detected in Bhutan in 1993, the prevalence of HIV infection has increased in urban areas. Rates in much of the rest of Bhutan are much lower; nevertheless, these rates pose a considerable public health threat unless people have a good understanding of HIV/AIDS or the ability and inclination to behave in a low-risk fashion, or both. Only $30 \%$ of people claim to have a good understanding of how HIV/AIDS is transmitted.

The responses for each knowledge question follow a five-point scale which ranges from 'very good knowledge' to 'very poor knowledge'. Responses are aggregated to create a maximum score of 25 which indicates 'very good' knowledge in all areas, while the minimum score of 5 indicates 'very poor' knowledge. The average knowledge score was $12.7(\mathrm{SD}=3.7)$ and the median score was 12. A significant positive correlation was demonstrated between education level and knowledge $(r=.5)$.

The threshold is set to 19 which implies that Bhutanese should have an average of 'good' knowledge across the five variables. This additive indicator allows for compensation across different kinds of knowledge. When the threshold is applied, only 7.5\% have sufficiency in knowledge. Sufficiency in knowledge is low compared to other indices; only 3\% rated 'good' or 'very good' in all five knowledge indicators. It suggests a divergence between rising literacy and declining knowledge about their respective locality.

\section{Values}

Values are fundamental to human beings as they shape people's character and the choices they make in their lives. The development of children's value systems begins at home and is continued in schools and shaped also by organizations and communities. Whether the values are channelled from an educational institution or from family or communities, traditional values have always had a huge role in shaping behavioural changes in the Bhutanese people. Based on Buddhist culture, they have been highly valued by citizens for centuries (Wangyal 2001). Bhutan 2020 (1999) states the desirability of cultivating '.. universal values that develop the capacity of our young people to distinguish right from wrong, good from evil, and to lead lives that are guided by moral and ethical choices.' The self-critical yet articulate cultivation of social and moral values is becoming more intentional as Bhutan modernises, because positive values enable people to 
manage the complexities that arise from the fast-changing environment. A concern for cultivating values has been also outlined in the Bhutan Development Report (Royal Government of Bhutan 2000) which states, 'Happiness in the future will also depend upon mitigating the foreseeable conflict between traditional cultural values and the modern lifestyles that inevitably follow in the wake of development.' As the market takes its place in a society, there is a real risk of generational clashes and alienation.

The 2010 GNH indicator of values used may be revised in future GNH surveys but provides some preliminary insight into these issues. Respondents were asked whether they considered five destructive actions to be justifiable: killing, stealing, lying, creating disharmony in relationships and sexual misconduct. In a society influenced by good values, e.g. by Buddhism, individuals are expected to tame themselves with respect to these five destructive actions. Moral consequences of virtues and nonvirtues are typically revealed through speech, body and mind and in the case of disinformation, the agency of speech is emphasised. The variables have a three-point response scale ranging from 'always justifiable' to 'never justifiable' along with an option of 'don't know'.53 The values have been combined into a composite indicator in a particular manner. For killing, stealing and sexual misconduct, a value of 1 is assigned if the person reports 'never justifiable' while for creating disharmony and lying, responses either 'never justifiable' or 'sometimes justifiable' are assigned 1. The composite indicator takes the values 1 to 5 .

The threshold is set at four which implies that a person can consider at least one of the values to be justifiable. This allows for diversity in interpretations and ethical frameworks, as well as response bias. For example, with respect to the question on killing, there is a difference between killing a human being and killing an ant; there is also a difference between killing intentionally and killing inadvertently. The statement does not clarify the object nor the intention associated with the killing. Similarly the question does not specify whether it is a 'white lie' or a serious deception, whether stealing an egg or a herd of yak. This means that people's answers will depend in part upon their interpretation of the question, and these may vary. Second, it allows a diversity of ethical frameworks. Opinions as to whether certain killings may be justified will vary among followers of different faiths and philosophies. Third, the responses to the questions again might reflect some self-consciousness on the part of the respondent, whose responses might be biased by what they

\footnotetext{
53 An examination of the underlying factor structure resulted in a single factor with loadings above 0.5. Internal consistency was sufficient (Cronbach's alpha of .65) to allow computation of an indicator.
} 
believe the expected or 'socially acceptable' answer to be. The response structure enables people to say that some negative actions are 'sometimes justified' - such as lying and creating disharmony in human relations. But it cannot correct a positive bias in the underlying data. Even with a threshold of 5 (maximum score), about $85.2 \%$ are classified as sufficient in values. However, to allow diversity, the threshold is set at four and $97.1 \%$ achieve sufficiency in value. The $2010 \mathrm{GNH}$ indicator of values used will be improved in future GNH surveys but the present finding provides some preliminary insight into these issues.

\section{Culture}

The distinctive culture of Bhutan facilitates sovereignty of the country and provides identity to the people. Hence the preservation and promotion of culture has been accorded a high priority both by the government and the people. The importance is evident in Section 1, Article 4 (Culture) of the Constitution of Bhutan which states that the country, '... shall endeavour to preserve, protect and promote the cultural heritage of the country....' Further, culture is not only viewed as a resource for establishing identity but also for 'cushioning Bhutan from some of the negative impacts of modernization...' (Bbutan 2020, Royal Government of Bhutan 1999).

The diversity of the culture is manifested in forms of language, traditional arts and crafts, festivals, events, ceremonies, drama, music, dress and etiquette and more importantly the spiritual values that people share. It is visible in the daily lives of people and therefore plays a dominant role in moulding the Bhutanese character and way of living.

While accepting that Bhutan has a diverse and unique set of cultures to be protected, it must be noted that culture is also dynamic concept, constantly evolving and continuously challenged by external forces and by internal cultural and social change. Therefore, sustaining these cultural aspects requires continuous promotion and progress towards developing adequate resilience (Chophel 2010).

To assess the strength of various aspects of culture, four indicators have been considered: language, artisan skills, cultural participation and Driglam Namzha (the Way of Harmony).

\section{Language}

Bhutan is a country with a diversity of languages, and they are an important component of culture as they provide each socio-cultural community with a sense of identity, history and culture. The national 
language Dzongkha is a symbol of national identity in Bhutan and so, to assess literacy, an indicator that includes literacy in Dzongkha has already been incorporated into the education domain. The language indicator here pertains to the ability to understand and speak one's mother tongue. Bhutan is a multilingual country with about 19 different languages. Language is not considered just as a communication tool but also a source of identity and social integration and cultural development.

The language indicator is measured by a self-reported fluency level in one's mother tongue on a four-point scale. It should be clarified that a mother tongue is defined as a natal tongue which is a dialect. There are over a dozen dialects. Only in Western parts of the country does the mother tongue coincide with the national language, Dzongkha. The ratings vary from 'very well' to 'not at all'.

In some countries the native languages are being forgotten by people, especially the younger generation, in favour of the national or international languages. Fortunately in Bhutan the mother tongue still seems vibrant as about $95.2 \%$ of the respondents speak their mother tongue 'very well', and amongst the teenagers, $86 \%$ speak it 'very well'. However, with external influences such as the media, television and the internet, people are focusing more on global languages rather than own their own. Languages such as English are increasingly gaining popularity in families of urban areas. Of course, it is understood that the influences that fluency in English brings are not necessarily negative. On the contrary, they often prove to be enriching. But, because of the accelerated emphasis on the English language, there is an increasing threat to the native languages of Bhutan. So, it has become important for Bhutanese to sustain proficiency in their mother tongue and to promote it among the younger generations. Since, currently almost everyone seems to be fluent in their mother tongue, a high threshold is necessary to maintain standards. And for this reason, the threshold is set to 'very well'. With this threshold, at present an impressive $95.2 \%$ of respondents are classified as sufficient.

\section{Artisan skills}

Unlike many countries, traditional arts and crafts in Bhutan are not remnants of a bygone age but a vibrant aspect of culture which has been practiced for generations. They are also reflected vibrantly in the everyday lives of artisans. Their artistic expression and beautiful crafts represent ancient knowledge and also serve secular and spiritual functions (Bhutan 2020, Royal Government of Bhutan 1999). 
There are thirteen artisan skills, collectively known as Zorig Chusum. These skills draw upon a long tradition deeply imbedded with spiritual significance. Although these skills continue to retain their relevance in bringing contentment and happiness to the minds and lives of people, it will be increasingly challenging to preserve their spiritual significance due to modernisation (Wangdi 2009). Yet the Zorig Chusum skills are very much part of Bhutanese culture and represent a source of cultural capital. In order to promote and preserve culture, it is vital to include an indicator which assesses people's interest in and knowledge of Zorig Cbusum and reports on the number of skills possessed by a respondent. These skills and vocations are the basis of historical material culture of Bhutan when it was trading far less. The 13 arts and crafts include 1) weaving (Thagzo) 2) embroidery (Tshemzo) 3) painting (Lhazo) 4) carpentry (Shingzo) 5) carving (Parzo) 6) sculpture (Jinzo) 7) casting (Lugzo) 8) blacksmithing (Gar:o) 9) bamboo works (Tszharzo) 10) goldsmithing and silversmithing (Serzo and Nguelzo) 11) masonry (Dozo) 12) leather works (Kozo) and 13) papermaking $(D e z o)$. For the indicator, people were asked if they possessed any of the above 13 arts and crafts skills. The mean was 1.01 with a SD of 1.15 .

Both the mean and median number of skills is 1 ( $S D=1$.) Sixty-two per cent seem to have at least one skill while only $22 \%$ have at least two skills. A sufficiency threshold has been set at one, which implies that a person must possess at least one skill to be identified as sufficient. About $62 \%$ of the respondents are categorised as having achieved sufficiency. The dominant or commonly shared skills today are masonry, carpentry and textile weaving.

The Zorig Chusum indicator does not only aim to encourage people to learn multiple skills; it also enables an assessment of the type of skills possessed and the skills that are less frequently practiced and thus deteriorating. For instance, the most common skill amongst people was 'weaving' with $32 \%$ possessing the skill and 'goldsmithing and silversmithing' was the least practiced, with only $0.32 \%$ of the respondents skilled in them. The rest range from $1 \%$ to $16 \%$. The result shows that most skills are practiced by a small number of people.

\section{Socio-cultural participation}

Cultural festivals and events, an expression of Bhutan's ancient culture, continue to have a special significance in the daily lives of the people. The community festivals and social gatherings not only contribute to cultural vitality but also bring together people to share joy and happiness. Such cultural events acts as a medium to remind, retain, disseminate and transmit cultural heritage. There are studies which also confirm that 
participation in cultural and social activities has a positive effect on health, in addition to promoting local pride and a sense of belonging. ${ }^{54}$ Participation also helps build social capital based on strong social networks and relationships and thereby builds social connectedness in the community. To measure the level of socio-cultural participation in the country, the respondents were asked the number days they spent in the past year attending various socio-cultural events.

There is growing evidence that cultural participation builds and enhances cohesiveness and connections within a community as well across communities. As Robert Putnam (2000) describes, civic engagement such as meetings or voting might be important but not inspiring or fun. On the other hand people often participate in cultural events out of pure enjoyment. As a result the social capital created over such participation is more stable and helps to develop strong bonds across differences in communities. In Bhutan, the cultural participation also has a major role in the spiritual life as most cultural events have a deep spiritual and historical significance.

In order to assess people's participation in socio-cultural activities the average number of days within the past 12 months is recorded from each respondent. The days are grouped on five-point scale ranging from 'none', and ' 1 to 5 days' to ' +20 days'. The median is 1 to 5 days and mean is 6 to 12 days. About 15\% spent more than 13 days attending socio-cultural events in the past year and 1\% reported 'don't know' (these respondents were dropped).

At present there is no cultural standard as to how many days should be sufficient for cultural participation. So it is vital to take into account some normative considerations. As the days of participation depends on the number of cultural events taking place, perhaps using the total number of events in a year might be one way of setting the threshold. But actually the total possible days will vary across communities and regions. Based on GNH norms, members of community must be active participants and must make a continual effort to participate in such events. Setting a threshold as per the median 1 to 5 days would classify $95 \%$ of Bhutanese as sufficient because only $6 \%$ do not attend at all. Yet attending just one cultural gathering in a year (1 day) would not provide a strong basis for creating shared ownership of the community. In Bhutan, the number of events in communities exceeds five days annually in most regions. So, the

${ }^{54}$ Chouguley, Naylor and Rosemberg-Montes (2011). 
threshold was set at 6 to 12 days per year. ${ }^{55}$ It identifies $33.2 \%$ to have achieved sufficiency.

\section{Driglam Namzha}

Driglam Namzha (the Way of Harmony) is expected behaviour (of consuming, clothing, moving) especially in formal occasions and in formal spaces. It arose fundamentally from the conventions of communal living and working in fortress-monasteries. Certain elements of Driglam Nam₹ba are commonly practiced amongst Bhutanese when they interact with each other in formal spaces. A minimal part of it is also taught for a few days in educational institutions. Respondents were asked to rate its importance on a three-point scale of being very important to not important. In addition, respondents were also asked if there were any perceived changes in the practice of this particular form of etiquette over the years.

For Driglam Namzha, two indicators were developed: perceived importance of Driglam Namzha and the perceived change in practice and observance during the last few years. The questions run on a three-point scale: perceived importance ranges from 'not important' to 'very important' and perceived change from 'getting weaker' to 'getting stronger'. Both have values of 'don't know' which have been classified as insufficient since it is considered vital to have knowledge about etiquette. In order to create conditions for social harmony it is essential to understand and acknowledge the importance of Driglam Namzha. Equally important are the trends of how it is being practiced by society at large, as individual perceptions are affected to a large extent by the appreciation and expressions of such practices in society.

The thresholds have been set at 'important' for perceived importance and at 'getting stronger' for perceived change. Both indicators need to be fulfilled for an individual to be identified as sufficient in Driglam Namzha. After applying the thresholds, $59.7 \%$ of people enjoy sufficiency.

\section{Time use}

The balance between paid work, unpaid work and leisure are important for one's wellbeing. Similarly, a flexible working life is vital for the wellbeing of individual workers and their families and communities. The value of time-use information lies in the fact that time is the ultimate resource and unlike other resources, time is shared equally by everyone (Fleming and Spellerberg 1999). Further, time-use data is an important resource which

\footnotetext{
55 It may be that in future surveys the response categories might be revised.
} 
brings into view voluntary work in communities and domestic work at home besides providing an overview of time spent in both the production and consumption of goods and services. So, in a sense, time use is all encompassing by its nature as it incorporates the activities carried out in the other eight domains of GNH.

Time-use data provides a comprehensive view of how individuals use their time within a 24-hour period, and this can be used to provide information on his or her work life balance (Galay 2009). Many studies have confirmed the importance of work-life balance on health. For instance, time pressures often act as stressors and have a critical impact on health. Proper usage of time has also been recognised as the key ingredient in building relationships (Bohen and Viveros-Long 1981; Staines and Pleck 1983; Beach 1987). The literature also suggests that there is a correlation between working long hours and fatigue and poor mental health.

Since the 1970s, there has been a growing awareness of how unpaid work both at home and in communities is obscured in national accounts and so efforts have been made to include these activities, which are equally fundamental to wellbeing. Some statistical offices such as the OECD are producing extended national accounts which include satellite accounts of domestic production based on time-use data (OECD 1995; OECD 2009). Similarly, the Human Development Report 1995 focused on women's status worldwide and demonstrated that if unpaid and paid work are considered together then women are found to do a larger share of work in both developing and developed countries.

When it comes to measurement, a variety of methodologies are available to examine the time use of individuals. The most accurate method of collecting data on time use might be to observe the respondents carrying out the activities directly; however, such a strategy is extremely expensive and often intrusive (Robinson and Godbey 1997). An alternative is to provide a pre-coded list of activities for which respondents provide frequency and duration of participation in the respective activities (Yoshida and Nakano 2007). This method requires the activities to be narrowly defined and has been shown to have poor reliability for persons with disabilities. Lastly, there is the time diary and time budget which is self-administered or interviewed with fixed intervals to be filled in during randomly designated diary days (Singleton and Harvey 1995; Harvey et al. 1996; Robinson and Geoffrey 1997; Sorokin and Berger 1939; Szalai 1972; Eurostat 2009; United States Bureau of Labor Statistics 2010).

In the GNH survey, a simple time diary was administered. Information on how people use their time was collected by asking respondents to recall 
their activities during the previous day. Survey respondents reported activities that they did from the time they woke up until the time they slept on the previous day of the interview. For each activity the respondents were asked how long the activity lasted. The activities were then later regrouped into 60 different categories spent on different kinds of activities such as work, leisure, sleep, personal care and so on.

Time-use data can yield a range of important information that provide insight into lifestyles and occupations of the people. It can also reveal the gap between GDP and non-GDP activities, which reflects the gap between market and household economy sectors. Such data are helpful in accounting for a more comprehensive output of goods and services that the system of national accounts omits (Ironmonger 1999). Time-use data on 24 hours in the lives of the Bhutanese people can be broken down into various useful sub-categories. The distribution involves the following disaggregation: 20 districts, 7 income slabs, 11 age groups, 60 activities, and gender (Ura 2012). However, the GNH Index incorporates only two broad aggregated indicators of time use: work hours and sleep. The definition of work hours in GNH is not completely congruent with definitions used elsewhere and shows unusually long work duration in Bhutan. Some activities not usually defined as work elsewhere are included as part of work.

\section{Working hours}

There are many ways of defining long hours. For instance, daily, weekly or annual hours spent in an individual's main occupation and sometimes commuting time to a workplace are considered when working hours are calculated. Working hours in the GNH definition includes even unpaid work such as child care, woola (labour contribution to community works), voluntary work and informal help. In this indicator, all the following categories are classified as work: crop farming and kitchen gardening (agriculture); business, trade and services; the care of children and sick members of household; construction and repairs; craft-related activities; forestry and horticultural activities; household maintenance; livestockrelated activities; the processing of food and drinks; and quarrying work.

In terms of thresholds, many studies seem to focus on the classification set by the International Labour Organisation (ILO 1919), which sets working limits at 48 hours per week (eight hours per day) for manufacturing. Working beyond that limit was considered unhealthy (Spurgeon 2003). Most researchers seem to identify a maximum threshold of 48 hours per week or 8 hours per day. Dex, Clark and Taylor (1995) discussed that long hours may be considered differently for men (over 60 hours per week) and 
women (over 40 hours per week). Many such surveys also highlight the stress experienced when working long hours. It is possible that the relationship between hours of work and ill health is created by stress.

Eight hours is also the legal limit, applied to formal sector, set by the Ministry of Labour and Human Resources of Bhutan for a standard work day. Working time in service sectors or other non-formal sectors has not been defined. The survey results show average working hours to be eight $(\mathrm{SD}=3)$ while the median is 8.5 hours. A few respondents worked as many as 16 to 20 hours on previous days (mostly in rural areas). In most cases this was time spent either volunteering at funeral rites or preparing for seasonal sociocultural gatherings.

By age, Bhutanese people work the longest hours in midlife (46 to 50 years old). At that age, they work 518 minutes on average a day. Sleep duration contracts and is the shortest at age 44 to 45 and improves only a little until age 55. After 55, sleep increases.

Rural women work for 8 hours and 43 minutes (524 minutes) a day, the longest of all. Rural men work 7 hours and 46 minutes a day, almost an hour less than rural women. Urban men work the fewest hours $(7$ hours 3 minutes or 424 minutes). In almost all dzongkhags, people in towns work less than their counterparts in villages, paralleling occupational differences between farmers and non-farmers. Towns and cities therefore emerge as places with a lower work burden.

Data indicate that the lower a person's educational level is, the more time the person must spend on work in Bhutan. In other words, as education increases, the amount of time spent on work decreases. The unemployed also work in Bhutan and the bulk of work they do is household maintenance work. They are not idle; they work six hours a day on household maintenance, business, crafts production, and care giving and various other activities defined as work. This is one of the significant paradoxes that can mislead policymakers to devalue the status and contribution of the unemployed. The unemployed are contributing substantially to the household economy.

It is quite clear that, regionally, the people in eastern districts of Tashiyangtse, Mongar, Tashigang and Samdrup Jongkhar work much longer than those in Thimphu, Punakha and Paro. Leisure hours, broadly defined, have grown relatively longer in Western districts. Paro and Tashiyangtse are two extremes on a continuum. On an average, people in Tashiyangtse work 64 minutes longer per day than the people in Paro, a 
dzongkhag where road connectivity is high and wildlife pose almost no risk to agriculture.

The district variations in the length of working hours are also a broad reflection of cash income levels. Lower household cash income status coincides with longer working hours and this relationship is more pronounced in Tashiyangtse, Mongar, Tashigang and Samdrup Jongkhar. Nationally however the positive correlations between household income and duration of hours worked is quiet weak $(r=0.044) .{ }^{56}$ The long working hours and lower household income of a farmer in Eastern Bhutan has serious implications for many dimensions of wellbeing. Under five mortality, for example, is much higher in Eastern districts than the rest of the country (87 per 1000 for Eastern districts, vs 61 for Western and Central districts), and surprisingly, higher nationally among male than female infants (79 versus 58 per 1,000) (NSB, 2011, p. 28).

Cooking is not only predominantly women's work in both urban and rural areas, the average time spent per day on cooking is longer for women. On average, a man who cooks spent 71 minutes in kitchen while women spent 117 minutes cooking. One would expect urban people to take less time cooking because of the presence of time-saving devices, but there is no significant difference in the time spent in cooking between either places. Eating takes 87 minutes a day on average. If you have the fortune of living 70 years, 4.2 years will be spent eating and drinking.

Among those who own TV sets - and about $50 \%$ of households did in 2010 - rural Bhutanese watch it for 41 minutes. Urban Bhutanese spent an extravagant amount of time watching TV - watching it for an imposing 174 minutes, on average 25 minutes longer on TV watching than rural TV owners, perhaps while also doing some other things. Other international surveys noted that when engaging with media, attention is not exclusive to it. Watching TV might be combined with cooking meals. The $2010 \mathrm{GNH}$ data cannot capture simultaneous tasks, forcing every activity to claim $100 \%$ of human attention and assuming the sequential performance of every task. However, simultaneity of work is also completely omitted in SNA market activities estimated in any GDP (Blackden and Wodon 2006, p. 58).

\footnotetext{
56 It is intriguing that there is no strong negative correlation between household income and duration of work $(\mathrm{r}=-0.044)$ i.e. higher income is not correlated with lower work hours. The expected inverse relationship between lower household income and lower amount of time spent on non-work was also not found $(\mathrm{r}=0.07)$. Usually, social, cultural and leisure activities would be squeezed out by working for a living at the lower income level.
} 
Eighty-one per cent of Bhutanese are Buddhists, 18\% are Hindus, and $1.2 \%$ are Christians, according to GNH 2010 survey. As expected, religious activity ${ }^{57}$ for most Buddhist Bhutanese consists of two activities: praying and making offerings of water and butter lamps. But in terms of time allocation, praying takes far more than making offerings, which requires only 15 minutes on average with virtually no gender difference in time expenditure and is done by fewer Bhutanese (34\%). Praying is not a universal activity. On a typical day, that is not a holy holiday, around $40 \%$ of Bhutanese engage daily in prayers. Prayer recitation averages an impressive 228 minutes $(S D=109)$ a day ( 3 hours 48 minutes) for the clergy (lay priests, nuns and monks). But the average plunges to 79 minutes for lay people $(\mathrm{SD})=109)$ if we net out the specialization in this field by clergy. But these 79 minutes are under pressure due to an increase in time spent watching TV.

Those who gave care to children, the elderly, the sick and the disabled formed $25 \%$ of the population on the day of the survey. They spent 81 minutes per day per person on it. While all other broad occupational groups spend an average of 16 minutes on compassionate activities, housewives devote the most, spending 51 minutes a day. Paid care and public sector care, which have official health cost implications, will increase if this time allocation by housewives decreases for any reason.

Social contacts must be still strong in Bhutan. About 34\% of the Bhutanese socialize every day, socializing with members of family for an average of 63 minutes and another 89 minutes with friends and neighbours and relatives.

Travelling to and from work averages one hour for Bhutanese in general, but it is significantly higher for two kinds of employees. The National Work Force spends an average of 1.28 hours per day travelling to work, closely followed by civil servants who spend 1.16 hours.

Being overworked compromises time for leisure, family time and other social activities that are equally beneficial for wellbeing. Since a main objective of the indicator is to assess people who are overworked, those who work for more than eight hours are identified as time deprived. Forty-

\footnotetext{
${ }^{57}$ The activities which constitute religious activities are sub-divided into chanting prayers or mantras or counting beads; offering water or food or incense or a butter lamp; conducting or organising rituals; meditating; prostrating; circumambulating choetens and lhakhangs; hoisting prayer flags; attending of religious teachings; and local pilgrimage. Although separate mutually exclusive time aggregates for these activities should be available, in practice respondents reported on the first two sub-activities. The rest were negligible percentages of the total time.
} 
five point four per cent achieve sufficiency when this threshold is applied. Those who do not achieve this sufficiency are mainly women irrespective of whether they live in towns or villages, and more generally the people in the Eastern districts. People in Eastern Bhutan have longer work days compared to the rest of the country.

\section{Sleeping bours}

Sleep is clearly beneficial for a person's health and impacts nearly every area of daily life. Sleep also plays a vital role in promoting longevity and emotional wellbeing. But many people do not realise how much sleep is required. Sleep requirements can vary substantially. In general most healthy adults need an average of seven to eight hours of sleep for proper functioning (Kleitman 1963; Doran, van Dongen and Dinges 2001; Smith, Robinson and Segal 2011). It is also important to understand the factors affecting one's sleep time. ${ }^{58}$ Individuals in some occupations, such as nuns and monks, prefer and find it much healthier to devote more time to meditation and other spiritual practices than sleeping. Indeed, the survey confirms that they sleep comparatively less. Although such adjustments are important, it is not yet possible to determine specific sleeping requirements for specific sections of societies. Therefore, we use a uniform cutoff.

Eight hours of sleeping time is considered the amount necessary for a well-functioning body for everyone. Both the mean and median fall around eight hours for the respondents. With this threshold, about $66.7 \%$ achieve sufficiency.

Twenty-four hours can be broadly divided into segments devoted to work, non-work and sleep. In Bhutan, on average, within a typical 24 hours, people spend $35.3 \%$ of it on sleep, $32.3 \%$ on work and $32.1 \%$ on nonwork; but this broad tripartite distribution masks variations in the distributions among different groups. Non-work consists of personal care, socio-cultural activities, religious activities, leisure including watching TV. The general picture of time use in Bhutan depends on many factors.

\footnotetext{
58 A multi-linear regression $(\mathrm{N}=7086)$ is carried between sleeping time and demographic factors such as gender, age, occupational status, educational qualification, and region, represented by the number of healthy days. Age and some occupations and regions were found to be significant. The elderly sleep less than the younger people (coef $=-.284$, $\mathrm{P}<0.05)$. Similarly, occupational groups like farmers, military $(\mathrm{RBP} / \mathrm{RBG} / \mathrm{RBA})$ and monks and nuns sleep less than civil servants. Note that monks and nun might be intentionally sleeping fewer hours since time is devoted to meditation and prayer recitations.
} 
On average, Bhutanese sleep 509 minutes, or 8 hours and 29 minutes $(\mathrm{SD}=97)$. Amazingly, rural women who work longer hours do not do so at the expense of sleep. They sleep 522 minutes a day. They also sleep just a little longer than rural men (a mere 12 minutes). This is possible because they compromise slightly on the non-work component of their day. Twelve minutes seems a fleeting and insignificant difference, but over a year it adds up to 4,320 minutes or 72 hours of sleep. Even urban women sleep a little longer duration, by 17 minutes, than urban men.

'The principle thief of time is sleep' wrote (Robinson et al. Cited in Szalai A et al., p. 132). Some keep the thief away a little longer. The distinction of wakefulness belongs to clergy, the national workforce and the armed forces. Soldiers take turns to be sentries at night in addition to their regular day duties, cutting into their sleep time. The case of less sleeping time among the clergy (monks and nuns and lay priest) may be special. The senior members may be praying or meditating by their own choice while younger ones might be rudely awakened for pre-dawn group prayers.

\section{Good governance}

Many definitions of good governance have been coined in literature, hence the relevant concept is particular to the vision and goals of the country and to the approach of governance being followed. In general, some of the key attributes are participation, rule of law, transparency, accountability, efficiency, effectiveness, responsiveness, a consensus orientation, equity, empowerment and inclusiveness (World Bank 1992; Canadian International Development Agency 1996; Kaufmann 2005). These principles also reflect the values that need to be implemented in order to justify the governance framework. In Bhutan they have been at the heart of any public policy in the country. Further, it is reaffirmed by Article 20 (The Executive) of the Constitution of Bhutan which states, "The Government shall protect and strengthen the sovereignty of the Kingdom, provide good governance, and ensure peace, security, wellbeing and happiness of the people.'

In an effort to reflect much of the principles mentioned above, four measures were developed to signify effective and efficient governance. These include fundamental rights, trust in institutions, performance of the governmental institutions and political participation. It must be noted that these indicators may be adjusted in future surveys. The governance indicators are quite innovative in combining political activities with access to government services. These are understood as part of governance and a part of the public services to be provided by the government. It also includes fundamental rights to vote, freedom of speech, join a political 
party, to be free of discrimination and a perceptual indicator on government performance.

\section{Political participation}

Active political participation and civic engagement are central to creating a vibrant democracy. In addition, studies show that people who participate in political activities enjoy higher wellbeing because of the resulting feelings of freedom and autonomy (Weitz-Shapiro and Winters 2008). The economist Amartya Sen speaks of the freedom to participate as being a key form of development in his book Development as Freedom. He views participation in making decisions that affect one's life and the lives of others as fundamental to human wellbeing, 'Participation can also be seen to have intrinsic value for the quality of life. Indeed, being able to do something through political action-for oneself or for others-is one of the elementary freedoms that people have reason to value' (2002, p. 359).

Further, political participation might increase citizens' knowledge and competence regarding specific issues, and also, perhaps more importantly, about the nature of the political process and even their own rights as citizens.

The measure of political participation was based on two components: the possibility of voting in the next election and the frequency of attendance of zomdue (community meetings). The respondents are asked if they would vote in the next general election and the response categories are simply 'yes' or 'no' or 'don't know'. To calculate the frequency of attendance, the most common meetings are described to the respondent such as block, village and municipal level. These three levels are summed up to provide the total number of meetings attended in the past year.

An individual has to report 'yes' in the voting criteria and has to attend at least one meeting in a year to be classified as sufficient in political participation. About $92 \%$ have expressed an intention to vote in the next general election, $4.7 \%$ declined and $2 \%$ don't know. For voting, the threshold is straight forward because it is agreed by everyone that developing true democratic processes requires the active participation from citizens - minimally, by voting. Moreover, GNH aims for a society where citizens have not only the privileges of rights but also the responsibility for voicing their opinions regarding elected leaders and overall policies. In terms of attendance of meetings the threshold has been set to one time. About $60.2 \%$ attended at least one meeting. Fixing the threshold as such classifies $43.6 \%$ as deprived in political participation. 


\section{Political freedom}

These indicators attempt to assess people's perceptions about the functioning of human rights in the country. Basic fundamental rights are enshrined in the Constitution of Bhutan which has an entire article (Article 7, Fundamental Rights) dedicated to it. They are composed of the basic freedoms and conditions essential for the development of every citizen and also establish the framework for democratic rule. The seven questions related to political freedom ask people if they feel they have freedom of speech and opinion, the right to vote, the right to join the political party of their choice, the right to form tshogpa (association) or to be a member of tshogpa, the right to equal access and the opportunity to join public service, the right to equal pay for work of equal value, and freedom from discrimination based on race, sex etc. All have three possible responses: 'yes', 'no' and 'don't know'.

The thresholds for all rights were set to 'yes'. So, a person has a sufficient condition in the indicator if he or she has all seven rights fulfilled. Of the respondents, $61.7 \%$ were identified as sufficient.

\section{Service delivery}

The central function of the government is to provide services to the people. Some of the basic services, which in Bhutan are usually government-provided, have been included in the indicator in order to evaluate objective access. The indicator comprises four indicators: distance from the nearest health care centre, waste disposal method, access to electricity and water supply and quality. The goal is to evaluate access to such basic services, which in Bhutan are usually provided by the state.

Health services play a vital role throughout the life cycle. Respondents are asked the walking distance to the nearest health care centre. People less than an hour's walk are considered to have sufficient access.

The management of waste can have a significant impact on environment and human health in Bhutan. Respondents were asked about their waste disposal method in order to assess the situation and identify possible areas of improvement. With environmental conservation being placed at the centre of Bhutan's development strategy, it is vital to create effective waste management systems. As in most countries, the handling of waste varies by location. For instance, landfills are prevalent in urban areas where there are facilities for garbage trucks, so $62.3 \%$ of the urban dwellers manage their garbage via municipal garbage pickup. In contrast $64 \%$ of the respondents dwelling in rural areas burn their waste and $31 \%$ use 
composting. Elsewhere, recycling is considered to be one of the best waste management strategies; however in Bhutan there is much to be done in terms of establishing a strong base for recycling. And so the present indicator has no option for 'recycling' but perhaps in future this option must be included once waste management allows this option.

If households report disposing of trash by either 'composting', 'burning' or 'municipal garbage pickup' they are non-deprived. On the other hand, if the response is 'dump in forests/open land/rivers and streams' then they are deprived. Though landfills (municipal garbage pickup) and burning are considered adequate in the 2010 GNH Index, both do raise concerns regarding hazardous emissions and sustainability. Hence this item may be revised in the future.

Access to electricity is at the forefront of Bhutan's objectives. As a consequence significant rural electrification programmes have been initiated to provide electricity and to monitor more accurately the needs and the status of rural development (Tenth Five Year Plan, Royal Government of Bhutan 2009). The respondents are asked if their house has access to electricity and the threshold is set at 'yes' to access of electricity from grid.

The benefit of having an improved water supply to households and individuals is well known. Inadequate access to safe drinking water causes numerous illness and hygiene issues. It is also a source of socio-economic challenges since it requires resources to fetch water. The indicator developed here combines information on access to safe drinking water with information on the perceived quality of drinking water. The accessibility variable is defined by type of water facility source and ranges from piped water to rainwater. The quality of water is a subjective evaluation made by the respondents in terms colour, odour, taste etc.

In 2007, data from the Bhutan Living Standards Survey 2007 indicated that $90.9 \%$ of the population had access to an improved water source, leaving less than $10 \%$ of the people without access. The threshold used for access to water is based on the underlying concept laid down by UNICEF which defines an improved water source as a source that, by the nature of its design, protects the drinking water source from external contamination. As per the definition, an improved facility would include piped water into a dwelling, piped water outside of a house, a public outdoor tap or protected well. For the perceived quality of water, the threshold has been set to 'good' or 'very good'. Both conditions need to be fulfilled in order to be sufficient in water. The sufficiency for perceived water quality is $82 \%$ and the overall percentage of respondents sufficient in water is $81 \%$. 
Overall, a person is classified as having achieved sufficiency in service delivery if they enjoy sufficiency in each of the four elements. About $41 \%$ have achieved that condition.

\section{Government performance}

The indicator pertains to people's subjective assessment of the governments' efficiency in various areas. Although it is understood that objective indicators are valid measures of performance, subjective appraisals such as perceived performance quality can complement objective indicators (Van de Walle and Bouckaert 2003; Andrews, Boyne, and Walker 2006; Torenvlied and Akkerman 2009b). ${ }^{59}$ Justice must not only be done; it must be seen to be done. Similarly, in order for people to participate in governance, they must have some positive perception of governance overall. Furthermore, people may feel (and be) more secure if they believe (correctly) that the public institutions are working well.

To test people's perceptions of overall service delivery in the country, respondents are asked to rate the performance of the government in the past 12 months on seven major objectives of good governance: employment, equality, education, health, anti-corruption, environment and culture. These outcome-based questions enable respondents to rank the services on a five-point scale from 'very good' to 'very poor'. ${ }^{60,61}$ Principal component analysis shows a single factor to have loadings above 0.6 , and a high internal consistency was observed between the seven variables (Cronbach's alpha $=0.89$ ). The score can go as high as 35 indicating high performance and as low as 7 showing low performance of the government as perceived by people.

The people's perception of government performance is generally high. The mean score is $30(\mathrm{SD}=3.9)$ and median score is 31 . There is no variation

\footnotetext{
${ }^{59}$ Subjective data on performance of organisations are often criticised because people may not be sufficiently informed about policies or about a specific area that is put to question (Brown and Coulter 1983; Golden 1992; Kelly and Swindell 2002).

${ }^{60}$ There are numerous studies which have used different stages of performance indicators such as input, output, outcome etc. (Boyne and Law 1991; Sorber 1993; Duckett and Swerissen 1996; Hedley 1998; Stone and Cutcher-Hershenfeld 2001). A strong association between subjective and objective indicators for outcome performance indicators has been confirmed by Torenvlied and Akkerman (2009) in their multi-stage performance indicator research paper. For Bhutan, the performance index is based on outcome indicators.

${ }^{61}$ The response category also has the option of 'don't know' which has been re-categorised into mid-value 'average' which is considered a deprived category. This has no major impact on the results since individuals are expected to have some knowledge of the functioning of the institutions and so 'don't know' is inherently deprived.
} 
amongst age or literacy background. If the sufficiency threshold is set at 30 , this allows only one of the components to be rated 'average' or below - and 58.4\% are sufficient. However, there are possibilities of response bias because of the subjective nature of the statements and varying degree of knowledge among people in rural areas about the mentioned functions of governance which they may not observe. Hence, there needs to be some space for individuals who have limited knowledge either by the nature of their physical or mental conditions or a lack of information flow or personal experience. To include flexibility towards such issues, a slightly lower threshold is considered reasonable. A threshold of 28 was adopted, which means that a person has to perceive that public services are 'very good' or 'good' in at least five of the seven objectives. With this threshold, about $78.8 \%$ are considered to have achieved sufficiency.

\section{Community vitality}

The concept of GNH includes the social capital of the country, which is sustained through co-operative relationships and social networks within the community. A vital community can be described as a group of people who support and interact positively with other individuals and is based on a sense of cohesion amongst the members providing social support to one another. It is important to note that the concept outlined here reflects GNH values and Bhutanese moral beliefs.

A relevant definition of community was made in a working paper (McMillan 1976; Chavis 1983) for the Centre for Communities Studies. In the report, McMillian (1976) writes that a 'sense of community is a feeling that members have of belonging, a feeling that members matter to one another and to the group and a shared faith that members' needs will be met through their commitment to be together.'

From a GNH standpoint, a community must have strong relationships between community members and within families, must hold socially constructive values, must volunteer and donate time and/or money, and lastly must be safe from violence and crime. It is vital that volunteering and donations of time and money be recognized as a fundamental part of any community development. Socially constructive values can act as tools through which activities can be implemented for positive change in communities.

Empirical studies confirm that social capital affects people's learning and health (Fujiwara and Kawachi 2008) and also identify the community to be one of the significant determinants of wellbeing for individuals as well as families and communities (Putnam 1993, 2000; Field 2003). People who 
feel a sense of belonging tend to lead happier and healthier lives, and create more stable communities and a more supportive society (Zavaleta 2007). Social capital also has an instrumental value, increasing evidence shows that social cohesion is critical for societies to prosper economically and for development to be sustainable' (The World Bank 1999).

Therefore, it is only natural that a GNH society includes community vitality as one of the nine equally weighted domains. The indicators in this domain cover four major aspects of community: 1) social support which depicts the civic contributions made 2) community relationship, which refers to social bonding and a sense of community 3) family relationships, and 4) perceived safety. ${ }^{62}$

\section{Social support}

Social support here reflects the provision of support by volunteering or donating to an individual or a community. It is relevant to all spheres of life and without a doubt has a positive impact on a wide range of social, economic, cultural and environmental issues, including physical and mental wellbeing (David et al. 2008). It is understood that connectedness in a community is depicted in the strength of social networks within communities (Ura and Zangmo 2008), and it is volunteering and donating that encourages interactions between people and strengthens community connections. Involvement in volunteering and donation activities generates social capital, which creates a healthier and more vibrant community. In addition to these numerous benefits, it is also crucial for creating true partnerships between the different members of the community, business, NGOs and the government.

The giving of time and money - volunteering and donating - is a traditional practice in Bhutanese societies. These practices may have been more widespread in previous eras, because remote mountain communities depended on each other for survival. At the same time, commercialisation may devalue such traditional values which may lead to their decline. So it is vital to include these indicators to assess the level of social support in a community and trends across time.

To capture the rate of volunteering, respondents were asked for the number of days they volunteered and for the amount they donated. Donation is expressed in the total amount of financial resources donated

\footnotetext{
62 Similar concepts can be found in the following reports: Doolittle and McDonald 1978; Ahlbrandt and Cunningham 1979; Wandersman and Giamartino 1980; Riger and Lavrakas 1981; Bachrach and Zautra 1985; Davidson and Cotter 1986.
} 
in the past 12 months and volunteering is measured by the days donated in the past 12 months.

For donation, giving $10 \%$ of household income is considered sufficient, and for volunteering, three days per year is considered sufficient. The threshold has been identified based on normative grounds since there are no standards for minimum levels followed. In most cases, both conditions have to be satisfied to be classified as sufficient. This might mean, however, that a person who volunteered every spare hour but did not have enough money to give $10 \%$ was deemed insufficient; similarly, a senior businessperson who was tremendously generous and gave $30 \%$ of his or her income but could not manage to volunteer six days per year would be judged insufficient, as would an elderly person who was not physically or mentally able to volunteer. About $38 \%$ of respondents donate but do not volunteer. There are few who volunteer but do not donate - only $3 \%$. Most do both (51\%) and some choose to do neither (6.5\%). So, given the difference in the nature of donation (money and time) and the differences in the accompanying benefits, some compensation between these is allowed. In particular, if a person donates $20 \%$ of their income, then even if they do not volunteer it is considered sufficient and if they volunteer more than six days, but do not donate $10 \%$ of their income, it is still sufficient. With these conditions applied, overall $46 \%$ are sufficient.

\section{Community relationship}

A sustainable society requires strong social ties that bind people together in the community. In rural areas, people have a good sense of belonging, but urban areas communities are often described as having weaker social relationships as people have the ability to relocate themselves and their families depending on their choice of work, education and other personal events. Moreover, modernisation has created secular societies and already many have fast and busy lives, creating an environment conducive to isolation and detachment from their communities. This makes it harder for us to feel any sense of belonging to the neighbourhood and often leads to loneliness and depression.

Two variables have been considered: a sense of belonging and trust in neighbours. An increase in trust in one's neighbours is associated with better self-reported health status. ${ }^{63}$ The two components of the indicator

\footnotetext{
${ }^{63}$ This is true also for the GNH 2010 survey data: a chi square test revealed that there is an association between trust in neighbours with self-reported health status $(\mathrm{P}<0.05)$. One-way ANOVA results also indicate that there is a significant difference in the number of healthy days between groups who rated their trust levels differently $(\mathrm{P}<0.05)$. Higher trust had a higher number of healthy days and vice versa.
} 
are 'a sense of belonging' which ranges from 'very strong' to 'weak', and 'trust in neighbours' which ranges from 'trust most of them' to 'trust none of them'. Both indicators have options of 'don't know'. Seventy-one per cent have a very strong sense of belonging, $46 \%$ trust most of their neighbours, and $85 \%$ trust most or some of their neighbours.

GNH defines the notion of belonging or social identity as a central aspect of who we are. It is the strong and positive membership in families, communities or groups that ensures an individual's sense of belonging and so the thresholds here are based on normative reasons for sustaining and promoting a sense of community. The threshold for sense of belonging has been set at 'very strong', and for levels of trust 'some of them' and 'most of them' have been selected. The very few cases of 'don't know' have been identified as non-deprived, because respondents had just migrated to a community. For a person to have achieved sufficiency, both conditions have to be satisfied. The fulfilment of both conditions is necessary since a community must strive to create sense of connection derived from individuals' feelings of belonging as well as increased neighbourliness. When the threshold to both the indicators of community vitality is applied, $62.5 \%$ of people are sufficient in both.

\section{Family}

Good family relationships are vital for the health of family members as well as community members (Chophel 2010). For this indicator, six questions on a three-point scale of 'agree', 'neutral' and 'disagree' have been asked of the respondents. When aggregated, the family indicator score ranges from 6 to 18 . The mean score is $17(\mathrm{SD}=1.3)$ and median is 18. So, generally family relationships seem to be strong. This was also confirmed by one of the aspects of the life satisfaction indicator which described $95 \%$ as satisfied with their family relationships. It is a reasonable question to ask whether such positive response levels could have been biased by a fear that other family members were within earshot during the interview. The GNH interview sessions involved only the respondent and the interviewer so it is unlikely that responses were affected as other family members were not present or within earshot of the interview.

The underlying components of these questions have been tested through factor analysis. The test resulted in a single factor with loadings above 0.41 . They are added together to form an indicator with 18 as the maximum score (high family relationships) and 6 as the minimum score (low family relationships). The mean score was 17 (SD 1.3). 
It is clear that a GNH society considers family as one of the important determinants of an individual's wellbeing because relationships formed within family act as a positive force especially in a young person's life. So, naturally the highest possible score would be ideal for developing a society where families are a source of comfort, security and protection. However instead of using 18, a threshold of 16 is applied in order to allow 'neutral' responses in any two statements. Ninety-two per cent are satisfied in the family indicator.

\section{Victim of crime}

Feeling safe and secure at home, work and in the community is an essential prerequisite for sustaining a good quality of life. The neighbourhood is a critical environment for youth development (Parke and O'Neil 1999). The lack of neighbourhood safety also has a negative outcome on the health of the community members (Diprose 2007, De Jesu et al. 2010).64 Conversely, people who live in highly safe and supportive neighbourhoods have positive outcomes such as stronger connections with family, peers and community.

To assess safety in the community, respondents are asked whether they have been a victim of a crime in the past 12 months. The crime indicator has a simple two-point scale of 'yes' and 'no'. The threshold is set at 'no'. The crime statistics are low with only about $4 \%$ being described as victims. Self-reported victimisation however slightly underestimates victimisation when it concerns sexual offenses. In the next survey, other safety indicators might be incorporated to improve evaluation.

\section{Ecological diversity and resilience}

Bhutan has always recognized the central role environmental factors play in human development. Pursuant to Article 5 (Environment) of the Constitution of Bhutan, every Bhutanese citizen shall '... contribute to the protection of the natural environment, conservation of the rich biodiversity of Bhutan and prevention of all forms of ecological degradation including noise, visual and physical pollution....'

The environmental domain includes three subjective indicators related to perceptions regarding environmental challenges, urban issues and responsibilities, and one more objective question, related to wildlife

\footnotetext{
64 The study examined the associations between social networks and perceived neighbourhood safety among an ethnically diverse sample of 1352 residents living in lowincome public housing sites in Boston, Massachusetts.
} 
damage to crops. Indicators in this domain in particular may be reconsidered for future GNH surveys.

Perception of environmental issues in general and how they vary across time has long been of interest to researchers and policymakers. ${ }^{65}$ However, there are concerns that hamper the use of individual-level and subjective indicators for ecology since they fail to consider the full complexity of the ecological system. Thus, ideally, future GNH surveys would incorporate other indicator(s) or data from localised environmental sources. In the absence of such indicator(s) subjective indicators were used as proxy measures for changes in environmental issues across time. However like other subjective indicators, the interpretation of these indicators is clouded by different and possibly shifting frames of reference, so they are given a light weight of $10 \%$ of the environmental domain each.

\section{Pollution}

The awareness and knowledge of citizens about their environment are crucial for pro-environmental actions and for making environmental policies successful. At the same time, the aim is to ensure that serious environmental issues are redressed. So, in order to test people's environmental awareness, a series of questions were developed to test the perceived intensity of environmental problems. The expressions of environmental concern were aimed at understanding people's concerns, knowledge and awareness of environmental conditions in their respective communities. Many studies use the results of perception data as a proxy for the level of environmental awareness (Eurobarometer 1992; Gallup 2011; Iizuka 2000; Inter-university Consortium for Political and Social Research (ICPSR) 1993). In most cases, these were carried out as timeseries studies of environmental public opinion while only a limited number were cross-national opinion surveys of environmental awareness for developing countries (United Nations Environmental Programme and Harris 1989). In both cases, the studies were successful in pointing out the existence of concerns among the general public (Iizuka 2000). Further, it was observed that an examination of environmental attitudes seems feasible particularly in a low-income setting (White and Hunter 2005).

Seven environmental issues of concern were shared with respondents, and their responses follow a four-point scale from 'major concern' to 'not a concern'. Principal component analysis resulted in two factors but loadings were observed to be high for all variables in the first factor $(>0.62)$. The

\footnotetext{
${ }^{65}$ However, perceptions of environmental issues are affected by the extent to which people are informed on those issues (McGowan and Sauter 2005).
} 
alpha coefficient was 0.85 . The pollution indicator has a high score of 32 and low score of 8 .

Issues that were frequently reported as major concerns were landslides $(28 \%)$, littering $(26 \%)$, soil erosion $(25 \%)$ and floods $(25 \%)$. Note that these percentages are not region specific. For instance, if littering were studied regionally then about $40 \%$ of urban dwellers consider littering as a 'major concern' while 19\% from rural areas report the same. Similarly, options of 'don't know' are allowed, which is considered as deprived since it is indicative of a lack of environmental knowledge. They are not added into a single number but rather a conditional threshold is applied whereby an individual is insufficient if he or she has rated 'major concern' or 'some concern' in at least five of the seven environmental issues. Their reference frame is within the past 12 months; however, as with many subjective indicators, there might be errors with the reference frame and so it is not very practical to give more weight to perceptive data by fixing high thresholds. Hence, with the proposed threshold, $69 \%$ are sufficient in the pollution indicator.

\section{Environmental responsibility}

Having the right attitude towards the environment is fundamentally important and also a widely discussed topic. ${ }^{66}$ The indicator attempts to measure the feelings of personal responsibility towards the environment. It is crucial to reinforce attitudes that will encourage people to adopt ecofriendly approaches and also to identify any deterioration in the current very environmentally aware views of citizens. The responses run on a fourpoint scale ranging from 'highly responsible' to 'not at all responsible'. When the threshold is set at 'highly responsible', $84.4 \%$ are sufficient.

\section{Wildlife}

While wildlife is a valuable natural resource with several beneficial values to people and the ecosystem, it can cause damage to society in terms of livestock and crop loss, attacks on people and infrastructure. The wildlife indicator here incorporates information on damage to crops. There has been a growing concern about wildlife damage to crops in Bhutan (Choden and Namgay 1996; Wang, Curtis and Lassoie 2006). Wildlife damage can have catastrophic economic consequences for farmers, especially for vulnerable households; it also disrupts sleep patterns and may create anxiety and insecurity. Therefore, an assessment of the scale

\footnotetext{
${ }^{66}$ Environmental attitudes have frequently been measured since the 1970s (Rokeach 19601979; McGuire 1969; Bem 1970; Fishbein and Ajzen 1975; Dunlap and Van Liere 1978).
} 
and extent of damage in the fields is essential for decision making on wildlife management and crop protection. The current data show that crop depredation by wildlife is a substantial concern to farmers with only $21 \%$ reporting 'no' wildlife damage in the past 12 months.

To assess the exact extent of damage, it would be necessary to examine every field. Even when investigated immediately, the true cost of damage may not be easy to ascertain. For example, it may be difficult to distinguish damage caused by different animals (Van Eerden 1990; Hötte and Bereznuk 2001). To address this uncertainty, there is a need for sound ecological research to quantify animal-inflicted damage and economic losses in relation to other sources of damage (e.g. Thirgood and Redpath 2008). However, this is not practical for this survey, which has to incorporate other indicators. So a simple self-reported estimate is used as a proxy for the quantitative assessment. Two simple questions on the presence and absence of damage and the severity of damage are applied to determine the impact of wildlife damage on agriculture. Moreover, understanding perceptions of farmers regarding wildlife damage to crops is a critical element since they are the key stakeholders in the process. The indicators hope to promote a better understanding of wildlife damage and thereby help advance or provide skills and knowledge for wildlife damage management practices.

The first question deals with whether respondents consider it as a constraint to farming. Responses are given on a four-point scale ranging from 'major constraint' to 'not a constraint'. The threshold has been set at 'minor constraint'. The second indicator pertains to the severity of damage, i.e. crop loss. Respondents are asked to provide an average perceived amount of crop lost, if the crop had been damaged by wildlife. It ranges from 'a lot' to 'not at all'. For both the indicators the reference frame is the past 12 months.

The threshold is fixed such that respondents are deprived if they report either 'some constraint' or 'major constraint' and account for a crop loss of 'a lot' or 'some'. Although the indicators measure the same thing conceptually, human perceptions may be distilled from long and short memories from distant associates and so, two are preferred as one may reaffirm the claim made by the other. For example, 51\% claim that wildlife was a 'major constraint' in the past 12 months while $21 \%$ report it as 'some constraint'. Note that it includes respondents who are farmers only. Thirty-eight per cent of farmers report that 'a lot' of their crops have been damaged and 25\% report 'some' damage. Together, 37\% report 'major constraint' and 'a lot' of damage while $2 \%$ of farmers report 'major constraint' but 'no' damage was done to their crops. The lack of actual 
numeric amounts or percentages of actual crop loss may give rise to errors. So, to allow for such inconsistencies, both conditions have to be fulfilled. In future surveys, perhaps assigning a numeric value to the amount of crop loss would be more accurate. With the threshold, $57.9 \%$ of the respondents attain sufficiency. Note the wildlife indicator is rural specific since it pertains to farmers. Individuals from other occupational backgrounds such as civil servants or corporate workers are classified as non-deprived. The rural-specific indicator is later offset by the urbanissues indicator which in turn applies to urban dwellers only. It must also be understood that wildlife damage might result in people having negative attitudes towards conservation. Hence, care must be taken in analysis of the results.

\section{Urban issues}

Bhutan is undergoing rapid urbanisation resulting in the growth of city and town populations. Urban growth has a number of positive impacts on human wellbeing such as improvements in energy, health care, infrastructure and services. Despite these positive impacts, increasingly there are environmental issues faced by almost all major cities. Some of the major issues in developing countries are traffic congestion, inadequate green spaces, urban sprawl, etc. Since these issues have adverse impacts on wellbeing, it is crucial to incorporate them into the GNH Index.

Respondents are asked to report their worries about four urban issues: traffic congestion, inadequate green spaces, lack of pedestrian streets and urban sprawl.

The threshold is set such that a person can report any one of the issues as major threat or worry to be sufficient. About $84.4 \%$ achieve sufficiency; this is in part because people who live in rural areas have been automatically classified as sufficient to offset the wildlife damage indicator introduced above. This indicator mainly acts as a proxy for sustainable urban development which is one of the major objectives of the government.

\section{Living standards}

The living standards domain refers to the material wellbeing of the Bhutanese people. It ensures the fulfilment of basic material needs for a comfortable living. Over the years, the material standard of living has risen steadily due to advances in development. However, about 23.2\% (Royal Government of Bhutan 2007) of Bhutanese still live in income poverty; some lack assets such as land or adequate housing. 
There is a wide range of indicators used in the literature to assess standards of living. For individual-level analysis, the actual consumption of food or other goods and services like health and education is often argued to be the most accurate. In practice, income and expenditure levels are often used since individual consumption is difficult to disaggregate. Here, we use three indicators to assess people's standards of living: household per capita income, assets and housing conditions. Assets include livestock, land and appliances, while housing conditions are measured by room ratio, roofing and sanitation. These are included so that there are enough complementary measures for self-reported household income.

\section{Housebold income}

Household income includes income earned by all the individuals in a household from varied sources within or outside of the country. The household income here has been adjusted for in-kind payments received.

In the literature, two types of thresholds are generally used, either a fixed threshold like a poverty line or relative thresholds such as mean or median income. The poverty line for Bhutan is Nu. 1,096.94 per person per month in the Poverty Analysis Report (Royal Government of Bhutan 2007).67 The mean household per capita was generated by dividing the household income by household size, without equivalence scales. In Bhutan Living Standards Survey (BLSS) (2007) it was Nu. 31834.3. When a poverty line threshold (Nu. 1,096.94) was used on individual income, the headcount estimation made by the Poverty Analysis Report (Royal Government of Bhutan 2007) was $23.2 \%$.

For the GNH Index, it would not be sensible to use the poverty line as a threshold because the threshold should reflect sufficient income. The GNH living standards domain refers to higher conditions for wellbeing than poverty lines. One option would be to use a relative income threshold for the sufficiency threshold as is commonly done in European countries. Thresholds like $60 \%$ of the median or $50 \%$ of mean income are often used to identify poverty. ${ }^{6}$

Yet for the GNH indicator an absolute sufficiency threshold was chosen, since the GNH values encourages people to achieve happiness through their accomplishments, and discourages a relative approach in which one is satisfied only if one has relatively more income (or other achievements)

\footnotetext{
${ }^{67}$ The poverty line given here is a measure for absolute poverty developed by the National Statistical Bureau of Bhutan in 2007 and is based on food and non-food needs.

68 See for example, Gordon (2006) and Hillyard et al. (2003).
} 
than one's peers. In this regard, a threshold is computed from a GNH data- adjusted poverty line ${ }^{69}$ by multiplying the national poverty line by 1.5 . It would have amounted to $\mathrm{Nu} .14,200$ per person per year in the BLSS 2007 data.

There were particular challenges in the measurement of income sufficiency. The questionnaire for income and expenditure in the GNH Survey differed from the BLSS, and the resulting GNH data had different median and mean values from the BLSS data as well as different district rankings by both poverty and average per capita income. As a result, in the income indicator alone, we implemented the poverty threshold of 1.5 times the poverty line in the original BLSS 2007 dataset to obtain the percentage of people who enjoyed sufficiency in income. We then mapped the same percentage onto the GNH income per capita data, identifying those having the highest income as enjoying sufficiency. This will be modified in the next GNH survey. For if the GNH income data are from a different distribution, then the identification of a given percentage of the income poorest may still be inaccurate. Furthermore, in using the percentage from BLSS data we are assuming that the percentage of people who enjoy 1.5 times the poverty line in 2010 is the same as those in 2007 . This assumption is likely to underestimate the percentage of people enjoying sufficiency. The income threshold classifies $54 \%$ of people as sufficient.

\section{Assets}

An asset indicator has been used as an indicator of living standards in many studies (Montgomery et al. 2000; Morris et al. 2000; Filmer and Pritchett 2001; Case et al. 2005). ${ }^{70}$ The indicator uses data on selected household assets, such as durable and semi-durable goods of everyday use, to describe household welfare. The concept is based on evidence that income/expenditure measures are incomplete measures of the material wellbeing of households especially in developing countries where such data may have higher measurement errors. The studies found that the asset indicator was robust, produced internally coherent results, and was consistent with financial means. Further, asset data were found to be

\footnotetext{
${ }^{69}$ The GNH data poverty line has been adjusted for the difference in the medians between BLSSR data and GNH data. Poverty line for GNH data = Poverty line (PAR 2007)*Median (BLSSR data)/Median (GNH data)

70 The asset index developed by Filmer and Pritchett (1999) has been used in Demographic and Health Surveys (DHS) to estimate reasonable wealth effects.
} 
more reliable and easier to collect. ${ }^{71}$ However, it is necessary to be aware that the indicator provides a relative analysis of welfare and little about levels of absolute income. The items of the indicator are taken from a generic list of goods, the uses of which may not be the same across all household members, and quality aspects of the goods owned were not included.

Commonly, asset indicators are defined by appliances such as a mobile phone, radio or TV or bicycle; however, because of the socio-cultural context, livestock and land ownership were also considered assets. Livestock is understood as an integral component in agricultural and rural economies in Bhutan. Most farming is still subsistence farming, and the difficult terrain makes it challenging to use modern equipment. Thus, the work must be done by animals and humans. Moreover, animals provide households with transport, fertilizer and food and also employment. So, they are critical assets especially for poor households. Similarly, land ownership is particularly relevant for rural agricultural-based economies. In some of the focus group participants' perceptions, a decent living standard always included livestock and land ownership. ${ }^{72}$

The asset indicator is created from three major components: 1) appliances (mobile phone, fixed-line telephone, personal computer, refrigerator, colour television and washing machine) 2) livestock ownership and 3) land ownership.

The thresholds are applied at two levels: they are set initially on each of the three indicators and then later, an overall threshold is applied to classify insufficiency in the asset indicator.

For a measure of appliances, a series of household items that could be considered amenities for the family was developed. Principal component analysis has been used to determine the selection of appliances. The first factor explained $80 \%$ of the variance and contained six appliances mobile phone, fixed-line phone, personal computer, refrigerator, washing machine and colour television. The mobile phone could be dropped from the list of appliances since, in a general sense, the utility is marginal and limited to the one who owns it. For the other appliances, the scope of

\footnotetext{
${ }^{71}$ Enumerators of the GNH surveys pointed out that the asset index was more accurate since it's easier for respondents to reflect on their ownership than on income. Additionally, enumerators could confirm the ownership by actually seeing goods in the household. So, the asset index is less likely to contain reporting bias.

72 The analysis is based on the focus group discussions conducted by Dr. Alkire, Tshoki Zangmo and Tshering Phuntsho in Wangdue Phodrang and Punakha in 2011.
} 
functional utility is much wider and other members of the household might have access. However, in rural areas if a household owned a mobile phone then that would imply that every household member had some access to it. Moreover, fixed-line phones are being replaced by mobile phones even in urban areas; only $21 \%$ of urban households now have fixed-line phones. So, in the end, all six items loaded in the first factor were considered for the asset indicator. The sufficiency threshold was set to three, and $31 \%$ are sufficient in appliances.

It is widely known that livestock constitute an important source of income, especially in rural areas and nomadic areas of the country. They contribute to a household's livelihood by providing cash income or in-kind income through the sale of animal products or the animals themselves and thereby act as savings for future security. Although the importance of including livestock as an asset is generally agreed upon, setting a threshold becomes challenging because of the difference in the capital and maintenance costs of different species, which are usually higher for larger ruminants. Larger ruminants require more fodder while smaller domestic animals, such as chickens, can survive on a lesser amount. And so, based on the rates of an average domestic purchase, a threshold is defined. It was observed that an average price of 40 chickens would be equivalent to the average rate of others. Ownership of chickens has been reclassified accordingly. In terms of thresholds, Bhutan's national MPI (2010) sets it at three, but for the GNH Index it has to be set higher. And so, livestock has been set to five normatively. About $41.3 \%$ of the respondents are sufficient in livestock.

The data on land were collected in the categories of dry land and (unterraced); wet land (irrigated and terraced); pan₹bing, which is a type of land use in which land is cultivated after leaving it fallow to improve soil fertility; orchards; kitchen gardens; and tseri, which refers to shifting cultivation. Although the Land Act of 2007 banned tseri cultivation, the survey shows about $14.4 \%$ of the respondents still practice it. The average land holding is 2.9 acres per household ( $\mathrm{SD}=3.6$ ). The average rural land holding is 3.39 acres per rural household, and for urban areas it is 0.86 acre per household.

In setting the sufficiency cutoff for land, there are numerous factors that need to be taken into consideration such as the quality of land, household size, area and type of farming practices and sources of other income. The household size plays a role as smaller families might require smaller land holdings and larger families might need more land. The region of location is also a huge determinant since an agriculture-based economy usually requires more land holdings. Lastly, the type of farming must also be considered, for instance whether the land is being used for crops or orchards or just as pasture for animals and also whether the particular 
household has other sources of income. Given the wide range of factors that require equal attention, it is challenging to set a threshold that fulfils all these conditions.

The focus group discussions carried out in some districts concluded that five acres was the threshold for a rural farming household with an average family size of five. It was decided that for farming-related activities an average of five acres would be sufficient to grow crops or fruits or for livestock management. The land asset is included to reflect assets for rural areas, and so understanding land ownership in rural areas is pertinent for setting the threshold. In rural areas, only $26 \%$ of households have five or more acres of land, while about $44 \%$ have three or more acres of land. For the MPI Bhutan 2010, the threshold was set to one acre, but the GNH Index is not a poverty measure and so a minimum threshold cannot be applied. The average household size in rural areas is 4.7 , and the sufficiency threshold for an average land amount was normatively set to five acres. About $22 \%$ are sufficient; however, note that the GNH also includes urban dwellers whose income comes mostly from employment, so they would be regarded as deprived in this sub-indicator (but not necessarily overall as we see below).

The final threshold across the three assets is applied so that if a household possesses sufficiency in either appliances or livestock or land then the household is classified as being sufficient in assets overall. This implies that any one condition of the three can be satisfied to be in order to be labelled non-deprived. This threshold was selected based on its flexibility to incorporate individuals from diverse occupational backgrounds, as well as from varied areas of residence. For example, livestock and farm land may not be very relevant to a person who is employed in a service occupation but may be particularly valid in remote areas. It must be understood that the objective of an asset indicator is to supplement information on income with some crude indicator of wealth. Asset indices may move more slowly than income and expenditure. This gives rise to data reliability issues for GNH Index analysis that attempts to capture trends in wellbeing over time. This requires not only that we interpret results with due caution but that we also keep in mind the complexities of combining the three assets together. However given the issues with the income data mentioned above, both indicators were included to improve accuracy. Application of the overall conditional threshold identifies $74.1 \%$ of Bhutanese to have achieved sufficiency. 


\section{Housing quality}

The domain is incomplete without including an indicator of housing conditions. The benefits of good housing can be observed from both an individual as well as from a community perspective. On the individual level, having one's personal space is considered fundamental for one's biological, psychological and social needs since it is a place where most spend a significant part of their everyday lives. ${ }^{73}$ Studies show the critical impacts that poor quality, overcrowded and temporary accommodation can have on an individual's physical and mental health. ${ }^{74}$ From a community standpoint, aspects such as combating social exclusion and discrimination and strengthening social cohesion cannot be achieved unless there are proper living spaces and a decent standard of accommodation. Overcrowded accommodation, which is based on the number of rooms and number of household members, can lead to family disintegration, weakening community ties and is considered to give rise to a variety of social ills. Therefore, insufficient housing conditions can pose a threat to not only the wellbeing of individuals but also the community at large.

The quality of housing is composed of three indicators: the type of roofing, type of toilet and room ratio. The thresholds have been set based on the Millennium Development Goals such as corrugated galvanized iron (CGI) or concrete brick or stone for roofing, pit latrine with septic tank for toilet and two persons per room for overcrowding, and all three conditions must be met. So, overall an individual is sufficient in housing if he or she lives in a house that has a good roofing structure (CGI or concrete brick or stone), a pit latrine with a septic tank and uncrowded rooms. In reality, having a higher quality roof may by far outweigh toilet condition as far as housing quality is considered. With the stated threshold, about $46.2 \%$ are sufficient in housing quality.

\footnotetext{
${ }^{73}$ Many studies have confirmed that good housing is at the top of the hierarchy of human needs (Burns and Grebler 1986; Kiel and Mieszkowski 1990).

${ }^{74}$ These are just some of the studies that show the impact of housing quality on welfare. For example, Housing, Health and Climate Change: Developing Guidance for Health Protection in the Built Environment: Mitigation and Adaptation Responses, World Health Organisation (2010)
} 


\section{Appendix 6: Additional Tables}

Table I: Main GNH Results This table provides the at-a-glance information on the GNH Index.

Suggested citation: Ura, Karma; Alkire, Sabina; and Zangmo, Tshoki (Nov 2011) www.grossnationalhappiness.com ophi.qeh.ox.ac.uk

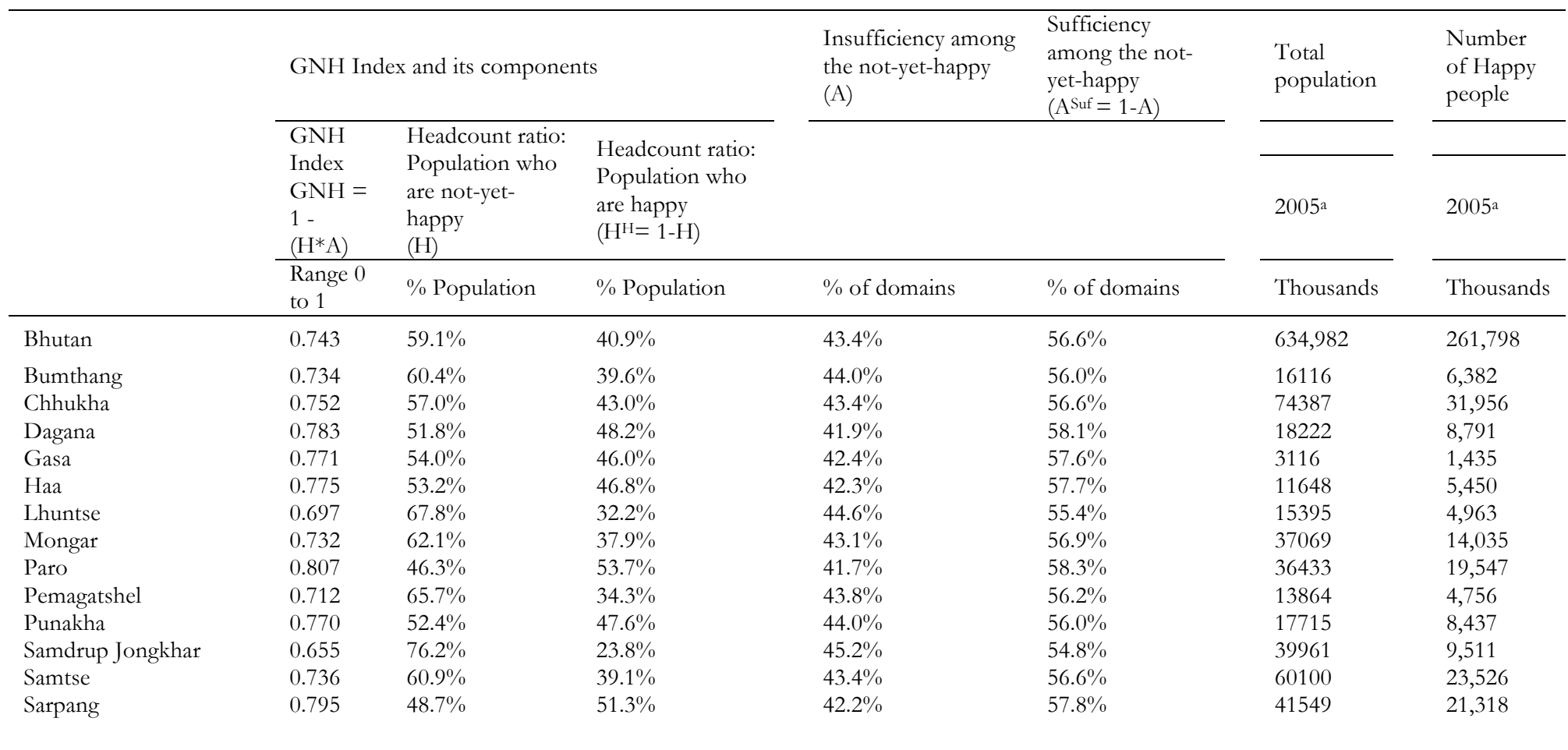


Karma Ura, Sabina Alkire, Tshoki Zangmo \& Karma Wangdi

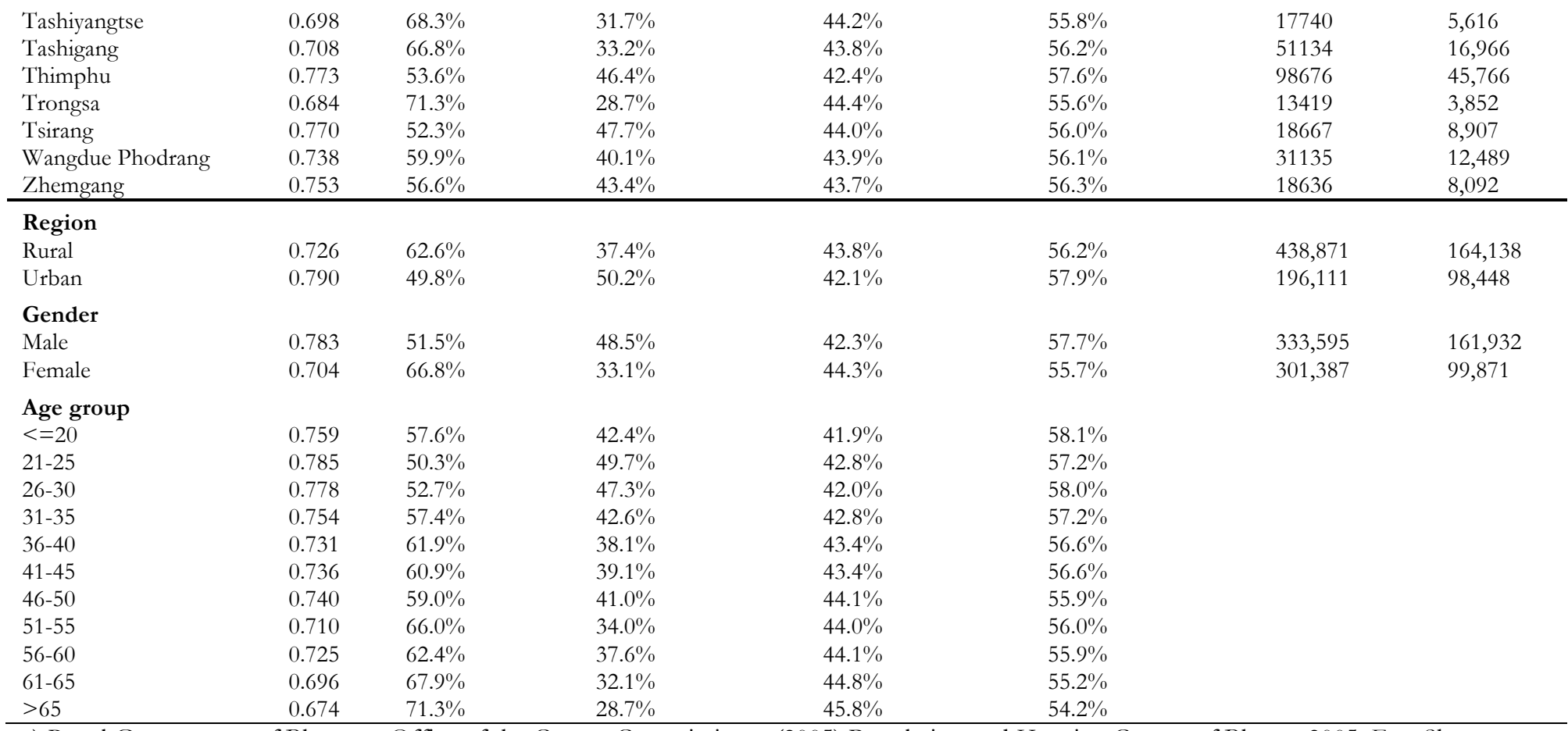

a) Royal Government of Bhutan - Office of the Census Commissioner (2005) Population and Housing Census of Bhutan 2005. Fact Sheet,

Thimphu: Office of the Census Commissioner, p. 2 [available at http://www.bhutancensus.gov.bt/Fact_sheet.pdf] 
An Extensive Analysis of GNH Index

Table II-A Understanding Happiness - Composition \& Contributions The table shows the percentage contribution to overall happiness.

Suggested citation: Ura, Karma; Alkire, Sabina; and Zangmo, Tshoki (Nov 2011) www.grossnationalhappiness.com ophi.qeh.ox.ac.uk

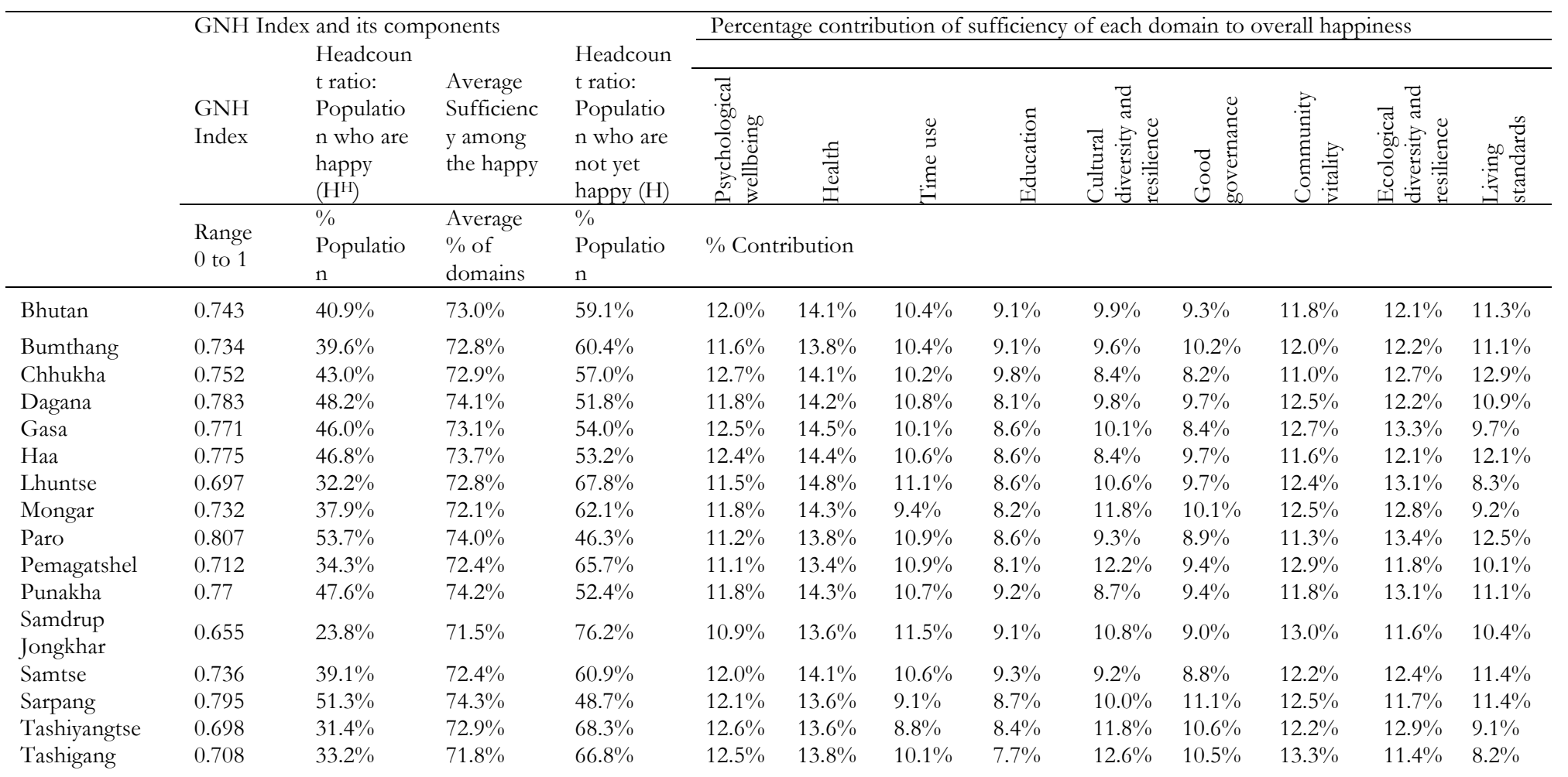


Karma Ura, Sabina Alkire, Tshoki Zangmo \& Karma Wangdi

\begin{tabular}{|c|c|c|c|c|c|c|c|c|c|c|c|c|c|}
\hline Thimphu & 0.773 & $46.4 \%$ & $72.5 \%$ & $53.6 \%$ & $11.8 \%$ & $14.6 \%$ & $10.8 \%$ & $10.7 \%$ & $9.2 \%$ & $8.6 \%$ & $9.8 \%$ & $11.1 \%$ & $13.4 \%$ \\
\hline Tsirang & 0.77 & $47.7 \%$ & $73.8 \%$ & $52.3 \%$ & $11.8 \%$ & $13.5 \%$ & $10.2 \%$ & $8.9 \%$ & $10.4 \%$ & $9.8 \%$ & $12.8 \%$ & $11.4 \%$ & $11.2 \%$ \\
\hline $\begin{array}{l}\text { Wangdue } \\
\text { Phodrang }\end{array}$ & 0.738 & $40.1 \%$ & $72.3 \%$ & $59.9 \%$ & $12.7 \%$ & $14.3 \%$ & $11.0 \%$ & $8.1 \%$ & $9.8 \%$ & $9.8 \%$ & $12.3 \%$ & $11.3 \%$ & $10.8 \%$ \\
\hline \multicolumn{14}{|l|}{ Region } \\
\hline Rural & 0.726 & $37.4 \%$ & $73.0 \%$ & $62.6 \%$ & $12.1 \%$ & $13.9 \%$ & $10.5 \%$ & $8.4 \%$ & $10.5 \%$ & $9.7 \%$ & $12.7 \%$ & $12.2 \%$ & $10.0 \%$ \\
\hline Urban & 0.790 & $50.2 \%$ & $72.7 \%$ & $49.8 \%$ & $11.7 \%$ & $14.4 \%$ & $10.4 \%$ & $10.4 \%$ & $8.7 \%$ & $8.5 \%$ & $10.1 \%$ & $11.9 \%$ & $13.9 \%$ \\
\hline \multicolumn{14}{|l|}{ Age group } \\
\hline$<=20$ & 0.759 & $42.4 \%$ & $72.8 \%$ & $57.6 \%$ & $10.7 \%$ & $14.3 \%$ & $12.6 \%$ & $10.8 \%$ & $8.5 \%$ & $8.2 \%$ & $10.6 \%$ & $13.0 \%$ & $11.3 \%$ \\
\hline $21-25$ & 0.785 & $49.7 \%$ & $72.6 \%$ & $50.3 \%$ & $10.7 \%$ & $14.4 \%$ & $11.7 \%$ & $10.3 \%$ & $9.7 \%$ & $8.6 \%$ & $10.8 \%$ & $12.2 \%$ & $11.6 \%$ \\
\hline $26-30$ & 0.778 & $47.3 \%$ & $73.0 \%$ & $52.7 \%$ & $11.4 \%$ & $14.3 \%$ & $10.8 \%$ & $10.2 \%$ & $9.1 \%$ & $8.4 \%$ & $11.1 \%$ & $12.5 \%$ & $12.1 \%$ \\
\hline $31-35$ & 0.754 & $42.6 \%$ & $72.4 \%$ & $57.4 \%$ & $12.0 \%$ & $14.1 \%$ & $9.8 \%$ & $9.6 \%$ & $9.9 \%$ & $9.1 \%$ & $11.6 \%$ & $12.5 \%$ & $11.4 \%$ \\
\hline $36-40$ & 0.731 & $38.1 \%$ & $73.5 \%$ & $61.9 \%$ & $12.3 \%$ & $14.0 \%$ & $9.6 \%$ & $9.3 \%$ & $10.0 \%$ & $9.2 \%$ & $12.0 \%$ & $11.7 \%$ & $11.8 \%$ \\
\hline $61-65$ & 0.696 & $32.1 \%$ & $73.4 \%$ & $67.9 \%$ & $13.3 \%$ & $13.0 \%$ & $10.8 \%$ & $6.4 \%$ & $11.2 \%$ & $10.3 \%$ & $13.1 \%$ & $11.9 \%$ & $10.0 \%$ \\
\hline$>65$ & 0.674 & $28.7 \%$ & $72.1 \%$ & $71.3 \%$ & $13.5 \%$ & $13.3 \%$ & $11.4 \%$ & $6.0 \%$ & $10.8 \%$ & $10.4 \%$ & $12.5 \%$ & $11.8 \%$ & $10.3 \%$ \\
\hline
\end{tabular}


An Extensive Analysis of GNH Index

Table II-B Understanding Happiness - Composition \& Contributions The table shows the percentage contribution to overall happiness.

Suggested citation: Ura, Karma; Alkire, Sabina; and Zangmo, Tshoki (Nov 2011) www.grossnationalhappiness.com ophi.qeh.ox.ac.uk

\begin{tabular}{|c|c|c|c|c|c|c|c|c|c|c|c|c|c|c|c|c|c|c|}
\hline & \multicolumn{3}{|c|}{$\begin{array}{l}\text { Psychological wellbeing } \\
\text { indicators }\end{array}$} & \multicolumn{3}{|c|}{ Health } & \multirow[b]{2}{*}{ 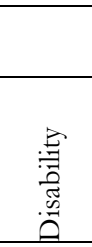 } & \multirow[b]{2}{*}{ 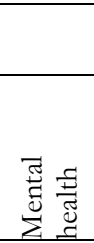 } & \multicolumn{2}{|c|}{ Time use } & \multicolumn{3}{|c|}{ Education } & \multicolumn{5}{|c|}{ Cultural diversity and resilience } \\
\hline & 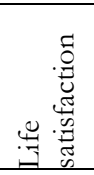 & 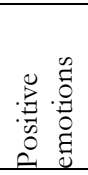 & 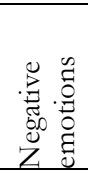 & $\begin{array}{l}\text { 量 } \\
\text { 营 } \\
\text { 营 }\end{array}$ & 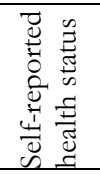 & 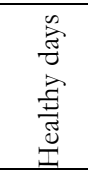 & & & $\frac{M}{3}$ & $\frac{\ddot{\ddot{U}}}{\omega}$ & 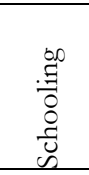 & 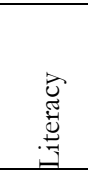 & $\frac{\mathscr{n}}{\frac{\pi}{\pi}}$ & $\begin{array}{l}80 \\
\frac{8}{0} \\
\frac{U}{E} \\
0 \\
E\end{array}$ & 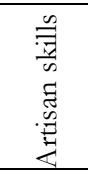 & 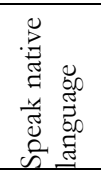 & 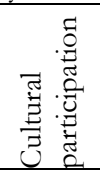 & 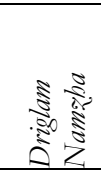 \\
\hline & \multicolumn{18}{|c|}{ Percentage Contribution } \\
\hline Bhutan & $4.9 \%$ & $1.8 \%$ & $1.9 \%$ & $3.4 \%$ & $1.3 \%$ & $4.0 \%$ & $4.4 \%$ & $4.4 \%$ & $4.9 \%$ & $5.5 \%$ & $2.1 \%$ & $2.6 \%$ & $3.7 \%$ & $0.6 \%$ & $3.2 \%$ & $2.9 \%$ & $2.0 \%$ & $1.8 \%$ \\
\hline Bumthang & $4.9 \%$ & $1.8 \%$ & $1.7 \%$ & $3.2 \%$ & $1.4 \%$ & $3.9 \%$ & $4.2 \%$ & $4.3 \%$ & $4.4 \%$ & $6.0 \%$ & $2.1 \%$ & $2.8 \%$ & $3.7 \%$ & $0.5 \%$ & $3.4 \%$ & $3.0 \%$ & $2.2 \%$ & $1.0 \%$ \\
\hline Chhukha & $5.0 \%$ & $1.9 \%$ & $2.1 \%$ & $3.6 \%$ & $1.4 \%$ & $4.0 \%$ & $4.5 \%$ & $4.2 \%$ & $5.4 \%$ & $4.9 \%$ & $2.6 \%$ & $2.9 \%$ & $3.7 \%$ & $0.6 \%$ & $2.9 \%$ & $3.0 \%$ & $0.9 \%$ & $1.7 \%$ \\
\hline Dagana & $4.6 \%$ & $2.0 \%$ & $2.0 \%$ & $3.2 \%$ & $1.2 \%$ & $4.1 \%$ & $4.4 \%$ & $4.4 \%$ & $5.2 \%$ & $5.6 \%$ & $1.7 \%$ & $2.5 \%$ & $3.6 \%$ & $0.3 \%$ & $3.5 \%$ & $2.9 \%$ & $1.7 \%$ & $1.7 \%$ \\
\hline Gasa & $4.9 \%$ & $2.0 \%$ & $2.2 \%$ & $3.4 \%$ & $1.3 \%$ & $4.3 \%$ & $4.4 \%$ & $4.4 \%$ & $4.0 \%$ & $6.1 \%$ & $2.0 \%$ & $2.3 \%$ & $3.8 \%$ & $0.6 \%$ & $3.3 \%$ & $3.0 \%$ & $1.9 \%$ & $1.9 \%$ \\
\hline Наa & $5.0 \%$ & $2.0 \%$ & $2.1 \%$ & $3.2 \%$ & $1.4 \%$ & $4.3 \%$ & $4.4 \%$ & $4.3 \%$ & $5.0 \%$ & $5.6 \%$ & $1.8 \%$ & $2.5 \%$ & $3.7 \%$ & $0.7 \%$ & $2.5 \%$ & $3.0 \%$ & $1.5 \%$ & $1.5 \%$ \\
\hline Lhuntse & $4.8 \%$ & $1.4 \%$ & $1.8 \%$ & $3.4 \%$ & $1.4 \%$ & $4.4 \%$ & $4.5 \%$ & $4.5 \%$ & $4.4 \%$ & $6.8 \%$ & $1.6 \%$ & $2.5 \%$ & $3.7 \%$ & $0.7 \%$ & $3.3 \%$ & $3.0 \%$ & $2.3 \%$ & $2.0 \%$ \\
\hline Mongar & $5.0 \%$ & $1.5 \%$ & $2.1 \%$ & $3.2 \%$ & $1.3 \%$ & $3.9 \%$ & $4.5 \%$ & $4.6 \%$ & $3.8 \%$ & $5.5 \%$ & $1.5 \%$ & $2.2 \%$ & $3.8 \%$ & $0.6 \%$ & $3.7 \%$ & $3.1 \%$ & $2.7 \%$ & $2.4 \%$ \\
\hline Paro & $4.8 \%$ & $1.6 \%$ & $1.5 \%$ & $3.3 \%$ & $1.3 \%$ & $3.9 \%$ & $4.2 \%$ & $4.3 \%$ & $5.6 \%$ & $5.3 \%$ & $2.2 \%$ & $2.5 \%$ & $3.5 \%$ & $0.4 \%$ & $2.1 \%$ & $3.0 \%$ & $2.4 \%$ & $1.8 \%$ \\
\hline Pemagatshel & $4.6 \%$ & $1.5 \%$ & $1.9 \%$ & $3.1 \%$ & $1.3 \%$ & $3.9 \%$ & $4.2 \%$ & $4.1 \%$ & $4.1 \%$ & $6.8 \%$ & $1.6 \%$ & $2.5 \%$ & $3.8 \%$ & $0.2 \%$ & $3.9 \%$ & $3.1 \%$ & $2.9 \%$ & $2.3 \%$ \\
\hline Punakha & $4.8 \%$ & $2.0 \%$ & $1.8 \%$ & $3.1 \%$ & $1.4 \%$ & $4.3 \%$ & $4.4 \%$ & $4.2 \%$ & $5.4 \%$ & $5.3 \%$ & $2.0 \%$ & $2.7 \%$ & $3.7 \%$ & $0.8 \%$ & $2.4 \%$ & $3.0 \%$ & $1.8 \%$ & $1.5 \%$ \\
\hline $\begin{array}{l}\text { Samdrup } \\
\text { Jongkhar }\end{array}$ & $4.5^{\circ}$ & $1.6 \%$ & 1.5 & 0 & 1 & 0 & $4.4 \%$ & 4 & 5.1 & 6 & $\%$ & $\%$ & $\%$ & $0.5 \%$ & $3.5 \%$ & $2.9 \%$ & $2.6 \%$ & $1.8 \%$ \\
\hline Samtse & $5.0 \%$ & $1.7 \%$ & $2.1 \%$ & $3.2 \%$ & $1.1 \%$ & $3.9 \%$ & $4.5 \%$ & $4.6 \%$ & $5.2 \%$ & $5.4 \%$ & $2.3 \%$ & $2.8 \%$ & $3.8 \%$ & $0.5 \%$ & $3.1 \%$ & $2.8 \%$ & $1.2 \%$ & $2.0 \%$ \\
\hline Sarpang & $4.8 \%$ & $2.1 \%$ & $1.9 \%$ & $3.3 \%$ & $1.0 \%$ & $4.1 \%$ & $4.3 \%$ & $4.3 \%$ & $4.7 \%$ & $4.4 \%$ & $2.1 \%$ & $2.4 \%$ & $3.7 \%$ & $0.5 \%$ & $3.2 \%$ & $2.6 \%$ & $2.2 \%$ & $1.9 \%$ \\
\hline Tashiyangtse & $4.8 \%$ & $1.9 \%$ & $1.7 \%$ & $4.1 \%$ & $1.2 \%$ & $3.8 \%$ & $4.2 \%$ & $4.3 \%$ & $3.1 \%$ & $5.8 \%$ & $1.8 \%$ & $2.5 \%$ & $3.6 \%$ & $0.5 \%$ & $3.7 \%$ & $2.9 \%$ & $2.9 \%$ & $2.2 \%$ \\
\hline Tashigang & $4.9 \%$ & $1.9 \%$ & $1.6 \%$ & $4.1 \%$ & $1.3 \%$ & $3.6 \%$ & $4.5 \%$ & $4.4 \%$ & $3.9 \%$ & $6.2 \%$ & $1.2 \%$ & $2.3 \%$ & $3.8 \%$ & $0.4 \%$ & $4.1 \%$ & $3.0 \%$ & $3.4 \%$ & $2.1 \%$ \\
\hline Thimphu & $4.9 \%$ & $1.8 \%$ & $1.7 \%$ & $3.4 \%$ & $1.4 \%$ & $4.2 \%$ & $4.4 \%$ & $4.5 \%$ & $5.6 \%$ & $5.1 \%$ & $3.0 \%$ & $3.2 \%$ & $3.7 \%$ & $0.9 \%$ & $3.1 \%$ & $2.9 \%$ & $1.6 \%$ & $1.6 \%$ \\
\hline Trongsa & $4.7 \%$ & $2.0 \%$ & $1.8 \%$ & $3.7 \%$ & $1.4 \%$ & $3.6 \%$ & $4.3 \%$ & $4.3 \%$ & $4.8 \%$ & $6.4 \%$ & $1.9 \%$ & $2.6 \%$ & $3.6 \%$ & $0.9 \%$ & $3.7 \%$ & $2.9 \%$ & $2.5 \%$ & $1.1 \%$ \\
\hline
\end{tabular}


Karma Ura, Sabina Alkire, Tshoki Zangmo \& Karma Wangdi

\begin{tabular}{|c|c|c|c|c|c|c|c|c|c|c|c|c|c|c|c|c|c|c|}
\hline Tsirang & $4.7 \%$ & $1.9 \%$ & $2.1 \%$ & $3.2 \%$ & $1.1 \%$ & $3.9 \%$ & $4.3 \%$ & $4.1 \%$ & $4.4 \%$ & $5.9 \%$ & $2.3 \%$ & $2.5 \%$ & $3.7 \%$ & $0.3 \%$ & $3.3 \%$ & $2.9 \%$ & $1.9 \%$ & $2.2 \%$ \\
\hline $\begin{array}{l}\text { Wangdue } \\
\text { Phodrang }\end{array}$ & $4.9 \%$ & $2.0 \%$ & $2.2 \%$ & $3.6 \%$ & $1.4 \%$ & $4.1 \%$ & $4.5 \%$ & $4.2 \%$ & $4.6 \%$ & $6.4 \%$ & $1.6 \%$ & $2.2 \%$ & $3.7 \%$ & $0.6 \%$ & $2.9 \%$ & $3.0 \%$ & $2.0 \%$ & $1.8 \%$ \\
\hline Zhemgang & $4.9 \%$ & $2.0 \%$ & $2.0 \%$ & $3.3 \%$ & $1.3 \%$ & $4.2 \%$ & $4.5 \%$ & $4.5 \%$ & $5.3 \%$ & $6.5 \%$ & $2.1 \%$ & $2.5 \%$ & $3.7 \%$ & $0.6 \%$ & $3.4 \%$ & $3.0 \%$ & $2.1 \%$ & $2.0 \%$ \\
\hline \multicolumn{19}{|l|}{ Region } \\
\hline Rural & $4.8 \%$ & $1.8 \%$ & $2.0 \%$ & $3.5 \%$ & $1.3 \%$ & $3.9 \%$ & $4.4 \%$ & $4.3 \%$ & $4.7 \%$ & $5.7 \%$ & $1.8 \%$ & $2.4 \%$ & $3.7 \%$ & $0.5 \%$ & $3.3 \%$ & $2.9 \%$ & $2.3 \%$ & $2.0 \%$ \\
\hline Urban & $4.9 \%$ & $1.8 \%$ & $1.8 \%$ & $3.2 \%$ & $1.4 \%$ & $4.1 \%$ & $4.5 \%$ & $4.5 \%$ & $5.3 \%$ & $5.1 \%$ & $2.9 \%$ & $3.1 \%$ & $3.7 \%$ & $0.8 \%$ & $3.0 \%$ & $2.9 \%$ & $1.4 \%$ & $1.5 \%$ \\
\hline \multicolumn{19}{|l|}{ Gender } \\
\hline Male & $4.8 \%$ & $1.8 \%$ & $1.9 \%$ & $3.5 \%$ & $1.3 \%$ & $4.0 \%$ & $4.4 \%$ & $4.4 \%$ & $5.0 \%$ & $5.4 \%$ & $2.2 \%$ & $2.9 \%$ & $3.7 \%$ & $0.7 \%$ & $3.1 \%$ & $2.9 \%$ & $2.0 \%$ & $1.8 \%$ \\
\hline Female & $4.9 \%$ & $1.8 \%$ & $1.8 \%$ & $3.2 \%$ & $1.3 \%$ & $4.0 \%$ & $4.5 \%$ & $4.4 \%$ & $4.7 \%$ & $5.7 \%$ & $2.0 \%$ & $2.3 \%$ & $3.8 \%$ & $0.4 \%$ & $3.3 \%$ & $2.9 \%$ & $1.8 \%$ & $2.0 \%$ \\
\hline \multicolumn{19}{|l|}{ Age group } \\
\hline$<=20$ & $4.8 \%$ & $1.4 \%$ & $1.6 \%$ & $2.9 \%$ & $1.3 \%$ & $4.1 \%$ & $4.5 \%$ & $4.4 \%$ & $6.2 \%$ & $6.3 \%$ & $3.2 \%$ & $3.5 \%$ & $3.5 \%$ & $0.6 \%$ & $2.5 \%$ & $2.9 \%$ & $1.4 \%$ & $1.8 \%$ \\
\hline $21-25$ & $4.8 \%$ & $1.7 \%$ & $1.6 \%$ & $2.5 \%$ & $1.4 \%$ & $4.0 \%$ & $4.6 \%$ & $4.5 \%$ & $5.4 \%$ & $6.3 \%$ & $2.8 \%$ & $3.1 \%$ & $3.7 \%$ & $0.6 \%$ & $3.5 \%$ & $2.9 \%$ & $1.6 \%$ & $1.7 \%$ \\
\hline $26-30$ & $4.9 \%$ & $1.8 \%$ & $1.8 \%$ & $2.9 \%$ & $1.3 \%$ & $4.1 \%$ & $4.5 \%$ & $4.5 \%$ & $5.1 \%$ & $5.7 \%$ & $2.9 \%$ & $3.1 \%$ & $3.7 \%$ & $0.6 \%$ & $3.0 \%$ & $2.8 \%$ & $1.7 \%$ & $1.6 \%$ \\
\hline $31-35$ & $5.0 \%$ & $1.7 \%$ & $2.0 \%$ & $3.4 \%$ & $1.4 \%$ & $4.0 \%$ & $4.5 \%$ & $4.4 \%$ & $4.7 \%$ & $5.1 \%$ & $2.5 \%$ & $2.8 \%$ & $3.7 \%$ & $0.6 \%$ & $3.3 \%$ & $3.0 \%$ & $1.9 \%$ & $1.7 \%$ \\
\hline $36-40$ & $4.9 \%$ & $1.9 \%$ & $2.0 \%$ & $3.5 \%$ & $1.3 \%$ & $3.9 \%$ & $4.3 \%$ & $4.4 \%$ & $4.5 \%$ & $5.1 \%$ & $2.3 \%$ & $2.7 \%$ & $3.7 \%$ & $0.7 \%$ & $3.3 \%$ & $2.9 \%$ & $2.0 \%$ & $1.9 \%$ \\
\hline $41-45$ & $4.8 \%$ & $1.7 \%$ & $2.0 \%$ & $3.5 \%$ & $1.3 \%$ & $4.0 \%$ & $4.4 \%$ & $4.3 \%$ & $4.4 \%$ & $5.1 \%$ & $1.6 \%$ & $2.2 \%$ & $3.7 \%$ & $0.8 \%$ & $3.0 \%$ & $2.9 \%$ & $2.2 \%$ & $2.1 \%$ \\
\hline $46-50$ & $4.9 \%$ & $1.8 \%$ & $2.0 \%$ & $3.8 \%$ & $1.2 \%$ & $4.2 \%$ & $4.4 \%$ & $4.4 \%$ & $4.0 \%$ & $5.5 \%$ & $1.4 \%$ & $2.1 \%$ & $3.8 \%$ & $0.4 \%$ & $3.5 \%$ & $2.9 \%$ & $2.1 \%$ & $2.1 \%$ \\
\hline $51-55$ & $4.9 \%$ & $1.9 \%$ & $2.1 \%$ & $4.2 \%$ & $1.3 \%$ & $4.1 \%$ & $4.3 \%$ & $4.3 \%$ & $4.9 \%$ & $5.1 \%$ & $1.4 \%$ & $2.3 \%$ & $3.7 \%$ & $0.5 \%$ & $2.7 \%$ & $3.0 \%$ & $2.7 \%$ & $2.0 \%$ \\
\hline $56-60$ & $4.7 \%$ & $1.9 \%$ & $2.0 \%$ & $4.2 \%$ & $1.2 \%$ & $3.8 \%$ & $4.0 \%$ & $4.3 \%$ & $4.8 \%$ & $5.5 \%$ & $1.3 \%$ & $2.2 \%$ & $3.8 \%$ & $0.3 \%$ & $3.2 \%$ & $2.9 \%$ & $2.5 \%$ & $1.9 \%$ \\
\hline $61-65$ & $4.7 \%$ & $2.1 \%$ & $2.1 \%$ & $4.3 \%$ & $1.2 \%$ & $3.6 \%$ & $4.1 \%$ & $4.1 \%$ & $5.0 \%$ & $5.8 \%$ & $0.5 \%$ & $1.8 \%$ & $3.8 \%$ & $0.4 \%$ & $3.3 \%$ & $3.0 \%$ & $2.8 \%$ & $2.1 \%$ \\
\hline$>65$ & $4.7 \%$ & $2.1 \%$ & $2.2 \%$ & $4.6 \%$ & $1.0 \%$ & $4.0 \%$ & $4.3 \%$ & $4.0 \%$ & $5.9 \%$ & $5.6 \%$ & $0.4 \%$ & $1.5 \%$ & $3.8 \%$ & $0.3 \%$ & $3.0 \%$ & $3.1 \%$ & $2.3 \%$ & $2.5 \%$ \\
\hline
\end{tabular}


An Extensive Analysis of GNH Index

Table II-B (cont.) Understanding Happiness - Composition \& Contributions The table shows the percentage contribution to overall happiness

Suggested citation: Ura, Karma; Alkire, Sabina; and Zangmo, Tshoki (Nov 2011) www.grossnationalhappiness.com ophi.qeh.ox.ac.uk

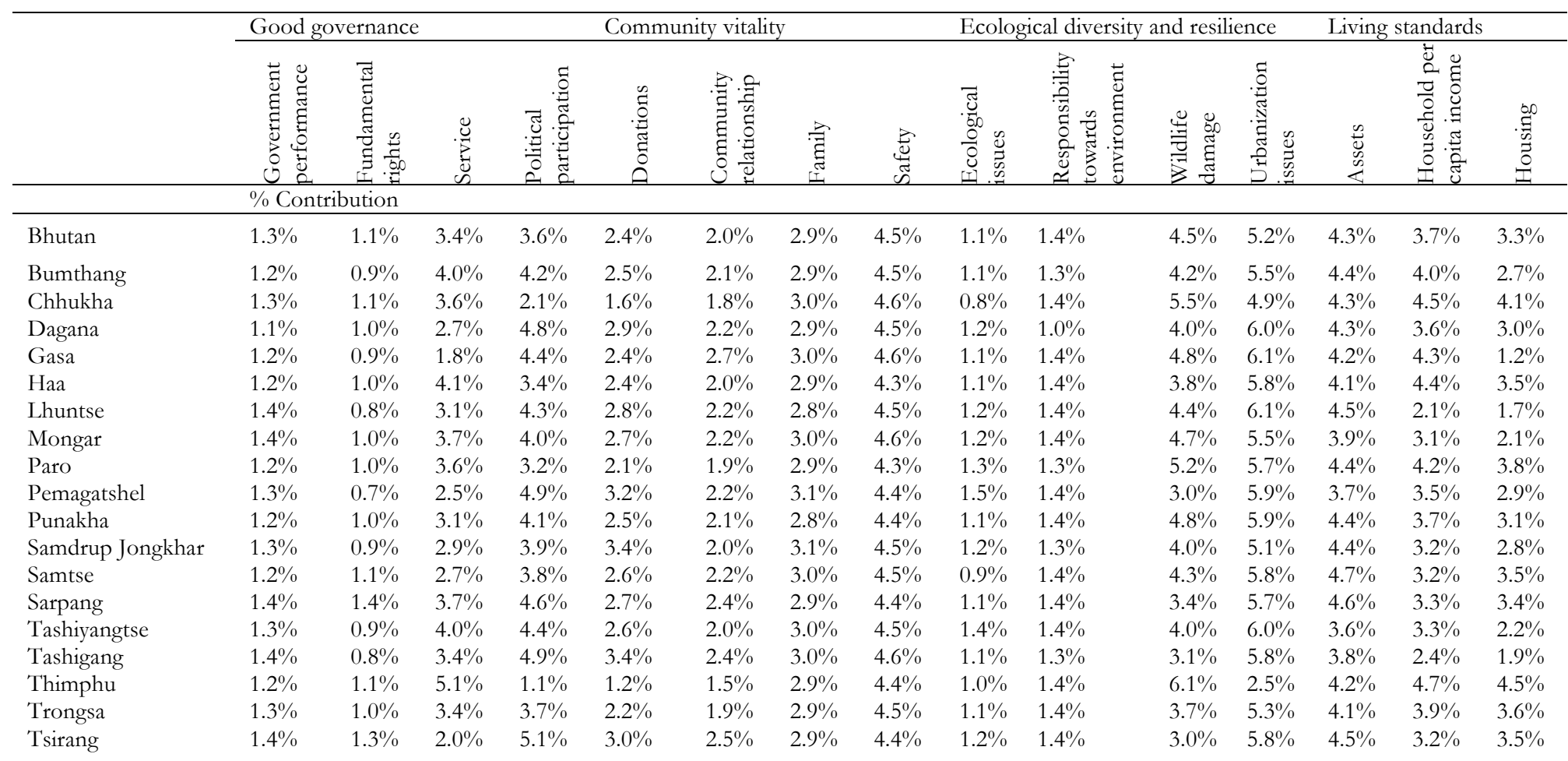


Karma Ura, Sabina Alkire, Tshoki Zangmo \& Karma Wangdi

\begin{tabular}{|c|c|c|c|c|c|c|c|c|c|c|c|c|c|c|c|}
\hline Wangdue Phodrang & $1.4 \%$ & $1.1 \%$ & $2.7 \%$ & $4.6 \%$ & $2.9 \%$ & $2.0 \%$ & $2.9 \%$ & $4.5 \%$ & $0.9 \%$ & $1.3 \%$ & $4.0 \%$ & $4.9 \%$ & $4.4 \%$ & $3.7 \%$ & $2.6 \%$ \\
\hline \multicolumn{16}{|l|}{ Region } \\
\hline Rural & $1.3 \%$ & $1.1 \%$ & $2.7 \%$ & $4.7 \%$ & $2.9 \%$ & $2.4 \%$ & $2.9 \%$ & $4.5 \%$ & $1.1 \%$ & $1.4 \%$ & $3.7 \%$ & $6.0 \%$ & $4.2 \%$ & $3.1 \%$ & $2.7 \%$ \\
\hline Urban & $1.2 \%$ & $1.0 \%$ & $5.0 \%$ & $1.2 \%$ & $1.3 \%$ & $1.4 \%$ & $3.0 \%$ & $4.4 \%$ & $1.0 \%$ & $1.4 \%$ & $6.1 \%$ & $3.6 \%$ & $4.5 \%$ & $4.8 \%$ & $4.6 \%$ \\
\hline Male & $1.2 \%$ & $1.1 \%$ & $3.1 \%$ & $4.0 \%$ & $2.7 \%$ & $2.2 \%$ & $2.9 \%$ & $4.4 \%$ & $1.1 \%$ & $1.4 \%$ & $4.1 \%$ & $5.2 \%$ & $4.2 \%$ & $3.4 \%$ & $3.0 \%$ \\
\hline Female & $1.3 \%$ & $1.0 \%$ & $4.0 \%$ & $2.9 \%$ & $2.0 \%$ & $1.9 \%$ & $2.9 \%$ & $4.5 \%$ & $1.1 \%$ & $1.3 \%$ & $5.1 \%$ & $5.1 \%$ & $4.4 \%$ & $4.1 \%$ & $3.7 \%$ \\
\hline \multicolumn{16}{|l|}{ Age group } \\
\hline $31-35$ & $1.2 \%$ & $1.0 \%$ & $3.3 \%$ & $3.6 \%$ & $2.2 \%$ & $2.0 \%$ & $3.0 \%$ & $4.5 \%$ & $1.1 \%$ & $1.4 \%$ & $4.7 \%$ & $5.2 \%$ & $4.3 \%$ & $3.7 \%$ & $3.4 \%$ \\
\hline $36-40$ & $1.2 \%$ & $1.0 \%$ & $3.4 \%$ & $3.6 \%$ & $2.5 \%$ & $2.0 \%$ & $3.0 \%$ & $4.5 \%$ & $1.0 \%$ & $1.4 \%$ & $4.5 \%$ & $4.8 \%$ & $4.4 \%$ & $3.9 \%$ & $3.5 \%$ \\
\hline $41-45$ & $1.2 \%$ & $1.2 \%$ & $3.7 \%$ & $4.1 \%$ & $2.9 \%$ & $2.4 \%$ & $2.9 \%$ & $4.5 \%$ & $1.0 \%$ & $1.4 \%$ & $4.3 \%$ & $5.1 \%$ & $4.4 \%$ & $3.4 \%$ & $3.1 \%$ \\
\hline $46-50$ & $1.3 \%$ & $1.1 \%$ & $3.2 \%$ & $4.3 \%$ & $2.8 \%$ & $2.4 \%$ & $3.0 \%$ & $4.5 \%$ & $1.0 \%$ & $1.4 \%$ & $3.8 \%$ & $5.5 \%$ & $4.6 \%$ & $3.2 \%$ & $3.1 \%$ \\
\hline $51-55$ & $1.3 \%$ & $1.0 \%$ & $3.0 \%$ & $4.8 \%$ & $2.9 \%$ & $2.4 \%$ & $2.9 \%$ & $4.6 \%$ & $1.2 \%$ & $1.3 \%$ & $3.4 \%$ & $5.7 \%$ & $4.0 \%$ & $3.4 \%$ & $2.7 \%$ \\
\hline $56-60$ & $1.4 \%$ & $1.1 \%$ & $3.3 \%$ & $5.2 \%$ & $2.7 \%$ & $2.4 \%$ & $3.0 \%$ & $4.5 \%$ & $1.1 \%$ & $1.4 \%$ & $3.6 \%$ & $5.6 \%$ & $4.2 \%$ & $3.4 \%$ & $2.8 \%$ \\
\hline
\end{tabular}


An Extensive Analysis of GNH Index

Table III Understanding Happiness - Censored headcounts The proportion of people who are happy and experience sufficiency in each indicator.

Suggested citation: Ura, Karma; Alkire, Sabina; and Zangmo, Tshoki (Nov 2011) www.grossnationalhappiness.com ophi.qeh.ox.ac.uk

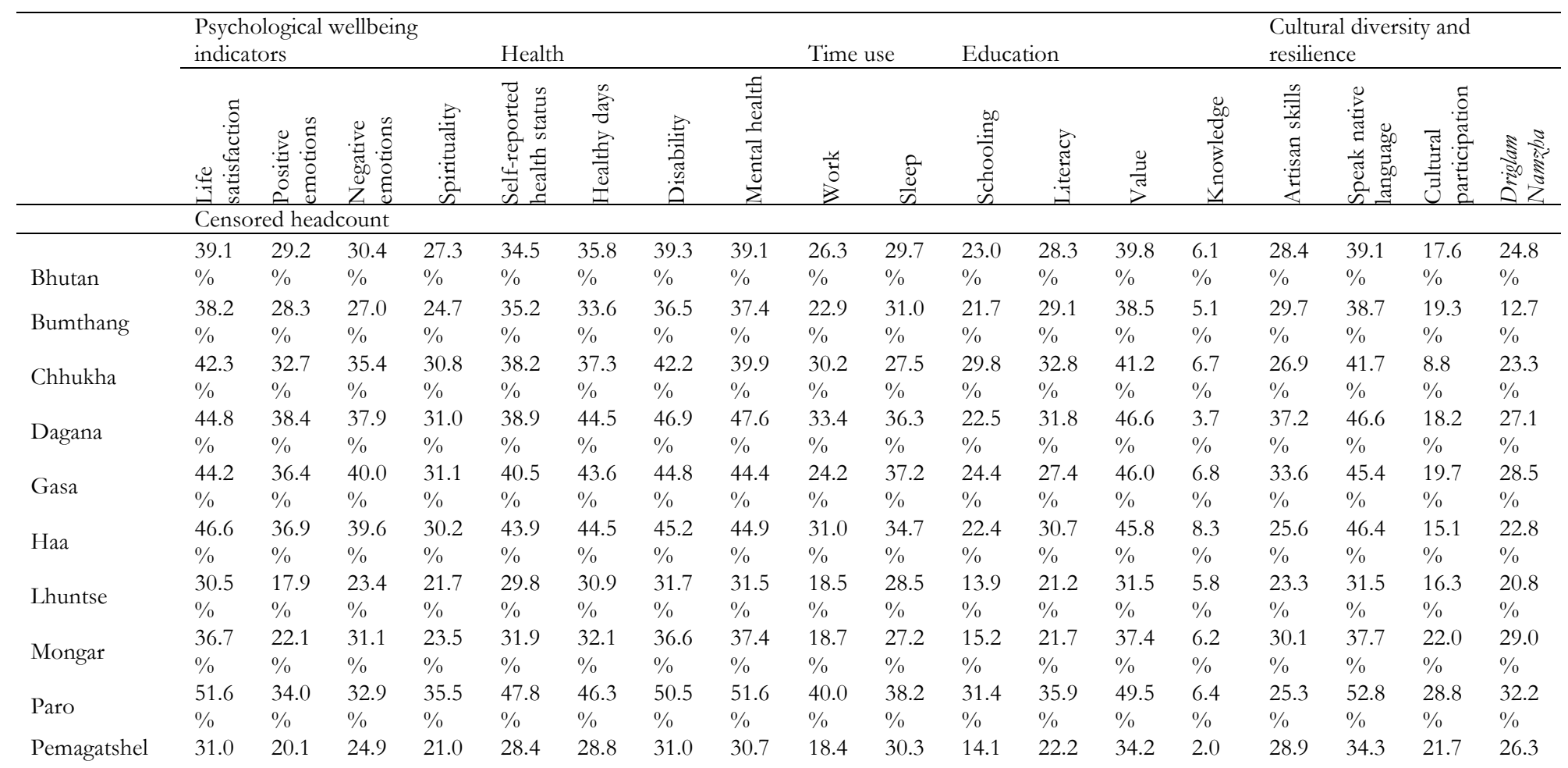


Karma Ura, Sabina Alkire, Tshoki Zangmo \& Karma Wangdi

\begin{tabular}{|c|c|c|c|c|c|c|c|c|c|c|c|c|c|c|c|c|c|c|}
\hline Punakha & $\%$ & $\%$ & $\%$ & $\%$ & $\%$ & $\%$ & $\%$ & $\%$ & $\%$ & $\%$ & $\%$ & $\%$ & $\%$ & $\%$ & $\%$ & $\%$ & $\%$ & $\%$ \\
\hline Samdrup & 20.6 & 14.5 & 13.7 & 15.4 & 19.7 & 19.0 & 22.2 & 21.8 & 15.5 & 19.7 & 12.1 & 17.3 & 23.4 & 3.1 & 17.8 & 22.2 & 13.4 & 14.0 \\
\hline Jongkhar & $\%$ & $\%$ & $\%$ & $\%$ & $\%$ & $\%$ & $\%$ & $\%$ & $\%$ & $\%$ & $\%$ & $\%$ & $\%$ & $\%$ & $\%$ & $\%$ & $\%$ & $\%$ \\
\hline Samtse & $\%$ & $\%$ & $\%$ & $\%$ & $\%$ & $\%$ & $\%$ & $\%$ & $\%$ & $\%$ & $\%$ & $\%$ & $\%$ & $\%$ & $\%$ & $\%$ & $\%$ & $\%$ \\
\hline \multirow{2}{*}{ Sarpang } & 48.9 & 42.6 & 39.5 & 34.2 & 33.6 & 46.9 & 48.8 & 48.9 & 32.5 & 29.9 & 29.2 & 32.3 & 50.9 & 6.4 & 36.9 & 45.4 & 25.4 & 32.2 \\
\hline & $\%$ & $\%$ & $\%$ & $\%$ & $\%$ & $\%$ & $\%$ & $\%$ & $\%$ & $\%$ & $\%$ & $\%$ & $\%$ & $\%$ & $\%$ & $\%$ & $\%$ & $\%$ \\
\hline \multirow{2}{*}{ Tashigang } & 31.6 & 23.8 & 20.7 & 26.3 & 27.5 & 25.9 & 31.9 & 31.4 & 16.8 & 26.7 & 10.1 & 19.7 & 32.6 & 3.7 & 29.3 & 31.8 & 24.2 & 22.7 \\
\hline & $\%$ & $\%$ & $\%$ & $\%$ & $\%$ & $\%$ & $\%$ & $\%$ & $\%$ & $\%$ & $\%$ & $\%$ & $\%$ & $\%$ & $\%$ & $\%$ & $\%$ & $\%$ \\
\hline \multirow{2}{*}{ Thimphu } & 44.5 & 32.5 & 30.9 & 30.6 & 42.6 & 42.7 & 44.6 & 45.7 & 34.0 & 31.1 & 36.0 & 38.2 & 45.2 & 10.5 & 31.4 & 43.3 & 16.3 & 24.4 \\
\hline & $\%$ & $\%$ & $\%$ & $\%$ & $\%$ & $\%$ & $\%$ & $\%$ & $\%$ & $\%$ & $\%$ & $\%$ & $\%$ & $\%$ & $\%$ & $\%$ & $\%$ & $\%$ \\
\hline \multirow{2}{*}{ Trongsa } & 26.7 & 22.8 & 20.5 & 21.0 & 25.9 & 22.8 & 27.5 & 27.3 & 18.1 & 24.2 & 14.8 & 19.6 & 27.6 & 6.5 & 23.6 & 27.3 & 15.7 & 10.7 \\
\hline & $\%$ & $\%$ & $\%$ & $\%$ & $\%$ & $\%$ & $\%$ & $\%$ & $\%$ & $\%$ & $\%$ & $\%$ & $\%$ & $\%$ & $\%$ & $\%$ & $\%$ & $\%$ \\
\hline Tsirang & 45.1 & 35.4 & 39.2 & 30.1 & 35.4 & 41.4 & 45.9 & 43.5 & 27.6 & 37.2 & 29.6 & 31.7 & 47.3 & 4.1 & 34.8 & 46.3 & 20.4 & 35.0 \\
\hline \multicolumn{19}{|l|}{ Region } \\
\hline & 35.7 & 26.8 & 28.9 & 25.7 & 30.9 & 32.3 & 35.8 & 35.5 & 23.2 & 28.2 & 17.4 & 23.8 & 36.6 & 4.5 & 26.8 & 35.9 & 18.6 & 25.1 \\
\hline \multirow[t]{2}{*}{ Rural } & $\%$ & $\%$ & $\%$ & $\%$ & $\%$ & $\%$ & $\%$ & $\%$ & $\%$ & $\%$ & $\%$ & $\%$ & $\%$ & $\%$ & $\%$ & $\%$ & $\%$ & $\%$ \\
\hline & 48.2 & 35.5 & 34.7 & 31.6 & 44.3 & 45.0 & 48.9 & 48.9 & 34.6 & 33.6 & 37.9 & 40.5 & 48.3 & 10.3 & 32.7 & 47.6 & 14.9 & 23.9 \\
\hline \multirow{2}{*}{$\begin{array}{l}\text { Urban } \\
\text { Gender }\end{array}$} & $\%$ & $\%$ & $\%$ & $\%$ & $\%$ & $\%$ & $\%$ & $\%$ & $\%$ & $\%$ & $\%$ & $\%$ & $\%$ & $\%$ & $\%$ & $\%$ & $\%$ & $\%$ \\
\hline & 46.6 & 35.5 & 37.3 & 34.0 & 41.2 & 42.6 & 46.6 & 46.7 & 32.1 & 34.6 & 28.7 & 36.8 & 47.2 & 8.9 & 33.1 & 46.9 & 21.8 & 28.4 \\
\hline Male & $\%$ & $\%$ & $\%$ & $\%$ & $\%$ & $\%$ & $\%$ & $\%$ & $\%$ & $\%$ & $\%$ & $\%$ & $\%$ & $\%$ & $\%$ & $\%$ & $\%$ & $\%$ \\
\hline
\end{tabular}

184 
An Extensive Analysis of GNH Index

\begin{tabular}{|c|c|c|c|c|c|c|c|c|c|c|c|c|c|c|c|c|c|c|}
\hline & 31.6 & 22.8 & 23.6 & 20.6 & 27.8 & 28.9 & 32.1 & 31.5 & 20.5 & 24.8 & 17.2 & 19.8 & 32.4 & 3.2 & 23.7 & 31.3 & 13.3 & 21.1 \\
\hline \multirow{2}{*}{$\begin{array}{l}\text { Female } \\
\text { Age group }\end{array}$} & $\%$ & $\%$ & $\%$ & $\%$ & $\%$ & $\%$ & $\%$ & $\%$ & $\%$ & $\%$ & $\%$ & $\%$ & $\%$ & $\%$ & $\%$ & $\%$ & $\%$ & $\%$ \\
\hline & 40.4 & 23.3 & 25.9 & 23.9 & 36.6 & 37.6 & 42.0 & 40.8 & 34.7 & 35.2 & 35.5 & 38.5 & 39.4 & 6.6 & 23.1 & 40.1 & 12.7 & 24.4 \\
\hline \multirow[t]{2}{*}{$<=20$} & $\%$ & $\%$ & $\%$ & $\%$ & $\%$ & $\%$ & $\%$ & $\%$ & $\%$ & $\%$ & $\%$ & $\%$ & $\%$ & $\%$ & $\%$ & $\%$ & $\%$ & $\%$ \\
\hline & 47.2 & 34.0 & 31.7 & 24.5 & 43.9 & 43.0 & 49.4 & 48.4 & 35.3 & 40.9 & 36.4 & 40.2 & 48.4 & 8.0 & 38.4 & 47.1 & 16.9 & 27.0 \\
\hline \multirow[t]{2}{*}{$21-25$} & $\%$ & $\%$ & $\%$ & $\%$ & $\%$ & $\%$ & $\%$ & $\%$ & $\%$ & $\%$ & $\%$ & $\%$ & $\%$ & $\%$ & $\%$ & $\%$ & $\%$ & $\%$ \\
\hline & 45.7 & 33.8 & 32.7 & 27.2 & 40.6 & 42.1 & 46.3 & 46.3 & 31.6 & 35.6 & 35.7 & 38.2 & 45.4 & 7.7 & 31.4 & 44.0 & 17.7 & 24.2 \\
\hline \multirow[t]{2}{*}{$26-30$} & $\%$ & $\%$ & $\%$ & $\%$ & $\%$ & $\%$ & $\%$ & $\%$ & $\%$ & $\%$ & $\%$ & $\%$ & $\%$ & $\%$ & $\%$ & $\%$ & $\%$ & $\%$ \\
\hline & 41.2 & 28.9 & 33.2 & 27.9 & 38.0 & 36.6 & 41.2 & 40.3 & 26.1 & 28.1 & 27.5 & 31.3 & 41.2 & 6.5 & 30.6 & 41.3 & 17.4 & 23.3 \\
\hline \multirow[t]{2}{*}{$31-35$} & $\%$ & $\%$ & $\%$ & $\%$ & $\%$ & $\%$ & $\%$ & $\%$ & $\%$ & $\%$ & $\%$ & $\%$ & $\%$ & $\%$ & $\%$ & $\%$ & $\%$ & $\%$ \\
\hline & 37.2 & 29.1 & 29.8 & 26.7 & 33.6 & 33.0 & 36.3 & 37.2 & 22.5 & 25.9 & 22.9 & 27.0 & 37.2 & 7.0 & 27.6 & 36.2 & 16.7 & 23.7 \\
\hline \multirow[t]{2}{*}{$36-40$} & $\%$ & $\%$ & $\%$ & $\%$ & $\%$ & $\%$ & $\%$ & $\%$ & $\%$ & $\%$ & $\%$ & $\%$ & $\%$ & $\%$ & $\%$ & $\%$ & $\%$ & $\%$ \\
\hline & 37.2 & 27.0 & 31.3 & 27.1 & 33.4 & 34.6 & 38.1 & 37.2 & 22.8 & 26.4 & 16.6 & 23.1 & 38.5 & 8.2 & 26.2 & 37.9 & 19.0 & 27.0 \\
\hline \multirow[t]{2}{*}{$41-45$} & $\%$ & $\%$ & $\%$ & $\%$ & $\%$ & $\%$ & $\%$ & $\%$ & $\%$ & $\%$ & $\%$ & $\%$ & $\%$ & $\%$ & $\%$ & $\%$ & $\%$ & $\%$ \\
\hline & 39.3 & 29.3 & 32.5 & 30.2 & 33.0 & 37.2 & 39.5 & 39.6 & 21.3 & 29.2 & 15.2 & 22.3 & 40.3 & 4.3 & 31.5 & 39.4 & 18.6 & 28.5 \\
\hline \multirow[t]{2}{*}{$46-50$} & $\%$ & $\%$ & $\%$ & $\%$ & $\%$ & $\%$ & $\%$ & $\%$ & $\%$ & $\%$ & $\%$ & $\%$ & $\%$ & $\%$ & $\%$ & $\%$ & $\%$ & $\%$ \\
\hline & 32.9 & 25.5 & 28.7 & 28.2 & 28.7 & 30.4 & 32.0 & 32.0 & 21.8 & 22.7 & 12.9 & 20.9 & 33.4 & 4.8 & 20.4 & 33.4 & 20.0 & 22.2 \\
\hline \multirow[t]{2}{*}{ 51-55 } & $\%$ & $\%$ & $\%$ & $\%$ & $\%$ & $\%$ & $\%$ & $\%$ & $\%$ & $\%$ & $\%$ & $\%$ & $\%$ & $\%$ & $\%$ & $\%$ & $\%$ & $\%$ \\
\hline & 35.1 & 27.8 & 29.1 & 31.5 & 29.5 & 31.5 & 33.2 & 35.5 & 23.9 & 27.3 & 13.3 & 21.5 & 37.6 & 2.7 & 26.7 & 35.7 & 20.3 & 23.8 \\
\hline \multirow{2}{*}{$56-60$} & $\%$ & $\%$ & $\%$ & $\%$ & $\%$ & $\%$ & $\%$ & $\%$ & $\%$ & $\%$ & $\%$ & $\%$ & $\%$ & $\%$ & $\%$ & $\%$ & $\%$ & $\%$ \\
\hline & 30.1 & 26.7 & 27.1 & 27.6 & 24.7 & 25.4 & 29.3 & 28.9 & 21.0 & 24.7 & 4.1 & 15.1 & 32.1 & 3.1 & 23.1 & 32.0 & 19.7 & 22.7 \\
\hline \multirow[t]{2}{*}{ 61-65 } & $\%$ & $\%$ & $\%$ & $\%$ & $\%$ & $\%$ & $\%$ & $\%$ & $\%$ & $\%$ & $\%$ & $\%$ & $\%$ & $\%$ & $\%$ & $\%$ & $\%$ & $\%$ \\
\hline & 26.0 & 23.7 & 24.1 & 25.5 & 18.9 & 25.2 & 26.5 & 24.8 & 21.8 & 20.8 & 3.0 & 11.3 & 28.3 & 2.0 & 18.4 & 28.7 & 14.4 & 23.2 \\
\hline$>65$ & $\%$ & $\%$ & $\%$ & $\%$ & $\%$ & $\%$ & $\%$ & $\%$ & $\%$ & $\%$ & $\%$ & $\%$ & $\%$ & $\%$ & $\%$ & $\%$ & $\%$ & $\%$ \\
\hline
\end{tabular}


Karma Ura, Sabina Alkire, Tshoki Zangmo \& Karma Wangdi

Table III (cont.) Understanding Happiness - Censored headcounts The proportion of people who are happy and experience sufficiency in each indicator.

Suggested citation: Ura, Karma; Alkire, Sabina; and Zangmo, Tshoki (Nov 2011) www.grossnationalhappiness.com ophi.qeh.ox.ac.uk

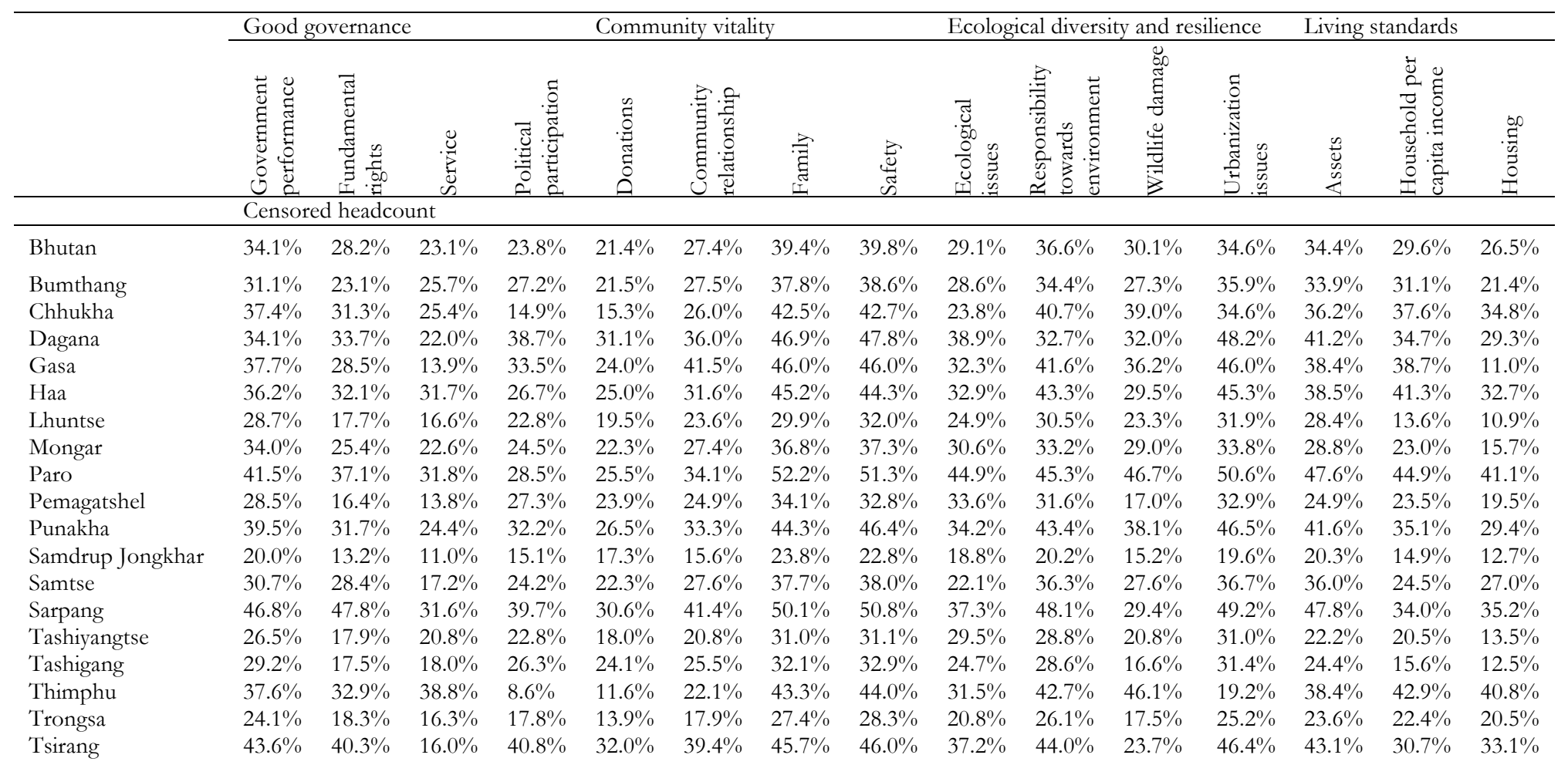

186 
An Extensive Analysis of GNH Index

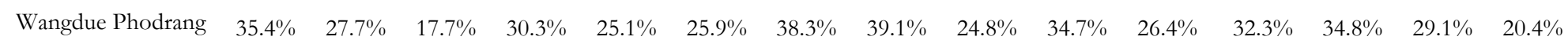

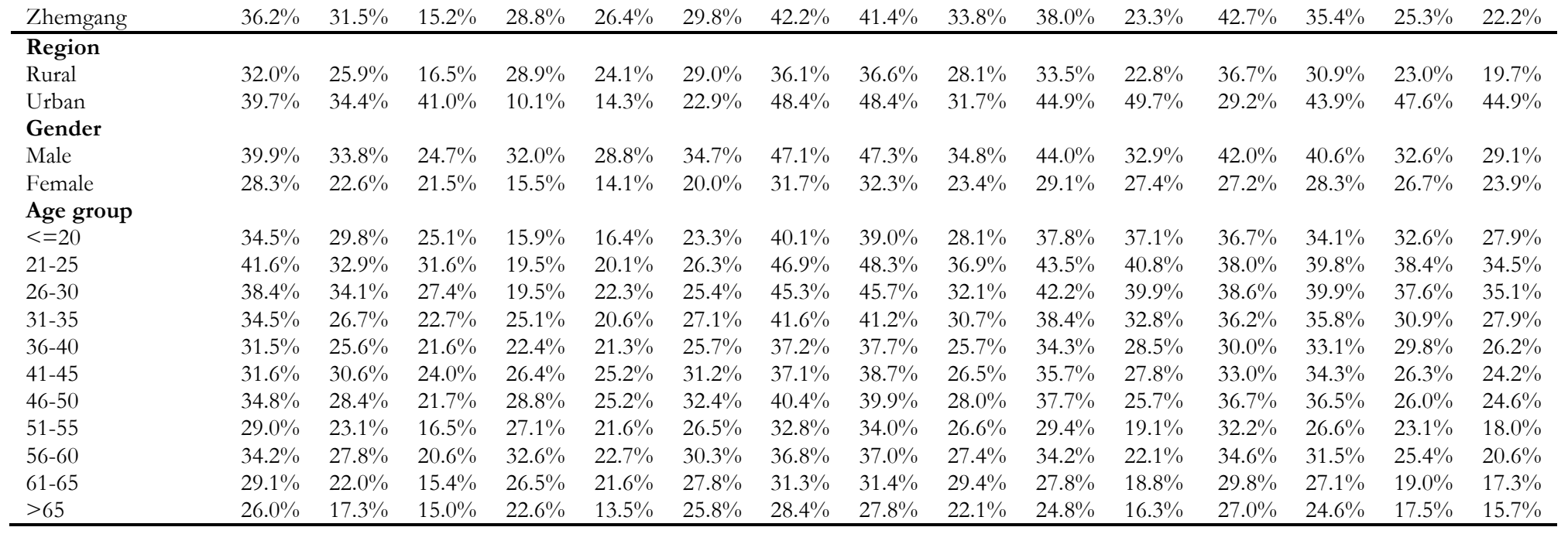


Karma Ura, Sabina Alkire, Tshoki Zangmo \& Karma Wangdi

Table IV Understanding Happiness - Raw headcounts The table shows the proportion of people who experience sufficiency in each of the indicators.

Suggested citation: Ura, Karma; Alkire, Sabina; and Zangmo, Tshoki (Nov 2011) www.grossnationalhappiness.com ophi.qeh.ox.ac.uk

\begin{tabular}{|c|c|c|c|c|c|c|c|c|c|c|c|c|c|c|c|c|c|c|}
\hline & \multicolumn{4}{|c|}{ Psychological wellbeing } & \multicolumn{4}{|c|}{ Health } & \multicolumn{2}{|c|}{ Time use } & \multicolumn{3}{|c|}{ Education } & \multicolumn{5}{|c|}{$\begin{array}{l}\text { Cultural diversity and } \\
\text { resilience }\end{array}$} \\
\hline & 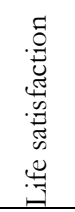 & 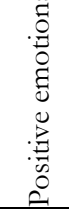 & 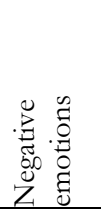 & 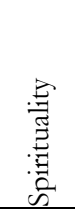 & 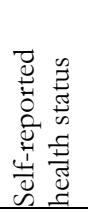 & 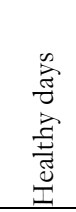 & 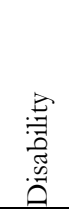 & 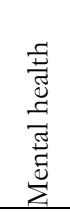 & $\frac{4}{3}$ & $\frac{\vec{y}}{\frac{\ddot{n}}{n}}$ & 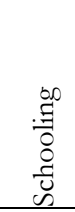 & 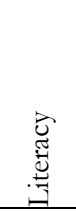 & 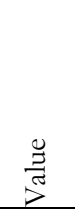 & 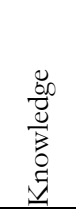 & 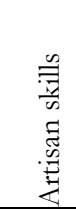 & 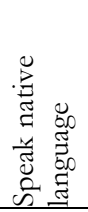 & 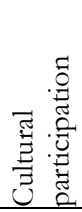 & 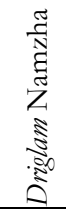 \\
\hline & \multicolumn{18}{|c|}{ Raw headcount } \\
\hline Bumthang & $\begin{array}{l}75.2 \\
\%\end{array}$ & $\begin{array}{l}58.8 \\
\%\end{array}$ & $\begin{array}{l}54.4 \\
\%\end{array}$ & $\begin{array}{l}52.6 \\
\%\end{array}$ & $\begin{array}{l}73.5 \\
\%\end{array}$ & $\begin{array}{l}68.7 \\
\%\end{array}$ & $\begin{array}{l}88.4 \\
\%\end{array}$ & $\begin{array}{l}80.2 \\
\%\end{array}$ & $\begin{array}{l}43.4 \\
\%\end{array}$ & $\begin{array}{l}67.0 \\
\%\end{array}$ & $\begin{array}{l}34.9 \\
\%\end{array}$ & $\begin{array}{l}48.5 \\
\%\end{array}$ & $\begin{array}{l}95.5 \\
\%\end{array}$ & $\begin{array}{l}7.2 \\
\%\end{array}$ & $\begin{array}{l}70.4 \\
\%\end{array}$ & $\begin{array}{l}96.7 \\
\%\end{array}$ & $\begin{array}{l}37.6 \\
\%\end{array}$ & $\begin{array}{l}33.4 \\
\%\end{array}$ \\
\hline Chhukha & $\begin{array}{l}86.9 \\
\%\end{array}$ & $\begin{array}{l}58.8 \\
\%\end{array}$ & $\begin{array}{l}72.2 \\
\%\end{array}$ & $\begin{array}{l}52.0 \\
\%\end{array}$ & $\begin{array}{l}72.3 \\
\%\end{array}$ & $\begin{array}{l}79.4 \\
\%\end{array}$ & $\begin{array}{l}91.8 \\
\%\end{array}$ & $\begin{array}{l}83.2 \\
\%\end{array}$ & $\begin{array}{l}52.1 \\
\%\end{array}$ & $\begin{array}{l}54.2 \\
\%\end{array}$ & $\begin{array}{l}49.8 \\
\%\end{array}$ & $\begin{array}{l}55.6 \\
\%\end{array}$ & $\begin{array}{l}96.9 \\
\%\end{array}$ & $\begin{array}{l}7.4 \\
\%\end{array}$ & $\begin{array}{l}48.3 \\
\%\end{array}$ & $\begin{array}{l}96.0 \\
\%\end{array}$ & $\begin{array}{l}17.6 \\
\%\end{array}$ & $\begin{array}{l}56.6 \\
\%\end{array}$ \\
\hline Dagana & $\begin{array}{l}82.7 \\
\%\end{array}$ & $\begin{array}{l}76.8 \\
\%\end{array}$ & $\begin{array}{l}74.1 \\
\%\end{array}$ & $\begin{array}{l}56.5 \\
\%\end{array}$ & $\begin{array}{l}76.2 \\
\%\end{array}$ & $\begin{array}{l}83.5 \\
\%\end{array}$ & $\begin{array}{l}91.9 \\
\%\end{array}$ & $\begin{array}{l}93.8 \\
\%\end{array}$ & $\begin{array}{l}51.9 \\
\%\end{array}$ & $\begin{array}{l}69.8 \\
\%\end{array}$ & $\begin{array}{l}30.8 \\
\%\end{array}$ & $\begin{array}{l}44.8 \\
\%\end{array}$ & $\begin{array}{l}95.8 \\
\%\end{array}$ & $\begin{array}{l}4.0 \\
\%\end{array}$ & $\begin{array}{l}61.4 \\
\%\end{array}$ & $\begin{array}{l}94.6 \\
\%\end{array}$ & $\begin{array}{l}31.0 \\
\%\end{array}$ & $\begin{array}{l}53.3 \\
\%\end{array}$ \\
\hline Lhuntse & $\begin{array}{l}76.8 \\
\%\end{array}$ & $\begin{array}{l}44.7 \\
\%\end{array}$ & $\begin{array}{l}56.0 \\
\%\end{array}$ & $\begin{array}{l}60.4 \\
\%\end{array}$ & $\begin{array}{l}70.0 \\
\%\end{array}$ & $\begin{array}{l}72.3 \\
\%\end{array}$ & $\begin{array}{l}83.4 \\
\%\end{array}$ & $\begin{array}{l}82.4 \\
\%\end{array}$ & $\begin{array}{l}37.8 \\
\%\end{array}$ & $\begin{array}{l}83.7 \\
\%\end{array}$ & $\begin{array}{l}21.1 \\
\%\end{array}$ & $\begin{array}{l}38.0 \\
\%\end{array}$ & $\begin{array}{l}97.5 \\
\%\end{array}$ & $\begin{array}{l}10.4 \\
\%\end{array}$ & $\begin{array}{l}72.6 \\
\%\end{array}$ & $\begin{array}{l}98.5 \\
\%\end{array}$ & $\begin{array}{l}41.2 \\
\%\end{array}$ & $\begin{array}{l}69.0 \\
\%\end{array}$ \\
\hline Mongar & $\begin{array}{l}84.0 \\
\%\end{array}$ & $\begin{array}{l}44.7 \\
\%\end{array}$ & $\begin{array}{l}72.4 \\
\%\end{array}$ & $\begin{array}{l}50.9 \\
\%\end{array}$ & $\begin{array}{l}78.2 \\
\%\end{array}$ & $\begin{array}{l}76.7 \\
\%\end{array}$ & $\begin{array}{l}89.9 \\
\%\end{array}$ & $\begin{array}{l}89.5 \\
\%\end{array}$ & $\begin{array}{l}29.3 \\
\%\end{array}$ & $\begin{array}{l}66.8 \\
\%\end{array}$ & $\begin{array}{l}26.7 \\
\%\end{array}$ & $\begin{array}{l}38.4 \\
\%\end{array}$ & $\begin{array}{l}99.2 \\
\%\end{array}$ & $\begin{array}{l}7.6 \\
\%\end{array}$ & $\begin{array}{l}75.0 \\
\%\end{array}$ & $\begin{array}{l}99.1 \\
\%\end{array}$ & $\begin{array}{l}44.3 \\
\%\end{array}$ & $\begin{array}{l}77.9 \\
\%\end{array}$ \\
\hline Paro & 85.3 & 55.3 & 54.3 & 57.1 & 79.6 & 78.5 & 86.5 & 90.0 & 60.0 & 65.3 & 44.1 & 51.8 & 95.3 & 7.9 & 45.2 & 97.3 & 39.8 & 61.1 \\
\hline
\end{tabular}

188 
An Extensive Analysis of GNH Index

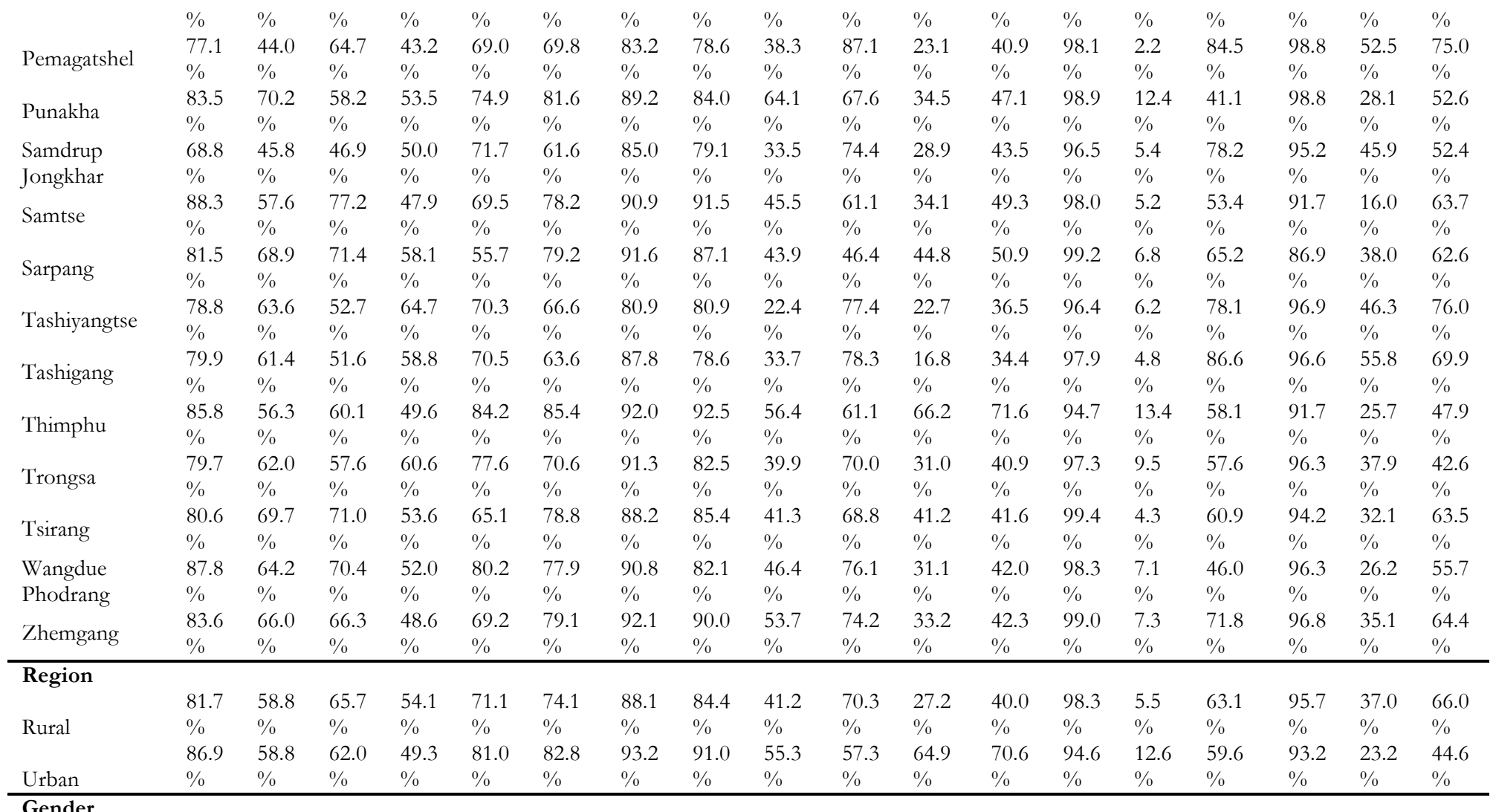


Karma Ura, Sabina Alkire, Tshoki Zangmo \& Karma Wangdi

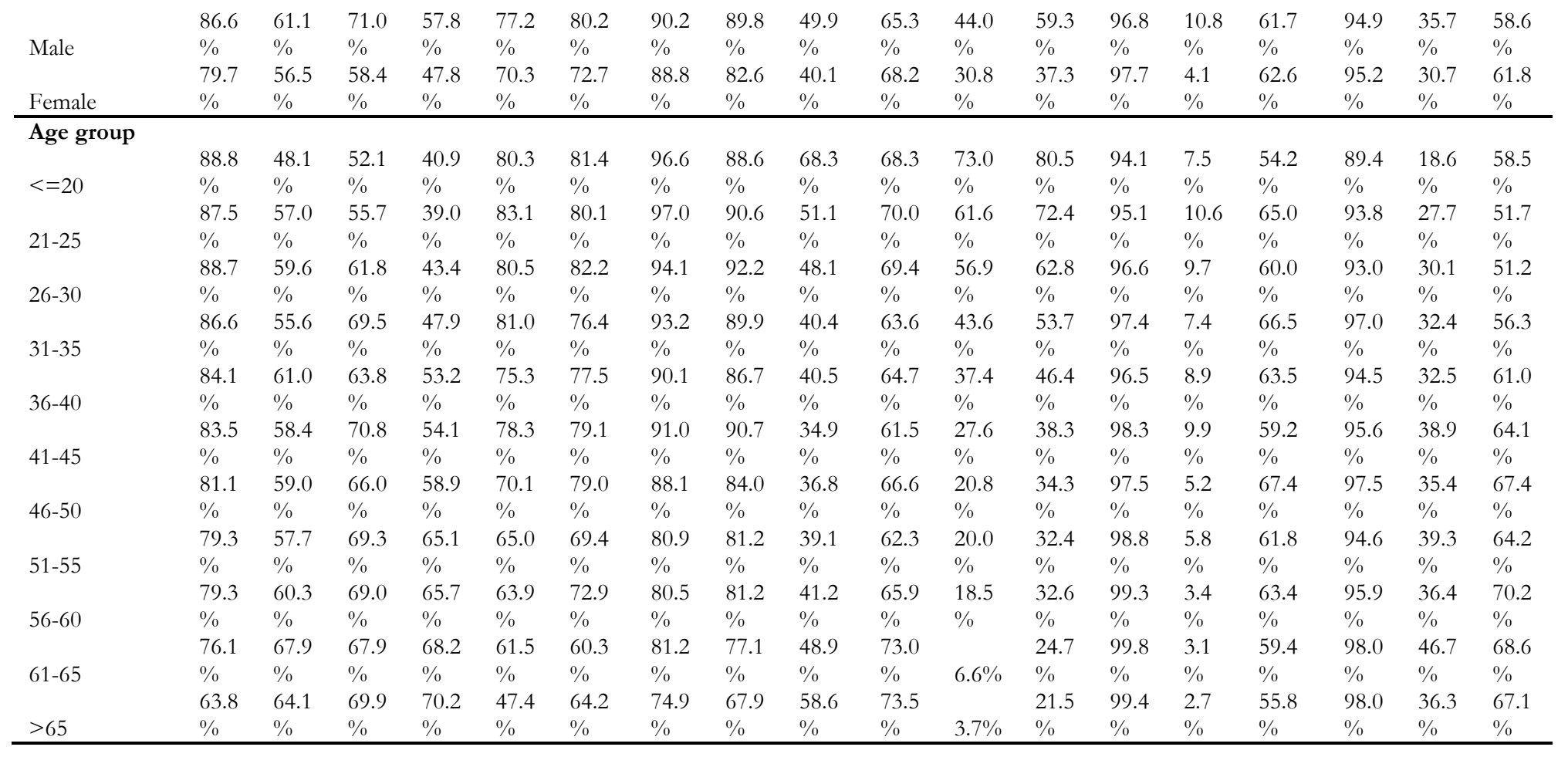


An Extensive Analysis of GNH Index

Table IV (cont.) Understanding Happiness - Raw headcounts The table shows the proportion of people who experience sufficiency in each indicator.

Suggested citation: Ura, Karma; Alkire, Sabina; and Zangmo, Tshoki (Nov 2011) www.grossnationalhappiness.com ophi.qeh.ox.ac.uk

\begin{tabular}{|c|c|c|c|c|c|c|c|c|c|c|c|c|c|c|c|}
\hline & \multicolumn{3}{|c|}{ Good governance } & \multicolumn{5}{|c|}{ Community vitality } & \multicolumn{4}{|c|}{ Ecological diversity and resilience } & \multicolumn{3}{|c|}{ Living standards } \\
\hline & 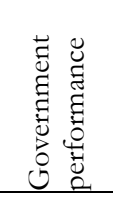 & 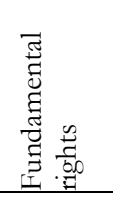 & 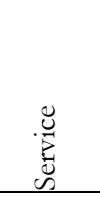 & 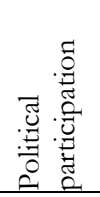 & 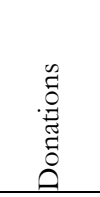 & 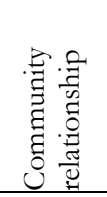 & 莞 & 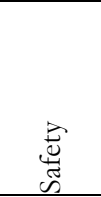 & 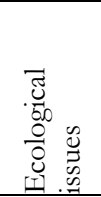 & 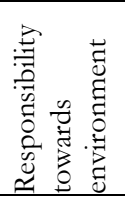 & 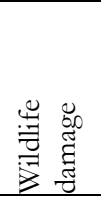 & 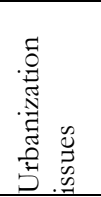 & 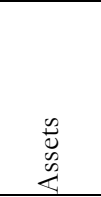 & 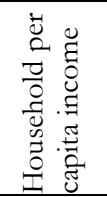 & 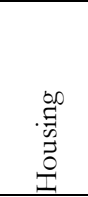 \\
\hline & \multicolumn{15}{|c|}{ Raw headcount } \\
\hline Bhutan & $79.5 \%$ & $62.2 \%$ & $40.9 \%$ & $56.5 \%$ & $46.3 \%$ & $62.5 \%$ & $92.9 \%$ & $96.2 \%$ & $69.2 \%$ & $84.3 \%$ & $57.3 \%$ & $84.8 \%$ & $74.2 \%$ & $53.4 \%$ & $46.3 \%$ \\
\hline Dagana & $67.2 \%$ & $65.3 \%$ & $30.5 \%$ & $79.9 \%$ & $61.1 \%$ & $67.7 \%$ & $93.9 \%$ & $97.6 \%$ & $79.8 \%$ & $60.8 \%$ & $49.0 \%$ & $99.7 \%$ & $80.0 \%$ & $54.8 \%$ & $42.6 \%$ \\
\hline Gasa & $80.1 \%$ & $60.6 \%$ & $30.8 \%$ & $71.5 \%$ & $45.1 \%$ & $77.4 \%$ & $94.3 \%$ & $98.2 \%$ & $59.6 \%$ & $89.0 \%$ & $61.6 \%$ & $96.9 \%$ & $78.0 \%$ & $73.2 \%$ & $17.8 \%$ \\
\hline Наa & $77.4 \%$ & $66.5 \%$ & $52.2 \%$ & $55.7 \%$ & $46.1 \%$ & $63.4 \%$ & $94.4 \%$ & $95.2 \%$ & $67.2 \%$ & $87.5 \%$ & $50.3 \%$ & $97.0 \%$ & $71.1 \%$ & $73.3 \%$ & $50.7 \%$ \\
\hline Lhuntse & $79.1 \%$ & $46.9 \%$ & $29.3 \%$ & $65.1 \%$ & $54.1 \%$ & $77.2 \%$ & $89.4 \%$ & $98.7 \%$ & $73.9 \%$ & $86.1 \%$ & $42.8 \%$ & $99.7 \%$ & $68.2 \%$ & $22.3 \%$ & $15.6 \%$ \\
\hline Mongar & $84.9 \%$ & $62.0 \%$ & $39.5 \%$ & $67.3 \%$ & $47.5 \%$ & $75.9 \%$ & $91.8 \%$ & $95.6 \%$ & $78.9 \%$ & $86.5 \%$ & $51.6 \%$ & $93.6 \%$ & $69.4 \%$ & $39.3 \%$ & $23.2 \%$ \\
\hline Paro & $78.4 \%$ & $65.2 \%$ & $49.9 \%$ & $51.1 \%$ & $44.4 \%$ & $60.0 \%$ & $94.4 \%$ & $94.5 \%$ & $85.8 \%$ & $83.5 \%$ & $79.2 \%$ & $88.2 \%$ & $72.9 \%$ & $72.0 \%$ & $63.8 \%$ \\
\hline Tashiyangtse & $83.3 \%$ & $58.3 \%$ & $41.6 \%$ & $73.7 \%$ & $53.8 \%$ & $70.7 \%$ & $96.1 \%$ & $97.2 \%$ & $87.4 \%$ & $86.9 \%$ & $32.8 \%$ & $98.9 \%$ & $56.6 \%$ & $32.1 \%$ & $20.2 \%$ \\
\hline Tashigang & $85.0 \%$ & $51.2 \%$ & $38.9 \%$ & $73.2 \%$ & $64.2 \%$ & $73.0 \%$ & $92.7 \%$ & $98.2 \%$ & $66.8 \%$ & $80.1 \%$ & $33.6 \%$ & $94.3 \%$ & $55.8 \%$ & $28.5 \%$ & $20.3 \%$ \\
\hline Thimphu & $73.9 \%$ & $61.6 \%$ & $70.4 \%$ & $12.4 \%$ & $23.5 \%$ & $34.9 \%$ & $92.5 \%$ & $92.7 \%$ & $61.8 \%$ & $84.6 \%$ & $96.2 \%$ & $35.7 \%$ & $73.9 \%$ & $85.8 \%$ & $77.3 \%$ \\
\hline Trongs & $80.2 \%$ & $57.8 \%$ & $30.8 \%$ & $58.9 \%$ & $41.9 \%$ & $58.8 \%$ & $88.2 \%$ & $95.9 \%$ & $67.6 \%$ & $81.4 \%$ & $43.8 \%$ & $87.6 \%$ & $69.4 \%$ & $51.6 \%$ & $44.6 \%$ \\
\hline Tsirang & $85.4 \%$ & $80.8 \%$ & $21.5 \%$ & $79.7 \%$ & $55.1 \%$ & $81.8 \%$ & $93.6 \%$ & $96.5 \%$ & $72.9 \%$ & $91.4 \%$ & $37.5 \%$ & $97.3 \%$ & $86.4 \%$ & $48.1 \%$ & $47.1 \%$ \\
\hline
\end{tabular}


Karma Ura, Sabina Alkire, Tshoki Zangmo \& Karma Wangdi

\begin{tabular}{|c|c|c|c|c|c|c|c|c|c|c|c|c|c|c|c|}
\hline Wangdue Phodrang & $79.5 \%$ & $63.0 \%$ & $29.1 \%$ & $66.8 \%$ & $49.1 \%$ & $61.2 \%$ & $91.8 \%$ & $97.0 \%$ & $55.0 \%$ & $85.1 \%$ & $50.9 \%$ & $80.9 \%$ & $83.9 \%$ & $55.2 \%$ & $39.7 \%$ \\
\hline \multicolumn{16}{|l|}{ Region } \\
\hline Rural & $81.3 \%$ & $62.5 \%$ & $30.0 \%$ & $72.0 \%$ & $54.3 \%$ & $72.1 \%$ & $92.9 \%$ & $96.7 \%$ & $73.2 \%$ & $84.2 \%$ & $41.8 \%$ & $97.9 \%$ & $72.8 \%$ & $40.8 \%$ & $33.6 \%$ \\
\hline Urban & $74.6 \%$ & $61.3 \%$ & $70.3 \%$ & $14.7 \%$ & $24.6 \%$ & $36.6 \%$ & $92.9 \%$ & $94.8 \%$ & $58.4 \%$ & $84.4 \%$ & $99.1 \%$ & $49.7 \%$ & $77.7 \%$ & $87.3 \%$ & $80.3 \%$ \\
\hline Male & $79.7 \%$ & $65.6 \%$ & $38.5 \%$ & $63.7 \%$ & $52.3 \%$ & $66.7 \%$ & $94.8 \%$ & $96.8 \%$ & $69.5 \%$ & $86.5 \%$ & $54.1 \%$ & $86.4 \%$ & $76.8 \%$ & $50.8 \%$ & $44.4 \%$ \\
\hline Female & $79.2 \%$ & $58.8 \%$ & $43.4 \%$ & $49.3 \%$ & $40.3 \%$ & $58.3 \%$ & $91.1 \%$ & $95.6 \%$ & $68.9 \%$ & $82.0 \%$ & $60.6 \%$ & $83.3 \%$ & $71.5 \%$ & $56.1 \%$ & $48.2 \%$ \\
\hline \multicolumn{16}{|l|}{ Age group } \\
\hline $31-35$ & $79.7 \%$ & $58.4 \%$ & $41.5 \%$ & $56.9 \%$ & $43.1 \%$ & $57.9 \%$ & $93.4 \%$ & $96.2 \%$ & $69.2 \%$ & $85.4 \%$ & $62.8 \%$ & $83.6 \%$ & $77.0 \%$ & $51.6 \%$ & $49.3 \%$ \\
\hline $36-40$ & $78.1 \%$ & $58.8 \%$ & $39.5 \%$ & $57.9 \%$ & $50.1 \%$ & $60.3 \%$ & $91.8 \%$ & $96.9 \%$ & $66.0 \%$ & $86.4 \%$ & $60.0 \%$ & $79.4 \%$ & $73.8 \%$ & $54.8 \%$ & $48.1 \%$ \\
\hline $41-45$ & $78.0 \%$ & $68.9 \%$ & $40.5 \%$ & $62.7 \%$ & $56.6 \%$ & $70.8 \%$ & $92.5 \%$ & $96.8 \%$ & $68.3 \%$ & $83.5 \%$ & $52.7 \%$ & $87.1 \%$ & $76.4 \%$ & $49.4 \%$ & $42.6 \%$ \\
\hline $46-50$ & $82.7 \%$ & $62.2 \%$ & $35.3 \%$ & $72.0 \%$ & $56.7 \%$ & $74.5 \%$ & $95.6 \%$ & $97.5 \%$ & $70.0 \%$ & $86.1 \%$ & $41.6 \%$ & $90.5 \%$ & $78.5 \%$ & $45.2 \%$ & $37.9 \%$ \\
\hline $51-55$ & $82.9 \%$ & $64.4 \%$ & $35.5 \%$ & $73.3 \%$ & $54.7 \%$ & $75.8 \%$ & $95.6 \%$ & $97.9 \%$ & $70.2 \%$ & $85.0 \%$ & $39.9 \%$ & $94.3 \%$ & $74.3 \%$ & $47.3 \%$ & $34.2 \%$ \\
\hline $56-60$ & $83.1 \%$ & $64.1 \%$ & $36.1 \%$ & $77.6 \%$ & $52.2 \%$ & $78.0 \%$ & $95.0 \%$ & $97.8 \%$ & $69.5 \%$ & $85.7 \%$ & $39.1 \%$ & $93.8 \%$ & $74.9 \%$ & $46.1 \%$ & $35.8 \%$ \\
\hline
\end{tabular}


An Extensive Analysis of GNH Index

Table V-A Contribution to overall unhappiness The table shows which dimensions contribute most to a country's GNH Index score

Suggested citation: Ura, Karma; Alkire, Sabina; and Zangmo, Tshoki (Nov 2011) www.grossnationalhappiness.com ophi.qeh.ox.ac.uk

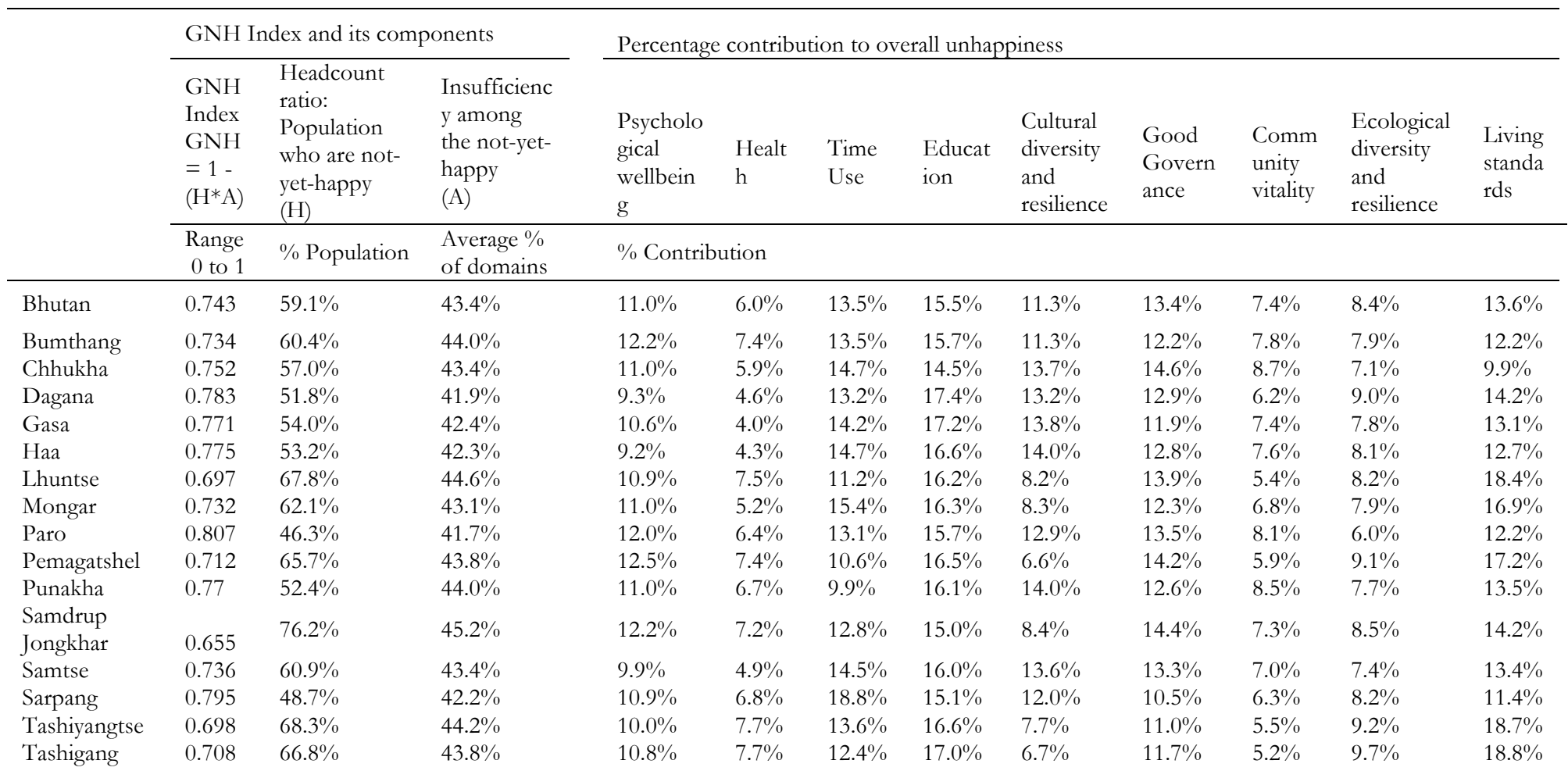


Karma Ura, Sabina Alkire, Tshoki Zangmo \& Karma Wangdi

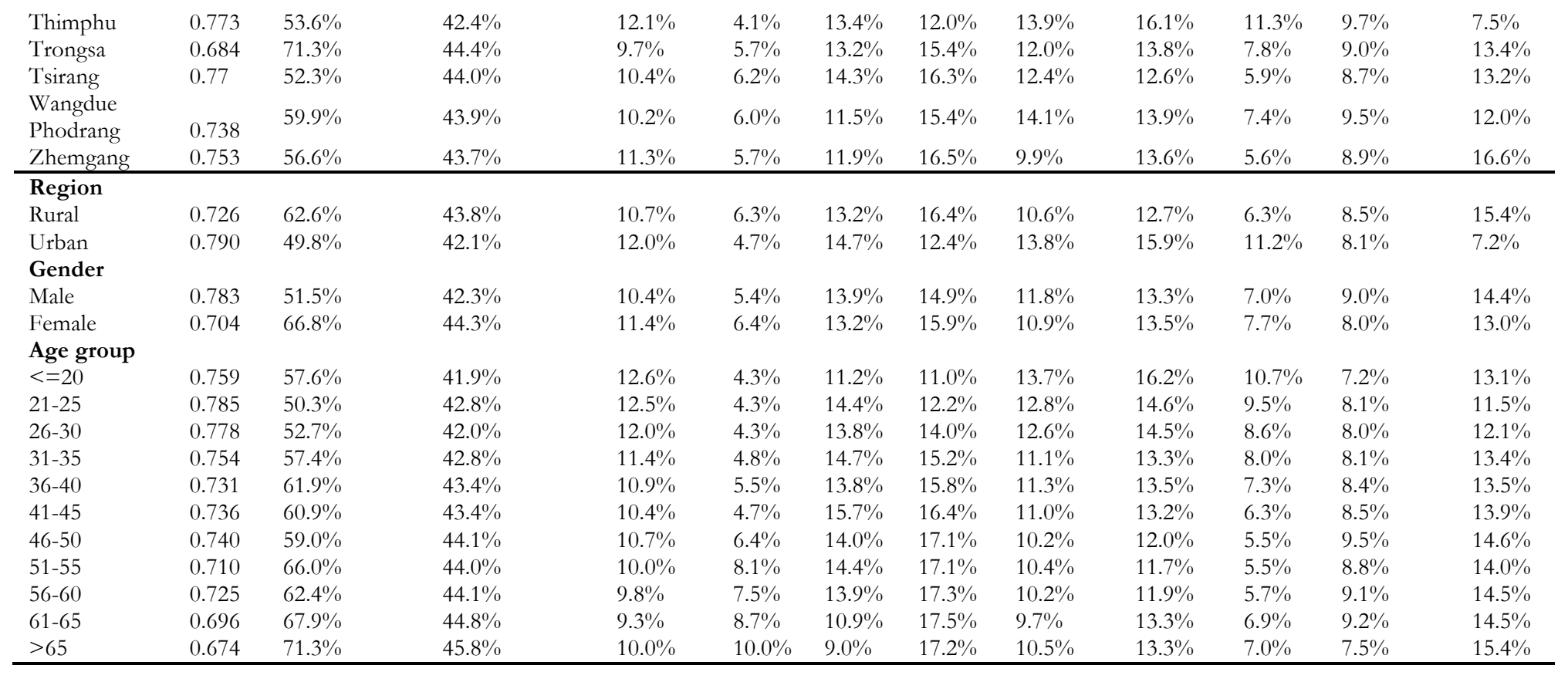


An Extensive Analysis of GNH Index

Table V-B Contribution to overall unhappiness The table shows which dimensions contribute most to a country's GNH Index score

Suggested citation: Ura, Karma; Alkire, Sabina; and Zangmo, Tshoki (Nov 2011) www.grossnationalhappiness.com ophi.qeh.ox.ac.uk

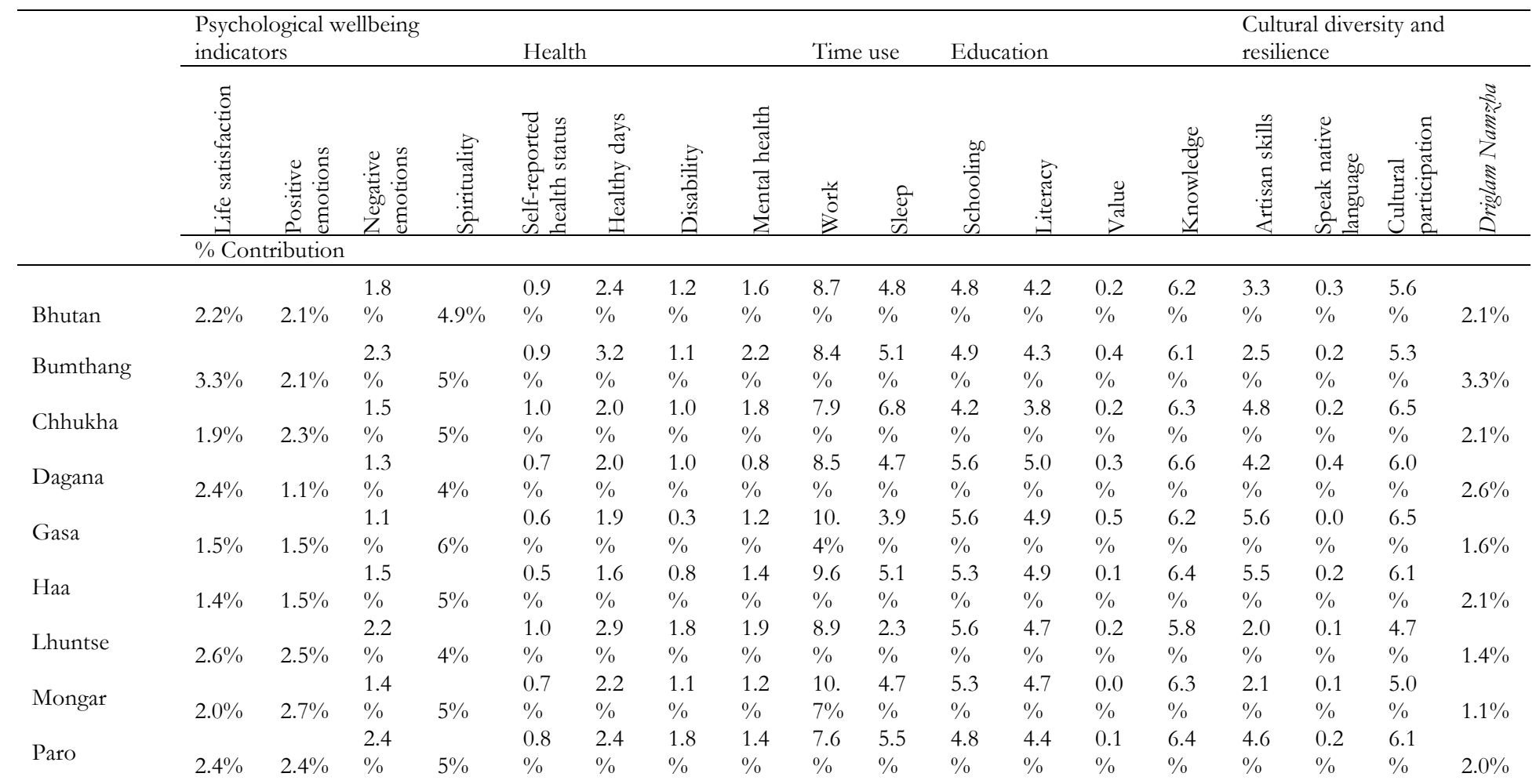


Karma Ura, Sabina Alkire, Tshoki Zangmo \& Karma Wangdi

\begin{tabular}{|c|c|c|c|c|c|c|c|c|c|c|c|c|c|c|c|c|c|c|}
\hline Pemagatshel & $2.5 \%$ & $2.7 \%$ & $\begin{array}{l}1.7 \\
\%\end{array}$ & $6 \%$ & $\begin{array}{l}1.0 \\
\%\end{array}$ & $\begin{array}{l}2.9 \\
\%\end{array}$ & $\begin{array}{l}1.6 \\
\%\end{array}$ & $\begin{array}{l}2.1 \\
\%\end{array}$ & $\begin{array}{l}8.8 \\
\%\end{array}$ & $\begin{array}{l}1.7 \\
\%\end{array}$ & $\begin{array}{l}5.5 \\
\%\end{array}$ & $\begin{array}{l}4.5 \\
\%\end{array}$ & $\begin{array}{l}0.2 \\
\%\end{array}$ & $\begin{array}{l}6.3 \\
\%\end{array}$ & $\begin{array}{l}1.2 \\
\%\end{array}$ & $\begin{array}{l}0.1 \\
\%\end{array}$ & $\begin{array}{l}4.0 \\
\%\end{array}$ & $1.3 \%$ \\
\hline \multirow{2}{*}{ Punakha } & & & 2.3 & & 1.0 & 2.3 & 1.4 & 1.9 & 5.5 & 4.4 & 5.3 & 4.8 & 0.0 & 6.0 & 5.3 & 0.1 & 6.3 & \\
\hline & $2.4 \%$ & $1.6 \%$ & $\%$ & $5 \%$ & $\%$ & $\%$ & $\%$ & $\%$ & $\%$ & $\%$ & $\%$ & $\%$ & $\%$ & $\%$ & $\%$ & $\%$ & $\%$ & $2.3 \%$ \\
\hline Samdrup & & & 2.3 & & 0.8 & 3.3 & 1.3 & 1.8 & 9.4 & 3.5 & 4.8 & 4.0 & 0.2 & 6.0 & 1.5 & 0.2 & 4.2 & \\
\hline Jongkhar & $3.0 \%$ & $2.4 \%$ & $\%$ & $4 \%$ & $\%$ & $\%$ & $\%$ & $\%$ & $\%$ & $\%$ & $\%$ & $\%$ & $\%$ & $\%$ & $\%$ & $\%$ & $\%$ & $2.4 \%$ \\
\hline \multirow{2}{*}{ Samtse } & & & 1.1 & & 0.9 & 2.0 & 1.0 & 1.0 & 8.8 & 5.7 & 5.3 & 4.2 & 0.1 & 6.4 & 4.3 & 0.5 & 7.0 & \\
\hline & $1.5 \%$ & $2.1 \%$ & $\%$ & $5 \%$ & $\%$ & $\%$ & $\%$ & $\%$ & $\%$ & $\%$ & $\%$ & $\%$ & $\%$ & $\%$ & $\%$ & $\%$ & $\%$ & $1.9 \%$ \\
\hline \multirow{2}{*}{ Sarpang } & & & 1.5 & & 1.4 & 2.7 & 1.0 & 1.7 & 10. & 8.7 & 4.5 & 4.1 & 0.1 & 6.5 & 3.3 & 0.8 & 5.9 & \\
\hline & $2.9 \%$ & $2.0 \%$ & $\%$ & $4 \%$ & $\%$ & $\%$ & $\%$ & $\%$ & $1 \%$ & $\%$ & $\%$ & $\%$ & $\%$ & $\%$ & $\%$ & $\%$ & $\%$ & $2.0 \%$ \\
\hline \multirow{2}{*}{ Tashiyangtse } & & & 2.3 & & 0.9 & 3.1 & 1.8 & 1.9 & 10. & 2.7 & 5.5 & 4.8 & 0.2 & 6.1 & 1.8 & 0.1 & 4.6 & \\
\hline & $2.4 \%$ & $1.8 \%$ & $\%$ & $4 \%$ & $\%$ & $\%$ & $\%$ & $\%$ & $8 \%$ & $\%$ & $\%$ & $\%$ & $\%$ & $\%$ & $\%$ & $\%$ & $\%$ & $1.1 \%$ \\
\hline \multirow{2}{*}{ Tashigang } & & & 2.3 & & 0.9 & 3.3 & 1.2 & 2.2 & 9.5 & 2.9 & 5.7 & 5.0 & 0.1 & 6.2 & 1.1 & 0.2 & 4.0 & \\
\hline & $2.4 \%$ & $1.8 \%$ & $\%$ & $4 \%$ & $\%$ & $\%$ & $\%$ & $\%$ & $\%$ & $\%$ & $\%$ & $\%$ & $\%$ & $\%$ & $\%$ & $\%$ & $\%$ & $1.5 \%$ \\
\hline \multirow{2}{*}{ Thimphu } & & & 2.0 & & 0.6 & 1.6 & 0.9 & 1.0 & 7.6 & 5.8 & 2.9 & 2.5 & 0.5 & 6.2 & 3.9 & 0.5 & 6.5 & \\
\hline & $2.0 \%$ & $2.4 \%$ & $\%$ & $6 \%$ & $\%$ & $\%$ & $\%$ & $\%$ & $\%$ & $\%$ & $\%$ & $\%$ & $\%$ & $\%$ & $\%$ & $\%$ & $\%$ & $3.0 \%$ \\
\hline \multirow{2}{*}{ Trongsa } & & & 2.0 & & $\begin{array}{l}0.7 \\
0 \%\end{array}$ & $\begin{array}{l}2.5 \\
0 \%\end{array}$ & 0.8 & $\begin{array}{l}1.7 \\
0 \%\end{array}$ & 8.7 & 4.5 & 4.8 & 4.4 & 0.1 & 6.0 & 3.9 & 0.2 & 5.2 & \\
\hline & $2.1 \%$ & $1.9 \%$ & $\%$ & $4 \%$ & $\%$ & $\%$ & $\%$ & $\%$ & $\%$ & $\%$ & $\%$ & $\%$ & $\%$ & $\%$ & $\%$ & $\%$ & $\%$ & $2.8 \%$ \\
\hline \multirow{2}{*}{ Tsirang } & & & 1.6 & & 1.1 & 2.2 & 1.4 & 1.5 & 9.3 & 5.0 & 4.9 & 5.1 & 0.0 & 6.3 & 3.8 & 0.4 & 5.9 & \\
\hline & $2.7 \%$ & $1.5 \%$ & $\%$ & $5 \%$ & $\%$ & $\%$ & $\%$ & $\%$ & $\%$ & $\%$ & $\%$ & $\%$ & $\%$ & $\%$ & $\%$ & $\%$ & $\%$ & $2.3 \%$ \\
\hline Wangdue & & & 1.7 & & 0.7 & 2.3 & 1.1 & 1.9 & 7.9 & 3.6 & 4.8 & 4.3 & 0.1 & 6.2 & 5.0 & 0.2 & 6.5 & \\
\hline Phodrang & $1.5 \%$ & $1.9 \%$ & $\%$ & $5 \%$ & $\%$ & $\%$ & $\%$ & $\%$ & $\%$ & $\%$ & $\%$ & $\%$ & $\%$ & $\%$ & $\%$ & $\%$ & $\%$ & $2.4 \%$ \\
\hline \multirow{2}{*}{ Zhemgang } & & & 1.8 & & 1.1 & 2.3 & 1.0 & 1.3 & 7.5 & 4.4 & 5.3 & 4.8 & 0.0 & 6.3 & 2.3 & 0.2 & 5.6 & \\
\hline & $2.2 \%$ & $1.8 \%$ & $\%$ & $5 \%$ & $\%$ & $\%$ & $\%$ & $\%$ & $\%$ & $\%$ & $\%$ & $\%$ & $\%$ & $\%$ & $\%$ & $\%$ & $\%$ & $1.8 \%$ \\
\hline \multicolumn{19}{|l|}{ Region } \\
\hline & & & 1.7 & & 0.9 & 2.5 & 1.3 & 1.7 & 9.0 & 4.2 & 5.3 & 4.7 & 0.1 & 6.2 & 3.2 & 0.2 & 5.4 & \\
\hline \multirow[t]{2}{*}{ Rural } & $2.2 \%$ & $2.1 \%$ & $\%$ & $4.6 \%$ & $\%$ & $\%$ & $\%$ & $\%$ & $\%$ & $\%$ & $\%$ & $\%$ & $\%$ & $\%$ & $\%$ & $\%$ & $\%$ & $1.8 \%$ \\
\hline & & & 2.0 & & 0.7 & 1.9 & 0.9 & 1.2 & 7.7 & 6.9 & 3.0 & 2.6 & 0.5 & 6.3 & 3.6 & 0.4 & 6.6 & \\
\hline Urban & $2.0 \%$ & $2.3 \%$ & $\%$ & $5.7 \%$ & $\%$ & $\%$ & $\%$ & $\%$ & $\%$ & $\%$ & $\%$ & $\%$ & $\%$ & $\%$ & $\%$ & $\%$ & $\%$ & $3.1 \%$ \\
\hline
\end{tabular}

Gender 
An Extensive Analysis of GNH Index

\begin{tabular}{|c|c|c|c|c|c|c|c|c|c|c|c|c|c|c|c|c|c|c|}
\hline \multirow{3}{*}{ Male } & \multirow{3}{*}{$2.0 \%$} & \multirow{3}{*}{$2.2 \%$} & \multicolumn{2}{|l|}{1.5} & \multirow{3}{*}{$\begin{array}{l}0.8 \\
\%\end{array}$} & \multirow{2}{*}{$\begin{array}{l}2.1 \\
\%\end{array}$} & \multirow{2}{*}{$\begin{array}{l}1.2 \\
\%\end{array}$} & \multirow{2}{*}{$\begin{array}{l}1.3 \\
\%\end{array}$} & \multirow{3}{*}{$\begin{array}{l}8.6 \\
\% \\
8.9\end{array}$} & \multirow{3}{*}{$\begin{array}{l}5.3 \\
\% \\
4.4\end{array}$} & \multirow{3}{*}{$\begin{array}{l}4.6 \\
\% \\
50\end{array}$} & \multirow{3}{*}{$\begin{array}{l}3.7 \\
\% \\
4.6\end{array}$} & \multirow{3}{*}{$\begin{array}{l}0.2 \\
\% \\
0.1\end{array}$} & \multirow{3}{*}{$\begin{array}{l}6.3 \\
\% \\
6.2\end{array}$} & \multirow{3}{*}{$\begin{array}{l}3.5 \\
\% \\
3.2\end{array}$} & \multirow{3}{*}{$\begin{array}{l}0.4 \\
\% \\
0.2\end{array}$} & \multirow{3}{*}{$\begin{array}{l}5.8 \\
\% \\
5.6\end{array}$} & \multirow{2}{*}{$2.2 \%$} \\
\hline & & & $\%$ & $4.7 \%$ & & & & & & & & & & & & & & \\
\hline & & & 2.0 & & & 2.6 & 1.1 & 1.8 & & & & & & & & & & \\
\hline Female & $2.4 \%$ & $2.1 \%$ & $\%$ & $5.0 \%$ & $\%$ & $\%$ & $\%$ & $\%$ & $\%$ & $\%$ & $\%$ & $\%$ & $\%$ & $\%$ & $\%$ & $\%$ & $\%$ & $2.0 \%$ \\
\hline \multicolumn{19}{|l|}{ Age group } \\
\hline \multirow{3}{*}{$<=20$} & & & 2.4 & & 0.6 & 1.9 & 0.4 & 1.4 & 5.5 & 5.6 & 2.3 & 1.8 & 0.3 & 6.5 & 3.7 & 0.8 & 7.1 & \\
\hline & $1.4 \%$ & $2.5 \%$ & $\%$ & $6.2 \%$ & $\%$ & $\%$ & $\%$ & $\%$ & $\%$ & $\%$ & $\%$ & $\%$ & $\%$ & $\%$ & $\%$ & $\%$ & $\%$ & $2.2 \%$ \\
\hline & & & 2.3 & & 0.6 & 2.1 & 0.4 & 1.3 & 8.9 & 5.5 & 3.2 & 2.3 & 0.5 & 6.2 & 3.7 & 0.4 & 6.1 & \\
\hline \multirow[t]{2}{*}{$21-25$} & $1.7 \%$ & $2.4 \%$ & $\%$ & $6.2 \%$ & $\%$ & $\%$ & $\%$ & $\%$ & $\%$ & $\%$ & $\%$ & $\%$ & $\%$ & $\%$ & $\%$ & $\%$ & $\%$ & $2.6 \%$ \\
\hline & & & 2.0 & & 0.6 & 1.9 & 0.7 & 1.0 & 9.1 & 4.7 & 3.9 & 3.5 & 0.2 & 6.4 & 3.6 & 0.4 & 6.1 & \\
\hline \multirow[t]{2}{*}{$26-30$} & $1.6 \%$ & $2.3 \%$ & $\%$ & $6.1 \%$ & $\%$ & $\%$ & $\%$ & $\%$ & $\%$ & $\%$ & $\%$ & $\%$ & $\%$ & $\%$ & $\%$ & $\%$ & $\%$ & $2.6 \%$ \\
\hline & & & 1.6 & & 0.7 & 2.4 & 0.7 & 1.1 & 9.8 & 4.9 & 4.7 & 4.0 & 0.1 & 6.4 & 2.9 & 0.2 & 5.8 & \\
\hline \multirow{2}{*}{$31-35$} & $1.8 \%$ & $2.3 \%$ & $\%$ & $5.6 \%$ & $\%$ & $\%$ & $\%$ & $\%$ & $\%$ & $\%$ & $\%$ & $\%$ & $\%$ & $\%$ & $\%$ & $\%$ & $\%$ & $2.2 \%$ \\
\hline & & & 1.9 & & 0.8 & 2.2 & 1.0 & 1.5 & 9.1 & 4.8 & 4.9 & 4.4 & 0.3 & 6.2 & 3.2 & 0.3 & 5.7 & \\
\hline \multirow[t]{2}{*}{$36-40$} & $2.1 \%$ & $2.1 \%$ & $\%$ & $4.9 \%$ & $\%$ & $\%$ & $\%$ & $\%$ & $\%$ & $\%$ & $\%$ & $\%$ & $\%$ & $\%$ & $\%$ & $\%$ & $\%$ & $2.0 \%$ \\
\hline & & & 1.5 & & 0.7 & 2.1 & 1.0 & 0.9 & 10. & 5.4 & 5.2 & 4.8 & 0.1 & 6.2 & 3.5 & 0.3 & 5.2 & \\
\hline \multirow[t]{2}{*}{$41-45$} & $2.1 \%$ & $2.1 \%$ & $\%$ & $4.8 \%$ & $\%$ & $\%$ & $\%$ & $\%$ & $3 \%$ & $\%$ & $\%$ & $\%$ & $\%$ & $\%$ & $\%$ & $\%$ & $\%$ & $2.0 \%$ \\
\hline & & & 1.8 & & 0.9 & 2.2 & 1.3 & 1.9 & 9.3 & 4.6 & 5.7 & 5.0 & 0.2 & 6.2 & 3.0 & 0.1 & 5.4 & \\
\hline \multirow[t]{2}{*}{$46-50$} & $2.5 \%$ & $2.1 \%$ & $\%$ & $4.3 \%$ & $\%$ & $\%$ & $\%$ & $\%$ & $\%$ & $\%$ & $\%$ & $\%$ & $\%$ & $\%$ & $\%$ & $\%$ & $\%$ & $1.7 \%$ \\
\hline & & & 1.6 & & 1.1 & 3.1 & 2.0 & 1.9 & 9.3 & 5.0 & 5.6 & 5.2 & 0.1 & 6.2 & 2.8 & 0.4 & 5.4 & \\
\hline $51-55$ & $2.5 \%$ & $2.2 \%$ & $\%$ & $3.7 \%$ & $\%$ & $\%$ & $\%$ & $\%$ & $\%$ & $\%$ & $\%$ & $\%$ & $\%$ & $\%$ & $\%$ & $\%$ & $\%$ & $1.8 \%$ \\
\hline & & & 1.5 & & 1.1 & 2.5 & 1.8 & 2.0 & 9.1 & 4.8 & 5.8 & 5.2 & 0.1 & 6.2 & 3.1 & 0.2 & 5.6 & \\
\hline $56-60$ & $2.4 \%$ & $2.0 \%$ & $\%$ & $3.8 \%$ & $\%$ & $\%$ & $\%$ & $\%$ & $\%$ & $\%$ & $\%$ & $\%$ & $\%$ & $\%$ & $\%$ & $\%$ & $\%$ & $1.3 \%$ \\
\hline & & & 1.7 & & 1.1 & 3.6 & 1.8 & 2.2 & 7.3 & 3.6 & 6.0 & 5.3 & 0.0 & 6.2 & 3.5 & 0.1 & 4.5 & \\
\hline $61-65$ & $2.7 \%$ & $1.6 \%$ & $\%$ & $3.3 \%$ & $\%$ & $\%$ & $\%$ & $\%$ & $\%$ & $\%$ & $\%$ & $\%$ & $\%$ & $\%$ & $\%$ & $\%$ & $\%$ & $1.6 \%$ \\
\hline & & & 1.4 & & 1.5 & 3.3 & 2.3 & 2.9 & 5.9 & 3.2 & 6.0 & 5.2 & 0.0 & 6.0 & 3.5 & 0.1 & 5.0 & \\
\hline$>65$ & $3.8 \%$ & $1.8 \%$ & $\%$ & $3.0 \%$ & $\%$ & $\%$ & $\%$ & $\%$ & $\%$ & $\%$ & $\%$ & $\%$ & $\%$ & $\%$ & $\%$ & $\%$ & $\%$ & $1.9 \%$ \\
\hline
\end{tabular}


Karma Ura, Sabina Alkire, Tshoki Zangmo \& Karma Wangdi

Table V-B (cont.) Contribution to overall unhappiness This table shows which dimensions contribute most to a country's GNH Index score

Suggested citation: Ura, Karma; Alkire, Sabina; and Zangmo, Tshoki (Nov 2011) www.grossnationalhappiness.com ophi.qeh.ox.ac.uk

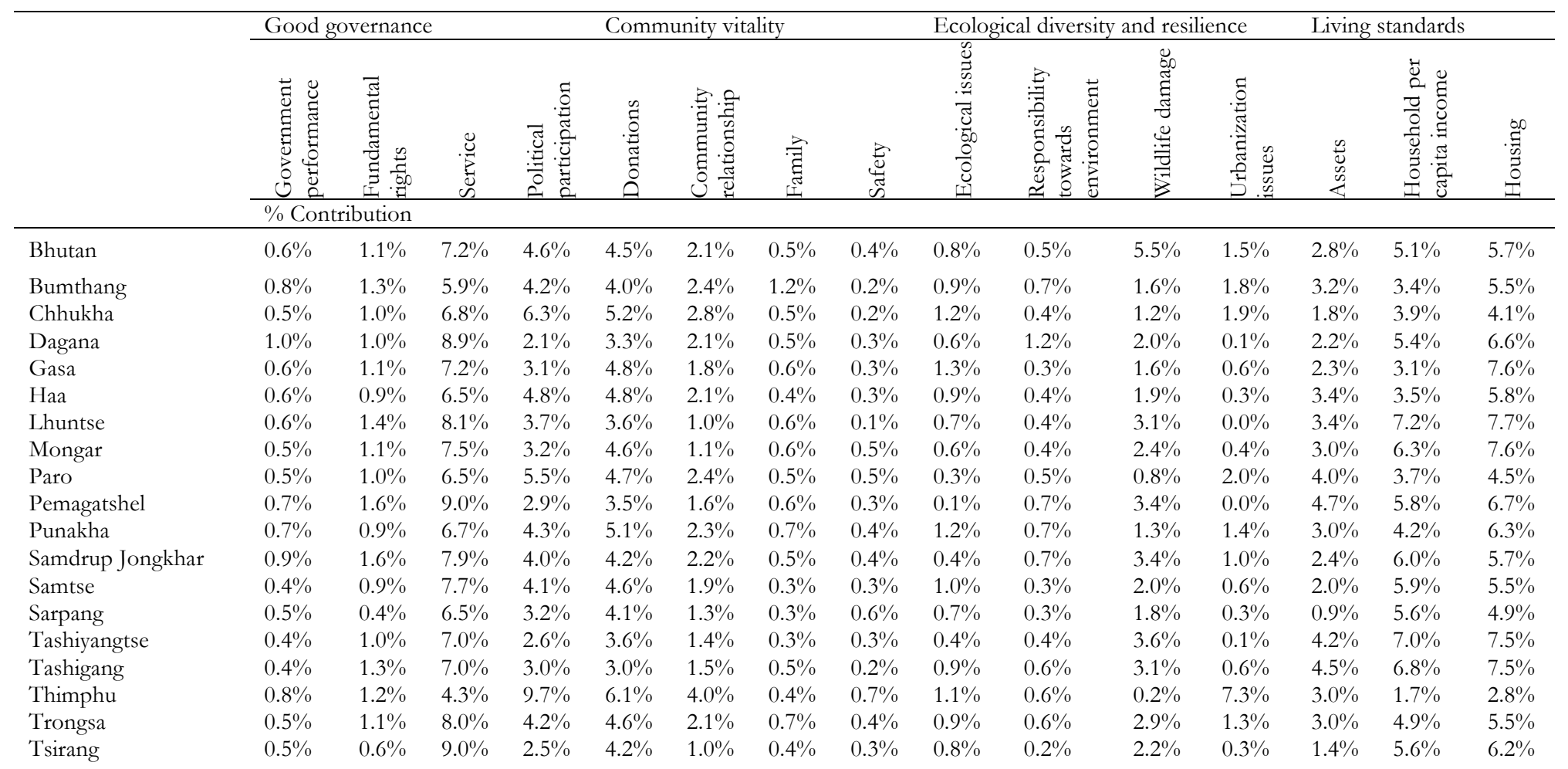

198 
An Extensive Analysis of GNH Index

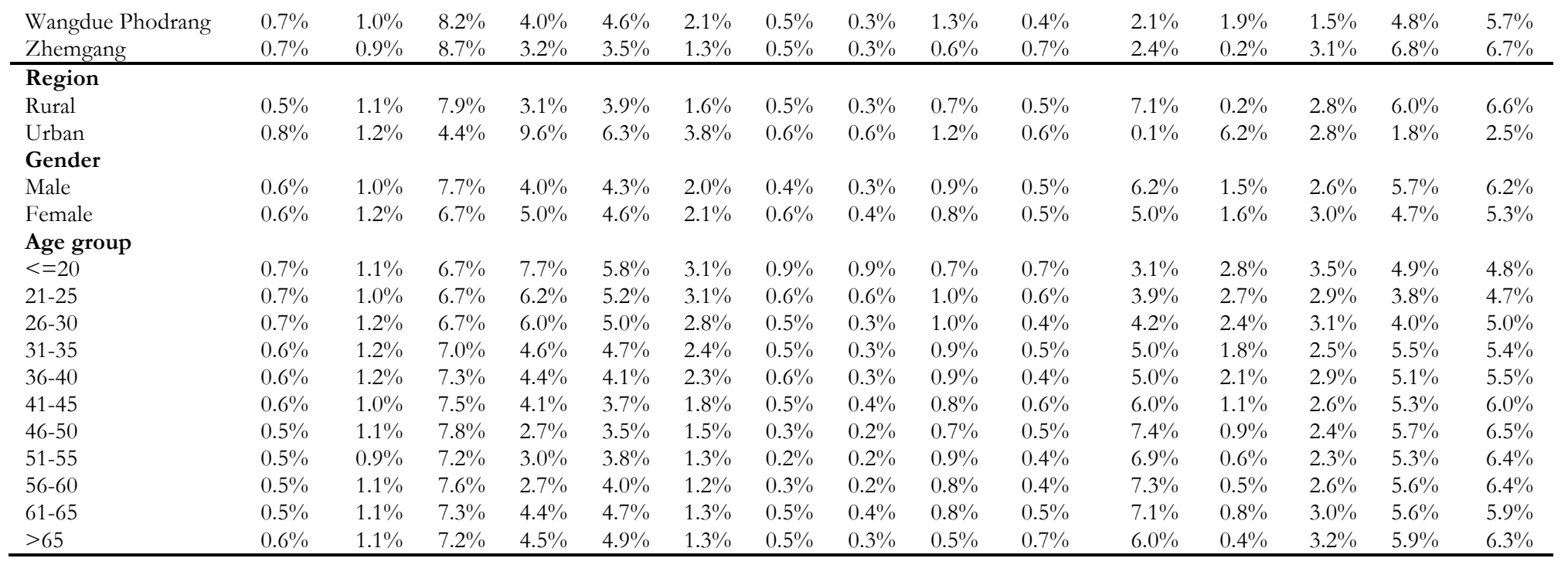


Karma Ura, Sabina Alkire, Tshoki Zangmo \& Karma Wangdi

Table VI Increasing Happiness - Censored headcounts This table shows the proportion of people who are not yet happy and experience insufficiencies in each of the indicators.

Suggested citation: Ura, Karma; Alkire, Sabina; and Zangmo, Tshoki (Nov 2011) www.grossnationalhappiness.com ophi.qeh.ox.ac.uk

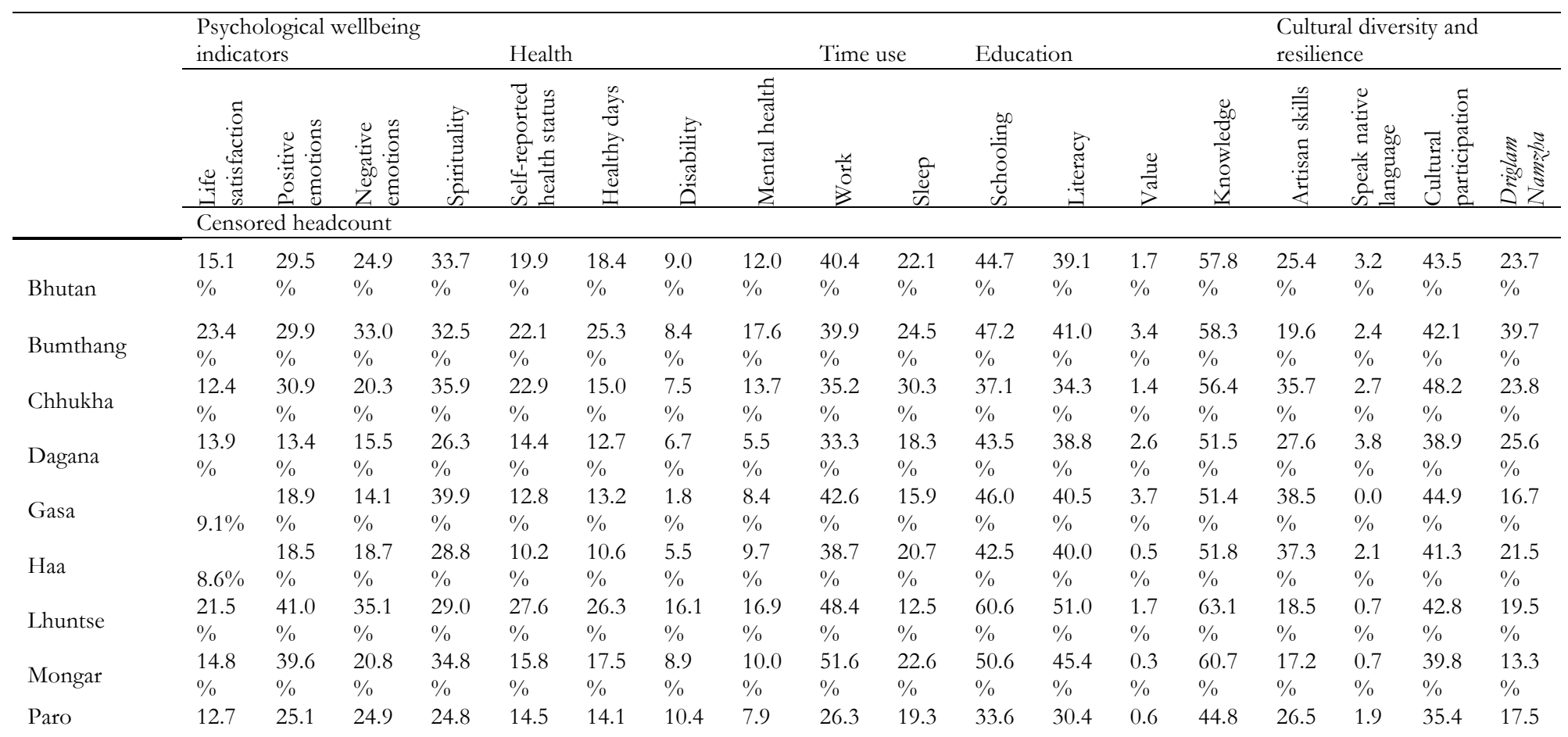

200 
An Extensive Analysis of GNH Index

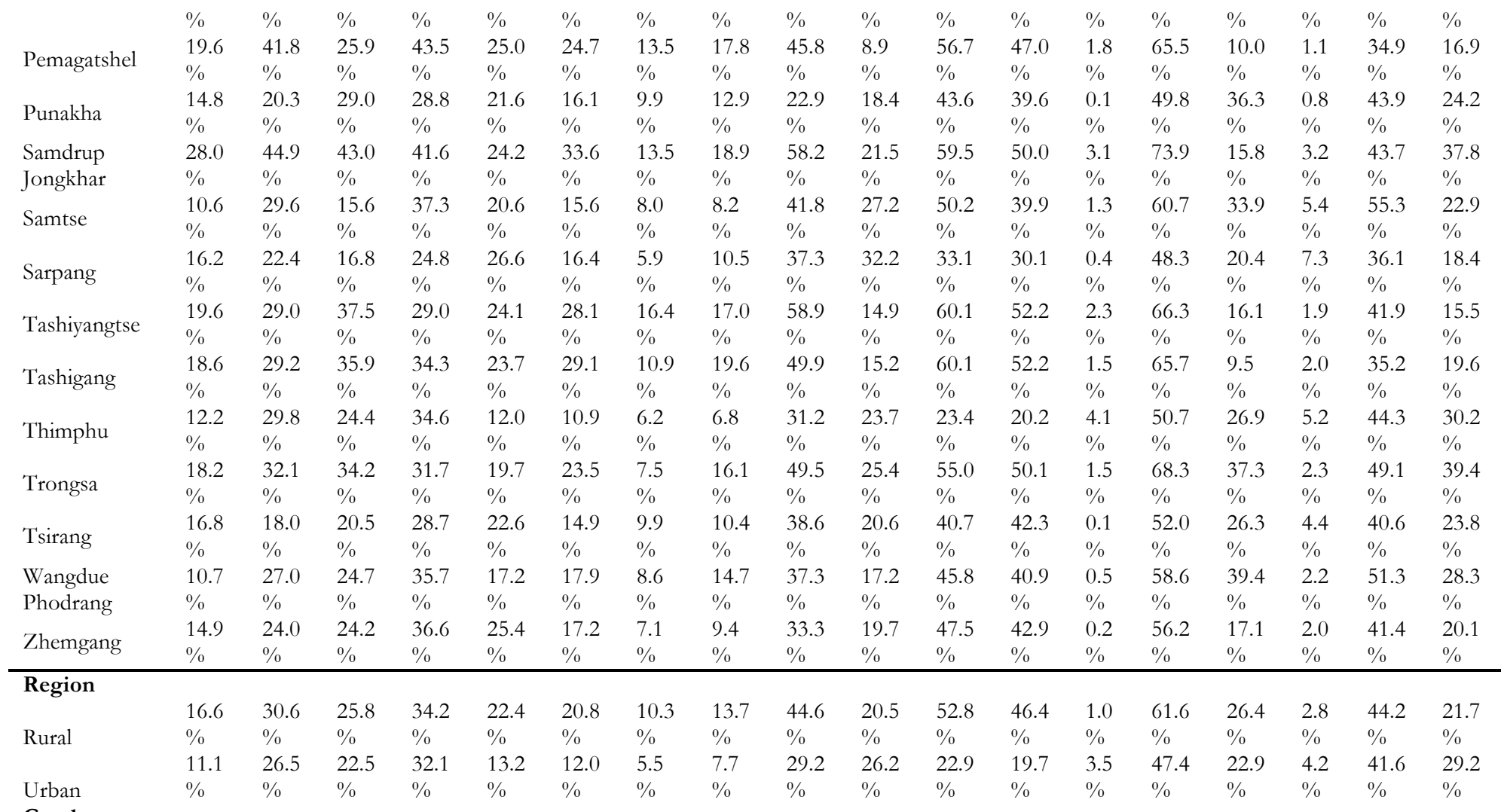


Karma Ura, Sabina Alkire, Tshoki Zangmo \& Karma Wangdi

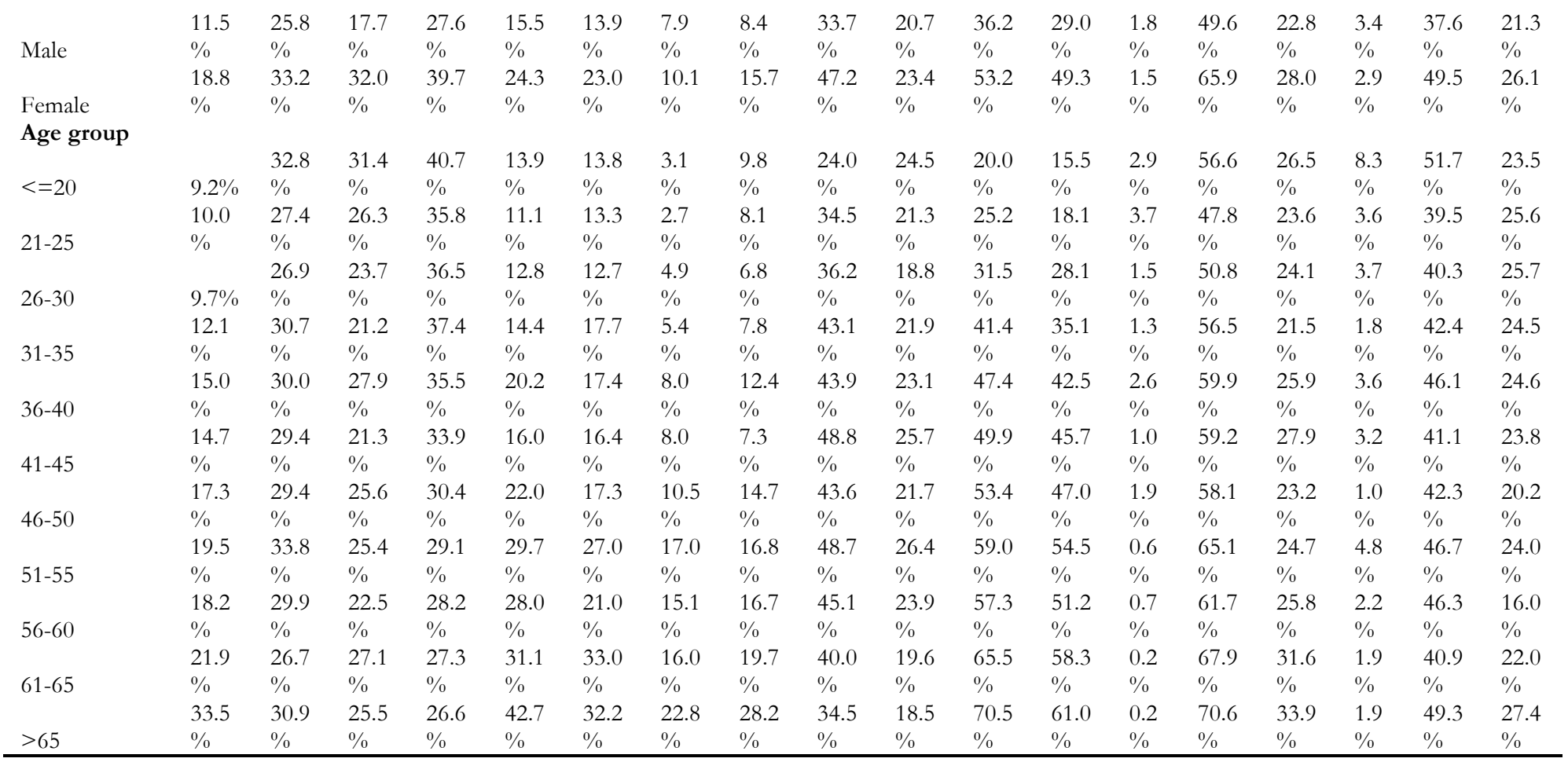


An Extensive Analysis of GNH Index

Table VI (cont.) Increasing Happiness - Censored headcounts This table shows the proportion of people who are not yet happy and experience insufficiencies in each of the indicators.

Suggested citation: Ura, Karma; Alkire, Sabina; and Zangmo, Tshoki (Nov 2011) www.grossnationalhappiness.com ophi.qeh.ox.ac.uk

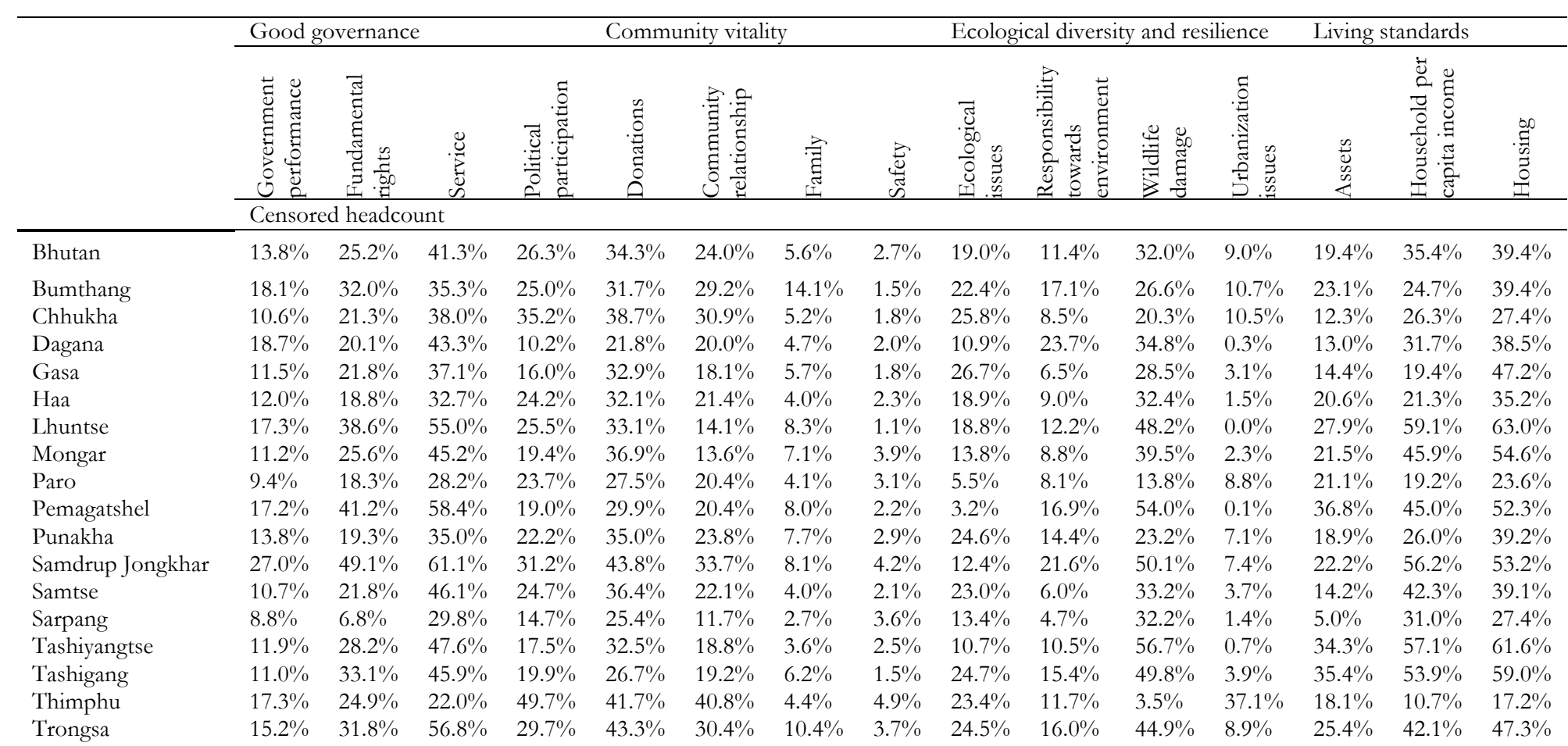


Karma Ura, Sabina Alkire, Tshoki Zangmo \& Karma Wangdi

\begin{tabular}{|c|c|c|c|c|c|c|c|c|c|c|c|c|c|c|c|}
\hline Tsirang & $10.4 \%$ & $11.8 \%$ & $46.8 \%$ & $13.0 \%$ & $29.2 \%$ & $9.9 \%$ & $4.4 \%$ & $1.8 \%$ & $16.5 \%$ & $4.9 \%$ & $38.4 \%$ & $1.4 \%$ & $8.9 \%$ & $34.8 \%$ & $38.3 \%$ \\
\hline Zhemgang & $16.2 \%$ & $19.4 \%$ & $48.6 \%$ & $18.0 \%$ & $26.3 \%$ & $14.5 \%$ & $5.2 \%$ & $2.1 \%$ & $12.3 \%$ & $15.3 \%$ & $41.5 \%$ & $1.1 \%$ & $20.6 \%$ & $45.2 \%$ & $44.7 \%$ \\
\hline \multicolumn{16}{|l|}{ Region } \\
\hline Rural & $13.3 \%$ & $26.0 \%$ & $49.0 \%$ & $19.4 \%$ & $32.4 \%$ & $19.5 \%$ & $5.7 \%$ & $2.5 \%$ & $17.5 \%$ & $11.8 \%$ & $43.7 \%$ & $1.4 \%$ & $20.7 \%$ & $44.7 \%$ & $48.7 \%$ \\
\hline \multicolumn{16}{|l|}{ Gender } \\
\hline Male & $11.6 \%$ & $19.7 \%$ & $37.7 \%$ & $19.6 \%$ & $28.0 \%$ & $19.5 \%$ & $3.8 \%$ & $1.9 \%$ & $16.7 \%$ & $8.9 \%$ & $30.3 \%$ & $7.1 \%$ & $15.2 \%$ & $33.2 \%$ & $36.1 \%$ \\
\hline Female & $16.0 \%$ & $30.7 \%$ & $45.0 \%$ & $33.1 \%$ & $40.6 \%$ & $28.6 \%$ & $7.5 \%$ & $3.6 \%$ & $21.3 \%$ & $14.0 \%$ & $33.7 \%$ & $10.8 \%$ & $23.7 \%$ & $37.5 \%$ & $42.6 \%$ \\
\hline $26-30$ & $14.3 \%$ & $23.4 \%$ & $33.2 \%$ & $29.8 \%$ & $33.2 \%$ & $28.2 \%$ & $4.8 \%$ & $2.1 \%$ & $19.8 \%$ & $8.8 \%$ & $21.0 \%$ & $11.8 \%$ & $18.5 \%$ & $24.1 \%$ & $30.0 \%$ \\
\hline $31-35$ & $12.3 \%$ & $25.8 \%$ & $38.6 \%$ & $25.6 \%$ & $34.9 \%$ & $26.6 \%$ & $5.7 \%$ & $2.5 \%$ & $19.0 \%$ & $10.4 \%$ & $27.4 \%$ & $10.1 \%$ & $16.3 \%$ & $36.8 \%$ & $36.0 \%$ \\
\hline $36-40$ & $15.3 \%$ & $28.7 \%$ & $44.0 \%$ & $26.3 \%$ & $33.1 \%$ & $27.3 \%$ & $7.4 \%$ & $2.6 \%$ & $21.6 \%$ & $9.9 \%$ & $30.4 \%$ & $12.5 \%$ & $21.2 \%$ & $37.0 \%$ & $40.0 \%$ \\
\hline $41-45$ & $14.5 \%$ & $22.6 \%$ & $44.4 \%$ & $24.6 \%$ & $29.5 \%$ & $21.3 \%$ & $5.5 \%$ & $2.8 \%$ & $19.0 \%$ & $13.1 \%$ & $35.9 \%$ & $6.8 \%$ & $18.7 \%$ & $37.8 \%$ & $42.5 \%$ \\
\hline $46-50$ & $11.2 \%$ & $25.3 \%$ & $45.4 \%$ & $15.6 \%$ & $27.5 \%$ & $17.0 \%$ & $3.9 \%$ & $1.5 \%$ & $17.1 \%$ & $10.7 \%$ & $43.1 \%$ & $5.4 \%$ & $17.1 \%$ & $39.9 \%$ & $45.7 \%$ \\
\hline $51-55$ & $12.2 \%$ & $24.8 \%$ & $47.0 \%$ & $19.9 \%$ & $32.9 \%$ & $16.7 \%$ & $3.3 \%$ & $2.1 \%$ & $22.4 \%$ & $10.4 \%$ & $45.3 \%$ & $3.9 \%$ & $18.3 \%$ & $41.8 \%$ & $49.8 \%$ \\
\hline
\end{tabular}


An Extensive Analysis of GNH Index

Table VII Increasing Happiness - Raw headcounts The table shows the proportion of people who experience sufficiency in each of the indicators.

Suggested citation: Ura, Karma; Alkire, Sabina; and Zangmo, Tshoki (Nov 2011) www.grossnationalhappiness.com ophi.qeh.ox.ac.uk

\begin{tabular}{|c|c|c|c|c|c|c|c|c|c|c|c|c|c|c|c|c|c|c|}
\hline & \multicolumn{4}{|c|}{ Psychological wellbeing } & \multicolumn{4}{|c|}{ Health } & \multicolumn{2}{|c|}{ Time use } & \multicolumn{4}{|c|}{ Education } & \multicolumn{4}{|c|}{ Cultural diversity and resilience } \\
\hline & 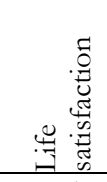 & 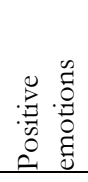 & 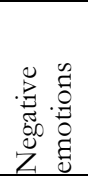 & 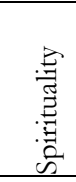 & 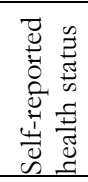 & 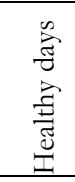 & 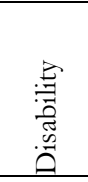 & 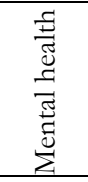 & $\frac{\pi}{0}$ & $\frac{\ddot{\ddot{d}}}{\omega}$ & $\begin{array}{l}60 \\
: \\
: \\
0 \\
0 \\
\bar{y} \\
n \\
\end{array}$ & 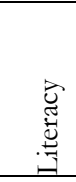 & $\frac{\mathscr{\Xi}}{\frac{\pi}{\pi}}$ & 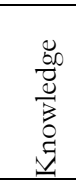 & 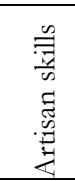 & 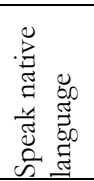 & 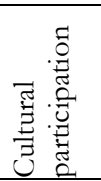 & $\begin{array}{l}8 \\
3 \\
0 \\
0 \\
0\end{array}$ \\
\hline & Raw her & ount & & & & & & & & & & & & & & & & \\
\hline Bhutan & $16.9 \%$ & $\begin{array}{l}41.2 \\
\%\end{array}$ & $\begin{array}{l}35.3 \\
\%\end{array}$ & $\begin{array}{l}47.2 \\
\%\end{array}$ & $\begin{array}{l}26.2 \\
\%\end{array}$ & $\begin{array}{l}23.5 \\
\%\end{array}$ & $\begin{array}{l}10.5 \\
\%\end{array}$ & $\begin{array}{l}13.8 \\
\%\end{array}$ & $\begin{array}{l}55.0 \\
\%\end{array}$ & 332 & $\begin{array}{l}62.6 \\
\%\end{array}$ & $\begin{array}{l}51.7 \\
\%\end{array}$ & $\begin{array}{l}2.7 \\
\%\end{array}$ & $\begin{array}{l}92.6 \\
\%\end{array}$ & $\begin{array}{l}37.9 \\
\%\end{array}$ & $4.9 \%$ & $\begin{array}{l}66.8 \\
\%\end{array}$ & $\begin{array}{l}39.8 \\
\%\end{array}$ \\
\hline Bumthang & $24.8 \%$ & $\begin{array}{l}41.2 \\
\%\end{array}$ & $\begin{array}{l}45.6 \\
\%\end{array}$ & $\begin{array}{l}47.4 \\
\%\end{array}$ & $\begin{array}{l}26.5 \\
\%\end{array}$ & $\begin{array}{l}31.3 \\
\%\end{array}$ & $\begin{array}{l}11.6 \\
\%\end{array}$ & $\begin{array}{l}19.8 \\
\%\end{array}$ & $\begin{array}{l}56.6 \\
\%\end{array}$ & $\begin{array}{l}33.0 \\
\%\end{array}$ & $\begin{array}{l}65.1 \\
\%\end{array}$ & $\begin{array}{l}51.5 \\
\%\end{array}$ & $\begin{array}{l}4.5 \\
\%\end{array}$ & $\begin{array}{l}92.8 \\
\%\end{array}$ & $\begin{array}{l}29.6 \\
\%\end{array}$ & $3.3 \%$ & $\begin{array}{l}62.4 \\
\%\end{array}$ & $\begin{array}{l}66.6 \\
\%\end{array}$ \\
\hline Chhukha & $13.1 \%$ & $\begin{array}{l}41.2 \\
\%\end{array}$ & $\begin{array}{l}27.8 \\
\%\end{array}$ & $\begin{array}{l}48.0 \\
\%\end{array}$ & $\begin{array}{l}27.7 \\
\%\end{array}$ & $\begin{array}{l}20.6 \\
\%\end{array}$ & $8.2 \%$ & $\begin{array}{l}16.8 \\
\%\end{array}$ & $\begin{array}{l}47.9 \\
\%\end{array}$ & $\begin{array}{l}45.8 \\
\%\end{array}$ & $\begin{array}{l}50.2 \\
\%\end{array}$ & $\begin{array}{l}44.4 \\
\%\end{array}$ & $\begin{array}{l}3.1 \\
\%\end{array}$ & $\begin{array}{l}92.6 \\
\%\end{array}$ & $\begin{array}{l}51.7 \\
\%\end{array}$ & $4.0 \%$ & $\begin{array}{l}82.4 \\
\%\end{array}$ & $\begin{array}{l}43.4 \\
\%\end{array}$ \\
\hline Dagat & $17.3 \%$ & $\begin{array}{l}23.2 \\
\%\end{array}$ & $\begin{array}{l}25.9 \\
\%\end{array}$ & $\begin{array}{l}43.5 \\
\%\end{array}$ & $\begin{array}{l}23.8 \\
\%\end{array}$ & $\begin{array}{l}16.5 \\
\%\end{array}$ & $8.1 \%$ & $6.2 \%$ & $\begin{array}{l}48.1 \\
\%\end{array}$ & $\begin{array}{l}30.2 \\
\%\end{array}$ & $\begin{array}{l}69.2 \\
\%\end{array}$ & $\begin{array}{l}55.2 \\
\%\end{array}$ & $\begin{array}{l}4.2 \\
\%\end{array}$ & $\begin{array}{l}96.0 \\
\%\end{array}$ & $\begin{array}{l}38.6 \\
\%\end{array}$ & $5.4 \%$ & $\begin{array}{l}69.0 \\
\% \\
713\end{array}$ & $\begin{array}{l}46.7 \\
\%\end{array}$ \\
\hline Gasa & $10.9 \%$ & $\begin{array}{l}28.6 \\
\%\end{array}$ & $\begin{array}{l}20.2 \\
\%\end{array}$ & $\begin{array}{l}54.8 \\
\%\end{array}$ & $\begin{array}{l}18.3 \\
\%\end{array}$ & $\begin{array}{l}15.6 \\
\%\end{array}$ & $3.0 \%$ & $\begin{array}{l}10.0 \\
\%\end{array}$ & $\begin{array}{l}64.5 \\
\%\end{array}$ & $\begin{array}{l}24.7 \\
\%\end{array}$ & $\begin{array}{l}67.6 \\
\%\end{array}$ & $\begin{array}{l}59.1 \\
\%\end{array}$ & $\begin{array}{l}3.7 \\
\%\end{array}$ & $\begin{array}{l}90.6 \\
\%\end{array}$ & $\begin{array}{l}51.0 \\
\%\end{array}$ & $0.7 \%$ & $\begin{array}{l}71.3 \\
\%\end{array}$ & $\begin{array}{l}34.3 \\
\%\end{array}$ \\
\hline Haa & $8.7 \%$ & $\begin{array}{l}28.4 \\
\%\end{array}$ & $\begin{array}{l}25.9 \\
\%\end{array}$ & $\begin{array}{l}45.4 \\
\%\end{array}$ & $\begin{array}{l}13.1 \\
\%\end{array}$ & $\begin{array}{l}12.9 \\
\%\end{array}$ & $7.1 \%$ & $\begin{array}{l}11.6 \\
\%\end{array}$ & $\begin{array}{l}54.5 \\
\%\end{array}$ & $\begin{array}{l}32.9 \\
\%\end{array}$ & $\begin{array}{l}66.9 \\
\%\end{array}$ & $\begin{array}{l}56.0 \\
\%\end{array}$ & $\begin{array}{l}1.4 \\
\%\end{array}$ & $\begin{array}{l}90.4 \\
\%\end{array}$ & $\begin{array}{l}58.5 \\
\%\end{array}$ & $2.5 \%$ & $\begin{array}{l}73.0 \\
\%\end{array}$ & $\begin{array}{l}45.5 \\
\%\end{array}$ \\
\hline Lhuntse & $23.2 \%$ & $\begin{array}{l}55.3 \\
\%\end{array}$ & $\begin{array}{l}44.0 \\
\%\end{array}$ & $\begin{array}{l}39.6 \\
\%\end{array}$ & $\begin{array}{l}30.0 \\
\%\end{array}$ & $\begin{array}{l}27.7 \\
\%\end{array}$ & $\begin{array}{l}16.6 \\
\%\end{array}$ & $\begin{array}{l}17.6 \\
\%\end{array}$ & $\begin{array}{l}62.2 \\
\%\end{array}$ & $\begin{array}{l}16.3 \\
\%\end{array}$ & $\begin{array}{l}78.9 \\
\%\end{array}$ & $\begin{array}{l}62.0 \\
\%\end{array}$ & $\begin{array}{l}2.5 \\
\%\end{array}$ & $\begin{array}{l}89.6 \\
\%\end{array}$ & $\begin{array}{l}27.4 \\
\%\end{array}$ & $1.5 \%$ & $\begin{array}{l}58.8 \\
\%\end{array}$ & $\begin{array}{l}31.0 \\
\%\end{array}$ \\
\hline Mongar & $16.0 \%$ & $\begin{array}{l}55.3 \\
\%\end{array}$ & $\begin{array}{l}27.6 \\
\%\end{array}$ & $\begin{array}{l}49.1 \\
\%\end{array}$ & $\begin{array}{l}21.8 \\
\%\end{array}$ & $\begin{array}{l}23.3 \\
\%\end{array}$ & $\begin{array}{l}10.1 \\
\%\end{array}$ & $\begin{array}{l}10.5 \\
\%\end{array}$ & $\begin{array}{l}70.7 \\
\%\end{array}$ & $\begin{array}{l}33.2 \\
\%\end{array}$ & $\begin{array}{l}73.3 \\
\%\end{array}$ & $\begin{array}{l}61.6 \\
\%\end{array}$ & $\begin{array}{l}0.8 \\
\%\end{array}$ & $\begin{array}{l}92.4 \\
\%\end{array}$ & $\begin{array}{l}25.0 \\
\%\end{array}$ & $0.9 \%$ & $\begin{array}{l}55.7 \\
\%\end{array}$ & $\begin{array}{l}22.1 \\
\%\end{array}$ \\
\hline Paro & $.7 \%$ & $\begin{array}{l}44.7 \\
\%\end{array}$ & $\begin{array}{l}45.7 \\
\%\end{array}$ & $\begin{array}{l}42.9 \\
\%\end{array}$ & $\begin{array}{l}20.4 \\
\%\end{array}$ & $\begin{array}{l}21.5 \\
\%\end{array}$ & $\begin{array}{l}13.5 \\
\%\end{array}$ & $\begin{array}{l}10.0 \\
\%\end{array}$ & $\begin{array}{l}40.0 \\
\%\end{array}$ & $\begin{array}{l}34.7 \\
\%\end{array}$ & $\begin{array}{l}55.9 \\
\%\end{array}$ & $\begin{array}{l}48.2 \\
\%\end{array}$ & $\begin{array}{l}4.7 \\
\%\end{array}$ & $\begin{array}{l}92.1 \\
\%\end{array}$ & $\begin{array}{l}54.8 \\
\%\end{array}$ & $2.7 \%$ & $\begin{array}{l}60.2 \\
\%\end{array}$ & $\begin{array}{l}38.9 \\
\%\end{array}$ \\
\hline Pemagatshel & $22.9 \%$ & 56.0 & 35.3 & 56.8 & 31.0 & 30.2 & 16.8 & 21.4 & 61.7 & 12.9 & 76.9 & 59.1 & 1.9 & 97.8 & 15.5 & $1.2 \%$ & 47.5 & 25.0 \\
\hline
\end{tabular}


Karma Ura, Sabina Alkire, Tshoki Zangmo \& Karma Wangdi

\begin{tabular}{|c|c|c|c|c|c|c|c|c|c|c|c|c|c|c|c|c|c|c|}
\hline \multirow{3}{*}{ Punakha } & \multirow{3}{*}{$16.5 \%$} & $\%$ & $\%$ & $\%$ & $\%$ & $\%$ & $\%$ & $\%$ & $\%$ & $\%$ & $\%$ & $\%$ & $\%$ & $\%$ & \multirow{2}{*}{\multicolumn{2}{|c|}{$\begin{array}{l}\% \\
58.9\end{array}$}} & \multirow{3}{*}{$\begin{array}{l}\% \\
71.9 \\
\%\end{array}$} & \multirow{3}{*}{$\begin{array}{l}\% \\
47.4 \\
\%\end{array}$} \\
\hline & & 29.8 & 41.8 & 46.5 & 25.1 & 18.4 & 10.8 & 16.0 & 35.9 & 32.4 & 65.5 & 52.9 & 1.1 & 87.6 & & & & \\
\hline & & $\%$ & $\%$ & $\%$ & $\%$ & $\%$ & $\%$ & $\%$ & $\%$ & $\%$ & $\%$ & $\%$ & $\%$ & $\%$ & $\%$ & $1.2 \%$ & & \\
\hline Samdrup & & 54.2 & 53.1 & 50.0 & 28.3 & 38.4 & 15.0 & 20.9 & 66.5 & 25.6 & 71.1 & 56.5 & 3.5 & 94.6 & 21.8 & & 54.1 & 47.6 \\
\hline Jongkhar & $31.2 \%$ & $\%$ & $\%$ & $\%$ & $\%$ & $\%$ & $\%$ & $\%$ & $\%$ & $\%$ & $\%$ & $\%$ & $\%$ & $\%$ & $\%$ & $4.8 \%$ & $\%$ & $\%$ \\
\hline \multirow{2}{*}{ Samtse } & & 42.4 & 22.8 & 52.1 & 30.5 & 21.8 & & & 54.5 & 38.9 & 65.9 & 50.7 & 2.0 & 94.8 & 46.6 & & 84.0 & 36.3 \\
\hline & $11.7 \%$ & $\%$ & $\%$ & $\%$ & $\%$ & $\%$ & $9.1 \%$ & $8.5 \%$ & $\%$ & $\%$ & $\%$ & $\%$ & $\%$ & $\%$ & $\%$ & $8.3 \%$ & $\%$ & $\%$ \\
\hline \multirow{2}{*}{ Sarpang } & & 31.1 & 28.6 & 41.9 & 44.3 & 20.8 & & 12.9 & 56.1 & 53.6 & 55.2 & 49.1 & 0.8 & 93.2 & 34.8 & 13.1 & 62.0 & 37.4 \\
\hline & $18.5 \%$ & $\%$ & $\%$ & $\%$ & $\%$ & $\%$ & $8.4 \%$ & $\%$ & $\%$ & $\%$ & $\%$ & $\%$ & $\%$ & $\%$ & $\%$ & $\%$ & $\%$ & $\%$ \\
\hline \multirow{2}{*}{ Tashiyangtse } & & 36.4 & 47.3 & 35.3 & 29.7 & 33.4 & 19.1 & 19.1 & 77.6 & 22.6 & 77.3 & 63.5 & 3.6 & 93.8 & 21.9 & & 53.7 & 24.0 \\
\hline & $21.2 \%$ & $\%$ & $\%$ & $\%$ & $\%$ & $\%$ & $\%$ & $\%$ & $\%$ & $\%$ & $\%$ & $\%$ & $\%$ & $\%$ & $\%$ & $3.1 \%$ & $\%$ & $\%$ \\
\hline \multirow{2}{*}{ Tashigang } & & 38.6 & 48.4 & 41.2 & 29.5 & 36.4 & 12.2 & 21.4 & 66.3 & 21.7 & 83.2 & 65.6 & 2.1 & 95.2 & 13.4 & & 44.2 & 30.1 \\
\hline & $20.1 \%$ & $\%$ & $\%$ & $\%$ & $\%$ & $\%$ & $\%$ & $\%$ & $\%$ & $\%$ & $\%$ & $\%$ & $\%$ & $\%$ & $\%$ & $3.4 \%$ & $\%$ & $\%$ \\
\hline \multirow{2}{*}{ Thimphu } & & 43.7 & 39.9 & 50.4 & 15.8 & 14.6 & & & 43.6 & 38.9 & 33.8 & 28.4 & 5.3 & 86.6 & 41.9 & & 74.3 & 52.1 \\
\hline & $14.2 \%$ & $\%$ & $\%$ & $\%$ & $\%$ & $\%$ & $8.0 \%$ & $7.5 \%$ & $\%$ & $\%$ & $\%$ & $\%$ & $\%$ & $\%$ & $\%$ & $8.3 \%$ & $\%$ & $\%$ \\
\hline \multirow{2}{*}{ Trongsa } & & 38.0 & 42.4 & 39.4 & 22.4 & 29.4 & & 17.5 & 60.1 & 30.0 & 69.0 & 59.1 & 2.7 & 90.5 & 42.4 & & 62.1 & 57.4 \\
\hline & $20.3 \%$ & $\%$ & $\%$ & $\%$ & $\%$ & $\%$ & $8.7 \%$ & $\%$ & $\%$ & $\%$ & $\%$ & $\%$ & $\%$ & $\%$ & $\%$ & $3.7 \%$ & $\%$ & $\%$ \\
\hline \multirow{2}{*}{ Tsirang } & & 30.3 & 29.0 & 46.4 & 34.9 & 21.2 & 11.8 & 14.6 & 58.7 & 31.2 & 58.8 & 58.4 & 0.6 & 95.7 & 39.1 & & 67.9 & 36.5 \\
\hline & $19.4 \%$ & $\%$ & $\%$ & $\%$ & $\%$ & $\%$ & $\%$ & $\%$ & $\%$ & $\%$ & $\%$ & $\%$ & $\%$ & $\%$ & $\%$ & $5.8 \%$ & $\%$ & $\%$ \\
\hline Wangdue & & 35.8 & 29.6 & 48.0 & 19.8 & 22.1 & & 17.9 & 53.6 & 23.9 & 68.9 & 58.0 & 1.7 & 92.9 & 54.0 & & 73.8 & 44.3 \\
\hline Phodrang & $12.2 \%$ & $\%$ & $\%$ & $\%$ & $\%$ & $\%$ & $9.2 \%$ & $\%$ & $\%$ & $\%$ & $\%$ & $\%$ & $\%$ & $\%$ & $\%$ & $3.7 \%$ & $\%$ & $\%$ \\
\hline \multirow{2}{*}{ Zhemgang } & & 34.0 & 33.7 & 51.4 & 30.8 & 20.9 & & 10.0 & 46.3 & 25.8 & 66.8 & 57.7 & 1.0 & 92.7 & 28.2 & & 64.9 & 35.6 \\
\hline & $16.4 \%$ & $\%$ & $\%$ & $\%$ & $\%$ & $\%$ & $7.9 \%$ & $\%$ & $\%$ & $\%$ & $\%$ & $\%$ & $\%$ & $\%$ & $\%$ & $3.2 \%$ & $\%$ & $\%$ \\
\hline \multicolumn{19}{|l|}{ Region } \\
\hline & & 41.2 & 34.3 & 45.9 & 28.9 & 25.9 & 11.9 & 15.6 & 58.8 & 29.7 & 72.8 & 60.0 & 1.7 & 94.5 & 36.9 & & 63.0 & 34.0 \\
\hline \multirow[t]{2}{*}{ Rural } & $18.3 \%$ & $\%$ & $\%$ & $\%$ & $\%$ & $\%$ & $\%$ & $\%$ & $\%$ & $\%$ & $\%$ & $\%$ & $\%$ & $\%$ & $\%$ & $4.3 \%$ & $\%$ & $\%$ \\
\hline & & 41.2 & 38.0 & 50.7 & 19.0 & 17.2 & & & 44.7 & 42.7 & 35.1 & 29.4 & 5.4 & 87.4 & 40.4 & & 76.8 & 55.4 \\
\hline \multirow{2}{*}{$\begin{array}{l}\text { Urban } \\
\text { Gender }\end{array}$} & $13.1 \%$ & $\%$ & $\%$ & $\%$ & $\%$ & $\%$ & $6.8 \%$ & $9.0 \%$ & $\%$ & $\%$ & $\%$ & $\%$ & $\%$ & $\%$ & $\%$ & $6.8 \%$ & $\%$ & $\%$ \\
\hline & & 38.9 & 29.0 & 42.2 & 22.8 & 19.8 & & 10.2 & 50.1 & 34.7 & 56.0 & 40.7 & 3.2 & 89.2 & 38.3 & & 64.3 & 41.4 \\
\hline Male & $13.4 \%$ & $\%$ & $\%$ & $\%$ & $\%$ & $\%$ & $9.8 \%$ & $\%$ & $\%$ & $\%$ & $\%$ & $\%$ & $\%$ & $\%$ & $\%$ & $5.1 \%$ & $\%$ & $\%$ \\
\hline
\end{tabular}


An Extensive Analysis of GNH Index

\begin{tabular}{|c|c|c|c|c|c|c|c|c|c|c|c|c|c|c|c|c|c|c|}
\hline & & 43.5 & 41.6 & 52.2 & 29.7 & 27.3 & 11.2 & 17.4 & 59.9 & 31.8 & 69.2 & 62.7 & 2.3 & 95.9 & 37.4 & & 69.3 & 38.2 \\
\hline \multicolumn{19}{|l|}{$\begin{array}{l}\text { Female } \\
\text { Age group }\end{array}$} \\
\hline & & 51.9 & 47.9 & 59.1 & 19.7 & 18.6 & & 11.4 & 31.7 & 31.7 & 27.0 & 19.5 & 5.9 & 92.5 & 45.8 & 10.6 & 81.4 & 41.5 \\
\hline \multirow{2}{*}{$<=20$} & $11.2 \%$ & $\%$ & $\%$ & $\%$ & $\%$ & $\%$ & $3.4 \%$ & $\%$ & $\%$ & $\%$ & $\%$ & $\%$ & $\%$ & $\%$ & $\%$ & $\%$ & $\%$ & $\%$ \\
\hline & & 43.0 & 44.3 & 61.0 & 16.9 & 19.9 & & & 48.9 & 30.0 & 38.4 & 27.6 & 4.9 & 89.4 & 35.0 & & 72.3 & 48.3 \\
\hline \multirow[t]{2}{*}{$21-25$} & $12.5 \%$ & $\%$ & $\%$ & $\%$ & $\%$ & $\%$ & $3.0 \%$ & $9.4 \%$ & $\%$ & $\%$ & $\%$ & $\%$ & $\%$ & $\%$ & $\%$ & $6.2 \%$ & $\%$ & $\%$ \\
\hline & & 40.4 & 38.2 & 56.6 & 19.5 & 17.8 & & & 51.9 & 30.6 & 43.1 & 37.2 & 3.4 & 90.3 & 40.0 & & 69.9 & 48.8 \\
\hline \multirow[t]{2}{*}{$26-30$} & $11.3 \%$ & $\%$ & $\%$ & $\%$ & $\%$ & $\%$ & $5.9 \%$ & $7.8 \%$ & $\%$ & $\%$ & $\%$ & $\%$ & $\%$ & $\%$ & $\%$ & $7.0 \%$ & $\%$ & $\%$ \\
\hline & & 44.4 & 30.5 & 52.1 & 19.0 & 23.6 & & 10.1 & 59.6 & 36.4 & 56.4 & 46.3 & 2.6 & 92.6 & 33.5 & & 67.6 & 43.7 \\
\hline \multirow{2}{*}{$31-35$} & $13.4 \%$ & $\%$ & $\%$ & $\%$ & $\%$ & $\%$ & $6.8 \%$ & $\%$ & $\%$ & $\%$ & $\%$ & $\%$ & $\%$ & $\%$ & $\%$ & $3.0 \%$ & $\%$ & $\%$ \\
\hline & & 39.0 & 36.2 & 46.8 & 24.7 & 22.5 & & 13.3 & 59.5 & 35.3 & 62.6 & 53.6 & 3.5 & 91.1 & 36.5 & & 67.5 & 39.0 \\
\hline \multirow[t]{2}{*}{$36-40$} & $15.9 \%$ & $\%$ & $\%$ & $\%$ & $\%$ & $\%$ & $9.9 \%$ & $\%$ & $\%$ & $\%$ & $\%$ & $\%$ & $\%$ & $\%$ & $\%$ & $5.5 \%$ & $\%$ & $\%$ \\
\hline & & 41.6 & 29.2 & 45.9 & 21.7 & 20.9 & & & 65.1 & 38.5 & 72.4 & 61.7 & 1.7 & 90.1 & 40.8 & & 61.1 & 35.9 \\
\hline \multirow{2}{*}{$41-45$} & $16.5 \%$ & $\%$ & $\%$ & $\%$ & $\%$ & $\%$ & $9.0 \%$ & $9.3 \%$ & $\%$ & $\%$ & $\%$ & $\%$ & $\%$ & $\%$ & $\%$ & $4.4 \%$ & $\%$ & $\%$ \\
\hline & & 41.0 & 34.0 & 41.1 & 29.9 & 21.0 & 11.9 & 16.0 & 63.2 & 33.4 & 79.2 & 65.7 & 2.5 & 94.8 & 32.6 & & 64.6 & 32.6 \\
\hline \multirow[t]{2}{*}{$46-50$} & $18.9 \%$ & $\%$ & $\%$ & $\%$ & $\%$ & $\%$ & $\%$ & $\%$ & $\%$ & $\%$ & $\%$ & $\%$ & $\%$ & $\%$ & $\%$ & $2.5 \%$ & $\%$ & $\%$ \\
\hline & & 42.3 & 30.7 & 34.9 & 35.0 & 30.6 & 19.1 & 18.8 & 60.9 & 37.7 & 80.0 & 67.6 & 1.2 & 94.2 & 38.2 & & 60.7 & 35.8 \\
\hline \multirow[t]{2}{*}{$51-55$} & $20.7 \%$ & $\%$ & $\%$ & $\%$ & $\%$ & $\%$ & $\%$ & $\%$ & $\%$ & $\%$ & $\%$ & $\%$ & $\%$ & $\%$ & $\%$ & $5.4 \%$ & $\%$ & $\%$ \\
\hline & & 39.7 & 31.0 & 34.3 & 36.1 & 27.1 & 19.5 & 18.8 & 58.8 & 34.1 & 81.5 & 67.4 & 0.7 & 96.6 & 36.6 & & 63.6 & 29.8 \\
\hline \multirow[t]{2}{*}{$56-60$} & $20.7 \%$ & $\%$ & $\%$ & $\%$ & $\%$ & $\%$ & $\%$ & $\%$ & $\%$ & $\%$ & $\%$ & $\%$ & $\%$ & $\%$ & $\%$ & $4.1 \%$ & $\%$ & $\%$ \\
\hline & & 32.1 & 32.1 & 31.8 & 38.5 & 39.7 & 18.8 & 22.9 & 51.1 & 27.0 & 93.4 & 75.3 & 0.2 & 96.9 & 40.6 & & 53.3 & 31.4 \\
\hline \multirow[t]{2}{*}{ 61-65 } & $23.9 \%$ & $\%$ & $\%$ & $\%$ & $\%$ & $\%$ & $\%$ & $\%$ & $\%$ & $\%$ & $\%$ & $\%$ & $\%$ & $\%$ & $\%$ & $2.0 \%$ & $\%$ & $\%$ \\
\hline & & 35.9 & 30.1 & 29.8 & 52.6 & 35.8 & 25.1 & 32.1 & 41.4 & 26.5 & 96.3 & 78.5 & 0.6 & 97.3 & 44.2 & & 63.7 & 32.9 \\
\hline$>65$ & $36.2 \%$ & $\%$ & $\%$ & $\%$ & $\%$ & $\%$ & $\%$ & $\%$ & $\%$ & $\%$ & $\%$ & $\%$ & $\%$ & $\%$ & $\%$ & $2.0 \%$ & $\%$ & $\%$ \\
\hline
\end{tabular}


Table VII (cont.) Increasing Happiness - Raw headcounts: The table shows the proportion of people who experience sufficiency in each indicator. Suggested citation: Ura, Karma; Alkire, Sabina; and Zangmo, Tshoki (Nov 2011) www.grossnationalhappiness.com ophi.qeh.ox.ac.uk

\begin{tabular}{|c|c|c|c|c|c|c|c|c|c|c|c|c|c|c|c|}
\hline & \multicolumn{3}{|c|}{ Good governance } & \multicolumn{5}{|c|}{ Community vitality } & \multicolumn{4}{|c|}{ Ecological diversity and resilience } & \multicolumn{3}{|c|}{ Living standards } \\
\hline & 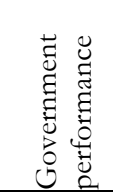 & 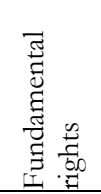 & 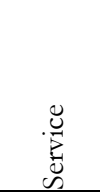 & 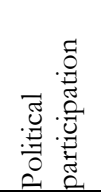 & 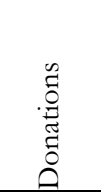 & 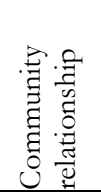 & 兺 & 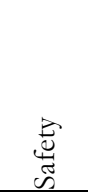 & 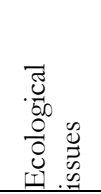 & 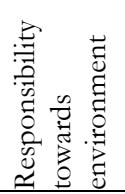 & 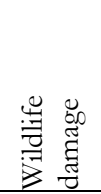 & 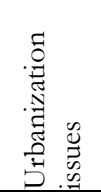 & 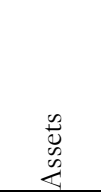 & 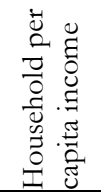 & 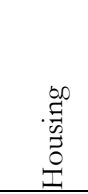 \\
\hline & \multicolumn{15}{|c|}{ Raw headcount } \\
\hline Bhutan & $20.5 \%$ & $37.8 \%$ & $59.1 \%$ & $3.4 \%$ & $3.7 \%$ & $37.5 \%$ & $7.1 \%$ & $3.8 \%$ & $0.8 \%$ & $5.7 \%$ & $42.7 \%$ & $15.2 \%$ & $25.8 \%$ & $46.6 \%$ & $53.7 \%$ \\
\hline Dagan & $.8 \%$ & $34.7 \%$ & $69.5 \%$ & $7 \%$ & $.9 \%$ & $32.3 \%$ & $6.1 \%$ & $2.4 \%$ & $20.2 \%$ & $39.2 \%$ & $51.0 \%$ & $0.3 \%$ & $20.0 \%$ & $45.2 \%$ & $57.4 \%$ \\
\hline Gasa & $19.9 \%$ & $39.4 \%$ & $69.2 \%$ & $28.5 \%$ & $54.9 \%$ & $22.6 \%$ & $5.7 \%$ & $1.8 \%$ & $40.4 \%$ & $11.0 \%$ & $38.4 \%$ & $3.1 \%$ & $22.0 \%$ & $26.8 \%$ & $82.2 \%$ \\
\hline $\mathrm{Haa}$ & $22.6 \%$ & $33.5 \%$ & $47.8 \%$ & $42.9 \%$ & $53.9 \%$ & $36.6 \%$ & $5.6 \%$ & $4.8 \%$ & $32.8 \%$ & $12.5 \%$ & $49.7 \%$ & $3.0 \%$ & $28.9 \%$ & $26.7 \%$ & $49.3 \%$ \\
\hline Lhuntse & $20.9 \%$ & $53.1 \%$ & $70.7 \%$ & $34.8 \%$ & $45.9 \%$ & $22.8 \%$ & $10.6 \%$ & $1.3 \%$ & $26.1 \%$ & $13.9 \%$ & $57.2 \%$ & $0.3 \%$ & $31.8 \%$ & $77.7 \%$ & $84.4 \%$ \\
\hline & $1 \%$ & $38.0 \%$ & 60 & $32.7 \%$ & $\%$ & $24.1 \%$ & $8.2 \%$ & $4.4 \%$ & $21.1 \%$ & $13.5 \%$ & $1 \%$ & $6.4 \%$ & $\% \%$ & $60.7 \%$ & $76.8 \%$ \\
\hline Paro & $.6 \%$ & $34.8 \%$ & 50 & $\%$ & $55.6 \%$ & $40.0 \%$ & 5.6 & $5.5 \%$ & $14.2 \%$ & $16.5 \%$ & $20.8 \%$ & $11.8 \%$ & $\%$ & $28.0 \%$ & $36.2 \%$ \\
\hline Pema & $.0 \%$ & $59.1 \%$ & $78.9 \%$ & 2 & $40.4 \%$ & $29.8 \%$ & 8.2 & $3.7 \%$ & 4.0 & $19.6 \%$ & $71.4 \%$ & $1.5 \%$ & $46.1 \%$ & $55.7 \%$ & $67.1 \%$ \\
\hline Tas & $16.7 \%$ & $41.7 \%$ & $58.4 \%$ & $26.1 \%$ & $46.2 \%$ & $29.3 \%$ & $3.9 \%$ & $2.8 \%$ & $12.6 \%$ & $13.1 \%$ & $67.2 \%$ & $1.1 \%$ & $43.4 \%$ & $67.9 \%$ & $79.8 \%$ \\
\hline & $15.0 \%$ & $48.8 \%$ & $61.1 \%$ & $26.8 \%$ & $35.8 \%$ & $27.0 \%$ & $7.3 \%$ & $1.8 \%$ & $33.2 \%$ & $19.9 \%$ & $66.4 \%$ & $5.7 \%$ & $44.2 \%$ & $71.5 \%$ & $79.7 \%$ \\
\hline Thimphu & $26.1 \%$ & $38.4 \%$ & $29.6 \%$ & $87.6 \%$ & $76.5 \%$ & $65.1 \%$ & $7.5 \%$ & $7.3 \%$ & $38.2 \%$ & $15.4 \%$ & $3.8 \%$ & $64.3 \%$ & $26.1 \%$ & $14.2 \%$ & $22.7 \%$ \\
\hline Trongsa & $19.8 \%$ & $42.2 \%$ & $69.2 \%$ & $40.6 \%$ & $58.1 \%$ & $41.2 \%$ & $11.8 \%$ & $4.1 \%$ & $32.4 \%$ & $18.6 \%$ & $56.2 \%$ & $12.4 \%$ & $30.6 \%$ & $48.4 \%$ & $55.4 \%$ \\
\hline Tsirang & $14.6 \%$ & $19.2 \%$ & $78.5 \%$ & $19.9 \%$ & $44.9 \%$ & $18.2 \%$ & $6.4 \%$ & $3.5 \%$ & $27.1 \%$ & $8.6 \%$ & $62.5 \%$ & $2.7 \%$ & $13.6 \%$ & $51.9 \%$ & $52.9 \%$ \\
\hline
\end{tabular}


An Extensive Analysis of GNH Index

\begin{tabular}{|c|c|c|c|c|c|c|c|c|c|c|c|c|c|c|c|}
\hline Wangdue Phodrang & $20.5 \%$ & $37.0 \%$ & $70.9 \%$ & $33.2 \%$ & $50.9 \%$ & $38.8 \%$ & $8.2 \%$ & $3.0 \%$ & $45.0 \%$ & $14.9 \%$ & $49.1 \%$ & $19.1 \%$ & $16.1 \%$ & $44.8 \%$ & $60.3 \%$ \\
\hline \multicolumn{16}{|l|}{ Region } \\
\hline Rural & $18.7 \%$ & $37.5 \%$ & $70.0 \%$ & $27.8 \%$ & $45.7 \%$ & $27.9 \%$ & $7.1 \%$ & $3.3 \%$ & $26.8 \%$ & $15.8 \%$ & $58.2 \%$ & $2.1 \%$ & $27.2 \%$ & $59.2 \%$ & $66.4 \%$ \\
\hline Urban & $25.4 \%$ & $38.7 \%$ & $29.7 \%$ & $85.1 \%$ & $75.4 \%$ & $63.4 \%$ & $7.1 \%$ & $5.2 \%$ & $41.6 \%$ & $15.6 \%$ & $0.9 \%$ & $50.3 \%$ & $22.3 \%$ & $12.7 \%$ & $19.7 \%$ \\
\hline Male & $20.3 \%$ & $34.4 \%$ & $61.5 \%$ & $36.1 \%$ & $47.7 \%$ & $33.3 \%$ & $5.2 \%$ & $3.2 \%$ & $30.5 \%$ & $13.5 \%$ & $45.9 \%$ & $13.6 \%$ & $23.2 \%$ & $49.2 \%$ & $55.6 \%$ \\
\hline Female & $20.8 \%$ & $41.2 \%$ & $56.6 \%$ & $50.6 \%$ & $59.7 \%$ & $41.7 \%$ & $8.9 \%$ & $4.4 \%$ & $31.1 \%$ & $18.0 \%$ & $39.4 \%$ & $16.7 \%$ & $28.5 \%$ & $43.9 \%$ & $51.8 \%$ \\
\hline \multicolumn{16}{|l|}{ Age group } \\
\hline $31-35$ & $20.3 \%$ & $41.6 \%$ & $58.5 \%$ & $43.1 \%$ & $56.9 \%$ & $42.1 \%$ & $6.6 \%$ & $3.8 \%$ & $30.8 \%$ & $14.6 \%$ & $37.2 \%$ & $16.4 \%$ & $23.0 \%$ & $48.4 \%$ & $50.7 \%$ \\
\hline $36-40$ & $21.9 \%$ & $41.2 \%$ & $60.5 \%$ & $42.1 \%$ & $49.9 \%$ & $39.7 \%$ & $8.2 \%$ & $3.1 \%$ & $34.0 \%$ & $13.6 \%$ & $40.0 \%$ & $20.6 \%$ & $26.2 \%$ & $45.2 \%$ & $51.9 \%$ \\
\hline $41-45$ & $22.0 \%$ & $31.1 \%$ & $59.5 \%$ & $37.3 \%$ & $43.4 \%$ & $29.2 \%$ & $7.5 \%$ & $3.2 \%$ & $31.7 \%$ & $16.5 \%$ & $47.3 \%$ & $12.9 \%$ & $23.6 \%$ & $50.6 \%$ & $57.4 \%$ \\
\hline $46-50$ & $17.3 \%$ & $37.8 \%$ & $64.7 \%$ & $27.7 \%$ & $43.3 \%$ & $25.5 \%$ & $4.4 \%$ & $2.5 \%$ & $30.0 \%$ & $13.9 \%$ & $58.4 \%$ & $9.5 \%$ & $21.5 \%$ & $54.8 \%$ & $62.1 \%$ \\
\hline $51-55$ & $17.1 \%$ & $35.6 \%$ & $64.5 \%$ & $26.7 \%$ & $45.3 \%$ & $24.2 \%$ & $4.4 \%$ & $2.1 \%$ & $29.8 \%$ & $15.0 \%$ & $60.1 \%$ & $5.7 \%$ & $25.7 \%$ & $52.7 \%$ & $65.8 \%$ \\
\hline $56-60$ & $16.9 \%$ & $35.9 \%$ & $63.9 \%$ & $21.9 \%$ & $47.8 \%$ & $22.0 \%$ & $5.0 \%$ & $2.2 \%$ & $30.5 \%$ & $14.3 \%$ & $60.9 \%$ & $6.2 \%$ & $25.1 \%$ & $53.9 \%$ & $64.2 \%$ \\
\hline
\end{tabular}


Karma Ura, Sabina Alkire, Tshoki Zangmo \& Karma Wangdi

Table VIII GNH and other measures for Bhutan This table provides the at-a-glance information on the GNH Index and other income and Human Development indicators

Suggested citation: Ura, Karma; Alkire, Sabina; and Zangmo, Tshoki (Nov 2011) www.grossnationalhappiness.com ophi.qeh.ox.ac.uk

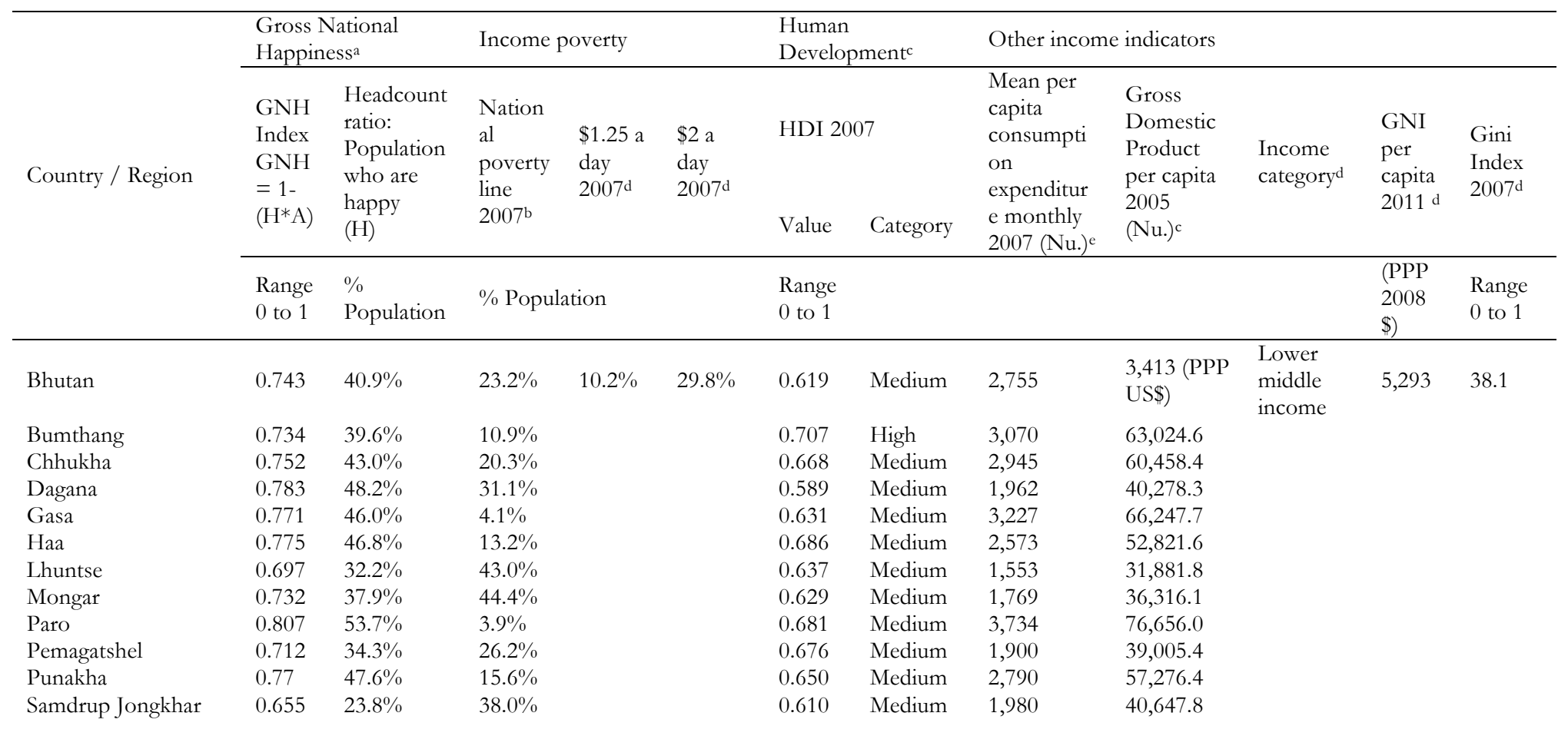


An Extensive Analysis of GNH Index

\begin{tabular}{llllllll} 
Samtse & 0.736 & $39.1 \%$ & $46.8 \%$ & 0.585 & Medium & 1,668 & $34,242.7$ \\
Sarpang & 0.795 & $51.3 \%$ & $19.4 \%$ & 0.626 & Medium & 2,181 & $44,774.1$ \\
Tashiyangtse & 0.698 & $31.4 \%$ & $14.3 \%$ & 0.616 & Medium & 2,302 & $47,258.2$ \\
Tashigang & 0.708 & $33.2 \%$ & $29.3 \%$ & 0.649 & Medium & 1,936 & $39,744.5$ \\
Thimphu & 0.773 & $46.4 \%$ & $2.4 \%$ & 0.727 & High & 5,346 & $109,749.0$ \\
Trongsa & 0.684 & $28.7 \%$ & $22.2 \%$ & 0.673 & Medium & 2,552 & $52,390.5$ \\
Tsirang & 0.77 & $47.7 \%$ & $13.9 \%$ & 0.658 & Medium & 2,570 & $52,760.0$ \\
Wangdue Phodrang & 0.738 & $40.1 \%$ & $15.8 \%$ & 0.656 & Medium & 2,709 & $55,613.6$ \\
Zhemgang & 0.753 & $43.4 \%$ & $52.9 \%$ & 0.651 & Medium & 1,738 & $35,679.7$ \\
\hline
\end{tabular}


Karma Ura, Sabina Alkire, Tshoki Zangmo \& Karma Wangdi

Table IX Happiness Gradient by Dzongkhag, Gender, and Age The table shows the proportion of people who belong to each sector of the happiness gradient.

Suggested citation: Ura, Karma; Alkire, Sabina; and Zangmo, Tshoki (Nov 2011) www.grossnationalhappiness.com ophi.qeh.ox.ac.uk

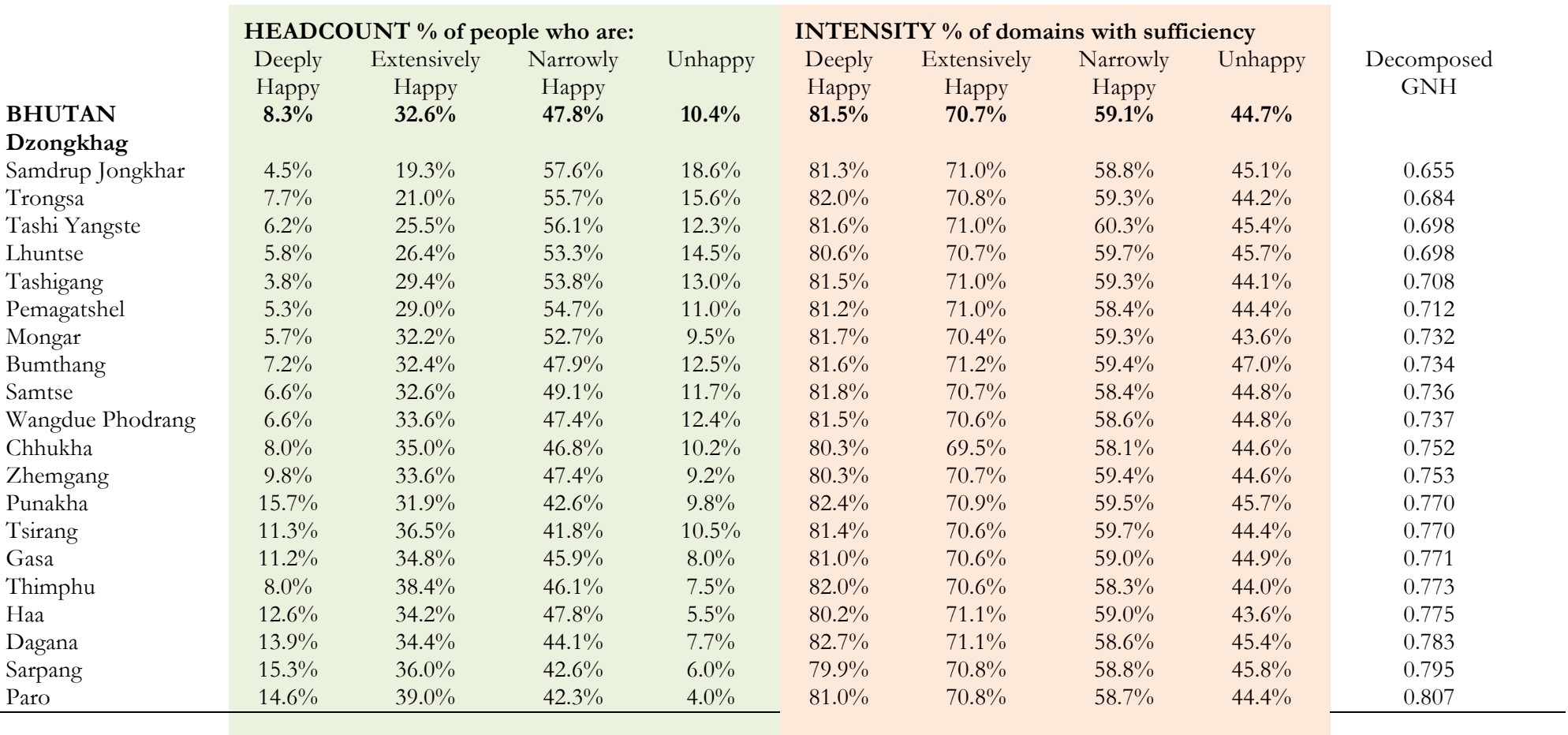


An Extensive Analysis of GNH Index

\begin{tabular}{|c|c|c|c|c|c|c|c|c|c|}
\hline Gender & $\begin{array}{l}\text { Deeply } \\
\text { Happv }\end{array}$ & $\begin{array}{c}\text { Extensively } \\
\text { Happy }\end{array}$ & $\begin{array}{c}\text { Narrowly } \\
\text { Happy }\end{array}$ & Unhappy & $\begin{array}{l}\text { Deeply } \\
\text { Happy }\end{array}$ & $\begin{array}{c}\text { Extensively } \\
\text { Happy }\end{array}$ & $\begin{array}{c}\text { Narrowly } \\
\text { Happy }\end{array}$ & Unhappy & $\begin{array}{c}\text { Decomposed } \\
\text { GNH }\end{array}$ \\
\hline Male & $11.1 \%$ & $37.4 \%$ & $45.0 \%$ & $6.5 \%$ & $81.8 \%$ & $70.8 \%$ & $59.6 \%$ & $44.8 \%$ & 0.783 \\
\hline Female & $5.4 \%$ & $27.7 \%$ & $52.5 \%$ & $14.3 \%$ & $80.8 \%$ & $70.6 \%$ & $58.7 \%$ & $44.6 \%$ & 0.704 \\
\hline & Deeply & Extensively & Narrowly & Unhappy & Deeply & Extensively & Narrowly & Unhappy & Decomposed \\
\hline Age & Happy & Happy & Happy & & Happy & Happy & Happy & & GNH \\
\hline$<=20$ & $8.7 \%$ & $33.7 \%$ & $52.5 \%$ & $5.1 \%$ & $80.5 \%$ & $70.8 \%$ & $59.4 \%$ & $45.0 \%$ & 0.759 \\
\hline $21-25$ & $9.0 \%$ & $40.7 \%$ & $42.8 \%$ & $7.5 \%$ & $81.5 \%$ & $70.6 \%$ & $59.3 \%$ & $45.4 \%$ & 0.785 \\
\hline $26-30$ & $9.3 \%$ & $38.0 \%$ & $46.3 \%$ & $6.4 \%$ & $81.1 \%$ & $71.0 \%$ & $59.8 \%$ & $44.5 \%$ & 0.778 \\
\hline $31-35$ & $7.1 \%$ & $35.5 \%$ & $48.0 \%$ & $9.4 \%$ & $81.9 \%$ & $70.5 \%$ & $59.6 \%$ & $45.3 \%$ & 0.754 \\
\hline $36-40$ & $9.5 \%$ & $28.6 \%$ & $52.0 \%$ & $9.9 \%$ & $82.1 \%$ & $70.7 \%$ & $59.2 \%$ & $42.9 \%$ & 0.731 \\
\hline $41-45$ & $8.9 \%$ & $30.2 \%$ & $48.4 \%$ & $12.5 \%$ & $82.2 \%$ & $70.7 \%$ & $59.6 \%$ & $45.2 \%$ & 0.736 \\
\hline $46-50$ & $8.2 \%$ & $32.8 \%$ & $47.8 \%$ & $11.2 \%$ & $80.7 \%$ & $70.6 \%$ & $58.5 \%$ & $45.2 \%$ & 0.740 \\
\hline $51-55$ & $8.0 \%$ & $26.0 \%$ & $54.3 \%$ & $11.7 \%$ & $81.5 \%$ & $70.7 \%$ & $58.4 \%$ & $44.7 \%$ & 0.710 \\
\hline $56-60$ & $8.5 \%$ & $29.1 \%$ & $50.6 \%$ & $11.9 \%$ & $81.3 \%$ & $71.2 \%$ & $58.5 \%$ & $44.7 \%$ & 0.725 \\
\hline $61-65$ & $8.3 \%$ & $23.9 \%$ & $50.0 \%$ & $17.9 \%$ & $81.1 \%$ & $70.7 \%$ & $58.7 \%$ & $45.7 \%$ & 0.696 \\
\hline$>65$ & $3.8 \%$ & $24.9 \%$ & $50.7 \%$ & $20.6 \%$ & $81.2 \%$ & $70.8 \%$ & $58.5 \%$ & $43.7 \%$ & 0.674 \\
\hline
\end{tabular}

